\title{
Das Grammatikalitätsphänomen in Husserls Phänomenologie
}

\author{
Dissertation \\ zur Erlangung des philosophischen Doktorgrades \\ an der Philosophischen Fakultät der Georg-August-Universität Göttingen
}

\author{
vorgelegt von \\ Jiahao $\mathrm{Wu}$ \\ aus Guangdong, VR China
}

Göttingen 2020 


\section{Inhalt}

EINLEITUNG. 6

\section{ABSCHNITT: DAS GRAMMATIKALITÄTSPHÄNOMEN DER BEDEUTUNGEN}

\section{KAPITEL VORBEREITENDE ANALYSEN}

$\S 1$. Husserls Konzeption von ,reiner Grammatik“............................................... 9

$\S 2$. Vorbereitende Analysen I: die Idealität der Bedeutungen.................................... 9

§3. Vorbereitende Analysen II: die Rede von Spezies...........................................11

$\S 4$. Vorbereitende Analysen III: intentionale Erlebnisse ....................................... 15

$\S 5$. Vorbereitende Analysen IV: Bedeutungsintention und Bedeutungserfüllung....... 20

§6. Vorbereitende Analysen V: Husserls Mereologie ............................................ 29

\section{KAPITEL REINE GRAMMATIK UND EMPIRISCHE GRAMMATIK}

§7. Kategorematische und synkategorematische Ausdrücke................................ 32

$\S 8$. Selbständige und unselbständige Bedeutung .................................................. 34

§. Intentionlitäts-Abhängigkeit/-Unabhängigkeit von Bedeutungen ...................... 37

$\S 10$. Bedeutungskategorien und die Gesetze der Bedeutungskomplikation............... 39

§11. Apriorische Grammatik vs. empirische Grammatik ....................................... 42

§12. Modifikationsgesetze von Bedeutungen I................................................ 44

§13. Modifikationsgesetze von Bedeutungen II ................................................ 46

$\S 14$. Zusammenfassung des Kapitels und das Grammatikalitätsphänomen der

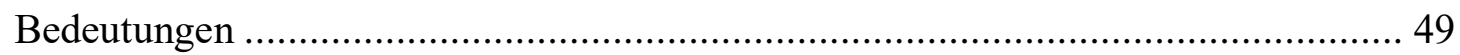

\section{KAPITEL REINE GRAMMATIK UND FORMALE LOGIK}

§15. Reine Grammatik im Rahmen der formalen Logik ...................................... 52

§16. Reine Formenlehre und reine Geltungslehre der Bedeutungen ......................... 52

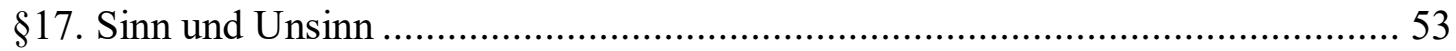

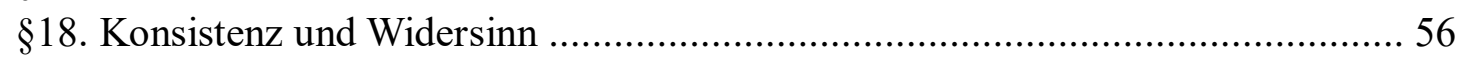

§19. Unterschied und Zusammenhang der beiden Bedeutungslehren ...................... 58

\$20. Die Dreischichtung der formalen Logik von Bedeutungen ............................. 60

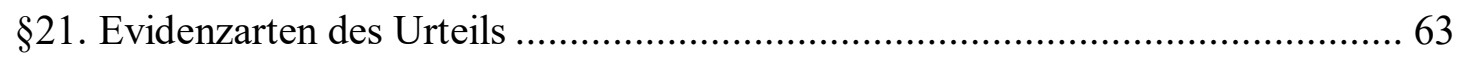

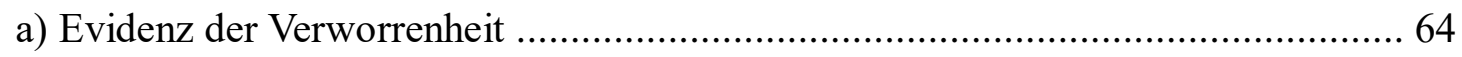

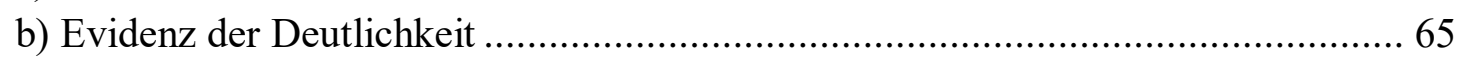

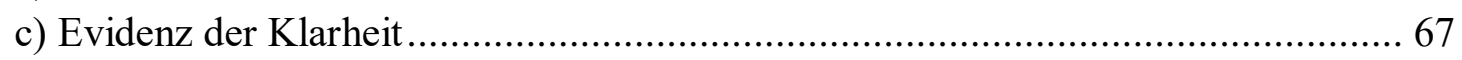

§22. Der Unterschied und Zusammenhang zwischen formaler Apophantik und

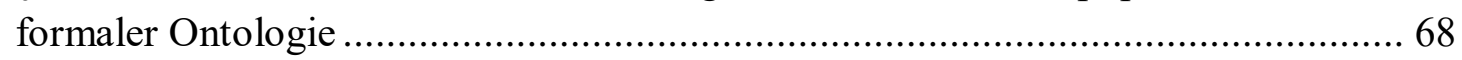


§23. Die subjektive Begründung für den Unterschied zwischen formaler Apophantik

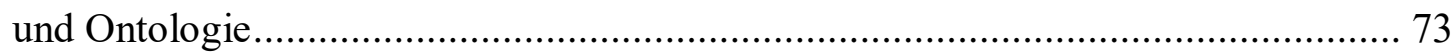

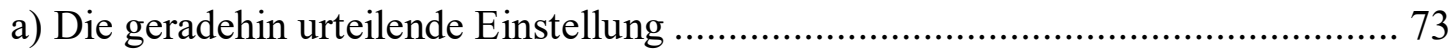

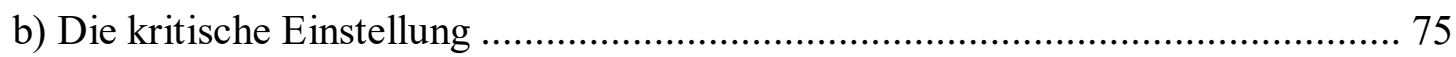

c) Zusammenfassung des Paragraphen.............................................................. 79

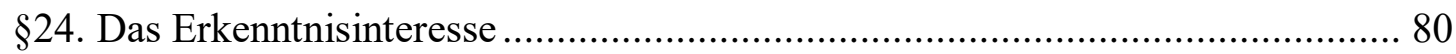

\$25. Das Erkenntnisinteresse und die Formenlehre .............................................. 82

$\S 26$. Zusammenfassung und Ausblick in den nächsten Abschnitt .......................... 83

\section{ABSCHNITT: DAS GRAMMATIKALITÄTSPHÄNOMEN IM RAHMEN DER TRANSZENDENTALEN PHÄNOMENOLOGIE}

\section{KAPITEL DIE PHÄNOMENOLOGISCHE REDUKTION}

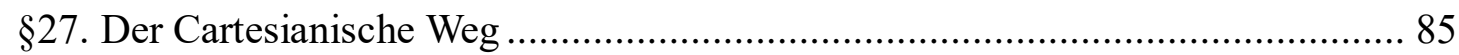

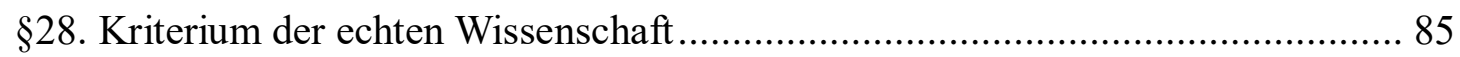

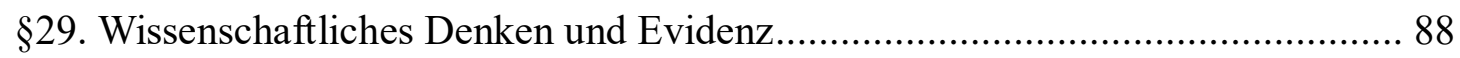

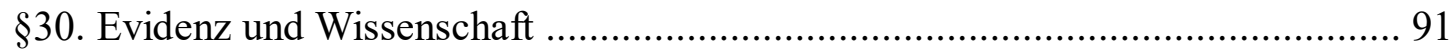

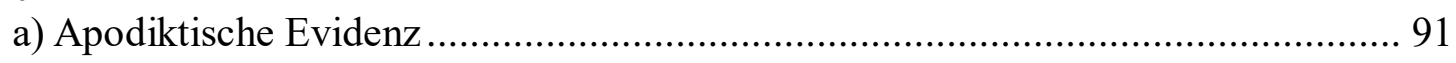

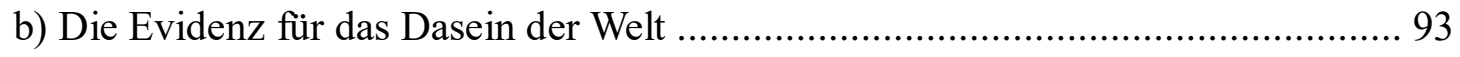

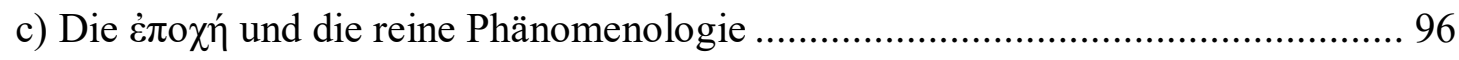

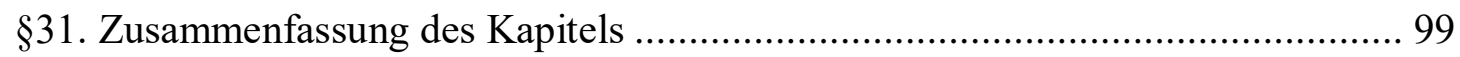

\section{KAPITEL DIE NOETISCH-NOEMATISCHEN ANALYSEN IM RAHMEN DER TRANSZENDENTALEN PHÄNOMENOLOGIE I}

§32. Intentionale und reelle Komponenten des intentionalen Erlebnisses ................100

a) Intentionale und reelle Inhalte in Logischen Untersuchungen ............................100

b) Das Noema als intentionaler Inhalt in Ideen I .................................................101

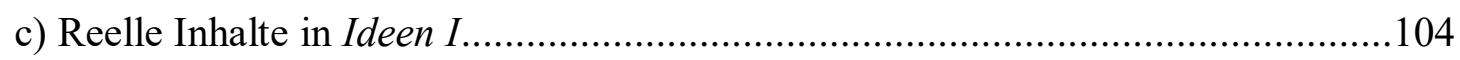

d) Einführung in die Konstitutionsanalysen und die Idee der transzendentalen

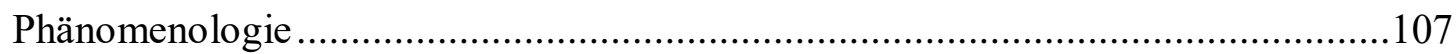

§33. Das Prinzip des noetisch-noematischen Parallelismus ................................... 112

$\S 34$. Noetische Sinngebung und noematischer Sinn ........................................... 114

$\S 35$. Noetischer Repräsentationscharakter und noematischer Anschauungscharakter

§36. Noetischer Aufmerksamkeitscharakter und noematischer Auffälligkeitscharakter

\$37. Noetischer Glaubenscharakter und noematischer Seinscharakter ....................121

$\S 37 *$. Exkurs: Setzungscharakter von nicht-objektivierenden Akten ......................126

§38. Setzende Sinngebung und noematischer Satz .............................................128

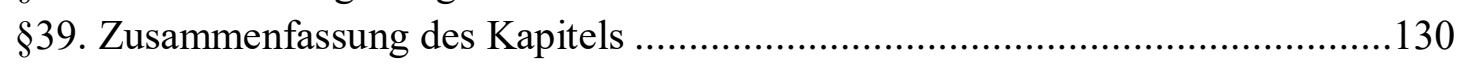




\section{KAPITEL DIE NOETISCH-NOEMATISCHEN ANALYSEN IM RAHMEN DER TRANSZENDENTALEN PHÄNOMENOLOGIE II}

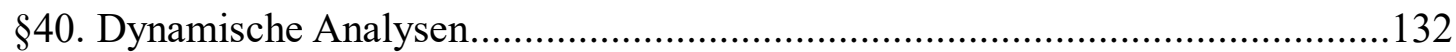

\$41. Die synthetische Einheit von Wahrnehmungsakten .......................................134

a) Das jeweilige Wahrnehmungsnoema als Erscheinung ....................................134

b) Enthüllung des Horizonts und der kontinuierliche Wahrnehmungsprozess ........137

$\S 42$. Das bestimmbare X und der Gegenstand der Wahrnehmung ..........................140

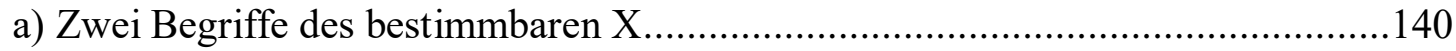

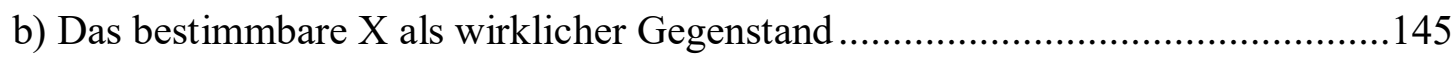

c) Existenzneutrale Spezifikation des bestimmbaren $X$.....................................147

§43. Urteilsnoema, Urteilsbedeutung, und das X in Urteilsakten............................150

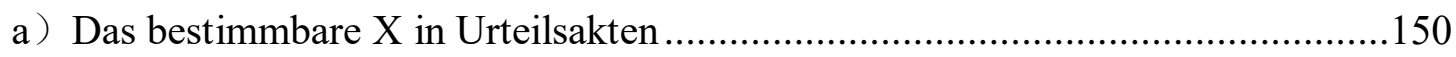

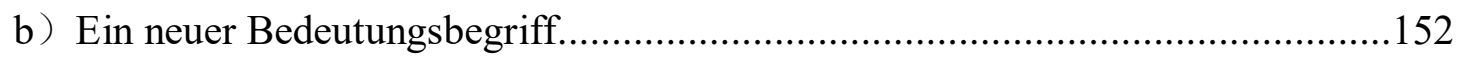

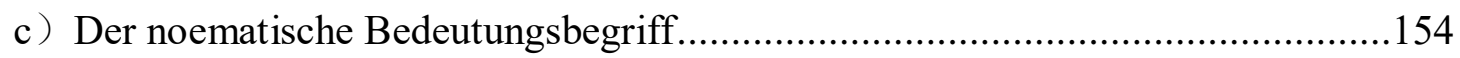

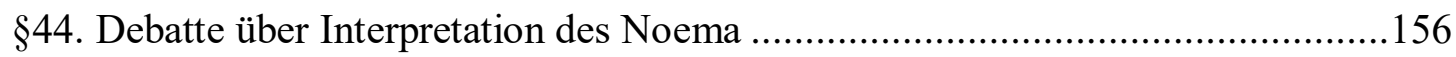

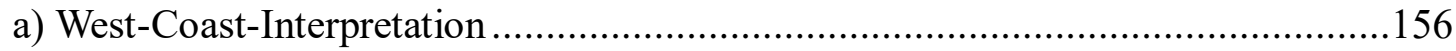

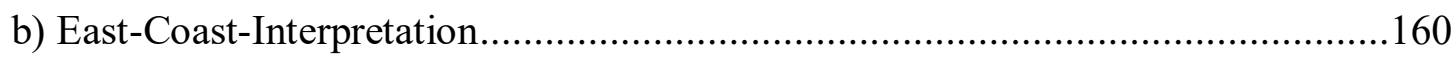

c) Quelle der Schwierigkeiten und ihre Überwindung ...........................................163

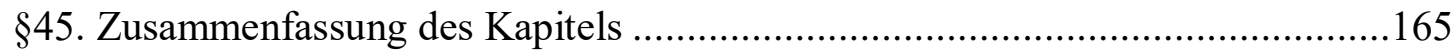

\section{KAPITEL REINTERPRETATION DER GRAMMATIKALITÄTSTHESEN IM RAHMEN DER TRANSZENDENTALEN PHÄNOMENOLOGIE}

$\S 46$. Formenlehre der noematischen Sätze/Sinne

$\S 47$. Reinterpretation der Grammatikalitätsthese der Bedeutungen im Rahmen der noetisch-noematischen Analysen

§48. Reinterpretation der Grammatikalitätsthese der Gegenstände im Rahmen der noetisch-noematischen Analysen

\$49. Der Zusammenhang der Grammatikalitätsthesen ..........................................173

§50. Zusammenfassung und Ausblick in den nächsten Abschnitt ...........................175

\section{ABSCHNITT: DAS GRAMMATIKALITÄTSPHÄNOMEN}

\section{DER BEDEUTUNGERFÜLLENDEN AKTE}

\section{KAPITEL EINLEITUNG IN DIE URSPRUNGSANALYSEN VON ERFAHRUNG UND URTEIL}

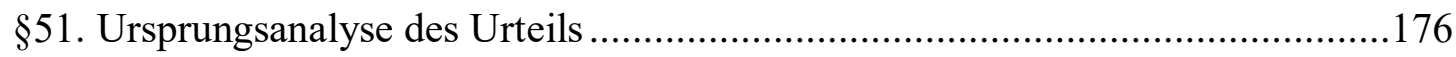

§52. Von der formalen zur transzendentalen Logik ...............................................176

$\S 53$. Von der transzendentalen Logik zur Konsitutionsanalyse des evidenten Urteils

$\S 54$. Von der Konstitutionsanalyse des evidenten Urteils zur Ursprungsanalyse des Urteils 


\section{KAPITEL SYNTAKTISCHE LEISTUNGEN DER ERFAHRUNG}

§57. Die Stufen des Erkenntnisstrebens

§58. Das passiv konstituierte Erfahrungsfeld und die affizierende Kraft auf das Subjekt

§59. Schlichte Erfassung und Explikation

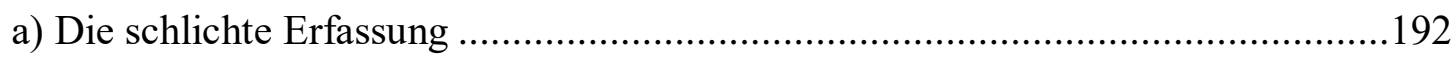

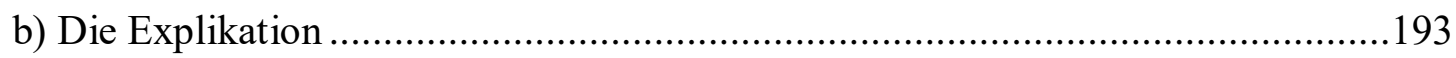

c) Die Sinnesbereicherung der Explikation und die dabei entstehenden Formen von

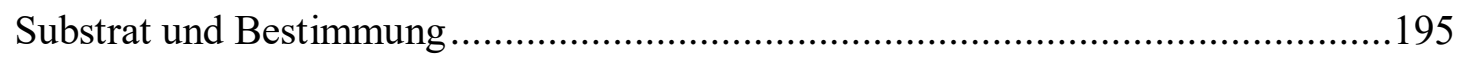

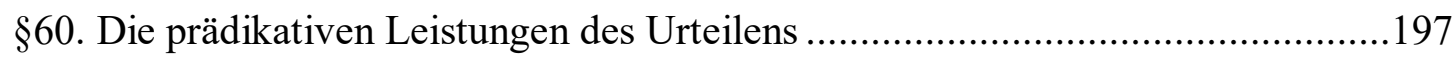

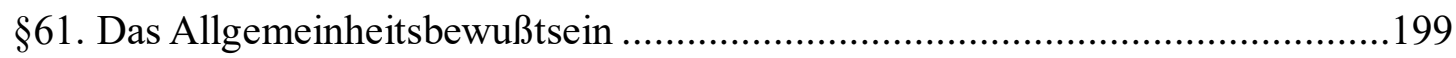

§62. Ursprungsanalysen für kompliziertere Urteilsformen ..................................202

a) Ursprungsanalysen für die Urteilsform , $\mathrm{S}$ ist $\mathrm{p}$ und $\mathrm{q}$ und $\mathrm{r}$... “.......................202

b) Ursprungsanalysen für Attribution enthaltende Urteilsformen............................203

c) Der Unterschied zwischen ,Ist“-Urteil und ,Hat"-Urteil .................................205

d) Ursprungsanalysen für beziehende Urteilsformen ........................................206

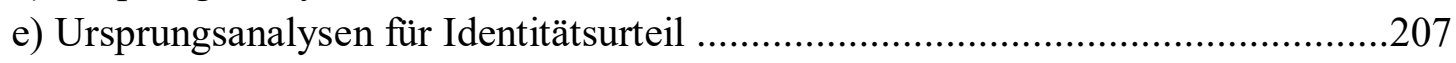

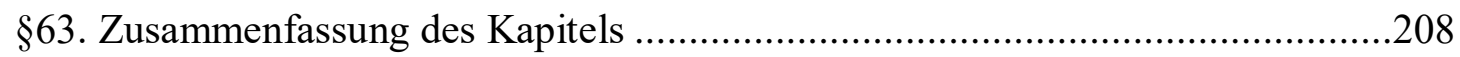

\section{KAPITEL GRAMMATIKALITÄTSTHESEN IM BEREICH DER BEDEUTUNGERFÜLLENDEN AKTE}

$\S 64$. Vor-syntaktische Formen vs. vor-grammatische Formen

$\S 65$. Das (Vor-)Grammatikalitätsphänomen der noematischen Sätze/Sinne von bedeutungerfüllenden Akten .....

§66. Das (Vor-)Grammatikalitätsphänomen des bestimmbaren X von bedeutungerfüllenden Akten....

$\S 67$. Das noetische (Vor-)Grammatikalitätsphänomen von bedeutungerfüllenden

SCHLUSSWORT 


\section{EINLEITUNG}

Das im Titel der vorliegenden Arbeit angekündigte Thema - das Grammatikalitätsphänomen - ist ein Begriff, den diese Untersuchung, ausgehend von Husserls Logischen Untersuchungen, einführen und systematisch studieren wird. In üblicher Rede von grammatischen Gesetzen denkt man hauptsächlich an die grammatischen Regeln einer bestimmten Sprache (Englisch, Deutsch, Chinesisch usw.), die konventionell bestimmt werden und von Sprache zu Sprache unterschiedlich sein können. Wenn man z.B. die Bedeutung „Sokrates und Platon“ auf Deutsch ausdrücken will, schreibt man den Ausdruck ,Sokrates und Platon“, während der Ausdruck „Sokrates Platon und“ in deutscher Sprache ungrammatisch ist. Im Lateinischen schreibt man „Sokrates Plato-que“, während der Ausdruck ,Sokrates que Plato“ ungrammatisch ist. Beide Sprachen haben also verschiedene grammatische Regeln, die Bedeutung „Sokrates und Platon“ auszudrücken. Ähnliche Unterschiede hinsichtlich der grammatischen Regeln von verschiedenen Sprachen sind überall zu zeigen.

Allerdings vertritt Husserl in den Logischen Untersuchungen (besonders in der $I V$. $L U)$ die Ansicht, dass es, außer den konventionell-grammatischen Regeln, auch einige apriorisch-grammatische Gesetze bestehen. Solche apriorisch-grammatischen Gesetze stammen nach Husserl nicht aus Konventionen, sondern gelten für alle Sprachen und sind durch Komplikationsmöglichkeiten von Bedeutungen zu erklären. Z.B. erlaubt weder Latein noch Deutsch noch irgendeine andere Sprache einen Ausdruck, der die Bedeutung ,,aber und Sokrates rot“ (durch,, aber und Sokrates rot“" auf Deutsch und ,,sed -que Sokrates roseus“ auf Latein) ausdrückt, denn sie ist gar keine einheitliche Bedeutung, die ein sinnvoller Ausdruck irgendeiner Sprache tragen kann.

In den Logischen Untersuchungen versucht Husserl, die Allgemeingültigkeit solcher apriorisch-grammatischen Gesetze durch seine originalen Ideen von „Bedeutungskategorie“ und ,reiner Grammatik“ zu charakterisieren: Nach Husserls Konzeption der „reinen Grammatik“ unterstehen Ausdrucksbedeutungen verschiedenen formalen Typen (= Bedeutungskategorien), die bestimmen, welche 
Bedeutungen sich mit welchen anderen auf welche Weisen $\mathrm{zu}$ einer einheitlichen Bedeutung zusammenschließen können. Im I. Abschnitt der vorliegenden Arbeit erkläre ich, wie Husserl die Konzeption der reinen Grammatik im Zusammenhang mit der empirischen Grammatik und der formalen Logik entwickelt; dabei werden die Begriffe der Bedeutungskategorien und der apriorisch-grammatischen Gesetze erörtert. Das führt zum Clou des Abschnitts - der Begriff des Grammatikalitätsphänomens wird bezüglich der Ausdrucksbedeutungen und deren Gegenstände eingeführt. Außerdem sind die Ideen von „Bedeutungskategorie“ und „reiner Grammatik“, wie der Appendix der vorliegenden Arbeit zeigt, interdisziplinär interessant für einige Logiker (wie Kazimierz Ajdukiewicz) und haben die kategoriale Grammatik bei Ajdukiewicz inspiriert.

Als eine phänomenologische Untersuchung studiert die vorliegende Arbeit das Grammatikalitätsphänomen von Ausdrucksbedeutungen auch im Zusammenhang mit Husserls phänomenologischer Theorie über Intentionalität. Deshalb treten wir im II. Abschnitt in Husserls transzendentale Phänomenologie ein und folgen den sogenannten noetisch-noematischen Konsitutionsanalysen. Dabei versuche ich, die Grundideen von Husserls transzendentaler Phänomenologie darzustellen und nötigenfalls (z.B. seinen Noemabegriff) kritisch zu rekonstruieren. Das ermöglicht es mir, den Begriff des Grammatikalitätsphänomens, der im I. Abschnitt entwickelt ist, im Rahmen der transzendentalen Phänomenologie zu reinterpretieren: Mittels der Begriffsbildungen, die um das zentrale Begriffsduo Noesis/Noema herum vorgenommen werden, lässt sich der Begriff des Grammatikalitätsphänomens systematisch auf Urteilsakte (und allgemeiner: Bedeutungsintentionen ${ }^{1}$ ) sowohl in noetischer als auch noematischer Hinsicht übertragen.

Im III. Abschnitt folge ich Husserls genetischen Analysen über das sogenannte Erkenntnisstreben, das durch die Stufen von Erfahrung und Urteilen hindurchgeht. Husserls genetische Analysen über die Erfahrung und das Urteilen zeigen, dass schon in der Erfahrung gewisse (vor-)grammatische Formbildungen als Vorgänger von

\footnotetext{
${ }^{1}$ Den Begriff der Bedeutungsintention werden wir im [§5] der vorliegenden Arbeit erörtern.
} 
Bedeutungskategorien konstituiert werden. Die Herausarbeitung solcher (vor-)grammatischen Formen innerhalb der Erfahrung wird es rechtfertigen, die Rede vom Grammatikalitätsphänomen vom Urteilen auf die Erfahrung und sogar Anschauung überhaupt zu erweitern. Im Schlusswort der vorliegenden Arbeit werde ich noch ein Stück weiter gehen: Mittels der von Husserl interpretierten brentanoschen These $^{2}$, dass nicht-objektivierende Akte auf objektivierenden Akten als Grundlage beruhen, wird die Rede vom Grammatikaliätsphänomen auf intentionale Akte überhaupt übertragen.

Deshalb wird die vorliegende Untersuchung - um mit den Worten von Jocelyn Benoist $^{3} \mathrm{zu}$ sprechen - die grammatikalische Strukturierung von Intentionalität systematisch charakterisieren. Insofern gilt die vorliegende Arbeit auch als eine spezielle Untersuchung über das allgemein phänomenologische Thema Intentionalität.

\footnotetext{
${ }^{2}$ Darüber vgl. [§4] der vorliegenden Arbeit.

${ }^{3}$ Jocelyn Benoist, „Grammatik und Intentionalität“, in: Edmund Husserl. Logische Untersuchungen, hrsg. von Verana Meyer, Akademie Verlag GmbH, Berlin 2008, S.123-137.
} 


\section{ABSCHNITT: \\ DAS GRAMMATIKALITÄTSPHÄNOMEN DER BEDEUTUNGEN}

\section{KAPITEL \\ VORBEREITENDE ANALYSEN}

\section{§1. Husserls Konzeption von „,reiner Grammatik“}

Die Konzeption der „,reinen Grammatik“ wurde zuerst in der IV. Logischen Untersuchung aufgestellt. Dieser Konzeption zufolge sind alle Sprachen, wie immer unterschiedlich sie aus historischen oder kulturellen Gründen sind, gewissen apriorischen Gesetzen unterworfen. Die Aufgabe der „reinen Grammatik“ besteht darin, diese apriorischen Gesetze systematisch zu studieren. Mit Husserls eigenen Worten, die Sprachen sind nur Einzelfälle eines von der reinen Grammatik bloßgelegten ,idealen

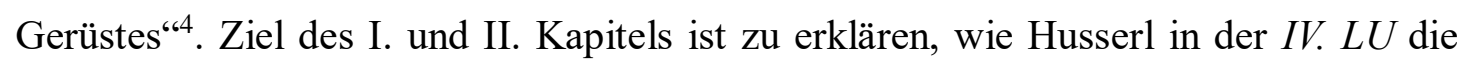
Ideen der Bedeutungskategorien und der apriorisch-grammatischen Gesetze sowie seine Konzeption der reinen Grammatik entwickelt.

\section{§2. Vorbereitende Analysen I: die Idealität der Bedeutungen}

Die IV. LU ist nicht unabhängig von den anderen Logischen Untersuchungen. Zur Verständigung der Idee der „reinen Grammatik“ ist es unentbehrlich, die Forschungsergebnisse anderer Logischen Untersuchungen als Vorbereitungen anzuführen. Hier möchte ich die Ergebnisse, die in den anderen Logischen Untersuchungen gewonnen werden und direkt relevant für die $I V . L U$ sind, kurz durch meine oder Husserls eigene Beispielsanalysen darstellen.

\footnotetext{
${ }^{4}$ Hua XIX/1: Logische Untersuchungen. Zweiter Band. Erster Teil. Untersuchungen zur Phänomenologie und Theorie der Erkenntnis, hrsg. von U. Panzer, Martinus Nijhoff, The Hague/Boston/Lancaster 1984, S.347.
} 
In der $I . L U$ hat Husserl mehrere wichtige Unterscheidungen gemacht: (1) die Unterscheidung zwischen Bedeutung und Ausdruck, (2) die Unterscheidung zwischen Bedeutung und Gegenstand, (3) die zwischen Bedeutung und Anschauungsbild, (4) die zwischen Bedeutung und bedeutunggebendem Akt sowie (5) die zwischen Bedeutung und anschaulich-bedeutungerfüllendem Akt. ${ }^{5}$

(1) Die Ausdrücke „Der Himmerl ist blau“, „The sky is blue“ und „,caelum caerulum est " sind verschieden, denn sie zu verschiedenen Sprachen gehören. Aber sie teilen dieselbe Bedeutung (Proposition). Das Identitätskriterium des Ausdrucks fällt also mit dem seiner Bedeutung nicht zusammen.

(2) Husserl schreibt: ,Jeder Ausdruck .... hat nicht nur seine Bedeutung, sondern er bezieht sich auch auf irgendwelche Gegenstände ... Niemals fällt aber der Gegenstand mit der Bedeutung zusammen. “6 Ein Beispiel dafür sind die Namen „der Sieger von Jena“ und „der Besiegte von Waterloo“ - sie haben verschiedene Bedeutungen (Frege'sche Sinne), aber sie beziehen sich auf denselben Gegenstand (Frege'sche Bedeutung). Deshalb muss man die Bedeutung des Ausdrucks von seinem Gegenstand unterscheiden.

(3) Bedeutung ist kein inneres Bild. Nach einer psychologistischen Auffassung ist die Bedeutung ein anschauliches Bild, das dem Subjekt vorschwebt, wenn es an einen Gegenstand denkt. ${ }^{7}$ Wenn man den Ausdruck ,Tausendeck“ ausspricht, schwebt ihm oft eine Figur mit unbestimmt vielen Seiten vor. Doch die inneren bildlichen Figuren des Tausendecks sind bei verschiedenen Denksubjekten verschieden, dagegen ist die Bedeutung von „Tausendeck“ identisch. Und ein Mathematiker kann sogar ohne ein inneres Bild die Bedeutung des Wortes ,Tausendeck“ besser verstehen als ein Laie, der ein anschauliches Bild verwertet. Aus diesem Grund muss man die Bedeutung von deren entsprechenden anschaulichen Bildern unterscheiden.

(4) Bedeutung ist kein intentionales Erlebnis, das für einen Ausdruck als bedeutunggebender $\mathrm{Akt}^{8}$ fungiert. Verschiedene Subjekte urteilen, dass die Summe der

\footnotetext{
${ }^{5} \mathrm{Zu}$ den Begriffen von bedeutunggebenden/bedeutungerfüllenden Akten und intentionalen Erlebenissen vgl. [§4] und [§5] der vorliegenden Arbeit.

${ }^{6}$ Hua XIX/1: Logische Untersuchungen, S.52.

${ }^{7}$ Ebd., S.67-72.

${ }^{8}$ Provisorisch kann man den Begriff des bedeutunggebenden Aktes wie folgt verstehen: Er ist ein intentionales
} 
beiden Kathetenquadrate in einem rechtwinkligen Dreieck gleich dem Hypothenusenquadrat ist. Dabei sind die Urteilsakte verschieden, doch die Urteilsbedeutung (bzw. die Proposition), der pythagorischer Satz, bleibt gegenüber den Urteilsakten identisch. Husserl zufolge sind die Urteilsakte reale Ereignisse, in der Zeit entstehend und vergehend, während die Urteilsbedeutung als ideale Entität zeitlos ist. ${ }^{9}$

(5) Bedeutung ist kein intentionales Erlebnis, das für einen Ausdruck als bedeutungerfüllender Akt fungiert. Die Bedeutung des Urteilsausdrucks „,der Himmel ist blau“ bezieht sich auf den Gegenstand, nämlich den Sachverhalt, dass der Himmel blau ist. Verschiedene Sprecher können aufgrund ihrer jeweiligen Anschauungsakte (Wahrnehmung oder Phantasie) das Urteil „,der Himmel ist blau“ fällen und den Aussagesatz „der Himmel ist blau“ behaupten. Aber einerseits sind die Anschauungsakte verschieden, während die Bedeutung des Ausdrucks identisch ist. Andererseits kann ein Hörer die Bedeutung des Ausdrucks „der Himmel ist blau“ verstehen, ohne den entsprechenden Anschauungsakt zu vollziehen. Aus diesen Gründen muss man die Ausdrucksbedeutung von den entsprechenden Anschauungsakten unterscheiden. ${ }^{10}$

Konklusion: Negativ gesprochen sind Bedeutungen weder identisch mit den sprachlichen Ausdrücken und seinen Gegenständen, noch mit den inneren Bildern, und noch mit den bedeutunggebenden/bedeutungerfüllenden Akten; positiv gesprochen sind die Bedeutungen ideale Entitäten gegenüber real-zeitlichen Entitäten wie den intentionalen Erlebnissen. ${ }^{11}$

\section{§3. Vorbereitende Analysen II: die Rede von Spezies}

In den Logischen Untersuchungen spricht Husserl nur von einer Art von idealen Entitäten, nämlich Spezies. Konsequenterweise hält er Bedeutung für ideale Spezies in den Logischen Untersuchungen. Seiner Meinung nach verhält sich die Bedeutung zu

\footnotetext{
Erlebnis, das einen Ausdruck sinnvoll macht. Ausführlich darüber vgl. [\$4] und [§5] der vorliegenden Arbeit.

${ }^{9}$ Hua XIX/1: Logische Untersuchungen, S.49-51.

${ }^{10}$ Ebd., S.62, 72-73.

${ }^{11}$ Näheres darüber vgl. Vittorio De Palma, „Husserls phänomenologische Semiotik“, in: Edmund Husserl. Logische Untersuchungen, S.43-58.
} 
den bedeutunggebenden Akten ebenso wie die Rot-Spezies zu den mannigfaltigen roten Dingen. ${ }^{12}$ Doch nicht alle Philosophen sind sich einig, ideale Spezies zu akzeptieren. In $I I . L U$ versucht Husserl, die nominalistischen Theorien über Spezies zu kritisieren und den ontologischen Status von idealer Spezies oder idealem Wesen zu begründen.

Peter Simons hat die Begründungsstrategie der II. LU unter drei Titeln zusammengefasst: (1) ontologische Begründung, (2) phänomenologische Begründung sowie (3) Kritik der Abstraktionstheorien. ${ }^{13}$

(1) Spezies dienen dazu, die Ähnlichkeiten und Unähnlichkeiten der Gegenstände zu erklären. Es seien zum Beispiel A eine rote Kugel, B ein roter Würfel. A und B sind gleich(ähnlich) in der Farbe, aber nicht gleich(ähnlich) in der Gestalt. Husserl zufolge lässt sich die Gleichheit(Ähnlichkeit) in Hinsicht auf die Farbe dadurch erklären, dass beide die Rot-Spezies instanziieren. Er schreibt, „Gleichheit ist das Verhältnis der Gegenstände, welche einer und derselben Spezies unterstehen. Ist es nicht mehr erlaubt, von der Identität der Spezies zu sprechen, von der Hinsicht, in welcher Gleichheit statthat, so verliert auch die Rede von Gleichheit ihren Boden." 14 Um das Ähnlichkeitsverhältnis zwischen Dingen zu erklären, muss man also ontologisch die Spezies akzeptieren, die als ideale Einheit (z.B. die Spezies Rot) gegenüber den mannigfaltigen Einzeldingen (rote Dinge) oder ihren Einzelmomenten (Rotmomente der Dinge) identisch ist - das ist Husserls ontologische Begründung für die Annahme der idealen Spezies.

(2) Husserl zufolge sind die Bewußtseinsweise, in der ein Individuelles gegeben ist, und die, in der eine Spezies gegeben ist, evident verschieden. Er schreibt:

Wir brauchen bloß auf die Fälle zurückzugehen, in welchen sich individuelle oder spezifische Vorstellungen intuitiv erfüllen ... dass der Akt, in dem wir Spezifisches meinen, in der Tat wesentlich verschieden ist von demjenigen, in

\footnotetext{
12 „Die Bedeutung verhält sich also zu den jeweiligen Akten des Bedeutens ... wie etwa die Röte in specie zu den hier liegenden Papierstreifen, die alle diese selbe Röte „haben“. “Hua XIX/1: Logische Untersuchungen, §§31-33.

13 Vgl. Peter Simons, „Zugang zum Idealen: Spezies und Abstraktion“, in: Edmund Husserl. Logische Untersuchungen, S.77-91. Es ist zu bemerken, dass Peter Simons ontologische Begründung und phänomenologische Begründung in Husserls Phänomenologievoneinander kaum zu trennen sind. Denn in vielen Fällen in Husserls Phänomenologie entspricht einem Unterschied zwischen den Bewußtseinsweisen oder Konstitutionsweisen (Stichwort: Konstitution, davon im II. Abschnitt der vorliegenden Arbeit noch zu sprechen) von Gegenständen ein Unterschied zwischen den ontologischen Kategorien von ihnen (z.B. physische Gegenstände vs. Menschen).

${ }^{14}$ Hua XIX/1: Logische Untersuchungen, S.118.
} 
dem wir Individuelles meinen ... Beiderseits erscheint ja dasselbe Konkretum, und indem es erscheint, sind beiderseits dieselben sinnlichen Inhalte in derselben Auffassungsweise gegeben ... Das eine Mal ist die Erscheinung die Vorstelllungsgrundlage für einen Akt individuellen Meinens, d.h. für einen solchen Akt, in dem wir in schlichter Zuwendung das Erscheinende selbst, dieses Ding oder dieses Merkmal, dieses Stück im Dinge meinen. Das andere Mal ist sie Vorstellungsgrundlage für einen Akt spezialisierenden Auffassens und Meinens; d.h. während das Ding, oder vielmehr das Merkmal am Dinge erscheint, meinen wir nicht dieses gegenständliche Merkmal, dieses Hier und Jetzt, sondern wir meinen seinen Inhalt, seine „Idee“; wir meinen nicht dieses Rotmoment am Hause, sondern das Rot. ${ }^{15}$

Dieser Unterschied der Bewußtseinsweisen reflektiert sich in der Sprache. In der Alltagssprache verwendet man Aussagen wie „Rot ist eine Farbe“, womit man nicht von dem individuellen Moment ${ }^{16}$ eines individuellen Gegenstandes spricht (z.B. dem Rotmoment dieses Autos), sondern explizit über eine Entität, die durch die Mannigfaltigkeit der entsprechenden individuellen Dinge oder dinglichen Momente instanziiert wird. Husserl zufolge kann man es nicht leugnen, „daß wir in distinktem Sinn von Spezies sprechen, daß wir in unzähligen Fällen nicht das Einzelne, sondern seine Idee meinen und nennen, daß wir über dieses ideal Eine als Subjekt genau so Aussagen machen können, wie über das individuell Einzelne. “17

Durch diese Analysen über die phänomenologischen und sprachlichen Verschiedenheiten zwischen Bewußtsein des Allgemeinen und Bewußtsein des Individuellen glaubt Husserl, die Annahme der Spezies als idealer Entität durch eine phänomenologische Begründung gerechtfertigt zu haben.

(3) Außerdem kritisiert Husserl in II. LU die Abstraktionstheorien (Locke, Mill, Berkeley, Hume). Diese Abstraktionstheorien versuchen, das Allgemeinheitsbewußtsein auf das Bewußtsein des Individuellen zu reduzieren und

\footnotetext{
${ }^{15}$ Ebd., S.114.

${ }^{16}$ D.i. ein unselbständiger Bestandteil des individuellen Gegenstandes. Darüber vgl. [§6] der vorliegenden Arbeit.

${ }^{17}$ Hua XIX/1: Logische Untersuchungen, S. 160.
} 
somit die Spezies qua ideale Einheit abzulehnen. Husserls Kritik zeigt, dass diese Abstraktionstheorien jeweils ihre schwachen Punkte haben. Hier möchte ich die Details von Husserls Kritik übergehen, denn sie sind für uns nur von Nebeninteresse und haben weniger Originalität. Es genügt darauf hinzuweisen, dass Husserls Strategie ist, die Schwächen der gegnerischen Theorien aufzuzeigen und somit seine eigene Theorie in einer negativen Weise indirekt zu verteidigen.

Wie Peter Simons bewertet, sind Husserls Begründungen für die Annahme der idealen Spezies, die in II. $L U$ entwickelt wurden, nicht wasserdicht. ${ }^{18}$ In der vorliegenden Arbeit kommt es aber nur darauf an, zu verstehen, wozu diese Annahme der idealen Spezies im Rahmen der Logischen Untersuchungen dient. In den Prolegomena kritisiert Husserl den logischen Psychologismus, der das Vorurteil ${ }^{19}$ hegt, dass die Gegenstände, die die Logik untersucht, subjektiv-psychische Phänomene (Vorstellen, Urteilen, Schließen usw.) innerhalb des Bewußtseins sind. Husserl zufolge würde dieses Vorurteil zum logischen Empirismus ${ }^{20}$ und sogar Skeptizismus ${ }^{21}$ führen, was für ihn unakzeptierbar ist. ${ }^{22}$ Diesem psychologistischen Vorurteil gegenüber spricht Husserl sich für die Ansicht aus, dass die Logik nicht die psychischen Akte selber, sondern ihre idealen Inhalte, nämlich Bedeutungen zum Forschungsgebiet hat. ${ }^{23}$ In den Logischen Untersuchungen hat Husserl nur eine Art ideale Entität im Auge, nämlich Spezies oder Wesen, zu der auch sprachliche Bedeutungen ${ }^{24}$ gehören. ${ }^{25}$ Das Ziel der II. $L U$ ist also, den ontologischen Status der Bedeutungen durch eine allgemeinere Untersuchung der Spezies zu verstärken. Konsequenterweise bildet die

\footnotetext{
${ }^{18}$ Vgl. Peter Simons, „Zugang zum Idealen: Spezies und Abstraktion“, in: Edmund Husserl. Logische Untersuchungen, S.77-91.

${ }^{19}$ Hua XVIII: Erster Band. Prolegomena zur reinen Logik, hrsg. von Elmar Holenstein, Martinus Nijhoff, Den Haag 1975, S.170-171.

${ }^{20}$ Das ist die Ansicht, dass logische Gesetze Naturgesetze des Psychischen und deshalb empirische Gesetze sind. Demzufolge wären logische Gesetze nicht a priori gültig ließen sich nur durch Induktion aus einzelnen psychologischen Tatsachen rechtfertigen. Vgl. Hua XVIII: Prolegomena zur reinen Logik, S.72-87.

${ }^{21}$ Das ist die Ansicht, dass logische Gesetze nur für gewisse Individuen oder Spezies von denkenden Wesen gültig sind. Dies nennt Husserl auch Relativismus. Vgl. Hua XVIII, VII. Kapitel.

${ }^{22}$ Mehr darüber vgl. Richard Tieszen, „Husserl's Concept of Pure Logic“, und Robert Hanna, „Husserls's Arguments against Logical Psychologism“", in: Edmund Husserl. Logische Untersuchungen, S.9-26, $27-42$.

${ }^{23}$ Hua XVIII: Prolegomena zur reinen Logik, S.177-180.

${ }^{24}$ Nicht im Sinne von allgemeiner Bedeutungsfunktion (oder Charakter bei David Kaplan), sondern im Sinne von jeweiliger Bedeutung (Ausdrucksbedeutung). Vgl. Hua XIX/1, S.85-92. Darüber unten im [§5]. Zu einer kurzen, aber klaren Erörterung der Begriffe von allgemeiner Bedeutungsfunktion und jeweiliger Bedeutung vgl. Christian Beyer, Intentionalität und Referenz. Eine sprachanalytische Studie zu Husserls transzendentaler Phänomenologie, mentis Verlag GmbH, Paderborn 2000, S.72-76.

${ }^{25}$ Hua XIX/1: Logische Untersuchungen, S.108, 352.
} 
Idealität der Bedeutungen eine Grundprämisse der IV. Logischen Untersuchung.

\section{§4. Vorbereitende Analysen III: intentionale Erlebnisse}

In den $V$. und $V I$. $L U$ entwickelt Husserl seine Phänomenologie der intentionalen Erlebnisse. Dabei (1) beschreibt Husserl den Wesenscharakter der intentionalen Erlebnisse, und (2) analysiert ihre inneren Momente sowie (3) klassifiziert intentionale Erlebniesse in verschiedene Gattungen und Arten.

(1) Husserl schreibt, ,In der Wahrnehmung wird etwas wahrgenommen, in der Bildvorstellung etwas bildllich vorgestellt, in der Aussage etwas ausgesagt, in der Liebe etwas geliebt, im Hasse etwas gehaßt, im Begehren etwas begehrt usw." ${ }^{26}$ Das Gemeinsame, das an solchen Beispielen zu erfassen ist, ist Intentionalität, nämlich dassich-auf-Gegenständlichkeit-Beziehen. Bei Husserls Terminologie intentionales Erlebnis oder Akt handelt es sich eben um die ,psychische Phänomene“, die den Charakter der Intentionalität haben. ${ }^{27}$ Beispiele von intentionalen Erlebnissen oder Akten sind Wahrnehumg, Vorstellung, Liebe, Wunsch usw.

(2) Verschiedene Akte beziehen sich auf die Gegenständichkeit in verschiedenen Weisen. In der $V . L U$ erklärt Husserl die Unterschiede, ,in der Weise der gegenständlichen Beziehung ${ }^{، 28}$ durch zwei innere Momente des intentionalen Aktes, nämlich (2a) Aktqualität und (2b) Aktmaterie.

(2a) Jedes intentionale Erlebnis gehört zu einem bestimmten Akttyp (kind of act ${ }^{29}$ ), es ist entweder Urteilen oder Wünschen oder Lieben usw. Den verschiedenen Akttypen entsprechen verschiedene Weisen, in denen die intentionalen Erlebnisse ihre Gegenstände intendieren. In diesem Sinne intendiert das Urteil, dass das Wetter gut ist und der Wunsch, dass das Wetter gut ist, denselben Sachverhalt als ihren Gegenstand, aber in verschiedenen Weisen - im Urteil ist der Sachverhalt geurteilt, im Wunsch ist er gewünscht. Husserl zufolge ist der Akttyp eines intentionalen Erlebnisses durch ein inneres Moment bestimmt und er nennt dieses Moment Aktqualität. Wenn mehrere Akte gleiche Aktqualitäten haben, dann gehören sie zu demselben Akttyp. ${ }^{30}$

\footnotetext{
${ }^{26}$ Hua XIX/1: Logische Untersuchungen, S.380.

${ }^{27}$ Ebd., S.382, 392, 427.

${ }^{28}$ Ebd.

${ }^{29}$ Ebd., S.425-426. Dazu vgl. auch David Woodruff Smith and Ronald McIntyre, Husserl and Intentionality. A Study of Mind, Meaning, and Language, D. Reidel Publishing Company, Dordrecht/Boston/Lancaster 1982, S.113-114.

${ }^{30}$ Smith und McIntyre haben es unterlassen, genauer zu erörtern, inwieweit die Qualitäten die Akttypen (kind of act) bestimmen. Aktqualitäten differenzieren Akttypen in zwei Gattungen, nämlich die Gattungen von objektivierenden Akten und nicht-objektivierenden Akten, von denen wir unten in diesem Paragraphen noch sprechen werden.
} 
Es ist zu bemerken, dass Husserl manchmal von derselben Qualität verschiedener Akte redet. ${ }^{31}$ Diese Rede sollte man vorsichtig aufnehmen. Wenn z.B. Urteilsakte A und $\mathrm{B}$ individuell verschieden sind, dann sind ihre jeweiligen Aktqualitäten als innere Momente auch individuell verschieden; streng genommen haben sie nicht dieselbe Aktqualität, sondern gleiche Aktqualitäten, oder, sie haben dieselbe Qualität als Spezies. Dasselbe gilt auch für Husserls Rede von derselben Aktmaterie, damit ist nicht die Materie als individuelles Moment, sondern Materie als Spezies oder ideale Materie gemeint.

(2b) Die Qualität ist nicht der einzige Faktor, der die Weise der gegenständlichen Beziehung eines intentionalen Erlebnisses bestimmt. Das Urteil, dass das Wetter gut ist, und das Urteil, dass das Auto rot ist, haben dieselbe Qualität (als Spezies), aber sie intendieren verschiedene Gegenstände und haben natürlich auch verschiedene Weisen der gegenständlichen Beziehung. Diese Verschiedenheit der beiden Akte erklärt Husserl durch den Begriff von Aktmaterie. Materie ist auch ein inneres Moment des Aktes, genauer gesagt, sie ist dasjenige Moment im Akte, ,was ihm die bestimmte Richtung auf ein Gegenständliches verleiht" und ,,was ihm die Richtung gerade auf diesen und keinen anderen Gegenstand erteilt" ${ }^{32}$. Die Materie bestimmt, auf welche Gegenständlichkeit der betreffende Akt sich bezieht. Und die Verschiedenheit der beiden Urteilsakte im Beispiel lässt sich phänomenologisch dadurch erklären, dass sie verschiedene Aktmaterien (als Spezies) haben.

Die Materie bestimmt nicht nur, auf welche Gegenständlichkeit der Akt sich bezieht, sondern auch, als was er sie auffasst. ${ }^{33}$ Akte, die denselben Gegenstand intendieren, können noch verschiedene Materien (als Spezies) haben. Das erörtert Husserl durch Beispiele von äquivalenten Vorstellungen wie ,das gleichseitige Dreieck“ und „,das gleichwinklige Dreieck“. In diesem Sinne ist die Materie ,die im phänomenologischen Inhalt des Aktes liegende Eigenheit desselben, die es nicht nur bestimmt, daß der Akt die jeweilige Gegenständlichkeit auffaßt, sondern auch als was er sie auffaßt, welche Merkmale, Beziehungen, kategorialen Fromen er in sich selbst

Innerhalb der nicht-objektivierenden Akte differenzieren Aktqualitäten die Akttypen weiter in verschiedene Arten wie Wunsch, Liebe, Hoffnung usw. Aber innerhalb der objektivierenden Akte sind für die Unterscheidung von verschiedenen Aktarten wie Wahrnehmung, Imagination, Signifikation ein anderes inneres Moment des Aktes (d.i. die Repräsentation) verantwortlich. Näheres darüber vgl. [§35] der vorliegenden Arbeit.

${ }^{31}$ Z.B. in Hua XIX/1, S.435,497.

${ }^{32}$ Hua XIX/1: Logische Untersuchungen, S.428.

${ }^{33}$ Ebd., S.429-430: ,Danach muß uns die Materie als dasjenige im Akte gelten, was ihm allererst die Beziehung auf ein Gegenständliches verleiht, und zwar diese Beziehung in so vollkommener Bestimmtheit, daß durch die Materie nicht nur das Gegenständliche überhaupt, welches der Akt meint, sondern auch die Weise, in welcher er es meint, fest betimmt ist." 
ihr zumißt. “34

Qualität und Materie sind im folgenden Sinne voneinander abhängig: es gibt keine Qualität, die nicht mit einer Materie verknüpft ist, und umgekehrt auch keine Materie, die sich nicht mit einer Qualität verknüpft (Hua XIX/1, 430). Da die Einheit von Qualität und Materie das wesentliche Bestandstück eines intentionalen Aktes bildet, bezeichnet Husserl diese Einheit als das intentionale Wesen des Aktes. ${ }^{35}$

(3) Mithilfe des Begriffspaars von Qualität/Materie klassifiziert Husserl intentionale Erlebnisse in verschiedene Gattungen und Arten. Wenn man sich zuerst nach der Aktqualität orientiert, dann kann er zwischen objektivierenden und nichtobjektivierenden Akten unterscheiden.

Husserl knüpft seine Unterscheidung zwischen objektivierenden und nichtobjektivierenden Akten an den brentanoschen Satz an. Der brentanosche Satz lautet: Jedes intentionale Erlebnis ist entweder eine Vorstellung, oder beruht auf Vorstellungen als seiner Grundlage. ${ }^{36}$ Husserl glaubt, dass gewisse Evidenz in diesem Satz liegt, und es kommt darauf an, wie man diese Evidenz genauer charakterisiert und was man mit dem Wort „Vorstellung“ im brentanoschen Satz meint (a.a.O., 447). Dafür verwendet Husserl seine Terminologie von Aktqualität/Aktmaterie.

Husserl zufolge haben alle intentionalen Erlebnisse Materie, aber nicht alle intentionalen Erlebnisse besitzen die Materie in direkter Weise. Eine Freude z.B. erhält seine Materie nur dadurch, dass eine andere Aktqualität (z.B. Qualität eines entsprechenden Urteilsaktes), die mit einer Materie verknüpft ist, die Qualität der Freude fundiert. Dabei wird die Materie der fundierenden Aktqualität zugleich Materie der Freudequalität. Im Vergleich dazu besitzen manche Aktqualitäten direkt Materie, ohne auf einer anderen Qualität fundiert zu sein. Akte, deren Qualität nur durch das Fundierungsverhältnis eine Materie gewinnt, bezeichnet Husserl als nichtobjektivierende Akte; Akte, deren Qualität die Materie direkt besitzt, bezeichnet er als objektivierende Akte. Beispiele der ersteren sind Wunsch, Liebe, Hoffnung, usw. Beispiele der letzteren sind Wahrnehmung, Phantasie, Urteil usw. (a.a.O., 514-518)

Mithilfe der obigen Analyse kann man den brentanoschen Satz wie folgt interpretieren: Jedes intentionale Erlebnis ist entweder ein objektivierender Akt, oder

\footnotetext{
${ }^{34}$ Ebd.

${ }^{35}$ Ebd., S.431: „Sofern uns nun (wie wir hören werden) Qualität und Materie als die durchaus wesentlichen und daher nie zu entbehrenden Bestandstücke eines Aktes gelten müssen, würde es passend sein, die Einheit beider ... als das intentionale Wesen des Aktes zu bezeichnen."

${ }^{36}$ Ebd., S.383: „Eine zweite für uns wertvolle Bestimmung der psychischen Phänomene faßt Brentano dahin, „daß sie entweder Vorstellungen sind oder auf Vorstellungen als ihrer Grundlage beruhen“ “.. " Vgl. auch S.443.
} 
beruht auf objektivierenden Akten als seiner Grundlage. ${ }^{37}$

Innerhalb der Gattung der objektivierenden Akte kann man die Klassifikation weiter fortsetzen. Wenn man sich dabei nach der qualitativen Verschiedenheit orientiert, dann ergibt sich (3a) die Unterscheidung zwischen setzenden und nicht-setzenden objektivierenden Akten; wenn man sich nach der Verschiedenheit in Hinsicht auf die Materie orientiert, dann ist (3b) die Unterscheidung zwischen nominalen und propositionalen objektivierenden Akten zu konstatieren. ${ }^{38}$

(3a) Ein setzender objektivierender Akt intendiert seinen Gegenstand als wirklich seienden, während ein nicht-setzender Akt das Sein seines Gegenstandes dahingestellt lässt - er stellt den Gegenstand bloß vor, ohne über sein Wirklich-sein zu entscheiden. ${ }^{39}$ Wenn man z.B. eine Aussagesatz von anderen versteht und zugleich den geurteilten Sachverhalt für wirklich hält, dann vollzieht er ein setzendes Urteil; wenn er den Satz nur versteht, ohne mitzuurteilen und sogar sich jeder Stellungnahme enthält, dann vollzieht er eine bloße Vorstellung (eine nicht-setzende Variante des Urteils) des betreffenden Sachverhalts; beide Akte haben dieselbe Materie, aber verschiedene Qualitäten (setzende vs. nicht-setzende). Jedem Urteil entspricht eine solche nichtsetzende Variante. Für Husserl gilt das allgemein für alle objektivierenden Akte: Jedem setzenden objektivierenden Akt entspricht ein nicht-setzender objektivierender Akt von derselben Materie, und umgekehrt. ${ }^{40}$ (Es lässt sich noch diskutieren, inwieweit diese Allgemeinheit gilt. ${ }^{41}$ )

(3b) Husserl zufolge lassen sich die objektivierenden Akte in nominale und

\footnotetext{
${ }^{37}$ Husserl diskutiert noch andere Lesarten des Satzes. Eine davon beruht auf der Äquivokation des Vorstellungsbegriffs: Jedes intentionale Erlebnis ist entweder eine bloße Vorstellungsakt (= ein Akt ohne den sogenannten belief-Charakter, wie das bloße Verständnis eines Aussagesatzes ohne Überzeugung), oder beruht auf einer oder mehreren Vorstellungen (= Materie, die nur ein Aktmoment, aber kein vollständiger Akt bildet). (Vgl. $\S \S 26-32$ der $V . L U$ ) Eine andere Lesart versteht unter dem Vorstellungsbegriff nominalen (objektivierenden) Akt (darüber unten in diesem Paragraphen) und lautet: Jeder Akt ist entweder selbst eine Vorstellung (= ein nominaler Akt) oder er ist in einer oder mehreren Vorstellungen (= nominalen Akten) fundiert. (Vgl. §33-36 der V. LU)

${ }^{38}$ Hua XIX/1: Logische Untersuchungen, S.500-501.

${ }^{39}$ In Ideen I unterscheidet Husserl zwei Sorten von Akten ohne belief-Entscheidung: Einerseits die sogenannten doxischen Modalitäten, die die Existenz des Gegenstandes nur als möglich, fraglich, wahrscheinlich usw. setzen, andererseits die Neutralitätsmodifikation, die alle Setzung gleichsam entkräftet. Näheres darüber vgl. [§37] der vorliegenden Arbeit.

${ }^{40}$ Hua XIX/1: Logische Untersuchungen, S.499-500, 505, 507.

${ }^{41}$ Husserl ist der Meinung, dass jedem setzenden objektivierenden Akt ein nicht-setzender objektivierender Akt entspricht, der sich von ihm nur hinsichtlich des Setzungscharakters unterscheidet. (Hua XIX/1, S.507) Z.B. ist das nicht-setzende Gegenstück einer setzenden Phantasie auch eine Phantasie, denn beide veranschaulichen den Gegenstand phantasiemäßig (vgl. [\$35] der vorliegenden Arbeit). Aber hat eine Wahrnehmung ein solches Gegenstück? Wahrnehmung ist ein setzender objektivierender Akt, in dem der Gegenstand „selbst“ und „leibhaft“ gegeben wird. Man möchte fragen, gibt es nicht-setzende Wahrnehumg, in der der Gegenstand nicht für wirklich gehalten wird, obwohl er selbst und leibhaft gegeben ist? Die beiden Faktoren, den-Gegenstand-dahingestellt-Lassen und den-Gegenstand-leibhaft-Haben, scheinen imkompatibel zu sein. Ist das nicht-setzende Gegenstück einer Wahrnehmung nicht vielmehr eine Phantasie? Diese Fragen hat Husserl in den Logischen Untersuchungen auch berührt, aber nicht ausführlich behandelt (vgl. Hua XIX/1, 511-512).
} 
propositionale Akte einteilen. Nominal sind diejenigen objektivierenden Akte, die durch Namen ${ }^{42}$ ausgedrückt ${ }^{43}$ werden können; propositional sind diejenigen, die durch Sätze ausdrückbar sind. Mit einem Wort, nominale Akte und propositionale Akte haben verschiedene Ausdrücke.

Husserl erklärt diese Verschiedenheit in Bezug auf die Ausdrückbarkeit durch die Verschiedenheit der Weise, in der ein nominaler oder propositionaler Akt seinen Gegenstand intendiert: Metaphorisch gesagt intendiert ein nominaler Akt seinen Gegenstand monothetisch oder einstrahlig, während ein propositionaler Akt den Gegenstand synthetisch oder mehrstrahlig intendiert. ${ }^{44}$ Das folgende Beispiel kann den Unterschied zwischen einstrahligem und mehrstrahligem Intendieren illustrieren.

Ein Urteilsakt ,,der Himmel ist blau“ intendiert den Sachverhalt, dass der Himmel blau ist. Dabei wird eine Synthesis vollzogen: Der Urteilsakt verkünupft zwei Vorstellungen („der Himmel“ und „blau“ ) in der prädikativen Form, damit ein Sachverhalt als sein Gegenstand konstituiert wird. Mit Husserls eigenen Worten, „Eine Thesis wird vollzogen und daraufhin eine zweite unselbständige Thesis, derart, daß in der Aufeinandergründung dieser Thesen die synthetische Einheit des Sachverhalts zu intentionaler Konstitution kommt. “45

Aber man kann auch denselben Sachverhalt monothetisch intendieren. In der Sprache verwendet man oft Nebensätze, um einen Sachverhalt, der original in einem Urteilsakt konstituiert ist, zu nennen oder monothetisch zu intendieren. Man sagt „dass der Himmel blau ist, ist erfreulich“. Husserl zufolge ist der Nebensatz „dass der Himmel blau ist" eigentlich kein Satz, der einen Sachverhalt urteilend intendiert, sondern ein Name, der ihn nennt. Eine äquivalente Umformulierung des Satzes, die keine Nebensätze enthält, kann die Sachlage klarer machen:,,Der Himmel ist blau - dies ist erfreulich“; das dies „weist auf den ausgesagten Sachverhalt wie mit dem Finger hin“ (Hua XIX/1, 492). Bei dem Akt, der durch den Nebensatz oder das dies ausgedrückt wird, braucht keine Synthesis vollzogen zu werden. Husserl gesteht, dass der dabei entsprungene monothetische Akt (,dass der Himmel blau ist“) auf den ursprünglicheren synthetischen Akt („der Himmel ist blau“) zurückweist, und dass solche Umwandlung der synthetischen Vielstrahligkeit in eine nominale Einstrahligkeit

\footnotetext{
${ }^{42}$ Namen in einem weiten (an John S. Mill anknüpfenden) Sinn, wonach auch allgemeine Namen wie ,ein A“ und Nebensätze, die an die Subjektstelle eines Satzes gesetzt werden können, wie „dass der Reichstag geöffnet ist“, Namen sind.

${ }^{43}$ Ausgedrücktsein in Sinnen von (ii) oder (iii) im [§5].

${ }^{44}$ Hua XIX/1: Logische Untersuchungen, S.492.

${ }^{45}$ Ebd.
} 
bei allen synthetischen Akten möglich ist, deshalb scheinen die beiden Akte miteinander nah verwandt zu sein (a.a.O., 501-502). Allerdings schreibt er, „,mit dieser Rede vom Entspringen und Zurückweisen ist schon gesagt, daß die einen und anderen [Namen und Urteile] verschieden sind.“46

In diesem Sinne intendieren nominale und propositionale Akte ihre Gegenständlichkeit, und manchmal sogar dieselbe Gegenständlichkeit, in verschiedenen Weisen (monothetisch vs. synthetisch). Husserl erklärt diesen Unterschied durch die Eigenheit der Aktmaterie. Die Aktmaterien lassen sich in nominale (monothetische) und propositionale (synthetische) einteilen. Wenn die Gesamtmaterie eines objektivierenden Aktes nominal ist, dann ist er ein nominaler Akt und intendiert seinen Gegenstand einstrahlig; wenn die Gesamtmaterie propositional ist, dann intendiert er den Gegenstand mehrstrahlig.

Es ist zu bemerken, dass die prädikative Synthesis nur eine besondere Form der propositionalen Akte ist. Es gibt auch Synthesen anderer kategorialen Formen, z.B. kollektive Synthesis wie „Anna und Peter und Smith“. Bei Husserl wird jeder objektivierende Akt, der eine (syntaktische) Synthesis irgendwelcher kategorialen Form vollzieht, in die Art des propositionalen Aktes eingeordnet. (vgl. Hua XIX/1, S.501)

Die Unterscheidung von setzenden/nicht-setzenden objektivierenden Akten und die von nominalen/propositionalen Akten kreuzen sich. Deshalb kann man die objektivierenden Akte in vier Arten klassifizieren: (i) setzende nominale Akte ( wie die signitive Vorstellung „Göttingen“), (ii) nicht-setzende nominale Akte (wie die Phantasie „Pegasus“), (iii) setzende propositionale Akte (das Urteil , $1+1=2$ ") sowie (iv) nicht-setzende propositionale Akte (z.B. das Verstehen des Satzes „Napoleon entdeckt Amerika“ bei Lektüre eines Romans).

\section{§5. Vorbereitende Analysen IV: Bedeutungsintention und Bedeutungserfüllung}

Ein sinnlicher Wortlaut (ausgesprochen oder geschrieben) als physischer Gegenstand hat keine Bedeutung und ist kein richtiger Ausdruck. Husserl schreibt, „,das geschriebene Wort, ist ... ein physisches Objekt so gut wie irgendein beliebiger Federzug oder Tintenfleck auf dem Papier; es ist uns also in demselben Sinne wie

\footnotetext{
${ }^{46}$ Ebd., S.486.
} 
irgendein physisches Objekt sonst ,gegeben“ “. ${ }^{47}$ Korrelativ ist der Akt, der den sinnlichen Wortlaut intendiert, eine bloße Wahrnehmung wie jede Wahrnehmung von physischen Dingen.

Was den Wortlaut zum Ausdruck macht, ist das Subjekt, das ihn als einen sprachlichen Ausdruck verwendet. Genauer gesprochen muss das Subjekt neben der Wortlaut-Wahrnehmung noch einen anderen Akt vollziehen, der dem Wortlaut die Bedeutung verleiht, damit er als ein richtiger Ausdruck fungieren kann. Husserl bezeichnet diesen Akt als bedeutungverleihenden/sinngebenden Akt oder Bedeutungsintention $^{48}$. Bedeutungsintention ist oft ein setzender Akt, z.B. ein Urteil, das das Subjekt vollzieht, wenn es einen Aussagesatz aufrichtig behauptet; sie kann auch eine nicht-setzender Akt sein, wenn z.B. ein Schriftsteller einen Roman schreibt.

Michael Dummett ist der Meinung, dass Husserls Begriff des bedeutungverleihenden Aktes oder der Bedeutungsintention einer Humpty-DumptyTheorie zu nahe steht. ${ }^{49}$ Im Gespräch mit Alice verwendet Humpty Dumpty das Wort „Glory“, er meint damit aber nicht Ehre, sondern seltsamerweise schlagendes Argument. Als Alice entgegnet, dass ,Glory“ schlagendes Argument nicht bedeutet, erwidert er mit der Humpty-Dumpty-Theorie: Ein Ausdruck bedeutet genau das, was sein Verwender (Sprecher) will. ${ }^{50}$ Dagegen wendet Dummett ein, dass es nicht so ist, wie die Humpty-Dumpty-Theorie denkt, als ob die Bedeutung eines Ausdrucks durch die Willkür des Sprechers bestimmt wäre. Dummett zufolge muss man die soziale Dimension der Sprache respektieren: Man begegnet der Bedeutung eines Ausdrucks nicht dadurch, dass er dem Ausdruck eine vorhandene Bedeutung verleiht, sondern er erwirbt sie beim Lernen der betreffenden Sprache, deren Regeln durch soziale Konventionen innerhalb einer Sprachgemeinschaft festgelegt sind. Die HumptyDumpty-Theorie hat diese soziale Dimension, die für Dummett die grundlegendere sind,

\footnotetext{
${ }^{47}$ Hua XIX/1: Logische Untersuchungen, S.420.

${ }^{48}$ Ebd., S.44.

49 Michael Dummett, Origins of Analytical Philosophy, Bloomsbury Academic, London/New Delhi/New York/Sydney 2014, S.41ff. ,The meaning-conferring is a myth. Humpty Dumpty's theory was wrong, and Husserl came too close to adopting it."

${ }^{50}$ Vgl. Lewis Carroll, Through the Looking Glass, Kap. VI: ,, 'When I use a word,' Humpty Dumpty said in rather a scornful tone, 'it means just what I choose it to mean - neither more nor less.' “'
} 
vernachlässigt. $^{51}$

Hat Husserls Begriff der Bedeutungsintention auch die soziale Dimension von sprachlichen Ausdrücken unterlassen, wie es bei Humpty-Dumpty-Theorie der Fall ist? Die Sachlage ist nicht so einfach, wie Dummetts Kritik konzipiert. Husserl unterscheidet zwischen zwei Ebenen von sprachlichen Bedeutungen: allgemeiner Bedeutungsfunktion und jeweiliger Bedeutung, diese Unterscheidung tritt bei indexikalischen Ausdrücken (wesentlich okkasionellen Ausdrücken bei Husserl) klar hervor. Vergleichen wir die folgenden Ausdrücke (angenommen, dass A und B verschiedene Personen sind):

A sagt: (A) Ich habe Blutgruppe A. - Sachverhalt [A hat Blutgruppe A]

B sagt: (B) Ich habe Blutgruppe A. - Sachverhalt [B hat Blutgruppe A]

Im Sinne von allgemeiner Bedeutungsfunktion haben die ,ich“-Ausdrücke in Sätzen A und B dieselbe sprachliche Bedeutung (oder denselben Charakter bei Kaplan). Allerdings sind ihre sprachlichen Bedeutungen im Sinne von jeweiliger Bedeutung (Ausdrucksbedeutung) unterschiedlich, ${ }^{52}$ denn die Gegenstände, auf die sich die beiden , ich“-Ausdrücke beziehen, sind verschieden. Repräsentieren wir die allgemeine Bedeutungsfunktion des Ausdrucks (wie ,ich“) als $a($ ) und den jeweiligen Äußerungskontext als $k$. Dann bekommen wir die jeweilige Bedeutung $a(k)$ dadurch, dass wir den Kontext $k$ als Argument in die allgemeine Bedeutungsfunktion $a($ ) eingeben. Daher können wir die jeweiligen Bedeutungen der ,ich“-Ausdrücke in A und B jeweils als $a\left(k_{A}\right)$ und $a\left(k_{B}\right)$ repräsentieren und somit voneinander unterscheiden. Bei Ausdrücken (wie Eigennamen), die nicht wie die indexikalischen kontext-sensitiv sind, fallen die allgemeine Bedeutungsfunktion und die jeweilige Bedeutung zusammen. ${ }^{53}$

Nun ist es zu bemerken, dass für Husserl die allgemeine Bedeutungsfunktion eines

\footnotetext{
${ }^{51}$ Michael Dummett, Origins of Analytical Philosophy, S.47: „It is a great mistake to conclude ... that a word's meaning is in the first instance bestowed on it by an interior act of the individual speaker. It is only from learning language that anyone acquires the very conception of a word's having a meaning. We do not have meanings in our heads waiting for us to attach them to words ... we learn the practice of speaking a language, and learn, in particular, how to form sentences from words and how different words contribute to form sentences that can be used in particular ways ... the fundamental concept is not that of the private meaning-conferring act, but of the social practice of using language."

${ }^{52}$ Unter der Voraussetzung, dass das Identitätskriterium der jeweiligen Bedeutung nicht weniger feinkörnig als das des Gegenstandes sein soll. Mehr darüber vgl. Hua XIX/1, S.430.

${ }^{53} \mathrm{Vgl}$. Christian Beyer, Intentionalität und Referenz, S.72, 75.
} 
Ausdrucks nicht von dem individuellen Sprecher willkürlich, sondern in der Sprachgemeinschaft konventionell (usuell bei Husserl) bestimmt wird. ${ }^{54}$ Was die jeweilige Bedeutung anlangt, so hängt ihre Identität, wie unsere Repräsentation $a(k)$ andeutet, von der Identität der allgemeinen Bedeutungsfunktion und damit der konventionell bestimmten Ebene des Ausdrucks ab. ${ }^{55}$ Um eine Bedeutungsintention, die einem Wortlaut eine von dem Sprecher gewünschte jeweilige Bedeutung verleiht, $\mathrm{zu}$ vollziehen, muss der Sprecher den angemessenen Wortlaut und eventuell (bei indexikalischen Ausdrücken) den angemessenen Äußerungskontext wählen. Diese Angemessenheit ist eben bestimmt durch Konventionen der Sprachgemeinschaft. Der Sprecher muss also die konventionellen Regeln der betreffenden Sprache lernen, um kompetent zu sein, den angemessenen Wortlaut und Kontext für die gewünschte jeweilige Bedeutung auszusuchen. Mit einem anderen Wort, er kann Bedeutungen nicht beliebig verleihen, sondern muss die sozial-konventionelle Dimension der Sprache respektieren. In diesem Sinne ist Dummetts Vorwurf, dass Husserls Begriff von Bedeutungsintention, wie die Humpty-Dumpty-Theorie, die soziale Dimension der Sprache ignoriert, ein Missverständnis, welches darauf beruht, dass Dummett Husserls Unterscheidung zwischen allgemeiner Bedeutungsfunktion und jeweiliger Bedeutung verpasst. $^{56}$

[Kritische Anmerkung: In Husserls Modell über Kommunikation sind zwei bedeutungverleihende Akte zu unterscheiden, nämlich den inneren meinenden Akt, d.i. die Bedeutungsintention (z.B. der Urteilsakt, dass der Himmel blau ist), und den äußeren Sprechakt, der den Wortlaut ausspricht; und nur durch den Vollzug eines angemessenen äußeren Aktes (z.B. durch das Aussagen „,der Himmel ist blau“, aber nicht das Aussagen ,der Himmel ist rot“) lässt sich die betreffende Bedeutung, die durch den inneren Akt (= die Bedeutungsintention) instanziiert wird, dem Ausdruck verleihen. In Husserls Ausführungen über Kommunikation scheint der Sprecher ein ideal-kompetenter Sprecher zu sein, d.i. ein Sprecher, der immer den passenden äußeren

\footnotetext{
${ }^{54}$ Hua XIX/1, S.92: ,[Die wesentlich okkasionellen Ausdrücke orientieren] ihre jeweilige Bedeutung erst nach dem Einzelfall ... während doch die Weise, in der Sie dies tun, eine usuelle ist.“

${ }^{55}$ Christian Beyer, Intentionalität und Referenz, S.72, 79.

${ }^{56}$ Ebd., S.78.
} 
Sprechakt ausübt, um das durch den inneren Akt Gemeinte auszudrücken. Aber Idealisierung führt oft zur Vereinfachung und sogar Übervereinfachung - Husserl hat es unterlassen, den Hintergund des Sprachenlernens in Rücksicht zu nehmen: Niemand ist geboren ein kompetenter Sprecher. Um fähig zu sein, aüßere Sprechakte, die zu inneren Akten passen, systematisch auszuüben, muss das Subjekt die betreffende Sprache schon durch Erziehung und Ausbildung gelernt, erworben und beherrscht haben. Das bloße Zusammensein der äußeren und inneren Akte mag auch bei jemandem, der die betreffende Sprache gar nicht kennt, geschehen: Z.B. kann ein Chinese, der gar kein Deutsch spricht, denken, dass der Himmel blau ist, und zugleich den Wortlaut „,der Himmel ist blau“" (zufällig) produzieren, wollen wir wirklich sagen, dass auch hier das Bedeutungverleihen vollzogen wird? Meines Erachtens übernehmen die äußeren und inneren Akte die Funktion des Bedeutungverleihens nur gegen den Hintergrund vom Sprachenlernen des Sprechers. Dieser Hintergrund bleibt durch Husserls stillschweigende Voraussetzung eines ideal-kompetenten Sprechers leider verdeckt und unbeachtet. $]^{57}$

Kehren wir zu Husserls Begriff von Bedeutungsintention zurück. Im Vollzug der Bedeutungsintention intendiert das Subjekt primär nicht den Wortlaut, sondern den Gegenstand, den der Wortlaut meint. Husserl schreibt, „Das Wort (als äußeres Individuum) ist uns zwar noch anschaulich gegenwärtig, es erscheint noch; aber wir haben es darauf nicht abgesehen, im eigentlichen Sinne ist es jetzt nicht mehr der Gegenstand unserser „psychischen Betätigung“. Unser Interesse, unsere Intention, unser Vermeinen ... geht ausschließlich auf die im sinngebenden Akt gemeinte Sache. “58 Außerdem gewinnt der Wortlaut, der durch die Bedeutungsintention zum Ausdruck wird, auch dieselbe gegenständliche Beziehung - in dem Fall, wo ein angemessener Ausdruck verwendet wird, bezieht er sich auf dieselbe Gegenständlichkeit wie die Bedeutungsintention. ${ }^{59}$

Husserl hält Ausdrucksbedeutungen, wie schon erläutert (im [§3]), für ideale

\footnotetext{
${ }^{57}$ Diese kritische Anmerkung ist inspiriert durch Prof. Felix Mühlhölzers wittgensteinsche Kritiken über die originale Version der vorliegenden Arbeit.

${ }^{58}$ Hua XIX/1, Logische Untersuchungen, S.46-47.

${ }^{59}$ Ebd., S.54.
} 
Spezies. Sie verhalten sich zu Bedeutungsintentionen wie Rot-Spezies zu roten Dingen. Genauer gesagt ist das Rotmoment eines roten Dinges für die Instanziierung der RotSpezies verantwortlich; dasselbe gilt auch für Bedeutungen als Spezies. Welche Momente der Bedeutungsintention sind für die Instanziierung der Bedeutung verantwortlich? Husserls Antwort ist das intentionale Wesen (qua individuelles Moment des Aktes), nämlich die Einheit der individuellen Aktmaterie und Aktqualität: der Urteilsakt „,der Sieger von Jena ist Franzose“ und der Urteilsakt „der Besiegte von Waterloo ist Franzose" haben verschiedenartige intentionale Wesen und somit instanziieren verschiedene Bedeutungen, weil ihre individuellen Materien verschiedenen Spezies (idealen Materien als Spezies) unterstehen, obwohl die Qualität (qua Spezies) der beiden identisch ist; der Zweifelakt „ist der Himmel blau?“ und der Urteilsakt „,der Himmel ist blau“ haben verschiedenartige intentionale Wesen und somit instanziieren verschiedene Bedeutungen, weil ihre individuellen Qualitäten verschiedenen Spezies (idealen Qualitäten als Spezies) unterstehen, obwohl die Materie (qua Spezies) der beiden identisch ist. Durch ideirende Abstraktion (oder kurz: Ideation) ${ }^{60}$ des intentionalen Wesens (qua individuelles Moment) von Bedeutungsintention(en) erfasst man die ideale Bedeutung, ganz wie die Rot-Spezies sich durch Ideation der individuellen Rotmomente von roten Dingen ergibt. Aus diesem Grund bezeichnet Husserl das intentionale Wesen der Bedeutungsintention auch als das bedeutungsmäßige Wesen. ${ }^{61}$

Nach Husserl verleiht die Bedeutungsintention dem Ausdruck nicht nur eine Bedeutung überhaupt, sondern eine bestimmte Bedeutung: die verliehene Bedeutung ist eben diejenige Bedeutung, die sich durch Ideation des bedeutungsmäßigen Wesens der Bedeutungsintention ergibt. Der englische Ausdruck ,the sky is blue“ hat die Bedeutung ,der Himmel ist blau“ deswegen, weil das bedeutungsmäßige Wesen der Bedeutungsintention, die ein kompetenter Sprecher von Englisch vollzieht, diese

\footnotetext{
${ }^{60}$ Grob gesagt ist die Ideation ein Verfahren, in dem man durch Vergleichung und Abstraktion von Einzelheiten (z.B. rote Dinge) die gemeinsame Spezies (die Spezies Rot) von ihnen erfasst. Eine typische Anwendung des Ideationsverfahrens in Logischen Untersuchungen ist, dass Husserl durch Ideation die Intentionalität als gemeinsamen Charakter von allen Bewußtseinsakten erfasst (V.LU, §10). Darüber vgl. auch Christian Beyer, Von Bolzano zu Husserl. Eine Untersuchung über den Ursprung der phänomenologischen Bedeutungslehre, Kluwer Academic Publishers, Dordrecht/Boston/London 1996, S.153ff.

${ }^{61}$ Hua XIX/1: Logische Untersuchungen, S.431.
} 
Bedeutung instanziiert.

Wir können das Obige wie folgt zusammenfassen: (a) ein bloßer Wortlaut wird dadurch zum Ausdruck, dass eine Bedeutungsintention ihm eine bestimmte Bedeutung verleiht; (b) die verliehene Bedeutung ist eben die Bedeutung, die durch das intentionale Wesen der Bedeutungsintention instanziiert wird.

Neben dem Verhältnis des Bedeutungverleihens besteht in der Kommunikation noch ein anderes Verhältnis von Bedeutungsintention und Ausdruck, nämlich das Verhältnis der Kundgabe. ${ }^{62}$ Eine erfolgreiche Kommunikation erfordert Kooperation von Sprecher und Hörer: Einerseits erzeugt der Sprecher als Teilnehmer der Kommunikation mündlich oder schriftlich den Wortlaut, und natürlich vollzieht er zugleich die entsprechende Bedeutungsintention, z.B. einen Urteilsakt; andererseits muss der Hörer seine eigene Bedeutungsintention vollziehen, nämlich den Wortlaut verstehen, gleichgültig, ob er den intendierten Sachverhalt für wahr hält oder nicht; und der Hörer muss noch den Wortlaut als einen vom Sprecher sinnbelebten Ausdruck (und keinen bloß physischen Gegenstand) auffassen - der Wortlaut dient dem Hörer als ein Zeichen dafür, dass der Sprecher eine dem Ausdruck entsprechende Bedeutungsintention (z.B. einen Urteilsakt) vollzieht und dem Hörer die dabei verliehene Bedeutung mitteilen will. In Husserls Terminologie wird die Bedeutungsintention und die mitteilende Absicht in dieser Kooperation durch den Ausdruck kundgegeben. ${ }^{63}$

Genauer gesprochen fasst Husserl den Begriff von Kundgabe in zwei Sinnen. Im engeren Sinne wird nur die Bedeutungsintention durch den Ausdruck kundgegeben, während im weiteren Sinne auch andere psychische Erlebnisse (z.B. die mitteilende Absicht, eine Wahrnehmung, ein Wunsch ${ }^{64}$ ) kundgegeben werden.

Ein Sprecher kann den Aussagesatz „der Himmel ist blau“ aussprechen, ohne jede Anschauung des blauen Himmels zu haben, dann vollzieht er eine leere Bedeutungsintention. Aber es gibt auch Fälle, wo sich die Bedeutungsintention mit

\footnotetext{
62 Ebd., S.39-41.

${ }^{63}$ Ebd., S.40: „[Ausdrücke] dienen dem Hörenden als Zeichen für die „Gedanken“ des Redenden, d.h. für die sinngebenden psychischen Erlebnisse desselben, sowie für die sonstigen psychischen Erlebnisse, welche zur mitteilenden Intention gehören. Diese Funktion der sprachlichen Ausdrücke nennen wir die kundgebende Funktion. “ ${ }^{64}$ Z.B. wenn der Sprecher zu dem Hörer sagt, „Ich sehe einen Baum“ oder „Ich wünsche dir eine angenehme Reise“.
} 
einer Anschauung (Wahrnehmung, Erinnerung, Phantasie usw.) derselben Gegenständlichkeit deckt. In solchen Fällen ist die Bedeutungsintention durch die Anschauung ,erfüllt“. Wenn der Sprecher den blauen Himmel wahrnimmt und aufgrund dieser Wahrnehmung den Aussagesatz „der Himmel ist blau“ behauptet, dann befinden sich die Bedeutungsintention und die Anschauung in einer identifizierenden oder positiven Erfüllungseinheit (oder Erkenntniseinheit): der Gegenstand der Bedeutungsintention indentifiziert sich mit dem der Anschauung. ${ }^{65}$ In manchen Fällen findet die Bedeutungsintention (z.B. das Urteil „der Himmel ist blau“) in der Anschauung (z.B. die Wahrnehmung, dass der Himmel grau ist) eine negative Erfüllung oder „Enttäuschung“, dann befinden sich die beiden Akte in einer unterscheidenden bzw. negativen Erfüllungseinheit. ${ }^{66}$ Die Bedeutungsintention, die isoliert und somit leer sein kann, findet in der Erfüllungseinheit ihre positive oder negative Erfüllung. In diesem Sinne nennt Husserl die gelegentlich stattfindende Anschauung, die sich mit der Bedeutungsintention $\mathrm{zu}$ einer Erfüllungseinheit zusammenschließt, bedeutungerfüllenden Akt oder Bedeutungserfüllung. ${ }^{67}$

In der VI. $L U$ vertritt Husserl die These, dass objektivierende Akte genau diejenigen Akte sind, die die Bedeutungsfunktion im weiteren Sinne (= die bedeutungverleihende bzw. bedeutungerfüllende Funktion) ${ }^{68}$ übernehmen und somit direkt in eine Erfüllungseinheit treten können. ${ }^{69}$ Die objektivierenden Akte, die die bedeutungverleihende Funktion übernehmen und somit als Bedeutungsintention fungieren können, sind in Husserls Terminologie signitiv ${ }^{70}$; die Akte, die die

\footnotetext{
${ }^{65}$ Hua XIX/2: Logische Untersuchungen. Zweiter Band. Zweiter Teil. Untersuchungen zur Phänomenologie und Theorie der Erkenntnis, hrsg. von U. Panzer, The Hague/Boston/Lancaster: Martinus Nijhoff 1984, S.44, 556, 566568, 584, 596-597.

${ }^{66}$ Nach Husserl ist Enttäuschung keine bloße Privation der (positiven) Erfüllung, sondern eine eigenartige Einheit von der Bedeutungsintention und der enttäuschenden Anschauung (,,eine so eigenartige Form der Synthesis wie die Erfüllung“). Diese Einheit konstituiert die „Nichtübereinstimmung“ zwischen den Gegenständen der BedeutungsIntention und -Erfüllung. Vgl. ebd., S.574-576.

${ }^{67}$ Hua XIX/1: Logische Untersuchungen, S.44.

${ }^{68}$ Hua XIX/2: Logische Untersuchungen, S.549.

${ }^{69}$ Husserl gesteht, dass ein nicht-objektivierender Akt in gewissem Sinn auch erfüllbar ist, aber was in die oben charakterisierte Erfüllungseinheit tritt, ist eigentlich sein fundierend-objektivierender Akt. Z.B. findet die Erfüllung eines Wunsches in zwei Schritten statt: zuerst tritt der fundierend-objektivierende Akt des Wunsches (z.B. eine signitive Vorstellung) direkt in Erfüllungseinheit mit einer Wahrnehmung des gewünschten Gegenstandes, dann wird der Wunsch durch das Stattfinden dieser Erfüllungseinheit erfüllt. Vgl. ebd., S.582-585.

${ }^{70}$ In der VI. $L U$ erwähnt Husserl die Frage, ob signitive Akte auch außerhalb der Bedeutungsfunktion auftreten können (Hua XIX/2, 586) und ob es so etwas wie wortlose signitive Intentionen gibt (a.a.O., 594). Darauf gibt er im [§15] der VI. $L U$ eine bejahende Antwort. Aber dabei konzentriert er sich vor allem auf die Partialintention innerhalb eines anschaulichen Aktes, die auf die nicht-intuitiv gegebene Seite des Gegenstandes gerichtet und von ihm auch
} 
bedeutungerfüllende Funktion übernehmen und somit als Bedeutungserfüllung fungieren können, sind intuitiv. ${ }^{71}$

Im [§4] haben wir objektivierende Akte in nominale und propositionale klassifiziert. Da alle signitiven Akte objektivierende Akte sind, liegt es nahe, die Bedeutungsintentionen in nominale und propositionale einzuteilen. Diese Einteilung ist wichtig für unsere Diskussion über selbständige und unselbständige Bedeutungen im $[\S 8]$.

Zum Schluss können wir drei Sinne von Ausgedrücktsein eines Aktes unterscheiden:

(i) Der Wortlaut drückt die Bedeutungsintention aus, das heißt, er trägt die Bedeutung, die durch die Bedeutungsintention instanziiert wird.

(ii) In der Kommunikation drückt der Wortlaut die Bedeutungsintention noch in einem anderen Sinne aus, das heißt, der Wortlaut fungiert dann als ein Zeichen dafür, dass der Sprecher die entsprechende Bedeutungsintention vollzieht. In Husserls Terminologie ist Ausdrücken in diesem Sinne als Kundgabe (im engeren Sinne) bezeichnet.

(iii) Der bedeutungerfüllende Akt, der sich gelegentlich mit der Bedeutungsintention zu einer Erfüllungseinheit zusammenschließt, ist in einem dritten Sinne durch den Wortlaut ausgedrückt - die Bedeutungsintention (z.B. ein Urteilsakt) und das Erzeugen des Wortlauts sind vollzogen aufgrund der Bedeutungserfüllung (z.B. einer Wahrnehmung). Das Ausgedrücktsein des bedeutungerfüllenden Aktes heißt, dass sie die Bedeutungsintention und das Erzeugen des Wortlauts erkenntnismäßig motiviert. Das heißt, ,daß ich die betreffende Tatsache nicht nur behaupte, sondern wahrnehme und sie so behaupte, wie ich sie wahrnehme. ${ }^{\text {"72 }}$ (Z.B. ich sehe eine rote Kugel und aufgrund dieser Kugel-Wahrnehmung behaupte, „diese Kugel ist rot.“) Es ist zu

als signitiv bezeichnet ist (Hua XIX/2, 589, 594, 623). Rudolf Bernet kritisiert, dass solche leeren Partialintentionen innerhalb der Anschauung keineswegs für signitiv gehalten werden sollen; denn der Unterschied zwischen den leeren Partialintentionen von Anschauung und den leeren Bedeutungsintentionen ist ,,so groß, dass man sich die Frage stellen muss, ob es sich denn wirklich in beiden Fällen um die selbe Form der Leere handelt.“ Mehr darüber vgl. Rudolf Bernet, ,„Intention und Erfüllung, Evidenz und Wahrheit“, in: Edmund Husserl. Logische Untersuchungen, S.197-198.

${ }^{71}$ Hua XIX/2: Logische Untersuchungen, S.567, 586.

${ }^{72}$ Ebd., S.548. 
beachten, dass beim Ausgedrücktsein des bedeutungerfüllenden Aktes der Wortlaut und die ihm zugrundeliegende Bedeutungsintention sich nicht auf den erfüllenden Akt selbst, sondern auf seinen Gegenstand beziehen. ${ }^{73}$

\section{§6. Vorbereitende Analysen V: Husserls Mereologie}

In der III. $L U$ unterscheidet Husserl zwischen selbständigen und unselbständigen Gegenständen (Gegenstände im weitesten Sinne, inklusive individuelle Dinge, individuelle Momente des Dinges, Spezies, Ereignisse usw.). Diese Unterscheidung gilt sowohl für reale als auch für ideale Gegenstände. Selbständig sind diejenigen Gegenstände, die existieren können, ohne mit anderen Gegenständen verbunden zu werden. Z.B. kann ein Ziegel selbständig existieren, ohne ein Bestandstück eines Hauses oder mit anderen Bestandstücken des Hauses verbunden zu sein. Im Vergleich dazu sind diejenigen Gegenstände unselbständig, die nur in Verbindung mit anderen Gegenständen existieren können(Hua XIX/1, 244). Husserls Standardbeispiel für unselbständige Gegenstände sind Ausdehnung und Farbe in der Wahrnehmungssphäre. Es gibt keine Farbe ohne Ausdehnung und umgekehrt, in diesem Sinn sind die beiden voneinander gegenseitig abhängig. Es ist hier $\mathrm{zu}$ betonen, dass die fragliche Unselbständigkeit für Husserl eine apriorische Unselbständigkeit ist und apriorischen Gesetzen unterliegt. Im Vergleich dazu spricht man auch von empirischer Unselbständigkeit, die empirisch-kausalen Gesetzen gehorcht. Man spricht oft davon, dass das Ereignis „die Sonne scheint“ mit dem Ereignis „der Stein wärmt sich" notwendig verbunden ist; diese Notwendigkeit gründet sich auf empirisch-kausale Gesetze. Doch eine gegensätzliche Verbindung ist denkbar: Der Stein wird kälter, weil Sonne scheint. In diesem Sinne ist ein aus empirischen Gesetzen stammende Notwendigkeit nur eine Tatsächlichkeit - man kann sich eine mögliche Welt ausdenken, deren empirisch-kausale Gesetze ganz anders aussehen als die der wirklichen Welt. (a.a.O., 242-244)

Im Gegensatz dazu ist die Unabhängigkeit oder Selbständigkeit der Farbe von

\footnotetext{
${ }^{73}$ Ebd.:,,Nicht über die Wahrnehmung, sondern über das Wahrgenommene wird hierbei das Urteil gefällt.“
} 
einer Ausdehnung undenkbar. Es handelt sich bei solcher Undenkbarkeit, so betont Husserl, nicht um eine subjektive Unfähigkeit, etwas auszudenken, sondern sie gründet sich auf das Wesen der Gegenstände. ${ }^{74}$ Das ist eine ganz andere Lesart der Undenkbarkeit als die Lesart von Schlick. ${ }^{75}$ Positiv gesprochen schreibt das Wesen des Gegenstandes ein apriorisches Gesetz vor, dass ein Gegenstand x, der dem Wesen (bzw. Gattung) X (z.B Farbe) untersteht, nur in Verbindung mit einem Gegenstand y, der dem Wesen Y (z.B. Ausdehnung) untersteht, innerhalb eines Ganzen der Sorte Z (ausgedehnte Farbe) existieren kann (a.a.O., 244). Aufgrund dieses Begriffs von Unselbständigkeit entwickelt Husserl den Begriff der Fundierung: Wenn ein dem Wesen X unterstehender Gegenstand x nur dann existieren kann, wenn er mit einem dem Wesen $\mathrm{Y}$ unterstehenden Gegenstand y verbunden ist, dann sagen wir, $\mathrm{x}$ ist fundiert in $\mathrm{y}^{76}$

Die Unterscheidung zwischen selbständigen und unselbständigen Gegenständen, wie gesagt, gilt nicht nur für reale Gegenstände, sondern auch für ideale Gegenstände und besonders für Bedeutungen. ${ }^{77}$ Deshalb können wir innerhalb der Bedeutungssphäre

\footnotetext{
${ }^{74}$ Hua XIX/1, S.242: „Unterschied wie dieser, daß ein Gegenstand ... an und für sich sein kann, ein anderer nur in oder an einem anderen sein kann, betreffen nicht Faktizität unseres subjektiven Denkens. Es sind sachliche, im reinen Wesen der Sachen gründende Unterschiede, die aber, weil sie bestehen und wir von ihnen wissen, uns zur Aussage bestimmen: es sei ein davon abweichendes Denken unmöglich, d.h. ein davon abweichendes Urteilen sei verkehrt. Was wir nicht denken können, kann nicht sein, was nicht sein kann, können wir nicht denken...“

${ }^{75}$ Nach Schlicks Wittgenstein-inspirierter Konzeption besteht die apriorische Natur von Sätzen wie ,Jede Farbe hat Ausdehnung“ nur darin, dass ,,in ihnen zeigt sich ... nur die Art und Weise, wie wir unsere Worte verwenden.“ Für Schlick drücken diese bei Husserl material-aprirosche Sätze eigentlich bloß linguistische Tatsachen aus. Dabei geht es gar nicht um irgenein gesetzmäßiges „Sosein“ der Wirklichkeit, wie Husserl es denkt. Darauf kann man in Husserls Geist erwidern: Ja. Vielleicht drücken diese Sätze Verwendungsregeln der Worte aus. Aber man kann weiter fragen, warum sind die Regeln so und nicht anders? Verschiedene Sprachen folgen denselben Regeln der Farb- und Ausdehnungsworte, würde das nicht eine merkwürdige Zufälligkeit sein, wenn solche Regeln bloß linguistischkonventionell wären? Eine mögliche Erklärung dafür ist, dass der Grund für diese Regeln eben im sachlichen „Sosein“ der Welt besteht. Schlicks linguistische Lesart über die Undenkbarkeit schließt also Husserls ontologische Lesart nicht ohne weiteres aus. Allerdings hat Husserls Lesart andere Schwierigkeiten: Garantiert jede Undenkbarkeit ein ontologisches, und sogar notwendiges „Sosein“ der betreffenden Gegenstände? Husserls Antwort ist nein, denn es gibt auch Undenkbarkeit wegen der Beschränkung des menschlichen Denkens. Aber was ist das Kriterium, wonach wir die ,sachliche“ Undenkbarkeit und die „menschliche“ Undenkbarkeit unterscheiden? Dazu sagt Husserl leider nicht. Andererseits scheint Denkbarkeit/Undenkbarkeit kontext-relativ zu sein: In alltäglichen Kontext hat man Recht, zu sagen, jede Farbe habe Ausdehnung, aber in wissenschaftlichen Kontexten, wo Farben als Lichtwelle mit diesen und jenen Eigenschaften konzipiert werden (und ähnlich bei Ausdehnung), dann muss auch das Verhältnis zwischen Farbe und Ausdehnung anders konzipiert werden (Hat Lichtwelle Ausdehnung?). Das Wesentliche mag mit Kontexten variieren. Davon spricht Husserl auch nicht.

${ }^{76}$ Ebd., S.267ff. Thomas Nenon weist darauf hin, dass Husserls Fundierungsbegriff zweideutig ist und durch zwei Modelle verstanden werden kann. Nach dem „ontologischen Modell“ ist ein unselbständiger Gegenstand auf einem komplizierten, ihn enthaltenden Gegenstand fundiert - das Ganze hat hier die grundlegende Priorität und spielt die fundierende Rolle; das ,epistemologische Modell“ verwertet das architektonische Bild und betont die Funktion gewisser fundamentaler Teile eines komplizierten Ganzen - solche Teile haben hier die grundlegende Priorität und spielen die fundierende Rolle. Vgl. Thomas Nenon, ,Two Models of Foundation in the Logical Investigations“, in: Husserl in Contemporary Context, edited by Burt C. Hopkins, Springer Science + Business Media, Dordrecht 1997 , S.97ff.

${ }^{77}$ Hua XIX/1: Logische Untersuchungen, S.301, 318-321.
} 
die Unterscheidung von selbständigen und unselbständigen Bedeutungen machen und die Verbindungsgesetze der Bedeutungen studieren. Das ist eben das Thema der IV. LU, die dementsprechend als „Der Unterschied der selbständigen und unselbständigen Bedeutungen und die Idee der reinen Grammatik“ betitelt wird. Darüber diskutieren wir im folgenden Kapitel. 


\section{KAPITEL}

\section{REINE GRAMMATIK UND EMPIRISCHE GRAMMATIK}

\section{§7. Kategorematische und synkategorematische Ausdrücke}

Nehmen wir den Ausgang von dem Unterschied zwischen einfachen und zusammengesetzten Bedeutungen. ${ }^{78}$ Husserls Beispiele für zusammengesetzte Bedeutungen sind „ein Mann wie von Eisen“, „,ein König, der die Liebe seiner Untertanen erwirbt" usw. Solche Bedeutungen bestehen aus Teilbedeutungen wie „Mann“, „Eisen“, „König“, „Liebe“. Die Teilbedeutungen einer zusammengesetzten Bedeutung könnten auch zusammengesetzte Bedeutungen sein und sich wieder in Teilbedeutungen zerlegen; allerdings kann dies nicht in infinitum fortgehen. Husserl zufolge wird man in der fortsetzenden Teilung schließlich auf einfache Bedeutungen stoßen, nämlich Bedeutungen, die nicht mehr Teilbedeutungen als Komponenten enthalten. Husserls Beispiele für einfache Bedeutungen sind Eigenbedeutungen (Bedeutungen von Eigennamen) $)^{79}$.

Demsprechend gibt es auf der Ausdrucksebene zusammengesetzte und einfache Ausdrücke: Zusammengesetzte Ausdrücke sind Ausdrücke, deren Bedeutungen zusammengesetzt sind; einfache Ausdrücke sind Ausdrücke, deren Bedeutungen einfach sind (Hua XIX/1, 319).

Ein zusammengesetzter Ausdruck ist in der Regel eine Wortkomplexion, die in sich mehrere Teilausdrücke (Worte oder Morpheme) enthält. Hierbei stellt sich die Frage, ob jedem Teil des zusammengesetzten Ausdrucks eine eigene Bedeutung zuzuordnen ist (a.a.O., 310-311).

Wir können die Frage noch genauer formulieren. Grammatiker machen die Unterscheidung zwischen kategorematischen und synkategorematischen Ausdrücken (a.a.O.). Diese Unterscheidung ist von Husserl anerkannt und revidiert. Husserl zufolge ist ein Ausdruck kategorematisch, wenn er für sich eine Bedeutungsintention

\footnotetext{
${ }^{78}$ Hua XIX/1: Logische Untersuchungen, S.303.

${ }^{79}$ Ebd., 306.
} 
kundgeben kann. Z.B. sind Ausdrücke wie „,der König“ (Namen), „,der Himmel ist blau“ (Aussagesätze) kategorematisch. ${ }^{80} \mathrm{Im}$ Vergleich dazu ist ein synkategorematischer Ausdruck nicht in der Lage, eine Bedeutungsintention alleine kundzugeben. Er muss sich zuerst mit anderen Ausdrücken zu einem umfassenderen kategorematischen Ausdruck zusammenschließen, um an der Kundgabe einer Bedeutungsintention beteiligt zu werden. Beispiele für synkategorematische Ausdrücke sind ,und“, „oder“, ,geht“, „,des Vaters“ usw.(a.a.O., 310-313) Diese Unterscheidung verhilft uns zu einer neuen Formulierung der obigen Frage: Haben synkategorematische Ausdrücke ihre eigene Bedeutung? Dabei gehen die Meinungen auseinander.

Einerseits stehen manche Philosophen (wie Bolzano) auf dem Standpunkt, dass jeder Ausdruck, ob kategorematisch oder synkategorematisch, seine eigene Bedeutung hat, und dass er zu der Gesamtbedeutung eines zusammengesetzten Ausdrucks seine eigene Bedeutung als Teilbedeutung beiträgt, wenn er mit anderen Ausdrücken zu einem zusammengesetzten Ausdruck kombiniert wird.

Andererseits sind manche (wie Marty) ${ }^{81}$ der Auffassung, dass ein synkategorematischer Ausdruck (z.B. ,„und“) für sich bedeutungslos ist, und dass er in einer umfassenderen Wortkomplexion (z.B. „,Sokrates und Platon“) bloß dabei hilft, einen kategorematischen Ausdruck, der seine eigene Bedeutung hat, aufzubauen. Wenn herausgerissen, hat ein synkategorematischer Ausdruck gar keine Bedeutung. ${ }^{82}$ Nach der von Husserl revidierten Version von Martys Auffassung sind nur zwei Ausdrucksarten kategorematisch, nämlich Namen und Sätze; alle anderen Ausdrucksarten sind synkategorematisch (a.a.O., 312). Mit Martys eigenen Worten: Kategorematsiche Ausdrücke sind selbstbedeutend, weil sie ihre jeweilige eigene

\footnotetext{
${ }^{80}$ Für die Grammatiker sind neben Namen und Aussagesätzen andere Satztypen wie Fragesätze, Wunschsätze auch kategorematisch. Doch für Husserl lassen sich diese anderen Satztypen auf Namen oder Aussagesätze reduzieren. Denn Husserls Meinung nach ist der Akt, der dem Wunschsatz „Gott stehe mir bei“ Bedeutung verleiht, nicht der Wunsch selbst, sondern ein Akt, der den Wunsch nennt oder über ihn urteilt. Deshalb ist hier wie im Folgenden die Betrachtung der kategorematischen Ausdrücke nur auf Namen und Aussagesätze eingeschränkt. Vgl. ebd., S.311 312.

${ }^{81}$ Ebd., S.312.

${ }^{82}$ Husserls Zusammenfassung dieser von Marty inspirierten Auffassung lautet: , ... da der Unterschied zwischen Kategorematischem und Synkategorematischem ein grammatischer ist, so möchte es scheinen, daß auch die Sachlage, die ihm zugrunde liegt, eine „,bloß grammatische“ sei. Wir bedienen uns des öfteren mehrer Worte, um eine „Vorstellung“ auszudrücken - das liegt, könnte man denken, an zufälligen Eigenheiten der jeweiligen Sprache. Die Gliederungen im Ausdruck ist ohne alle Beziehung zu irgendwelchen Gliederungen in der Bedeutung. Die synkategorematischen Worte, die ihn aufbauen helfen, sind also eigentlich ganz bedeutungslos, und nur dem gesamten Ausdruck kommt wahrhaft eine Bedeutung zu." Ebd.
} 
Bedeutung haben; synkategorematische Ausdrücke sind mitbedeutend, weil sie für sich keine Bedeutung besitzen. ${ }^{83}$ Demnach hätte Husserl synkategorematische Ausdrücke als synkategorematische Zeichen bezeichnen sollen, denn für ihn hat ein Ausdruck immer seine Bedeutung.

Husserl steht Bolzano näher als Marty: Er will annehmen, dass ein synkategorematischer Ausdruck seine eigene Bedeutung hat und in einem umfassenderen Ausdruckszusammenhang seine eigene Bedeutung $\mathrm{zu}$ der Gesamtbedeutung beiträgt. Deshalb darf man den Unterschied zwischen kategorematischen und synkategorematischen Ausdrücken nicht auf den Unterschied zwischen selbstbedeutenden Ausdrücken und mitbedeutenden Zeichen zurückführen. ${ }^{84}$

Allerdings glaubt Husserl, dass Martys Versuch, den Unterschied von kategorematischen/synkategorematischen Ausdrücken auf ihre jeweiligen bedeutungsmäßigen Eigenheiten zurückzuführen, ,sicherlich irgendeine Berechtigung hat"(a.a.O.). Husserl muss also einen anderen Weg suchen, die bedeutungsmäßigen Eigenheiten von kategorematischen und synkategorematischen Audrücken zu charakterisieren.

\section{§. Selbständige und unselbständige Bedeutung}

Husserls Art und Weise, den bedeutungsmäßigen Unterschied zwischen kategorematischen und synkategorematischen Ausdrücken zu charakterisieren, hängt mit seiner intentionalitätstheoretischen Auffassung der Bedeutung ${ }^{85}$ zusammen. Diese intentionalitätstheoretische Auffassung der Bedeutung enthält drei Thesen:

(1) Eine Bedeutungsintention instanziiert eine Bedeutung als ideale Einheit, oder umgekehrt, die ideale Bedeutung, die durch die Bedeutungsintention instanziiert wird,

\footnotetext{
${ }^{83}$ Vgl. Jocelyn Benoist, „,Grammatik und Intentionalität“, in: Edmund Husserl. Logische Untersuchungen, S.127. ${ }^{84}$ Die Bolzano-Husserl-Auffassung entspricht etwa Freges Bild über sprachliche Bedeutungen:,,Die Leistungen der Sprache sind wunderbar. Mittels weniger Laute und Lautverbindungen ist sie imstande, ungeheuer viele Gedanken auszudrücken und zwar auch solche, die noch nie vorher von einem Menschen gefasst und ausgedrückt worden sind. Wodurch werden diese Leistungen möglich? Dadurch, dass die Gedanken aus Gedankenbausteinen aufgebaut werden. Und diese Bausteine entsprechen Lautgruppen, aus denen der Satz aufgebaut wird, der den Gedanken ausdrückt, sodass dem Aufbau des Satzes aus Satzteilen der Aufbau des Gedankens aus Gedankenteilen entspricht.“"(Frege, 1969, S.243.)

${ }^{85}$ Oder, mit Tugendhat zu sagen, ein gegenstandstheoretischer Ansatz der Bedeutungstheorie. (Tugendhat 1976, 8.10. Vorlesungen)
} 
entspricht der Bedeutungsintention;

(2) Eine Bedeutungsintention ist ein intentionales Erlebnis, und als intentionales Erlebnis hat sie Intentionalität - sie intendiert eine Gegenständlichkeit;

(3) Die Bedeutung, die als ideale Spezies einer Bedeutungsintention entspricht, intendiert dieselbe Gegenständlichkeit wie die Bedeutungsintention und zwar in derselben durch die Materie bestimmten Weise ${ }^{86}$. Sie teilt dieselbe Intentionalität mit dem Akt.

Bei These (1) handelt es sich um das Verhältnis zwischen Bedeutungsintention und ihrer entsprechenden Bedeutung: Jede Bedeutungsintention instanziiert eine Bedeutung; oder umgekehrt, die Bedeutung als ideale Spezies entspricht der Bedeutungsintention ${ }^{87}$ als einer ihr unterstehenden Einzelheit.

Husserl zufolge können Bedeutungsintentionen auch einfach oder zusammengesetzt sein. ${ }^{88}$ Z.B. ist die die Eigenbedeutung „Sokrates“ instanziierende Bedeutungsintention einfach. Aber sie kann sich mit einer anderen Bedeutungsintention, die die Bedeutung „Platon“ instanziiert, durch die konjunktionale Verbindungsform „und“ zu einer umfassenderen Bedeutungsintention, der die zusammengesetzte Bedeutung „Sokrates und Platon“ entspricht, zusammensetzen; die umfassendere Bedeutungsintention ist eine zusammengesetzte Bedeutungsintention. ${ }^{89}$

Im obigen Beispiel entspricht weder die Bedeutung „Sokrates“, noch die Bedeutung „und“, noch die Bedeutung „Platon“ dem zusammengesetzten Bedeutungsintention „Sokrates und Platon“, sondern sie entsprechen jeweils einem Teilakt von ihr. Doch die Teilakte, denen die Bedeutungen ,SSokrates“ und „Platon“ jeweils entsprechen, sind selbständige Teile und können selber als vollständige Bedeutungsintention fungieren, während der Teilakt, dem die Bedeutung „und“ entspricht, nur ein unselbständiger Teil des Gesamtaktes ist und niemals als vollständige Bedeutungsintention fungieren kann. Dabei haben wir

\footnotetext{
${ }^{86} \mathrm{Vgl}$. oben [\$4].

${ }^{87}$ Genauer: ihrem bedeutungsmäßigen Wesen (als Aktmoment) oder Materie (als Moment). Dazu vgl. auch [§43bc] der vorliegenden Arbeit.

${ }^{88}$ Hua XIX/1: Logische Untersuchungen, S.320.

${ }^{89}$ Damit hängt der von Beyer inspirierte Begriff der strukturellen Isomorphie zwischen Bedeutungen und Bedeutungsintentionen zusammen, wovon später gesprochen wird. Vgl. [§47] der vorliegenden Arbeit.
} 
zwei Bedeutungsarten konstatiert: Bedeutungen, die einer vollständigen Bedeutungsintention entsprechen können, und Bedeutungen, die es nicht können. ${ }^{90}$ Wir können die ersteren als selbständige Bedeutungen, und die letzteren als unselbständige Bedeutungen bezeichnen:

\section{(Charakterisierung von selbständiger und unselbständiger Bedeutung)}

Eine Bedeutung ist selbständig, wenn sie einer vollständigen

Bedeutungsintention entsprechen kann; sie ist unselbständig, wenn sie es nicht kann.

Bedeutungsintentionen sind objektivierende Akte. Im [§4] haben wir festgestellt, dass objektivierende Akte entweder nominal oder propositional ist. Daher ist eine (vollständige) Bedeutungsintention entweder nominal oder propositional. Dementsprechend ist die Bedeutung, die einer vollständigen Bedeutungsintention entspricht und dem kundgebenden Ausdruck verliehen wird, entweder nominal oder propositional. Mit anderen Worten, nur nominale oder propositionale Bedeutungen können einer vollständigen Bedeutungsintention entsprechen und somit in die Bedeutungsart selbständige Bedeutung eingeordnet werden. Bedeutungen anderer Arten, z.B. konjunktive Bedeutungen, sind unselbständig.

Husserl beschreibt die bedeutungsmäßigen Eigenheiten von kategorematischen und synkategorematischen Ausdrücken durch die Unterscheidung zwischen selbständigen und unselbständigen Bedeutungen: Ein synkategorematischer Ausdruck hat doch seine eigene Bedeutung, aber seine Bedeutung ist unselbständig, während die Bedeutung eines kategorematischen Ausdrucks selbständig ist. ${ }^{91}$ Das Monopol, das Namen und Aussagesätze auf kategorematische Ausdrücke haben, besteht darin, dass nur sie als Träger der einzig beiden selbständigen Bedeutungsarten (nominale und

\footnotetext{
${ }^{90}$ Hua XIX/1, S.320-321: „,Demgemäß werden wir eine Bedeutung selbständig nennen, wenn sie die volle und ganze Bedeutung eines konkreten Bedeutungsaktes ausmachen kann, und unselbständig, wenn dies nicht der Fall ist. Sie kann dann nur in einem unselbständigen Teilakt eines konkreten Bedeutungsaktes realisiert sein, nur in Verknüpfung mit gewissen anderen, sie ergänzenden Bedeutungen kann sie Konkretion gewinnen, nur in einem Bedeutungsganzen kann sie „sein“. Die so definierte Unselbständigkeit der Bedeutung als Bedeutung bestimmt nach unserer Auffassung das Wesen der Synkategorematika.“

${ }^{91}$ Ebd., S.318: „Wir haben erkannt, daß der scheinbar so gleichgültigen Unterscheidung der Ausdrücke in kategorematische und synkategorematische eine fundamentale Scheidung im Gebiete der Bedeutungen entspricht. Hatten wir auch die erstere zum Ausgangspunkt genommen, so zeigte sich doch die letztere als die ursprüngliche, nämlich als die jene grammatische Unterscheidung allererst begründende.“
} 
propositionale Bedeutung) fungieren.

Thesen (2) und (3) zufolge übernimmt eine selbständige Bedeutung die Intentionalität der Bedeutungsintention, der sie entspricht. Es verhält sich ganz anders bei einer unselbständigen Bedeutung. Ein Akt(-teil), dem eine unselbständige Bedeutung entspricht, hat keine Intentionlität und natürlich kann er keine Intentiontalität auf die entsprechende unselbständige Bedeutung übertragen. Ein solcher Akt(-teil) (z.B. der, dem die Bedeutung ,,und“ entspricht) kann nur in einer umfassenderen Bedeutungsintention (z.B. ein Akt, dem die Bedeutung ,Sokrates und Platon“ entspricht) als Bestandteil fungieren. Wir können die Unterscheidung von selbständigen und unselbständigen Bedeutungen auch wie folgt charakterisieren:

(Charakterisierung* von selbständiger und unselbständiger Bedeutung)

Eine Bedeutung ist selbständig, wenn sie dieselbe Intentionalität von der sie instanziierenden Bedeutungsintention übernehmen kann; oder unselbständig, wenn sie es nicht kann.

(Charakterisierung*) ist äquivalent mit (Charakterisierung), denn eine Bedeutung kann die Intentionalität von der sie instanziierenden Bedeutungsintention übernehmen, genau dann, wenn sie einer vollständigen Bedeutungsintention entsprechen kann.

\section{§9. Intentionlitäts-Abhängigkeit/-Unabhängigkeit von Bedeutungen}

Husserl hält seine Unterscheidung von selbständigen und unselbständigen Bedeutungen als Anwendung der allgemeineren Unterscheidung von selbständigen und unselbständigen Gegenständen auf die Bedeutungssphäre. ${ }^{92}$ Genauer gesehen ist die Anwendung nicht so direkt wie Husserl dachte. Bei der allgemeinen Unterscheidung von selbständigen/unselbständigen Gegenständen, die in III. $L U$ entwickelt wurde, handelt es sich um die Existenz-Unabhängigkeit/-Abhängigkeit der Gegenstände; bei der Unterscheidung von selbständigen/unselbständigen Bedeutungen handelt es sich, genauer gesagt, um die Intentionalitäts-Abhängigkeit/-Unabhängigkeit der Bedeutungen.

\footnotetext{
${ }^{92}$ Hua XIX/1, Logische Untersuchungen, S.301.
} 
Husserl zufolge hat jeder Ausdruck seine eigene Bedeutung, gleichgültig, ob er kategorematisch oder synkategorematisch ist. Und ein synkategorematischer Ausdruck behält noch seine eigene Bedeutung, auch wenn er isoliert betrachtet wird. ${ }^{93}$ Die Existenz einer unselbständigen Bedeutung ist also nicht von der Existenz anderer Bedeutungen, die mit ihr zu einer kategorematischen Bedeutung kombiniert werden, abhängig. Daher kann die Unselbständigkeit/Selbständigkeit einer Bedeutung nicht durch ihre Existenz-Abhängigkeit/-Unabhängigkeit erklärt werden.

Erinnern wir uns an Husserls eigene Definition der Selbständigkeit/Unselbständigkeit von Bedeutungen: Eine Bedeutung ist selbständig, wenn sie einer vollständigen (selbständigen) Bedeutungsintention entsprechen kann; sie ist unselbständig, wenn sie es nicht kann. ${ }^{94}$ Dabei wird die Selbständigkeit/Unselbständigkeit von Bedeutungen auf die Selbständigkeit/Unselbständigkeit von den entsprechenden Bedeutungsintentionen zurückgeführt. Wir fragen weiter, nach was für einem Kriterium man feststellt, ob eine gegebene Bedeutungsintention selbständig oder unselbständig ist? Der Test dafür ist, zu überprüfen, ob sie allein als ein vollständiger intentionaler Akt bestehen kann, ohne mit anderen Bedeutungsintentionen verküpft $\mathrm{zu}$ werden. Wenn ja, dann ist sie intentionalitätsunabhängig und somit selbständig, sonst ist sie intentionlaitätsabhängig und unselbständig.

Bei der Selbständigkeit/Unselbständigkeit der Bedeutungen handelt es sich also letztlich um die Intentionalitäts-Abhängigkeit/Unabhängigkeit der entsprechenden Bedeutungsintentionen. ${ }^{95}$ Nach (Charakterisierung*) „erbt“ eine selbständige Bedeutung die Intentionalität als ihr Eigentum von einer (sie instanziierenden)

\footnotetext{
${ }^{93}$ Die Bedeutung des Wortes ,und“ kann man so repräsentieren, dass sie zwei Leerstellen mit sich führt - als ein Vorschlag zum Symbolismus kann das Symbol „, ( )n und ( ) “" sein, wobei das Suffix n darauf hinweist, dass die beiden Leerstellen durch nominale Bedeutungen (wie „Sokrates“ und „Platon“) auszufüllen sind. Man kann die beiden Stellen unausgefüllt bleiben lassen, um die Bedeutung des herausgerissenen synkategorematischen Ausdrucks zu repräsentieren.

${ }^{94}$ Hua XIX/1, Logische Untersuchungen, S.320-321

${ }_{95}$ Hier sehen wir, wie Tugendhat bemerkt, dass Husserls gegenstandstheoretischer Ansatz über sprachliche Bedeutung „in eindrucksvoller Weise“ (Tugendhat 1976, S.167) sowohl für kategorematische als auch für synkategorematische Ausdrücke angewandt wird. Tugendhats Kritik ist, dass Husserls Bedeutungstheorie, die den Begriff von sprachlicher Bedeutung (besonders von singulären Termen, Prädikaten, Sätzen) ausschließlich (über den Intentionalitätsbegriff) durch den Gegenstandsbegriff betimmen will, unhaltbar ist, weil sie letztlich in zirkulärer Weise das Verstehen der Bedeutung des Satzes, was eben zu bestimmen ist, voraussetzt. Ausführlich über Tugendhats Kritik vgl. Tugendhat 1976, besonders die 9. und 10. Vorlesungen.
} 
intentionalitätsunabhängigen Bedeutungsintention, in diesem Sinne können wir sagen, dass selbständige Bedeutungen intentionalitätsunabhängig sind; im Gegensatz dazu kann eine unselbständige Bedeutung nur durch intentionlaitätsabhängige Bedeutungsintention, von der sie keine Intentionalität als Eigentum „erben“ kann, instanziiert werden, in diesem Sinne können wir sagen, dass unselbständige Bedeutungen intentionalitätsabhängig sind. Parallel zu der selbständigen bzw. intentionalitätsunabhängigen Bedeutungsintention haben wir auf der Bedeutungsebene die selbständige bzw. intentionalitätsunabhängige Bedeutung; parallel zu der unselbständigen bzw. intentionalitätsabhangigen Bedeutungsintention haben wir auf der Bedeutungsebene die unselbständige bzw. intentionalitätsabhangie Bedeutung. Die Unterscheidung von Selbständigkeit/Unselbständigkeit der Bedeutungen deckt sich mit der Unterscheidung von ihrer Intentionalitäts-Unabhängigkeit/-Abhängigkeit. Im folgenden Paragraphen verwerten wir die beiden äquivalenten Unterscheidungen, um Husserls Begriff von Bedeutungskategorie einzuführen.

\section{§10. Bedeutungskategorien und die Gesetze der Bedeutungskomplikation}

Das Forschungsergebnis der III. Logischen Untersuchung lehrt:

(1) „Unselbständige Inhalte[= Gegenstände - Anm.d.Verf. $]$ sind ... Inhalte[= Gegenstände], die nicht für sich, sondern nur als Teile von umfassenderen Ganzen Bestand haben können. Dieses Nicht-können hat seinen apriorischen Gesetzesgrund in

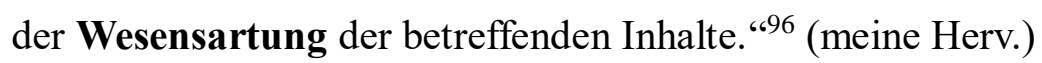

(2) „Zu jeder Unselbständigkeit gehört ein Gesetz, wonach überhaupt ein Inhalt der bezüglichen Art, sagen wir der Art $\alpha$, nur sein kann im Zusammenhang eines Ganzen $(\alpha \beta \ldots \mu)$, wo $\beta \ldots \mu$ Zeichen sind für bestimmte Inhaltsarten. Für bestimmte, betonten wir; denn kein Gesetz besagt bloß, daß zwischen der Art $\alpha$ und beliebigen anderen Arten Zusammenhang bestehe, daß also ein $\alpha$ nur überhaupt und gleichgültig welcher Ergänzung bedürfe, sondern zur Gesetzlichkeit gehört Bestimmtheit in der Artung des Zusammenhanges; abhängige und unabhängige Variable haben ihre durch feste Gattungs- oder Artcharakter umgrenzte Sphäre. “ 97 (meine Herv.)

\footnotetext{
${ }^{96}$ Ebd. S.319-320.

${ }^{97}$ Ebd.
} 
Nach Zitat (1) ist die Existenz eines unselbständigen Gegenstandes x abhängig von der Existenz anderer Gegenstände, die mit ihm zusammen ein umfassenderes Ganzes bilden. Diese Existenzabhängigkeit ist durch das Wesen X des fraglichen Gegenstandes $\mathrm{x}$ bestimmt, das heißt, dass alle Gegenstände $\mathrm{x}_{1}, \mathrm{x}_{2}, \mathrm{x}_{3}$ usw., die demselben Wesen X unterstehen, auch unselbständig sein müssen. Sie können also nur dann existieren, wenn sie mit anderen Gegenständen innerhalb eines umfassenderen Ganzen verbunden sind.

Nach Zitat (2) bestimmt das Wesen X eines unselbständigen Gegenstandes x nicht nur, dass x für seine Existenz mit anderen Gegenständen verbunden werden muss, sondern auch, dass x nur mit bestimmten Arten von Gegenständen verbunden werden kann. In diesem Sinne schreibt das Wesen X ein Gesetz für Verbindungsmöglichkeiten des Gegenstandes x vor: x kann nur mit Gegenständen verbunden werden, die bestimmten Wesen Y, Z usw. unterstehen. Z.B. schreibt das Wesen (Farbe) eines individuellen Rotmoments das Gesetz vor, dass dieses Rotmoment mit einem anderen Moment, das dem Wesen Ausdehnung untersteht, zu einer Einheit verbunden werden muss.

Diese allgemeine Lehre über die Existenz-Abhängigkeit von Gegenständen gilt mutatis mutandis für die Intentionalitäts-Abhängigkeit von Bedeutungen. Wir können Zitat (1) und (2) mit passender Änderung umformulieren und auf die Bedeutungssphäre anwenden:

(1') Unselbständige Bedeutungen sind Bedeutungen, die nicht für sich, sondern nur als Teile von umfassenderen Bedeutungen Intentionalität haben können. Dieses Nicht-können hat seinen apriorischen Gesetzesgrund in der Wesensartung der betreffenden Bedeutungen.

(2') $\mathrm{Zu}$ jeder Unselbständigkeit der Bedeutung gehört ein Gesetz, wonach überhaupt eine Bedeutung der bezüglichen Art, sagen wir der Art $\alpha$, nur Intentionalität haben kann im Zusammenhang eines Bedeutungsganzen $(\alpha \beta \ldots \mu)$, wo $\beta \ldots \mu$ Zeichen sind für bestimmte Bedeutungsarten.

Nach (1') kann eine unselbständige Bedeutung nur in Verbindung mit anderen Bedeutungen an einer Intentionalität teilnehmen. Nach (2') kann sie nicht mit beliebigen Bedeutungen, sondern nur mit Bedeutungen von bestimmten Arten verbunden werden. Z.B. die Bedeutung , ( ) $)_{\mathrm{n}}$ und ( $)_{\mathrm{n}}{ }^{\text {“98 }}$ kann sich nur mit zwei

\footnotetext{
${ }^{98}$ Das Suffix $\mathrm{n}$ weist darauf hin, dass dass die beiden Leerstellen durch nominale (substantivische) Bedeutungen (wie „Sokrates“ und „Platon“) auszufüllen sind
} 
substantivischen Bedeutungen $\mathrm{zu}$ einer neuen selbständigen Bedeutungseinheit zusammenschließen (wie „Sokrates und Platon“). Füllt man die Leerstellen mit ungeeigneten Bedeutungen aus, dann bekommt man nur einen „Bedeutungshaufen“ (wie ,aber und grün“) statt einer Bedeutungseinheit. ${ }^{99}$ Die „Wesensartung“ der Bedeutung (Bedeutungsart), die solche Komplikationsgesetze bestimmt, bezeichnet Husserl als Bedeutungskategorie. ${ }^{100}$ Es gibt verschiedene Bedeutungskategorien: konjunktionale Bedeutung („und“, „oder“), adjektivische Bedeutung (,,rot“, „rund“), präpositionale Bedeutung (,,in“) usw.

Selbständige Bedeutungen unterstehen auch bestimmten Bedeutungskategorien. Bedeutungskategorien der selbständigen Bedeutungen sind substantivische Bedeutungen, die in der Regel durch Namen (,,der blaue Himmel“) ausgedrückt werden, und propositionale Bedeutungen, die in der Regel durch Aussagesätze (,,der Himmel ist blau“) ausgedrückt werden. Eine selbständige Bedeutung (wie „Sokrates“), wie gesagt, besitzt schon Intentionalität, ohne mit anderen Bedeutung verbunden zu werden. Doch sie kann auch mit anderen Bedeutungen (,Sokrates“ mit ,und“ und „Platon“) zu einer umfassenderen selbständigen Bedeutungseinheit („Sokrates und Platon“) vereinigt werden, damit sie an einer umfassenderen Intentionalität teilnehmen kann.

Es gibt auch Fälle, wo mehrere Bedeutungen sich zu einer einheitlichen, aber unselbständigen Bedeutung zusammensetzen. Z.B. bilden die substantivische Bedeutung „Sokrates“ und die konjunktionale Bedeutung „( ) n und ( ) n“ $^{\text {“ die }}$

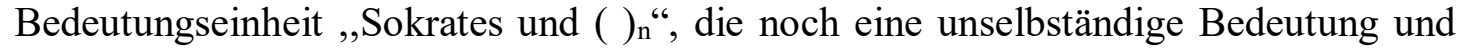
somit ergänzungsbedürftig ist.

Zusammenfassend gesagt: jede Bedeutung untersteht einer bestimmten Bedeutungskategorie, die eine substantivische oder propositionale oder adjektivische usw. sein kann. Man kann solche Bedeutungskategorien als wesentliche Gattungen der einzelnen Bedeutungen ansehen. In diesen Bedeutungskategorien wurzeln die Bedeutungsgesetze, die bestimmen, nach welchen Formen zwei oder mehr vorgegebene Bedeutungen von verschiedenen Bedeutungskategorien sich zu Einer Bedeutung vereinen, statt einen chaotischen Unsinn zu ergeben. Die Disziplin, die die Bedeutungskategorien und die damit zusammenhängenden Komplikationsgesetze der Bedeutungen untersucht, bezeichnet Husserl als reine Grammatik, apriorische

\footnotetext{
${ }^{99}$ Hua XIX/1, S.326: „Nur in gewissen, im voraus bestimmten Weisen passen die Bedeutungen zusammen und konstituieren wieder sinnvoll einheitliche Bedeutungen, während die übrigen kombinatorischen Möglichkeiten gesetzlich ausgeschlossen sind: sie ergeben nur einen Bedeutungshaufen statt einer Bedeutung." ${ }^{100}$ Ebd.
} 
Grammatik bzw. Formenlehre der Bedeutungen ${ }^{101}$.

\section{§11. Apriorische Grammatik vs. empirische Grammatik}

Husserl erachtet die durch Bedeutungskategorien bestimmten Komplikationsgesetze von Bedeutungen für apriorisch-grammatische Gesetze. ${ }^{102} \mathrm{Um}$ diese Gesetze genauer zu charakterisieren, können wir sie mit den empirischgrammatischen Regeln der Sprachen vergleichen.

Verschiedene Sprachen haben verschiedene grammatische Regeln. Wenn man z.B. die Bedeutung „Sokrates und Platon“ auf Deutsch ausdrücken will, schreibt man den Ausdruck ,,Sokrates und Platon“, während der Ausdruck „,Sokrates Platon und“ in der deutschen Sprache ungrammatisch ist. Die deutsche Grammatik hat die Regel, dass der Ausdruck, der die unselbständige Bedeutung ,und“ ausdrückt, zwischen den beiden Namen stehen muss. Im Lateinischen schreibt man „Sokrates Plato-que“, während der Ausdruck „Sokrates que Plato“ ungrammatisch ist. Die lateinische Grammatik hat also die Regel, dass der Ausdruck ,,-que“, der die Bedeutung „und“ ausdrückt, nach den Namen stehen muss. Ähnliche Unterschiede hinsichtlich der grammatischen Regeln von verschiedenen Sprachen sind überall zu zeigen. Solche grammatischen Regeln sind Konventionen der jeweiligen Sprache. Wir bezeichnen sie als konventionellgrammatische oder empirisch-grammatische Regeln.

Husserl zufolge gibt es auch grammatische Regeln, die nicht aus Konventionen stammen, sondern auf die Komplikationsgesetze von Bedeutungen zurückgehen. Z.B. erlaubt weder Latein noch Deutsch noch irgendeine andere Sprache einen Ausdruck, der die Bedeutung ,aber und Sokrates rot“"103 (durch ,aber und Sokrates rot“ auf

\footnotetext{
${ }^{101}$ Hua XIX/1, III. $L U, \S 13$.

${ }^{102}$ Hua XIX/1, S.345: ,Es gibt auch in der grammatischen Sphäre ein festes Maß, eine apriorische Norm, die nicht überschritten werden darf. Wie sich in der eigentlich logischen Sphäre das Apriorische als „reine Logik“ vom empirisch und praktisch Logischen sondert, ebenso sondert sich in der grammatischen Sphäre das sozusagen „rein“ Grammatische, d.h. eben das Apriorische (die „ideale Form“ der Sprache, wie man vortreffllich sagte) vom Empirischen. Beiderseits ist das Empirische teils durch die allgemeinen und doch nur faktischen Züge der Menschennatur bestimmt, teils auch durch die zufälligen Besonderungen der Rasse, näher des Volks und seiner Lebenserfahrung. Das Apriorische aber ist mindestens in seinen primitiven Gestaltungen hier und dort, wie überall sonst, ,,selbstverständlich“, ja geradezu trivial.“

${ }^{103}$ Streng genommen ist eine uneinheitliche Bedeutung, oder mit Husserl zu sagen, ein Bedeutungshaufen, gar keine Bedeutung. Manchmal kann man aber nicht auf den ersten Blick erkennen, ob ein gegebener Ausdruck grammatisch wohlgeformt ist, z.B. ein sehr langer und komplizierter Satz. In solchen Fällen erwartet man zuerst, dass der betreffende Satz eine einheitliche Bedeutung trägt. Aber nach grammatischen Analysen kann es sich herausstellen,
} 
Deutsch und ,sed -que Sokrates roseus“ auf Latein) ausdrückt, denn sie ist gar keine einheitliche Bedeutung, die ein sinnvoller Ausdruck irgendeiner Sprache tragen kann. Solche grammatischen Regeln gelten nicht nur für bestimmte, sondern für alle Sprachen; man kann sie als apriorisch-grammatische Regeln bezeichnen. ${ }^{104}$

Im Hinblick auf die Verschiedenheit zwischen empirisch-grammatischen Regeln und apriorisch-grammatischen Regeln schreibt Husserl:

Fragen wie nach den Gründen, warum in unserer Sprache gewisse Verknüpfungen gestattet sind und andere verwehrt, so werden wir allerdings $\mathrm{zu}$ einem sehr erheblichen Teil auf zufällige Sprachgewohnheiten und überhaupt auf Tatsächlichkeiten der bei einer Sprachgenossenschaft so, bei einer andern anders vollzogenen Sprachentwicklung hingewiesen.

Zum andern Teil stoßen wir aber auf den wesentlichen Unterschied der selbständigen und unselbständigen Bedeutungen, sowie auf die innig damit zusammenhängenden apriorischen Gesetze der Bedeutungsverknüpfung und Bedeutungsverwandlung, Gesetze, die sich in jeder entwickelten Sprache in der grammatischen Formenlehre und in einer zugehörigen Klasse von grammatischen Unverträglichkeiten mehr oder minder deutlich bekunden müssen. ${ }^{105}$

Die Komplikationsgesetze der Bedeutungen, die durch Bedeutungskategorien bestimmt werden und den apriorisch-grammatischen Regeln aller Sprachen zugrundeliegen, bilden das Forschungsgebiet der sogenannten Formenlehre der Bedeutungen bzw. der reinen Grammatik, in diesem Sinne ist die Formenlehre der Bedeutungen von Husserl auch als apriorische Grammatik bezeichnet. Im Vergleich

\footnotetext{
dass die Bedeutungen der Satzteile nicht zusammenpassen! Man erwartet eine einheitliche Bedeutung, aber bekommt nur einen Bedeutungshaufen. Man spricht von ,,uneinheitliche Bedeutung“ in solchen enttäuschenden Erwartungskontexten, außerhalb solcher Kontexte soll man die Rede von „uneinheitliche Bedeutung“ nicht ernst nehmen.

${ }^{104}$ Die Sinnlosigkeit von ,,aber und Sokrates rot“" scheint selbstverständlich zu sein und man könnte den Eindruck haben, dass die sogenannten apriorisch-grammatischen Regeln trivial und uninteressant sind. Allerdings ist die Trivialität für Husserl auch zu erklären: die Bedeutungskategorien von ,aber“, „und““, „Sokrates“, „,rot“" passen nicht zusammen. Husserl ist sogar der Meinung, dass ein systematisches Studium der Trivialität für Philosophie ,von allergrößtem Interesse und von nicht geringer Schwierigkeit [ist].“ (IV. LU, S.345) Außerdem gibt es auch kompliziertere „Bedeutungshaufen“, deren Sinnlosigkeit nicht auf den ersten Blick zu erkennen, sondern nur durch bedeutungskategorische Analysen zu zeigen ist. In solchen Fällen ist die Sinnlosigkeit nicht mehr trivial.

${ }^{105}$ Ebd., S.335-336.
} 
dazu untersuchen die Grammatiken in normaler Rede nur die konventionellgrammatischen Regeln der jeweiligen Sprachen, wir können diese Grammatiken als empirische Grammatiken bezeichnen. ${ }^{106}$

[Man spricht auch in der chomkyschen generativen Grammatik (anders genannt: transformational-generative Grammatik) von Universalien der Menschensprachen. Ein systematischer Vergleich zwischen der chomkyschen Grammatik und Husserls apriorischer Grammatik würde den Rahmen der vorliegenden Arbeit sprengen, hier möchte ich die Parallele und Unterschied zwischen den beiden kurz erwähnen. Die chomkyschen Syntaktiker entwickeln eine Reihe von Regeln (Phrase Structure Rules genannt), um die syntaktische Struktur der wohlgeformten Sätze einer bestimmten Sprache zu repräsentieren, und entwerfen eine Reihe Parameter-Regeln, um die universalen Charaktere von allen Menschensprachen zu modellieren. Diese Regeln verwenden, ähnlich wie bei Husserl, syntaktische Kategorien wie NP (noun phrase), AdjP, AdvP usw. Letztlich ist also auch das Wohlgeformtsein der Sätze von Menschensprachen universal durch syntaktische Formen (bei Husserl die Bedeutungskategorien, bei der generativen Grammatik die syntaktische Kategorien) beschränkt. In Gegensatz zu Husserl werden die Regeln bei chomskyschen Syntaktikern nicht einfach als apriorisch und somit universal für Menschen interpretiert, sondern sie werden als biologisch-genetische Regeln, die in dem postulierten menschlichen Sprachorgan (auch Universal Grammar genannt) eingeboren sind, angesehen und sind somit aus biologischen Gründen für Menschen universal. Universale Charaktere in syntaktischer Hinsicht aller Menschensprachen sind also bei Husserl und chomskyschen Syntaktikern anerkannt, aber verschieden interpretiert. $\left.{ }^{107}\right]$

\section{§12. Modifikationsgesetze von Bedeutungen I}

Oben diskutieren wir hauptsächlich die Verknüpfungsgesetze der Bedeutungen. Nach diesen Gesetzen sind Ausdrücke wie ,oder und aber“ ungrammatisch aus

\footnotetext{
${ }^{106}$ Vgl. ebd., S.344-348.

${ }^{107}$ Eine systemaische und gut verständliche Einführung in generative Grammatik ist Andrew Carnie: Syntax. A Generative Introduction, Wiley-Blackwell, West Sussex 2013. Zu einem ausführlicheren Vergleich zwischen Husserls apriorischer Grammatik und generativer Grammatik sieh James M. Edie 1977.
} 
apriorischen Gründen. Denn die konjunktionale Bedeutung ,( ) und ( )n ${ }^{\text {“ }}$ kann nur mit zwei substantivischen (nominalen) Bedeutungen verknüpft werden. Doch man könnte einwenden, dass Ausdrücke wie „oder und aber“ in manchen Kontexten auch eine einheitliche Bedeutungen tragen - ein solcher Fall passiert in Sätzen wie ,oder und aber sind konjunktionale Bedeutungen“. Ähnliche Fälle liegen auch überall vor, wo ein normalerweise nicht-nominaler Ausdruck an einer Stelle, die normalerweise durch einen nominalen Ausdruck auszufüllen ist, auftritt.

Diese Fälle scheinen den apriorisch-allgemeinen Status der Komplikationsgesetze, nach denen nur Bedeutungen von bestimmten Bedeutungskategorien verknüpft werden können, zu erschüttern.

Husserl erwidert, dass die Ausdrücke „oder“ und „aber“ in den fraglichen Kontexten nicht mehr ihre normalen Bedeutungen haben. Die Scholastiker sprechen von der Operation der suppositio materialis, wodurch ein Ausdruck nicht mehr seinen normalen Gegenstand, sondern ihn selbst bezeichnet. Z.B. nennt der Ausdruck „Sokrates“ im Satz ,Sokrates ist ein Eigenname“ nicht die Person Sokrates, sondern den Eigennamen selbst. ${ }^{108}$ Ein Analogon der suppositio materialis liegt vor, wenn ein Ausdruck nicht mehr seinen normalen Gegenstand, sondern seine normale Bedeutung bezeichnet wie im Satz ,Sokrates ist eine Eigenbedeutung“. Dabei wird natürlich auch die normale Bedeutung des Ausdrucks durch eine neue Bedeutung ersetzt.

Die Bedeutsamkeit von Sätzen wie ,oder und aber sind konjunktionale Bedeutungen“ besteht eben darin, dass hier die Ausdrücke „oder“ und ,aber“ dem Analogon der suppositio materialis unterliegen. Manchmal hat die neue Bedeutung des Ausdrucks dieselbe Bedeutungskategorie wie die normale - die neue Bedeutung des Ausdrucks „Sokrates“ untersteht der Bedeutungskategorie substantivische Bedeutung wie seine normale Bedeutung; manchmal wird sogar die Bedeutungskategorie verändert - in unserem Beispiel ist die neue Bedeutung des Ausdrucks ,oder“ keine konjunktionale Bedeutung (wie die normale), sondern eine substantivische

\footnotetext{
${ }^{108}$ Hua XIX/1: Logische Untersuchungen, S.331.
} 
Bedeutung. ${ }^{109}$

Wir können das Modifikationsgesetz der suppositio materialis wie folgt formulieren:

(Das Modifikationsgesetz der suppositio materialis)

Die Bedeutung jedes Ausdrucks lässt sich in eine substantivische Bedeutung, die sich auf die erstere bezieht, verwandeln.

Danach sind die Fälle wie ,oder und aber sind konjunktionale Bedeutungen" keine Gegenbeispiele gegen den apriorisch-allgemeinen Status der durch Bedeutungskategorien bestimmten Komplikationsgesetze; sie sind nur durch das Gesetz der suppositio materialis vermittelte Einzelfälle der Komplikationsgesetze. Aus diesem Grund kann man das Gesetz der suppositio materialis für ein ergänzendes Gesetz halten, das die Formenlehre der Bedeutungen neben den Verknüpfungsgesetzen auch in Betracht ziehen muss.

\section{§13. Modifikationsgesetze von Bedeutungen II}

Neben dem Gesetz der suppositio materialis gibt es noch andere Modifikationsgesetze, von denen Husserl in der $I V$. $L U$ gesprochen hat. Um diese anderen Modifikationsgesetze darzustellen, muss man zuerst die Begriffe Kernstoff, Kernform und syntaktische Form erläutern. ${ }^{110}$

Diese Begriffe lassen sich durch Beispielsanalysen illustrieren. Betrachten wir die folgenden Sätze:

(a) Die Kugel ist rot;

(b) Die Kugel ist grün;

(c) Die rote Kugel ist klein;

(d) Dieses Rot ist eine Farbe.

Vergleichen wir zuerst (a) und (b), in beiden Sätzen haben die Bedeutungen „,rot“ und „grün“ eine gemeinsame syntaktische Form, denn beide

\footnotetext{
109 Ebd.

${ }^{110}$ Ebd., S.332-334. Dazu vgl. auch Hua XVII: Formale und transzendentale Logik. Versuch einer Kritik der logischen Vernunft, hrsg. von Paul Janssen, Martinus Nijhoff, Den Haag 1974, 299-312.
} 
übernehmen die prädikative Funktion in den jeweiligen Propositionen. Angesichts des syntaktischen Stoffs ${ }^{111}$ unterscheiden sie sich voneinander - obwohl sie dieselbe Kernform, nämlich die adjektivische (Kern-)Form haben, aber ihre Kernstoffe ${ }^{112}$ haben verschiedene sachliche Inhalte und sind somit verschieden: Das „rot“ in (a) hat etwas mit roter Farbe zu tun, während das ,grün“ in (b) von grüner Farbe handelt.

Vergleichen wir (a) und (c). Die Bedeutung ,,rot" funktioniert in ihnen jeweils prädikativ und attributiv und somit ist von verschiedenen syntaktischen Formen umkleidet. Aber sie hat in beiden Propositionen denselben syntaktischen Stoff - in beiden Fällen ist die Kernform adjektivisch und bezieht sich der Kernstoff auf dasselbe Sachliche (rot).

Die beiden Bedeutungen ,rot“" in (a) und (d) haben verschiedene syntaktische Formen, nämlich prädikative Form in (a) und Subjektsform in (d). Die Kernformen gehen auch auseinander: in (a) ist „rot“ adjektivisch, in (d) substantivisch. Nur der Kernstoff ist gleich: beide handeln von roter Farbe. ${ }^{113}$

Besonders zu bemerken ist der Unterschied zwischen Kernform und syntaktischer Form der Bedeutungen. Syntaktische Formen sind bestimmt durch die Funktionen (Subjektsfunktion, Objektsfunktion, prädikative Funktion, attributive Funktion usw.), die eine Bedeutung innerhalb einer größeren Bedeutungseinheit übernimmt, deshalb sind sie auch als Funktionsformen ${ }^{114}$ bezeichnet. Kernformen sind die

\footnotetext{
${ }^{111}$ Zwei Bedeutungen haben den gleichen syntaktischen Stoff genau dann, wenn sowohl ihre Kernformen als auch ihre Kernstoffe gleich sind. Dazu mehr vgl. IV. $L U, \S 11$.

${ }^{112}$ Grob gesagt ist der Kernstoff dasjenige Element der Bedeutung, das ihre Kernform ausfüllt, um den sachlichen Inhalt der Bedeutung zu bestimmen. Wenn wir z.B. bloß die adjektivische Kernform einer Bedeutung durch das Symbol [ ] ]adj repräsentieren, dann wird diese Bedeutung nur unvollständig spezifiziert, denn ihr sachlicher Inhalt bleibt noch unbestimmt. Dazu können wir die Angabe des Kernstoffs ergänzen, indem wir in die Leerstelle „grün“, ,rot“ usw. einsetzen und somit das bloß formale Symbol [ ] ]adj in inhaltsreiche Symbole wie [rot]adj, [grün] adj usw. materialisieren. Die Bedeutungen [rot] $]_{\text {adj }}$ und [grün] adj unterscheiden sich in Hinsicht auf den Kernstoff, weil sie verschiedene sachliche Inhalte haben: [rot] $]_{\mathrm{adj}}$ hat etwas mit der roten Farbe zu tun, während [grün] adj von der grünen Farbe handelt.

${ }^{113}$ Der Begriff von Kernstoff lässt sich vielleicht durch den Wortfeldbegriff, der oft im Sprachkurs als Mnemotechnik des Vokabulars eingeführt wird, illustrieren. Ein Wortfeld ist eine Gruppe von Wörtern oder Phrasen, die sich in ihrer Bedeutung sehr ,ähnlich“ sind. Z.B. gehören die Wörter/Phrasen „eilig“, „Eile“, „,es eilig haben“ zu einem Wortfeld, in ähnlicher Weise gehören „Röte“, „rot“ zu einem Wortfeld. Das Prinzip, das die Wörter/Phrasen zu ein und demselben Wortfeld vereinigt, scheint aber vag und intuitiv zu sein: man kann nur vag sagen, „eilig“, „Eile“, ,es eilig haben“ haben etwas mit Eile zu tun; ,Röte“ und „,rot“ haben etwas mit roter Farbe zu tun. Zu den Begriffen von Kernstoff, Kernform und syntaktischer Form vgl. auch Vittorio De Palma, „Die Syntax der Erfahrung. Zu den sachhaltigen Voraussetzungen des Logischen und des Sprachlichen“, in: Meaning and Language: Phenomenological Perspectives, hrsg. von Filip Mattens, Springer 2008, S.130-132.

${ }^{114}$ Edmund Husserl, Erfahrung und Urteil. Untersuchungen zur Genealogie der Logik, redigiert und herausgegeben von Ludwig Landgrebe, Classen \& Goverts GmbH, Hamburg 1948, S.248.
} 
Bedeutungskategorien der betreffenden Bedeutungen. Eine Bedeutung kann in verschiedenen Zusammenhängen verschiedene Funktionen und somit verschiedene syntaktische Formen übernehmen, ohne ihre Bedeutungskategorie oder Kernform zu verändern. Z.B. funktioniert die adjektivische Bedeutung ,,rot“ in (a) prädikativ, in (c) attributiv. Auf der Ausdrucksebene existiert ein entsprechendes Phänomen: ein Adjektiv kann sowohl als Prädikat als auch als Attribut in einem Satz fungieren.

Manche Bedeutungen lassen sich so modifizieren, dass die neue Bedeutung, die durch die Modifikation entsteht, eine andere Kernform oder Bedeutungskategorie als die der originalen Bedeutung trägt, während der Kernstoff unverändert bleibt. Die Gruppe solcher Modifikationen bezeichne ich als K-Modifikationen. Dafür liefern die ,rot“-Bedeutungen in (a) und (d) ein Beispiel: In beiden Sätzen haben die „rot“Bedeutungen denselben sachlichen Inhalt (beide handeln von der roten Farbe), aber sie tragen verschiedene Kernformen; man kann sagen, die ,rot“-Bedeutung in (a) nominalisiert sich in die ,rot"-Bedeutung in (d), oder umgekehrt, die ,rot“-Bedeutung in (d) adjektivisiert sich in die ,,rot"s-Bedeutung in (a). ${ }^{115}$

K-Modifikationen sind auch durch Gesetze geregelt. Ein K-Modifikationsgesetz, von dem Husserl oft Gebrauch macht, ist das Gesetz der K-Nominalisierung von Propositionen:

(Das Gesetz der K-Nominalisierung von Propositionen)

Jede propositionale Bedeutung lässt sich durch K-Modifikation in eine substantivische Bedeutung verwandeln.

Durch die K-Nominalisierung kann man die Proposition ,,die Kugel ist rot“ in substantivische Bedeutung „die Tatsache, dass die Kugel rot ist“ verwandeln, damit die originale Proposition innerhalb einer anderen Proposition wie ,[die Tatsache, dass die Kugel rot ist $]_{\mathrm{n}}$, gefällt mir“" als Bestandteil fungiert.

Auch nach dem Modifikationsgesetz der suppositio materialis kann man jede propositionale Bedeutung in eine substantivische Bedeutung verwandeln. Doch diese Verwandlung ist keine K-Nominalisierung der Proposition. Denn die Modifikation der

\footnotetext{
${ }^{115}$ Hua XIX/1: Logische Untersuchungen, S.333-334.
} 
suppositio materialis erfordert, dass sich die neue Bedeutung auf die originale Bedeutung bezieht - dabei ist der Kernstoff schon verändert, was eine K-Modifikation nicht erlaubt! Zum Beispiel wird die Proposition ,die Kugel ist rot“ durch Modifikation der suppositio materialis in die substantivische Bedeutung „die Proposition ,die Kugel ist rot" “, die sich auf die originale Proposition bezieht, verwandelt; im Vergleich dazu resultiert aus der K-Nominalisierung die substantivische Bedeutung ,die Tatsache, dass die Kugel rot ist", die sich nicht auf die originale Proposition, sondern auf deren Gegenstand (die Tatsache) bezieht; die resultierenden Bedeutungen der beiden Modifikationen sind ganz verschieden! Die Gefahr, beide Modifikationen zu verwechseln, besteht, weil die Erzeugnisse beiderseits substantivische Bedeutungen sind. Man muss die Gefahr berücksichtigen und die Verwechselung vermeiden. ${ }^{116}$

Die Modifikationsgesetze, als Ergänzung zu Verknüpfungsgesetzen, sind auch apriorische Komplikationsgesetze von Bedeutungen. In diesem Sinne spricht Husserl von zwei Gesetzesgruppen, nämlich den ,apriorischen Gesetzen der Bedeutungsverknüpfung und Bedeutungsverwandlung“117. Die reine Grammatik untersucht beide Gruppen der apriorisch-grammatischen Gesetze.

\section{§14. Zusammenfassung des Kapitels und das Grammatikalitätsphänomen der}

\section{Bedeutungen}

Durch obige Ausführungen haben wir einen Überblick über Husserls Konzeption der „reinen Grammatik“ gewonnen. Wir nahmen den Ausgang von einer grammatischen Unterscheidung auf der Ausdrucksebene, nämlich der Unterscheidung zwischen kategorematischen und synkategorematischen Ausdrücken. Husserls Versuch, die bedeutungsmäßigen Eigenheiten von beiden Ausdrücksarten zu charakterisieren, führt zu der Unterscheidung zwischen selbständigen und unselbständigen Bedeutungen.

Sowohl selbständige als auch unselbständige Bedeutungen sind bestimmten Bedeutungskategorien untergeordnet. In den Bedeutungskategorien wurzeln die

\footnotetext{
${ }^{116}$ Nach dem Zusatz im [§13.] von $I V . L U$ gilt die bloße Veränderung der syntaktischen Form von Bedeutungen (wie „rot“ in (a) und (c)) auch als eine Art Bedeutungsmodifikation. Man muss also noch den Unterschied zwischen K-Modifikation und Modifikation der syntaktischen Form berücksichtigen.

${ }^{117}$ Hua XIX/1: Logische Untersuchungen, S.336.
} 
Komplikationsgesetze, die entscheiden, ob und wie zwei oder mehr vorgegebene Bedeutungen von bestimmten Bedeutungskategorien sich zu Einer Bedeutung vereinen, statt einen chaotischen Unsinn zu ergeben.

Husserl unterscheidet zwei Typen grammatischer Regeln irgendeiner bestimmten Sprache: Einerseits die Regeln, deren Gründe in der Sprachgewohnheiten oder Konventionen liegen; andererseits die Regeln, die nicht aus bloßen Konventionen stammen, sondern einen apriorischen Grund in den durch Bedeutungskategorien bestimmten Komplikationsgesetzen haben. Diese Gesetze gelten für alle Sprachen; oder umgekehrt, jede Sprache verwirklichen diese Gesetze. Die Art und Weise, in der diese Gesetze sprachlich verwirklicht werden, sind bei verschiedenen Sprachen verschieden. Deshalb spricht Husserl von einem ,,idealen Gerüst“", das die tatsächlichen Sprachen in verschiedenen Weisen ausfüllen. ${ }^{118}$

Aus diesen Gründen nennen wir solche Komplikationsgesetze apriorischgrammatische Gesetze. Im Vergleich zu den empirisch-grammatischen Regeln sind sie bei zwei Aspekten ausgezeichnet: (1) Im prägnanten Sinne sind sie nicht Komplikationsgesetze von Ausdrücken irgendeiner Sprache, sondern Komplikationsgesetze von Bedeutungen; doch sie begründen eine Gruppe grammatischer Regeln der Sprachen, deswegen sagen wir, dass sie auch für Sprachen gelten; (2) sie sind nicht nur grammatische Gesetze für eine bestimmte Sprache wie die empirisch-grammatischen Regeln, sondern sie gelten für alle Sprachen. Husserl konzipiert eine „Formenlehre der Bedeutungen“ oder „,reine Grammatik“, deren Aufgabe ist, diese apriorisch-grammatischen Gesetze zu untersuchen.

Die Komplikationsgesetze der Bedeutungen verteilen sich in die Gesetze der Bedeutungsverknüpfung und die der Bedeutungsverwandlung. Beide Gesetzesgruppen gehören zum Forschungsgebiet der reinen Grammatik.

Zum Schluss des Kapitels möchte ich die Grammatikalitätsthese der Bedeutungen und somit die Rede vom Grammatikalitätsphänomen (bezüglich) der Bedeutungen einführen:

${ }^{118}$ Ebd., S.347-348. 
(Die Grammatikalitätsthese der Bedeutungen)

Ausdrucksbedeutungen sind gewissen durch Bedeutungskategorien bestimmten grammatischen Gesetzen unterworfen. Das bezeichnen wir als das Grammatikalitätsphänomen (bezüglich) der Bedeutungen. 


\section{KAPITEL}

\section{REINE GRAMMATIK UND FORMALE LOGIK}

\section{§15. Reine Grammatik im Rahmen der formalen Logik}

Im letzten Kapitel haben wir die reine Grammatik mit empirischen Grammatiken verglichen, wobei die reine Grammatik sich durch zwei Eigentümlichkeit auszeichnet: (1) ihre Forschungsgebiet bilden die Bedeutungen; (2) die Gesetze, die sie untersucht, sind apriorische Gesetze. In diesem Sinne ist die reine Grammatik eine apriorische Disziplin der Bedeutungen. Doch für Husserl ist reine Grammatik nur eine Teildisziplin der formalen Logik von Bedeutungen. Denn Husserl zufolge ist die formale Logik von Bedeutungen, oder einfach die formale Apophantik, eine Wissenschaft, die die Bedeutungsformen und die dazugehörigen Gesetze untersucht. Doch die Bedeutungsformen betimmen nicht nur die Komplikationsgesetze von Bedeutungen, sondern auch ihre Geltungsgesetze wie Gesetze der Schlussfolgerung. In diesem Sinne enthält die formale Apophantik auch andere apriorische Disziplinen von Bedeutungen. Außerdem bildet die formale Apophantik nur einen Teil der formalen Logik überhaupt, die noch die sogenannte formale Ontologie enthält. In diesem Kapitel versuche ich zu erläutern, wie Husserl den Unterschied und Zusammenhang zwischen der reinen Grammatik und anderen formal-logischen Disziplinen konzipiert.

\section{§16. Reine Formenlehre und reine Geltungslehre der Bedeutungen}

Die späteren Analysen vorwegnehmend können wir sagen, dass die reine Grammatik die unterste Stufe $^{119}$ der formalen Logik ausmacht. In der IV. LU beschreibt Husserl sie als Fundament der formalen Logik:

In der reinen Logik der Bedeutungen, deren höheres Ziel in Gesetzen gegenständlicher Geltung der Bedeutungen liegt, soweit solche Geltung durch die reine Bedeutungsform bedingt ist, bildet die Lehre vom Wesensbau der

\footnotetext{
${ }^{119}$ Vgl. [§20] der vorliegenden Arbeit.
} 
Bedeutungen und den Gesetzen ihrer Formenbildungen das notwendige Fundament. ${ }^{120}$

In diesem Zitat erwähnt Husserl zwei Disziplinen im Rahmen der Logik von Bedeutungen: (1) die Disziplin, die ,den Wesensbau der Bedeutungen und die Gesetze ihrer Formenbildungen“ untersucht und (2) die Disziplin, die die „Gesetze gegenständlicher Geltung der Bedeutungen“" untersucht. Die erstere ist eben reine Grammatik - sie hat die Aufgabe, aufgrund der apriorisch-grammatischen Gesetze sinnvolle Ausdrücke von sinnlosen Ausdrücken zu unterscheiden, oder mit Husserls eigenen Worten, Sinn von Unsinn zu unterscheiden; in den Logischen Untersuchungen ist sie auch als Formenlehre der Bedeutungen bezeichnet. Die letztere bezeichnet Husserl als Geltungslehre der Bedeutungen; ihre Aufgabe ist Konsistenz von Widersinn zu unterscheiden. ${ }^{121}$

Wir sprechen von Sinn/Unsinn und Konsistenz/Widersinn. Sinn/Unsinn bestimmt das Thema der Formenlehre, und Konsistenz/Widersinn bestimmt das Thema der Geltungslehre. Eine Interpretation der beiden Begriffspaare hilft uns, Husserls Auffassung über den Unterschied und Zusammenhang zwischen den beiden Disziplinen genauer nachzuvollziehen. Im Folgenden möchte ich erklären, was unter diesen beiden Begriffspaaren zu verstehen ist und wie sie die jeweiligen Themen der beiden Disziplinen von Bedeutungen stichwortig darstellen.

\section{§17. Sinn und Unsinn}

In der $I$. $L U$ hat Husserl mehrere Arten von sinnlosen Ausdrücken betrachtet. Husserl bemerkt, dass man in vieldeutiger Weise von der Sinnlosigkeit der folgenden Audrücke spricht: (1) Abracadabra; (2) goldener Berg; (3) rundes Viereck.

Husserl zufolge muss man diese äquivoke Rede der Sinnlosigkeit vermeiden. ${ }^{122}$ Denn die Gründe, aus denen die fraglichen Ausdrücke für sinnlos erachtet werden, sind verschieden. Das heißt, sie sind nicht in demselben Sinne ,,sinnlos“. Ausdruck (1) ist

\footnotetext{
${ }^{120}$ Hua XIX/1: Logische Untersuchungen, S.337.

${ }^{121}$ Ebd., S.334-335, 342-344.

122 Ebd., S.59: „Doch wichtiger ist es für uns, die in ihren Folgen sehr schädlichen Äquivokationen der Rede von Bedeutung und Sinn, bzw. der Rede von bedeutungslosen oder sinnlosen Ausdrücken genau auseinander zu legen.“
} 
sinnlos, weil er eigentlich gar kein Ausdruck ist, sondern nur eine Reihe von Buchstaben ${ }^{123}$; Ausdrücke (2) und (3) sind sinnlos in dem Sinne, dass sie gegenstandslos sind ${ }^{124}$ - (2) ist aus empirischen Gründen gegenstandslos, während (3) aus apriorischen Gründen kein Gegenstand entsprechen kann.

Doch (2) und (3) sind unter einem formalen Aspekt sinnvoll, denn die Form von ihnen ist eine von der reinen Grammatik anerkannte gesetzliche Formbildung. Nach der reinen Grammatik kann sich irgendeine substantivische Bedeutung $\mathrm{X}_{\mathrm{n}}{ }^{125}$ mit irgendeiner adjektivischen Bedeutung $\mathrm{Y}_{\mathrm{adj}}{ }^{126} \mathrm{zu}$ einer umfassenderen substantivischen Bedeutung $\left[\mathrm{Y}_{\mathrm{adj}} \mathrm{X}_{\mathrm{n}}\right]_{\mathrm{n}}{ }^{127}$ zusammsetzen, egal, ob der „Inhalt“ der erzeugten Bedeutung gegenstandslos und sogar absurd ist. Die reine Grammatik kümmert sich nur um die formale Bedingung des Sinnvollseins - solange die Bedeutung eine zulässige Form hat, dann ist sie eine formal einheitliche Bedeutung. Ausdrücke (2) und (3) haben die zulässige Form $\left[Y_{\text {adj }} X_{n}\right]_{n}$ und erfüllt diese Bedingung, deshalb sind sie vom Standpunkt der reinen Grammatik aus sinnvolle Ausdrücke; mit Husserls eigenen Worten, sie sind (formal-)sinnvolle Ausdrücke (vs. unsinnige Ausdrücke) oder einfach (formaler) Sinn (vs. Unsinn).

Ziehen wir noch einen Ausdruck in Betracht:

(4) goldenes oder.

Im Gegensatz zu (2) und (3) ist seine Bedeutungsform $\left[\mathrm{Y}_{\mathrm{adj}} \mathrm{X}_{\mathrm{kon}}\right]_{?}^{128}$ unter der reingrammatischen Perspektive ungesetzlich. Denn nach der reinen Grammatik gibt es keine einheitliche Bedeutung, die durch eine adjektivische und eine konjunktionale Bedeutung zusammengesetzt wird. (1) und (4) sind beide sinnlos, mit der Kleinigkeit,

\footnotetext{
${ }^{123}$ Ebd.: „Ein bedeutungsloser Ausdruck ist also, eigentlich zu reden, überhaupt kein Ausdruck; bestenfalls ist er ein Irgendetwas, das den Anspruch oder Anschein erweckt, ein Ausdruck zu sein, während es dies, näher besehen, gar nicht ist."

${ }^{124}$ Husserl bemerkt, dass man im allgemeinen Ausdrücke wie (2) nicht als sinnlos, sondern als gegenstandlos bezeichnet. Dagegen liebt man es, Ausdrücke wie (3) als sinnlos (im Sinne von absurd) zu bezeichnen. Darüber vgl. Hua XIX/1, S.60: ,,Wird die Bedeutung, wie soeben, mit der Gegenständlichkeit des Ausdrucks identifiziert, so ist ein Name wie goldener Berg bedeutungslos. Allgemein unterscheidet man hier aber die Gegenstandslosigkeit von der Bedeutungslosigkeit. Dagegen liebt man es, widerspruchsvolle ... Ausdrücke, wie rundes Viereck, als sinnlose zu bezeichnen ..."

${ }^{125}$ Das Suffix $n$ weist darauf hin, dass die Bedeutung eine nominale (substantivische) Bedeutung ist.

${ }^{126}$ Das Suffix adj weist darauf hin, dass die Bedeutung eine adjektivische Bedeutung ist.

${ }^{127}$ Das Suffix $n$ nach der eckigen Klammer weist darauf hin, dass die Bedeutung, die durch die Bedeutungen in der eckigen Klammer zusammengesetzt wird, eine nominale (substantivische) Bedeutung ist.

${ }^{128}$ Das Suffix ? weist darauf hin, dass die Zusammensetzung der in der eckigen Klammer stehenden Bedeutungen keine einheitliche Bedeutung ergibt.
} 
dass (4) mindestens ein Haufen von bedeutsamen Ausdrücken ist und prätendiert, eine einheitliche zusammengesetzte Bedeutung zu haben. In Husserls Terminologie ist Ausdruck (4) (formal-)unsinnig oder (formaler) Unsinn, während (1) einfach durch und durch sinnlos ist.

Betrachten wir wiederum ein Beispiel:

(5) blaue Bedeutung.

Dieser Ausdruck ist in üblicher Rede auch für sinnlos gehalten, denn er macht einen Kategorienfehler - Blausein ist eine Eigenschaft, die nur physische Gegenstände haben können, und bei idealen Gegenständen kann vom Blausein gar keine Rede sein. Laut Husserls Ontologie gehören Bedeutungen zu einer besonderen Seinsregion, wo man nicht vernünftig fragen darf, ob ein dazugehöriger Gegenstand blau ist oder nicht; diese Frage kann man nur in Seinsregion der physichen Gegenstände stellen. Im Vergleich dazu implizieren die Frage, ob ein Berg golden ist, und die Frage, ob ein Viereck rund ist, und somit die Ausdrücke (2) und (3), keinen Kategorienfehler. Denn ein Berg gehört zur Seinsregion der physichen Gegenstände, und physische Gegenstände können blau sein; ein Viereck gehört zur Seinsregion der geometrischen Gegenstände, und geometrische Gegenstände können rund sein. Für Husserl sind kategorial-fehlerhafte Ausdrücke wie (5) material-unsinnig oder einfach materialer Unsinn.

Doch für die reine Grammatik haben (2), (3) und (5) alle einheitliche Bedeutung und sind deshalb (formal-)sinnvolle Ausdrücke. ${ }^{129}$ Denn sie alle ,,instanziieren“ die gesetzmäßige Bedeutungsform $\left[\mathrm{Y}_{\mathrm{adj}} \mathrm{X}_{\mathrm{n}}\right]_{\mathrm{n}}$. Die reine Grammatik beschäftigt sich nur mit der formalen Sinnhaftigkeit/Sinnlosigkeit der Bedeutungen und kümmert sich nicht darum, ob sie inhaltlich sinnlos (gegenstandslos oder material-unsinnig) sind. Zu entscheiden, ob eine gegebene Bedeutung inhaltlich sinnvoll ist oder nicht, ist keine Aufgabe, die die reine Grammatik übernehmen soll und übernehmen kann. Dafür muss man über die reine Grammatik hinausgehen und sich an Wissenschaften wie

\footnotetext{
${ }^{129}$ Hua XIX/1, S.327: „Bei den freien Vertauschungen von Materien innerhalb ihrer Kategorie mögen falsche, dumme, lächerliche Bedeutungen (ganze Sätze oder mögliche Satzglieder) resultieren, aber notwendig resultieren einheitliche Bedeutungen, bzw. grammatische Ausdrücke, deren Sinn sich einheitlich vollziehen läßt. Sowie wir die Kategorien überschreiten, ist das nicht mehr der Fall.“
} 
Geographie (für Ausdruck (2)), Geometrie (für Ausdruck (3)), Ontologie (für Ausdruck (5)) usw. wenden.

Mit dem Begriffspaar Sinn/Unsinn meint Husserl meistens die formale Sinnhaftigkeit/Sinnlosigkeit von Ausdrücken. Ob ein Ausdruck formal-sinnvoll oder formal-sinnlos ist, hängt nur davon ab, ob seine Bedeutung eine Form hat, die die apriorisch-grammatischen Gesetze erlauben. Die reine Grammatik untersucht eben diese apriorisch-grammatischen Gesetze und übernimmt somit die Aufgabe, Sinn von Unsinn zu unterscheiden.

\section{§18. Konsistenz und Widersinn}

Wie oben schon erwähnt, ist die reine Grammatik nicht die einzige Disziplin von Bedeutungen. In der $I V . L U$ vergleicht Husserl die reine Grammatik mit der Geltungslehre der Bedeutungen. Beide Disziplinen haben das gemeinsame Forschungsgebiet von Bedeutungen, aber sie haben verschiedene Aufgaben. Die reine Grammatik, wie bereits dargelegt, legt den Schwerpunkt auf die Unterscheidung zwischen Sinn und Unsinn; die Geltungslehre der Bedeutungen kümmert sich um eine andere Unterscheidung, nämlich die zwischen Konsistenz und Widersinn. In diesem Paragraphen erklären wir das Begriffspaar Konsistenz/Widersinn.

Betrachten wir die folgenden Ausdrücke:

(1) rundes Viereck;

(2) rundes und nicht-rundes Viereck.

Beide Ausdrücke sind widersprüchlich, mit Husserls eigenen Worten, sie sind widersinnig oder einfach Widersinn. Doch ihre Weisen von Widersinnigkeit sind verschieden. Wir können die Bedeutungsformen von (1) und (2) wie folgt repräsentieren:

(1') $\left[Y_{\text {adj }} X_{n}\right]_{n}$ (rundes Viereck)

(2’) $\left[\left[Y_{\text {adj }} \text { und }\left[\text { nicht- } Y_{\text {adj }}\right]_{\text {adj }}\right]_{\text {adj }} X_{n}\right]_{n}$ (rundes und nicht-rundes Viereck)

Allein an der Bedeutungsform vom Ausdruck (1) können wir nicht erkennen, dass er widersinnig ist. Viele Bedeutungen wie „,blauer Himmel“, „rotes Auto“ haben dieselbe 
Form $\left[\mathrm{Y}_{\mathrm{adj}} \mathrm{X}_{\mathrm{n}}\right]_{\mathrm{n}}$, und sie sind konsistent. Um $\mathrm{zu}$ entscheiden, ob Ausdruck (1) widersinnig ist, muss man über die Bedeutungsform hinausgehen - man muss den Gegenstand der Bedeutung (hier das Viereck) betrachten und Geometrie betreiben. In derselben Weise sind Ausdrücke wie „,reguläres Dekaeder“, „,die größte Primzahl“" widersinnig, weil die Gegenstände, die sie beschreiben wollen, nach den apriorischen Gesetzen ihrer jeweiligen Gegenstandsgebiete unmöglich sind; doch ihre Widersinnigkeit kann man aus ihren Bedeutungsformen nicht ablesen. Husserl bezeichnet derartigen Widersinn als materialen Widersinn oder synthetischen Widersinn. ${ }^{130}$

Im Gegensatz dazu lässt sich die Widersinnigkeit vom Ausdruck (2) schon aus seiner Bedeutungsform $\left[\left[Y_{\text {adj }} u n d\left[\text { nicht }-Y_{\text {adj }}\right]_{\text {adj }}\right]_{\text {adj }} X_{n}\right]_{n}$ ablesen; wir brauchen nicht den Gegenstand Viereck zu betrachten und Geometrie zu betreiben, um zu wissen, dass ein reguläres und zugleich nicht-reguläres Viereck unmöglich ist, oder mit Husserls eigenen Worten, dass der Ausdruck keine „,gegenständliche Geltung“ ${ }^{131}$ hat. Im Vergleich $\mathrm{zu}$ dem Terminus materialer Widersinn oder synthetischer Widersinn bezeichnet Husserl den Widersinn vom Ausdruck (2) als formalen oder analytischen Widersinn ${ }^{132}$. Besonders wenn ein formal-widersinniger Ausdruck ein Aussagesatz (Z.B. ,,alle Menschen sind sterblich und einige Menschen sind nicht sterblich“) ist, dann kann man schon an seiner Bedeutungsform erkennen, dass er falsch ist.

Die Geltungslehre der Bedeutungen beschäftigt sich mit Bedeutungsformen. Sie übernimmt die Aufgabe, die apriorischen Gesetze, die bestimmen, welche Formen eine konsistente Bedeutung tragen können, systematisch $\mathrm{zu}$ untersuchen, wobei die Schlußformen, in denen ein Urteil in Prämissenurteilen ,,analytisch“ beschlossen ist, eine ausgezeichnete Weise von Bedeutungskonsistenz (=Konsequenz) darstellt. ${ }^{133}$ Konsequenterweise übernimmt sie auch die Aufgabe, formale Konsistenz (insbesondere die formale Konsequenz) von formalem Widersinn zu unterscheiden. Die Aufgabe, materiale Konsistenz vom materialem Widersinn zu unterscheiden, sprengt

\footnotetext{
${ }^{130}$ Hua XIX/1: Logische Untersuchungen, S.343.

${ }^{131}$ Ebd., S.337.

132 Ebd., S.343.

${ }^{133}$ Hua XVII: Formale und transzendentale Logik, S.58, 68.
} 
schon den Rahmen einer formalen Untersuchung und gehört natürlich nicht zum Thema der Geltungslehre.

\section{§19. Unterschied und Zusammenhang der beiden Bedeutungslehren}

Nach den obigen Ausführungen sind die Formenlehre und die Geltungslehre in zwei Hinsichten ähnlich: (1) Beide Disziplinen erforschen die Bedeutungen; (2) beide Disziplinen beschränken die Forschung auf Bedeutungsformen. Aus diesem Grund fasst Husserl die beiden Disziplinen unter dem Titel reine Logik der Bedeutungen zusammen.

Doch die beiden Bedeutungslehren haben verschiedene Aufgaben und untersuchen verschiedenartige Gesetze von Bedeutungen. Der Ausdruck „rundes und nicht-rundes Viereck“ ist für die reine Grammatik zulässig. Denn nach der reinen Grammatik lässt sich eine adjektivische Bedeutung mit der Bedeutung „nicht“ zu einer neuen adjektivischen Bedeutung der Form [nicht- $\left.X_{\text {adj }}\right]_{\text {adj }}$ kombinieren; und zwei adjektivische Bedeutungen können sich durch die konjunktionale Bedeutung ,und“ zu einer umfassenderen adjektivischen Bedeutung $\left[\mathrm{X}_{\mathrm{adj}} \mathrm{und} \mathrm{Y}_{\mathrm{adj}}\right]_{\mathrm{adj}}$ zusammensetzen; schließlich vereint sich eine adjektivische Bedeutung mit einer substantivischen Bedeutung zu einer Bedeutung der Form $\left[\mathrm{X}_{\mathrm{adj}} \mathrm{Y}_{\mathrm{n}}\right]_{\mathrm{n}}$ - dann ergibt sich die gesetzmäßige Bedeutungsform $\left[\left[Y_{\text {adj }} u n d\left[\text { nicht }-Y_{\text {adj }}\right]_{\text {adj }}\right]_{\text {adj }} X_{n}\right]_{n}$, die durch die Bedeutung ,rundes und nicht-rundes Viereck“ materialisiert wird.

Allerdings ist die Bedeutungsform $\left[\left[Y_{\text {adj }} u n d\left[n i c h t-Y_{\text {adj }}\right]_{\text {adj }}\right]_{\text {adj }} X_{n}\right]_{n}$ für die Geltungslehre gesetzwidrig. Wie immer man die Buchstaben $\mathrm{X}$ und $\mathrm{Y}$ ausfüllt und somit die Form materialisiert, bekommt man unbedingt eine widersinnige Bedeutung, die keine gegenständliche Geltung haben kann. Denn diese Bedeutungsform verletzt das formale Gesetz vom ausgeschlossenen Widerspruch. Andere Beispiele von Gesetzen der Geltungslehre sind die Schlussregeln wie modus ponens, die Regeln des Syllogismus usw.

Danach ist ersichtlich: Dass eine Bedeutung die Gesetze der reinen Grammatik einhält, garantiert nicht, dass sie die Gesetze der Geltungslehre auch erfüllt. Es gibt 
Fälle, wo eine grammatisch gesetzmäßige Bedeutung konsistent ist; es gibt aber auch Fälle, wo sie widersinnig ist. Mit Husserls eigenen Worten, die Gesetze der Formenlehre „lassen es ganz offen, ob die in solchen Formen zu bildenden Bedeutungen ,gegenständlich“ sind oder ,gegenstandslos“, ob sie (wenn es sich um Satzform handelt) mögliche Wahrheit ergeben oder nicht“. ${ }^{134}$ Die beiden Bedeutungslehren behandeln verschiedenartige Bedeutungsgesetze.

Trotz der Verschiedenheit sind die beiden Disziplinen nicht beziehungslos. Man interessiert sich offensichtlich nicht für die formal-gegenständliche Geltung von Ausdrücken wie „König aber oder“. Denn sie tragen gar keine einheitliche Bedeutung, ganz zu schweigen von einer formal-konsistenten. Die Bedeutungen, zwischen denen die Geltungslehre Konsistenz und Widersinn zu unterscheiden versucht, müssen zuerst durch die Formenlehre gefiltert werden. Die Geltungslehre der Bedeutungen setzt also die Formenlehre der Bedeutungen voraus. Deshalb schreibt Husserl:

In der reinen Logik der Bedeutungen, deren höheres Ziel in Gesetzen gegenständlicher Geltung der Bedeutungen liegt, soweit solche Geltung durch die reine Bedeutungsform bedingt ist, bildet die Lehre vom Wesensbau der Bedeutungen und den Gesetzen ihrer Formenbildungen das notwendige Fundament. (Hua XIX/1, 337; meine Herv.)

Diese Gesetze des Sinnes [= Gesetze der Formenlehre - Anm. d. Verf.], normativ gewendet, des zu vermeidenden Unsinns, weisen der Logik die überhaupt möglichen Bedeutungsformen $\mathrm{zu}$, deren objektiven Wert sie allererst zu bestimmen hat. (a.a.O., 342-343)

Innerhalb der reinen Logik gibt es eine Sphäre von aller Gegenständlichkeit absehender Gesetze, die, im prägnanten Sinn, mit guten Gründen als reinlogisch grammatische zu bezeichnen wären. Noch besser stellen wir der reinen Formenlehre der Bedeutungen die sie voraussetzende reine Geltungslehre derselben gegenüber. (a.a.O., 302-303; meine Herv.)

Husserl gesteht, dass man allererst an die Gesetze der Geltungslehre denkt, wenn

\footnotetext{
${ }^{134}$ Hua XIX/1: Logische Untersuchungen, S.342.
} 
er von logischen Gesetzen redet. In diesem Sinne sind die Gesetze der Geltungslehre „die im prägnanten Sinn sogenannten logischen Gesetze“, während die Gesetze der Formenlehre ,im weiteren Wortsinn gewiß als formale logische Gesetze gelten müssen“. 135

Zusammmenfassend kann man sagen, (1) dass die Formenlehre und Geltungslehre als Teildisziplinen in die formale Logik der Bedeutungen einzuordnen sind; (2) dass sie verschiedenartige Bedeutungsgesetze untersuchen; (3) dass die Formenlehre ,das notwendige Fundament" für die Geltungslehre bildet.

In Formale und transzendentale Logik entwickelt Husserl eine umfassendere Idee der formalen Logik und somit eine neue Auffassung über das Verhältnis zwischen der reinen Grammatik und anderen logischen Disziplinen. Diese Entwicklung ist für uns auch interessant, denn sie ermöglicht eine Erweiterung der Rede vom Grammatikalitätsphänomen von der Bedeutungssphäre auf die Gegenstandssphäre. In folgenden Paragraphen versuche ich, Husserls neue Ausführungen darzustellen und damit die Rede des Grammatikalitätsphänomens von Gegenständen einzuführen.

\section{§20. Die Dreischichtung der formalen Logik von Bedeutungen}

In Formale und transzendentale Logik ist die Formenlehre weiterhin als eine Teildisziplin der formalen Logik von Bedeutungen zugeordnet - genauer gesagt, sie ist die unterste Stufe der formalen Logik von Bedeutungen. Doch Husserl spricht dabei nicht mehr von einer Zweischichtung von Formenlehre und Geltungslehre, sondern von einer Dreischichtung. Denn innerhalb der Geltungslehre macht er eine neue Arbeitsteilung: Die Geltungslehre differenziert sich in Konsequenzlogik der Bedeutungen und Wahrheitslogik der Bedeutungen. Die Unterscheidung zwischen den drei logischen Disziplinen lässt sich durch ihre jeweiligen Fragestellungen und Aufgaben erklären. ${ }^{136}$

Urteilsbedeutung (Proposition) spielt eine zentrale Rolle in der logischen Bedeutungslehre. Deshalb sind Bedeutungen, die selbst noch keine Gesamturteile sind,

\footnotetext{
135 Ebd.

${ }^{136}$ Hua XVII: Formale und transzendentale Logik, S.54-61.
} 
aber zu Urteilsgliedern eines Gesamturteils werden können (z.B. substantivische Bedeutung wie ,der Himmel“), in Formale und transzendentale Logik auch unter dem Titel Urteil (als Urteilsbedeutung) zusammengefasst. ${ }^{137}$ Daher ist in Formale und transzendentale Logik die Formenlehre der Bedeutungen auch als Formenlehre der Urteile bezeichnet. Ihre Hauptaufgabe ist, wie schon mehrmals erwähnt, zu untersuchen, welche Bedeutungen von welchen Bedeutungskategorien sich zu einer umfassenderen einheitlichen Bedeutung zusammensetzen können, oder normativ gewendet, Sinn vom Unsinn zu filtern und den letzteren zu vermeiden.

Allerdings sind manche von der Formenlehre anerkannte Ausdrücke, wie im letzten Paragraphen schon gezeigt, widersinnig. Konsistente Bedeutungen (insbesondere die ausgezeichneten Konsistenzformen des Beschlossenseins, d.i. der Konsequenz) von widersinnigen Bedeutungen in formaler Allgemeinheit zu unterscheiden, ist die Aufgabe einer anderen logischen Disziplin. In Fomale und transzendentale Logik bezeichnet Husserl diese andere Disziplin nicht mehr als Geltungslehre der Bedeutungen, sondern als Konsequenzlogik. Sie ist die zweite Stufe der formalen Logik der Bedeutungen.

Eine noch höhere Stufe der formalen Logik von Bedeutungen ist die sogenannte Wahrheitslogik. Ein Urteil ist in formaler Hinsicht konsistent, heißt nicht, dass es wahr ist oder sogar wahr sein kann. Nach der Konsequenzlogik ist das Urteil „Einige Dekaeder sind regulär“ eine formal-konsistente Urteilsbedeutung, da seine

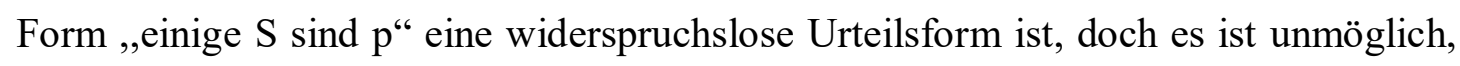
dass es wahr ist. Um solchen materialen Widersinn zu erkennen, muss man über die bloße Urteilsform hinausgehen. Mann muss also weiter fragen, ob die gegenständlichen Inhalte (hier die Eigenschaften der geometrischen Gegenstände) sachlich konsistent sind, d.i. keinen materialen Widersinn enthalten. Eine vollentwickelte Wahrheitslogik muss also die bloße formale Hinsicht der Urteile überschreiten, in diesem Sinn ist sie

\footnotetext{
137 Ebd., S.69: „Sie [= nicht-propositionale Bedeutungen] sind ... im weiteren Sinne auch „Urteile“, nur unselbständige, sofern sie in der Erkenntniseinstellung dazu bestimmt sind, zu Urteilsgliedern apophanticher Ganzheiten (der Urteile im prägnanten Sinne) zu werden, und nur so Erkenntnisbedeutung gewinnnen. Auch diese Urteile des gegenüber dem üblichen erweiterten Sinnes - ein Urteilsbegriff, den wir hinfort festhalten werden stehen in den oben bezeichneten analytischen Grundverhältnissen [d.i. Konsequenz (Beschlossensein), Konsistenz (leere Widerspruchlosigkeit), Inkonsequenz (Ausgeschlossensein)].“
} 
nicht mehr eine bloße formal-logische Disziplin. Dabei stellt sich die Frage, wieweit kann man im Rahmen der formalen Logik die Wahrheitslogik betreiben? Dazu schreibt Husserl:

Es ist sofort sichtlich, dass Widerspruchslosigkeit eine Wesensbedingung möglicher Wahrheit ist, dass aber erst durch einen wesensgesetzlichen und in einer Logik eigens $\mathrm{zu}$ formulierenden Zusammenhang dieser an sich zu unterscheidenden Begriffe sich die bloße Analytik in eine formale Wahrheitslogik verwandelt. ${ }^{138}$

Obwohl Konsistenz eines Urteils seine Wahrheit nicht garantiert, doch formale Konsistenz ist mindestens die notwendige Bedingung dafür, dass es wahr ist oder mindestens wahr sein kann. ${ }^{139}$ Aus diesem Grund lassen sich die logischen Gesetze über Urteilskonsistenz in Gesetze über das mögliche Wahrsein des Urteils verwandeln. Dafür bietet Husserl die beiden Lesarten des Widerspruchsgesetzes als ein Beispiel ${ }^{140}$ :

(Das Widerspruchsgesetz in Lesart der Konsequenzlogik)

Von zwei kontradiktorischen (und somit nicht-konsistenten) Urteilen sind nicht beide als eigentliche Urteile möglich, d.i. nicht beide zur Evidenz der Deutlichkeit ${ }^{141}$ zu bringen; nur eines davon ist zur Evidenz der Deutlichkeit zu bringen. Und die Konjunktion von beiden kann nicht als ein deutliches Urteil gelten.

(Das Widerspruchsgesetz in Lesart der Wahrheitslogik)

Von zwei nicht-konsistenten und sogar kontradiktorischen Urteilen können nicht beide wahr sein, nur eines davon ist wahr, während das andere falsch ist.

Und die Konjunktion von beiden kann nicht wahr sein.

Formaler Widerspruch/Konsistenz ist Grundbegriff der Konsequenzlogik. Die beiden Lesarten des Widerspruchsgesetzes benutzen diesen Begriff jeweils zu verschiedenen Themen (Konsistenz und Wahrheit der Urteile). Husserl zufolge ist die zweite Lesart eine Umformulierung der ersten, denn formale Konsistenz ist eine

\footnotetext{
${ }^{138}$ Hua XVII: Formale und transzendentale Logik, S.60-61.

${ }^{139}$ Ebd., S.60-61,70-71.

140 Ebd., S.71-72.

${ }^{141}$ Den Begriff ,,die Evidenz der Deutlichkeit “ werde ich im nächsten Paragraphen erläutern. Zur vorläufigen Verständigung sagt diese Lesart, dass ein rationaler Urteilender kein inkonsistentes Urteil erzeugt.
} 
notwendige Bedingung für die Wahrheit. Die Umformulierung verläuft wie folgt: (1) Nur deutliche Urteile können wahr sein; (2) Urteile, die einen formalen Widerspruch enthalten, können nicht deutlich sein; (3) deshalb können Urteile, die einen formalen Widerspruch enthalten, nicht wahr sein. Ähnliches gilt auch für andere Gesetze der Konsequenzlogik(wie modus ponens und tollens ${ }^{142}$ ). In diesem Sinne ist die formale Wahrheitslogik eine Umformulierung der Konsequenzlogik. Dieter Lohmar merkt an: „Solange die Wahrheitslogik formal bleibt, ist sie auf die Verwandlung konsequenzlogischer Prinzipien beschränkt. “143

Zusammenfassend kann man sagen, dass die formale Logik der Urteile drei Stufen hat, nämlich Formenlehre der Bedeutungen, Konsequenzlogik sowie Wahrheitslogik. Die Formenlehre liefert der Konsequenzlogik die Kandidaten (sinnvolle Urteile), zwischen denen die Konsequenzlogik die konsistenten aussucht. Dann bildet die Konsequenzlogik das Fundament für die Wahrheitslogik und wird zu ihrem Grundstück, und zwar durch den wesentlichen Zusammenhang, dass die formale Konsistenz die notwendige Bedingung für die Wahrheit ist.

\section{§21. Evidenzarten des Urteils}

Husserl zufolge kann man die Weisen, in denen die Urteile (als Bedeutungen) dem urteilenden Subjekt gegeben werden, unterscheiden. Diese „subjektiven Gegebenheitsweisen“" 144 nennt Husserl auch Evidenzarten der Urteile ${ }^{145}$. Die Unterscheidung dieser Evidenzarten begründet, warum die formale Logik sich in drei Schichten differenziert - die drei Schichten der formalen Logik erforschen Urteile jeweils unter Betonung der jeweiligen Evidenzarten. Man kann sagen, dass sich die Dreischichtung der formalen Logik durch drei Evidenzarten des Urteils subjektiv begründen lässt. ${ }^{146}$ Der Dreischichtung der formalen Logik entsprechend gibt drei

\footnotetext{
${ }^{142}$ Vgl. Hua XVII: Formale und transzendentale Logik, S.72.

${ }^{143}$ Dieter Lohmar, Edmund Husserls ,Formale und transzendentale Logik“, Wissenschaftliche Buchgesellschaft, Darmstadt 2000, S.59.

144 Hua XVII: Formale und transzendentale Logik, S.61.

${ }^{145}$ Die Selbstgegebenheit des Urteils (als Bedeutung) muss man von der der Gegenständilichkeit unterscheiden. Bei einem bloß signitiven Urteilsakt kann das Urteil (als Bedeutung) schon originaliter selbstgeben sein, während die intendierte Gegenständlichkeit es nicht ist.

${ }^{146}$ Hua XVII, S.72: „Bei der bloßen Vorzeichnung der in einer formalen Logik notwendig vorzunehmenden
} 
Evidenzarten: die Evidenz der Verworrenheit, die der Deutlichkeit und die der Klarheit.

\section{a) Evidenz der Verworrenheit}

In den Fällen, wo das Subjekt einen vagen Einfall hat, oder wo er beim Hören oder Lesen der Wortfolge eines komplizierten Satzes folgt, ohne den Satz im wirklichen Denken zu artikulieren, hat er eigentlich kein Urteil erzeugt. In solchen Fällen ist eine Bedeutungseinheit durch den sprachlichen Ausdruck passiv indiziert, aber das Subjekt urteilt eigentlich nicht. ${ }^{147}$ Wenn ich z.B. einen komplizierten und langen Satz in einem theoretischen Textbuch lese, kann es passieren: Ich habe die Wortfolge des Satzes aufmerksam durchlaufen und bin sicher, dass es sich dabei um einen grammatisch wohlgeformten Satz handelt, aber ich verstehe den Satz nicht und bin nicht in der Lage, ihn ,eigentlich“ zu urteilen, d.i. ihn mit Überzeugung zu urteilen. ${ }^{148}$ Laut Husserl wird in diesem Fall ein Urteil in Evidenz der Verworrenheit gegeben oder indiziert.

Ein inkonsistentes Urteil der Satzform ,,S ist p und nicht p“kann auch in Evidenz der Verworrenheit gegeben werden, aber niemals in einem eigentlichen Urteilsakt, denn kein rationaler Urteilender wird ein kontradiktorisches Urteil fällen. Vielleicht spricht ein Sprecher eine Aussage der Form „S ist $\mathrm{p}$ und nicht $\mathrm{p}$ “ aus, aber er ist dabei nur ein Sprecher und scheinbarer Urteilender - offensichtlich will er nicht so urteilen. Auch in dem Fall, wo ein Sprecher eine komplizierte kontradiktorische Aussage ernstlich ausspricht, deren impliziter Widerspruch ihm unbewusst ist, gilt er nicht als ein eigentlicher Urteilender - Sokolowski würde sagen, dass das Subjekt hier nur in einer degenerierten Weise (degenerate way) ${ }^{149}$ denkt und urteilt. Streng genommen ist hier kein Urteil im eigentlichen Sinne gegeben, sondern es wird nur ein wohlgeformter Ausdruck konstituiert. ${ }^{150}$ Husserl zufolge lassen sich alle Urteilsbedeutungen, die den

Scheidungen ... kann es nicht sein Bewenden haben. Es bedarf tiefer dringender, die entsprechend unterschiedenen Evidenzen auslegender Begründungen, mit denen auch erst eine wirkliche Einsicht in die Notwendigkeit und Tragweite dieser Scheidungen sich eröffnen kann.“

${ }^{147}$ Vgl. ebd., S.61-63.

${ }^{148}$ Vgl. Robert Sokolowski, Husserlian Meditations. How Words present Things, Northwestern Universtiy Press, Evanston 1974, S.211: „It is possible for a judgment to be only confusedly or vaguely executed ... Passive reading or listening, or verbal formulations that we let ourselves make without thinking, are examples; we let the expression come about, and it may be perfectly distinct phonemically and correct grammaticallly, but we do not frame the judgment. We do not put its parts together explicitly.“

${ }^{149}$ Ebd.

${ }^{150}$ Vgl. Robert Sokolowski, Introduction to Phenomenology, Cambridge University Press, Cambridge 2000, S.105ff. 
Gesetzen der Formenlehre gehorchen, in Evidenz der Verworrenheit erzeugen ${ }^{151}$.

\section{b) Evidenz der Deutlichkeit}

Wenn ein Urteil im Urteilsakt eigentlich geurteilt wird, d.i. dass das Subjekt die Urteilsbedeutung verstehend und mit Überzeugung erzeugt ${ }^{152}$, dann ist es in Evidenz der Deutlichkeit gegeben. Eine notwendige Bedingung für die Deutlichkeitsevidenz ist, dass die Form des Urteils konsistent ist. ${ }^{153}$

Es gibt Fälle, wo das Urteil in einer gemischten Evidenzart erzeugt wird. Z.B. beim Lesen eines implizit Widerspruch enthaltenden Satzes wird das urteilende Subjekt manche Glieder des Urteils in Deutlichkeit urteilen, während die Glieder, die gegenseitig widerstreiten, nur in Verworrenheit erzeugt werden. ${ }^{154}$

In manchen Fällen wird ein Urteil zuerst in Verworrenheit gegeben, dann durch den expliziten Urteilsvollzug in Deutlichkeit als dasselbe Urteil verwandelt. Hier findet eine Erfüllungssynthese desselben Urteils statt - das Urteil ist zuerst nur indiziert, dann originaliter gegeben. In diesem Sinne ist die Evidenz der Deutlichkeit eine Erfüllung der Evidenz der Verworrenheit.

Wie steht es mit den Fällen, wo es dem Subjekt nach dem Versuch, eine zuerst verworrene Urteilsbedeutung verstehend zu erzeugen (= artikulieren), bewusst wird, dass das artikulierte Urteil, das vorher in Verworrenheit gegeben ist, einen Widerspruch beinhaltet? Dabei wird das verworrene Urteil in gewissem Sinne auch verdeutlicht, aber nicht in Evidenz der Deutlichkeit erzeugt und nicht eigentlich geurteilt. Nach der Artikulation erfährt hier das Urteil die Modifikation der Durchstreichung, und der Urteilende wird natürlich kein solches Urteil fällen. Das nenne ich den Fall der negativen Verdeutlichung, im Vergleich dazu ist die Verdeutlichung, die durch die Verwandlung des verworrenen Urteils in Deutlichkeit vollzogen wird, als positive

\footnotetext{
${ }^{151}$ Die Rede von Erzeugen lässt sich im Rahmen der transzendentalen Phänomenologie als Konstitution verstehen. Dazu vgl. unten Kapitel II und III, im II. Abschnitt der vorliegenden Arbeit.

${ }^{152} \mathrm{Im}$ Vergleich zu der für Sokolowski degenerierten Weise des Urteilens.

${ }^{153}$ Grob gesagt ist ein Urteil dann deutlich vollziehbar, wenn ein rationales Subjekt es ernsthaft fällen kann. Ein formal inkonsistentes Urteil wie „Alle Menschen sind sterblich und einige Menschen sind nicht sterblich“ kann ein rationales Subjekt nicht mit Überzeugung fällen. Außerdem können auch Urteile, die Kategoriefehler begehen (wie „Die Gerechtigkeit ist blau“), nicht in Evidenz der Deutlichkeit gegeben werden.

${ }^{154}$ Hua XVII: Formale und transzendentale Logik, S.65.
} 
Verdeutlichung zu bezeichnen. ${ }^{155}$ Ein Beispiel der negativen Verdeutlichung: Ich begegne einem Satz der etwas komplizierten (und kontradiktorischen)

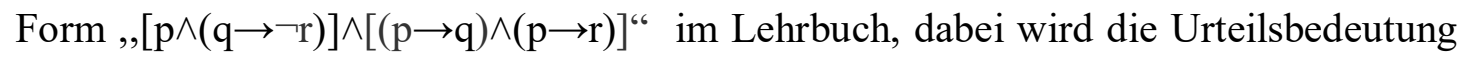
(Proposition) des Satzes nomalerweise zunächst in Evidenz der Verworrenheit gegeben - ich weiß, dass der etwas komplizierte Satz grammatisch wohlgeformt ist und eine einheitliche Proposition ausdrückt, nur dass ich mich noch anstrengen muss, diese Proposition genauer zu artikulieren und verstehen; ich versuche, den Satz mehrmals zu lesen und die Proposition zu artikulieren und verstehen, dann fällt es mir ein, dass die

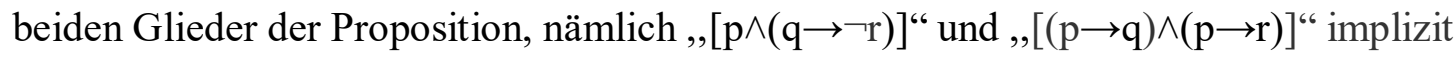
sich einander widersprechen, was mir auf den ersten Blick des Satzes unmerklich war! In diesem Fall habe ich das Urteil (Proposition) zwar verdeutlicht, aber nur im negativen Modus - ich verstehe das Urteil, ohne es eigentlich zu fällen (= in Evidenz der Deutlichkeit zu bringen).

Eine negative Verdeutlichung kann stattfinden, auch wenn das artikulierte Urteil konsistent ist. Nach der Artikulation kann sich ein konsistentes Urteil (Proposition) ergeben, dem das Subjekt nicht zustimmt. Wenn man einem früher verworrenen, nun aber verdeutlichten Urteil (Proposition) nicht zustimmt, dann will er es natürlich nicht eigentlich fällen (= in Evidenz der Deutlichkeit bringen). Aber das, dass ein Urteilender eine Proposition ableugnet, heißt beim weitem nicht, dass es unmöglich ist, diese Proposition im Urteilsakt eigentlich zu erzeugen, sonst würde es gar keine Meinungsverschiedenheit in der menschlichen Gesellschaft geben. Die Umgrenzung des Gebietes ,„deutliche Urteile“ hängt also nicht von dem tatsächlichen Zustimmen oder Ablehnen der Subjekte ab, sondern nur davon, ob es möglich ist, dass ein Subjekt das Urteil eigentlich fällt. ${ }^{156}$ Die formale Konsistenz des Urteils ist formale Voraussetzung für diese Möglichkeit.

Eine Aufgabe der Konsequenzlogik ist, wie mehrmals gesagt, konsistente Urteile von inkonsisten zu filtern; danach untersucht sie die logischen Verhältnisse zwischen

\footnotetext{
155 Ebd., S.63-64.

156 Ebd., S.67-68.
} 
den ausgesuchten konsistenten Urteilen. ${ }^{157} \mathrm{Da}$ formale Konsistenz die formale Voraussetzung der Urteilsdeutlichkeit ist, bilden die deutlichen Urteile das zentrale Forschungsgebiet für die Konsequenzlogik. „So hat der rein analytische Logiker die Wesensgattung deutliches Urteil mit ihrem Umfang möglicher Urteile als sein Gebiet.“158 Das heißt, die Urteile, die in Evidenz der Deutlichkeit gegeben werden können, bilden das thematische Forschungsgebiet der Konsequenzlogik.

\section{c) Evidenz der Klarheit}

Ein Urteil in Evidenz der Deutlichkeit erzeugen, heißt nicht, dass das Urteil schon evident wahr ist. Ich urteile, dass die Katze auf dem Schreibtisch liegt - dabei wird ein deutliches Urteil gegeben. Aber das Urteil bleibt im Modus der Deutlichkeit und ist noch nicht evident wahr, bis ich selber wahrnehme, dass die Katze auf dem Tisch liegt. Ein Urteil, das aufgrund der Anschauung des entsprechenden Sachverhalts erzeugt ist, nennt Husserl als ein klares Urteil. Seine Evidenzart ist die Evidenz der Klarheit ${ }^{159}$. Husserl schreibt:

Doch hier verzweigen sich noch die Unterschiede, insofern als die „Klarheit“ bezeichnen kann das Urteilen in dem Modus des seinen vermeinten Sachverhalt selbst gebenden, also das, was man gewöhnlich als evidentes Urteilen im Auge hat, und wieder das Urteilen im Modus eines sich den vermeinten Sachverhalt vor-verbildlichenden, anschaulich machenden Urteilens. ${ }^{160}$

Ein Urteil ist evident oder klar, wenn sein entsprechender Sachverhalt in der Anschauung gegeben ist (in der Wahrnehmung selbstgegeben oder mindestens in der Phantasie verbildlicht). Nicht alle deutlichen Urteile lassen sich tatsächlich durch

\footnotetext{
${ }^{157}$ Dies sind z.B. Konsequenz (Beschlossensein), Konsistenz (leere Widerspruchlosigkeit) und Inkonsequenz (Ausgeschlossensein). Darüber vgl. Hua XVII: Formale und transzendentale Logik, S.68-69.

${ }^{158}$ Hua XVII: Formale und transzendentale Logik, S. 68.

${ }^{159}$ Der Begriff von Klarheitsevidenz ist verträglich mit dem Evidenzbegriff im [§39] der VI. LU. Dort ist Evidenz ein Erlebnis der Übereinstimmung des im Urteilen Gemeinten und des in Anschauung Gegebenen. Das passiert auch im Fall des klaren Urteilens. Meines Erachtens ist dieses Erlebnis der Übereinstimmung (als Acquaintance zweiter Stufe) sogar wesentlich für die Klarheitsevidenz des betreffenden Urteils, denn Urteil in Klarheitsevidenz ist durch Anschauung erfüllt.

${ }^{160}$ Ebd., S. 66.
} 
entsprechende Wahrnehmungen in die Klarheit der Selbsthabe überführen. Z.B. ist das Urteil ,,die Erde ist ein Würfel“ tatsächlich durch die Satellitenbeobachtung negiert. Allerdings kann es in einer Verbildlichung erfüllt werden, mit Husserls eigenen Worten, es kann in Klarheit der Antizipation gegeben werden. Wenn ein Urteil in Klarheit der Antizipation erzeugt werden kann, dann ist es möglicherweise wahr, auch wenn es tatsächlich nicht wahr ist. Im Vergleich dazu ist das Urteil „,die Erde ist ein reguläres Dekaeder"weder durch Erfahrung im Modus der Selbsthabe noch durch Verbildlichung erfüllbar, da es gar kein reguläres Dekaeder gibt. Und es ist unmöglich, dass es wahr ist.

Die Wahrheitslogik behandelt eben die Urteile, die mögliche Wahrheit haben, deshalb bilden ihr zentrales Forschungsgebiet die Urteile, die in Klarheit der Selbsthabe oder in Klarheit der Antizipation erzeugt werden können. Da der Umfang der ersteren in dem der letzteren eingeschlossen ist, kann man einfach sagen, das zentrales Forschungsgebiet der Wahrheitslogik sind die Urteile, die in Evidenz der Klarheit (der Antizipation) erzeugt werden können.

Die drei Evidenzarten des Urteils entsprechen also den drei Schichten der formalen Logik von Bedeutungen (bzw. der formalen Apophantik). Die Urteile, die mindestens in Evidenz der Verworrenheit erzeugt werden können, bilden das Hauptgebiet für Formenlehre, die deutlichen Urteile für Konsequenzlogik, und die klaren Urteile für Wahrheitslogik. Die formale Logik, die die Urteilsbedeutungen untersucht, hat drei Stufen, weil die Urteile in drei verschiedenen subjektiven Evidenzarten gegeben sind. Aus diesem Grund betrachtet Husserl die Unterscheidung der Evidenzarten von Urteil als subjektive Begründung der Dreischichtung von formaler Apophantik. ${ }^{161}$

\section{§22. Der Unterschied und Zusammenhang zwischen formaler Apophantik und formaler Ontologie}

Bisher haben wir das Forschungsgebiet der formalen Logik auf die Urteile (Bedeutungen) beschränkt, unter solcher Beschränkung ist die formale Logik auch als

\footnotetext{
${ }^{161}$ Ebd., S.61.
} 
formale Apophantik bezeichnet. Doch die formale Logik im vollen Sinne muss nicht nur die Formen der Bedeutungen, sondern auch die der bedeuteten Gegenstände betrachten. Schon der obige Übergang von Konsequenzlogik zu Wahrheitslogik deutet darauf hin, dass die formale Logik die Gegenstände in Betracht ziehen muss, denn Wahrheit heißt, dass das Urteil zur „Adäquation an die Sachen selbst“"162 gebracht werden kann.

Husserl unterscheidet nicht nur kategoriale Formen der Bedeutung wie Begriff, Urteil, Schluss usw., sondern auch kategoriale Formen der Gegenständlichkeit wie Gegenstand, Sachverhalt, Relation, Anzahl, Menge usw. Die erstere Gruppe bezeichnet Husserl als Gruppe der Bedeutungskategorien, die letztere Gruppe ist als die der Gegenstandskategorien zu bezeichnen. ${ }^{163}$ Die formale Logik als Apophantik untersucht die Bedeutungkategorien und deren formale Gesetze. Husserl zufolge hat die traditionelle Logik seit Aristoteles diese Aufgabe schon zum Teil durchgeführt. Andererseits sind die Gegenstandskategorien und deren formale Gesetze das Thema einer anderen formalen Wissenschaft, nämlich der formalen Ontologie. ${ }^{164}$ Die historischen formal-mathematischen Disziplinen haben zu der formal-ontologischen Untersuchung beigetragen, z.B. untersucht die Mengenlehre die Gegenstandskategorie Menge, die Arithemetik die Anzahl usw. ${ }^{165}$ Individuum, Menge, Relation, usw. sind gegenständliche Formen, sie sind die Ableitungsgestalten des höchsten Formbegriffs ,etwas-überhaupt“ und lassen sich in formaler Allgemeinheit auf alle besonderen Gegenstandsgebiete anwenden. Aus diesem Grund scheint die formale Mathematik eben die formale Ontologie zu sein. Husserl schreibt:

Hieraus erwächst eine universale Wissenschaftsidee, die einer formalen Mathematik im voll umfassenden Sinne, deren Universalgebiet sich fest umgrenzt als Umfang des obersten Formbegriffs Gegenstand-überhaupt oder

\footnotetext{
${ }^{162}$ Husserl vertritt hier eine Evidenztheorie der Wahrheit, nach der jedes wahre Urteil (Proposition) im Prinzip aufgrund einer adäquat erfüllenden Anschauung erzeugt werden kann. Doch Wolfgang Künnes Argument gegen Putnams „epistemische Wahrheitsauffassung“ zeigt, dass Husserls Evidenztheorie problematisch ist. Dazu vgl. Wolfgang Künne, „Bolzanos blühender Baum - Plädoyer für eine nicht-epistemische Wahrheitsauffassung“, in: Realismus und Antirealismus, hrsg. von Forum für Philosophie Bad Homburg, Frankfurt am Main: Suhrkamp 1992, S.224-244.

${ }^{163}$ Hua XVII: Formale und transzendentale Logik, S.92.

${ }^{164}$ Ebd., S.81.

${ }^{165}$ Es ist zu bermerken, für Husserl ist die Geometrie keine formale Mathematik, sondern materiale Mathematik.
} 
des in leerster Allgemeinheit gedachten Etwas-überhaupt, mit allen in diesem Feld apriori erzeugbaren und daher erdenkbaren Ableitungsgestalten, die in immer neuer iterativer Konstruktion immer neue Gestalten ergeben. Solche Ableitungen sind neben Menge und Anzahl (endliche und unendliche), Kombination, Relation, Reihe, Verbindung, Ganzes und Teil usw. So liegt es nahe, diese ganze Mathematik als eine Ontologie (apriorische Gegenstandslehre), aber als eine formale, auf die reinen Modi des Etwasüberhaupt bezogene anzusehen. (Hua XVII, 82)

Normalerweise sind formale Apophantik und formale Mathematik als verschiedene Wissenschaften angesehen, getrennt durch ihre jeweiligen Gebiete von Bedeutungskategorien und Gegenstandskategorien. Doch Husserl ist der Meinung, „dass formale Ontologie und formale Apophantik trotz ihrer ausdrücklich verschiedenen Thematik doch sehr nahe zusammengehören müssen und vielleicht untrennbar sind.“(a.a.O., 83) Dafür hat Husserl zwei Argumente dargeboten:

(1) Alle Gegenstandsformen können nur in Urteilsakten konstituiert werden. Husserl schreibt:

Schließlich treten doch alle Formen von Gegenständen, alle Abwandlungsgestalten des Etwas-überhaupt in der formalen Apophantik selbst auf, wie ja wesensmäßig Beschaffenheiten (Eigenschaften und relative Bestimmungen), Sachverhalte, Verbindungen, Beziehungen, Ganze und Teile, Mengen, Anzahlen und welche Modi der Gegenständlichkeit sonst, in concreto und ursprünglich expliziert, für uns als wahrhaft seiende oder möglicherweise seiende nur sind als in Urteilen auftretende...Im pluralen Urteil z.B. kommt ja der Plural vor, im allgemeinen Urteil das Allgemeine. (a.a.O.)

Dem Zitat zufolge besteht also ein Parallelismus zwischen Bedeutungsformen und Gegenstandsformen: der Bedeutungsform Proposition entspricht die Gegenstandsform Sachverhalt, der Bedeutungsform Plural entspricht die Gegenstandsform plurale Gegenständlichkeit usw. Wegen dieses Parallelismus ,liegen in allen formalen Urteilsunterscheidungen auch Unterschiede der Gegenstandsformen mitbeschlossen.“ (a.a.O.) Husserl beschränkt den Umfang der Urteilsakte nicht auf die 
bevorzugten prädikativen Urteilsakte. ${ }^{166}$ „Freilich sind in dieser Betrachtung Aktivitäten wie Kolligieren, Zählen, Ordnen, Kombinieren usw. den Urteilsaktivitäten zugerechnet worden und ihre Korrelate den Urteilsgebilden.“(a.a.O., 112) MengeBedeutungen sind Gebilde der kolligerenden Urteilsaktivitäten, ganz wie die Sachverhalt-Bedeutungen (Urteile im prägnanten Sinne) Gebilde der prädikativen Urteilsaktivitäten sind.

(2) Syntaktische Akte wie Zählen, Kolligieren ${ }^{167}$ sind nicht prädikative Urteilsakte, deren Gebilde als Urteile im prägnanten Sinne in der Apophantik bevorzugt sind. Doch die Gebilde von diesen anderen syntaktischen Akten, wie eben gezeigt, haben einerseits ihre Vertreter im prädikativen Urteil und lassen sich durch erfolgende Nominalisierung zum Gegenstand-worüber verwandeln. ,,So gut Eigenschaft eine im Urteil zunächst unselbständig auftretende Form bezeichnet, die „,nominalisiert“ die Substratform Eigenschaft ergibt, so tritt im pluralen Urteilen der Plural auf, der „,nominalisiert“, zum Gegenstand im ausgezeichneten Sinne umgestaltet - dem des Substrates, des „Gegenstandes-worüber“ - die Menge ergibt.“ (a.a.O., 113) Andererseits ist es immer möglich, dass die Gebilde von anderen syntaktischen Akten in die des prädikativen Urteilens eintreten und als Komponenten fungieren. Besonders im Erkenntnisstreben dienen die anderen syntaktischen Akte nur als Mittel der prädikativen Urteilsakte. ,Z.B. man kolligiert und zählt dann nicht zum Spiel, oder weil man daran aus welchen Gründen sonst interessiert ist, sondern im Interesse der Erkenntnis des Gebietes (z. B. der Natur), letztlich also um die betreffenden Elemente und Einheiten als ihm zugehörige zu erkennen und prädikativ (apophantisch) zu bestimmen.“( (a.a.O., 114) Aus diesen Gründen muss die Apophantik nicht nur die

\footnotetext{
${ }^{166} \mathrm{Vgl}$. den Begriff der propositionalen Akte im [§4] der vorliegenden Arbeit.

${ }^{167}$ Hintergrund: In welchem Sinn zählt Husserl auch das Kolligieren und Zählen zu syntaktischen Akten? In Formale und transzendentale Logik (\$39) verweist Husserl, als er Kolligieren und Zählen erwähnt, auf sein Frühwerk Philosophie der Arithemetik, wo er versucht, den psychologischen Ursprung von Vielheit- (=Menge) und Anzahlbegriff aufzuzeigen. Dabei betrachtet er, grob gesagt, Kolligieren und Zählen als zwei Seiten derselben Münze - das kollektive Meinen (wie „John und Smith und Bill“). Das kollektive Meinen ist kein bloßes Nebeneinander- oder Nacheinander-Meinen der Einzelobjekte, sondern vereinigt sie zu einer Einheit (,John und Smith und Bill sind ein gutes Team"), in diesem Sinne ist das kollektive Meinen ein syntaktischer Akt. Husserl zufolge kann man aufgrund des kollektiven Meinens von den besonderen Merkmalen der kolligierten Einzelobjekte absehen und bekommt eine leere Vielheit wie „etwas und etwas und etwas“ - das ist der psychologische Ursprung des formalen Vielheitsbegriffs; zugleich unterscheidet man zwischen verschiedenen leeren Vielheiten (z.B. ,,etwas und etwas" (=2) vs. „etwas und etwas und etwas“ $(=3)$ ), daraus entstehen eine Reihe von Vielheiten, die den psychologischen Ursprung von Anzahlreihe ausmachen. Näheres darüber vgl. Hua XII: Philosophie der Arithmetik, S.79-82, 131.
} 
Gebilde des prädikativen Urteilsaktes, sondern auch die des Zählens, Kolligierens usw. betrachten, wobei die prädikativen Urteile ihr zentrales Thema bilden.

In beiden Argumenten spricht Husserl von Gebilden der Urteilsaktivitäten im weiteren Sinne; unter dem Begriff Gebilde versteht er nicht die kategoriale Gegenständlichkeit (Sachverhalt, Menge, usw.), die der Urteilende geradehin intendiert, sondern die Bedeutung (Sachverhalt-Bedeutung, Menge-Bedeutung), die sich auf die kategoriale Gegenständlichkeit bezieht. Nach Argument (1) sind die Gebilde des formal-mathematischen Denkens Menge-Bedeutungen, Anzahl-Bedeutungen usw., und die formale Mathematik (eine Zweideutigkeit und ein scheinbarer Widerspruch finden hier statt!) ${ }^{168}$ untersucht eben diese Gebilde; nach Argument (2) sind die Gebilde des mathematischen-Denkens mögliche Komponenten der Gebilde vom prädikativen Urteilen, deshalb ist die formale Mathematik sogar als eine Abteilung der Apophantik zugerechnet. Danach ist eine Zusammengehörigkeit der formalen Apophantik und formalen Mathematik begründet.

Diese Überlegung scheint dem, was wir am Anfang des Paragraphen zeigen wollten, zu widerstreiten. Am Anfang wollten wir zeigen, dass die formale Mathematik die Gegenstandskategorien, die von den Bedeutungskategorien zu unterscheiden sind, untersucht, damit die formale Mathematik den Namen der formalen Ontologie verdient. Wir wollten auch zeigen, dass die formale Mathematik als formale Ontologie verschieden ist von der formalen Apophantik. Wie können wir nun sagen, dass die formale Mathematik die Bedeutungsgebilde des mathematischen Denkens untersucht und somit einen Teil der Apophantik bildet? Ist das nicht ein Widerspruch?

Husserl zufolge ist das nur ein scheinbarer Widerspruch, denn die Rede von Mathematik ist hier zweideutig. Wir werden diese Zweideutigkeit im [§24] erklären. Aber bevor wir darüber ausführlich diskutieren, betrachten wir zuerst die subjektive Begründung für den regionalen Unterschied zwischen Bedeutung und Gegenständlichkeit, der für das Verständnis der Unterscheidung von

\footnotetext{
${ }^{168}$ Hier findet die Zweideutigkeit der Rede von formaler Mathematik statt. Bei der Rede von formaler Mathematik handelt es sich entweder um die Mathematik im logischen Sinne, die als formale Ontologie fungiert, oder um die reine Mathematik, die nur eine Abteilung der formalen Apophantik bildet. Die beiden Argumente gelten nur für die reine Mathematik. Darüber mehr im [\$24] der vorliegenden Arbeit.
} 

notwendig ist.

\section{§23. Die subjektive Begründung für den Unterschied zwischen formaler}

\section{Apophantik und Ontologie}

Ganz wie die Dreischichtung der Apophantik eine subjektive Begründung ${ }^{169}$ erfordert, die die drei Evidenzarten (Gegenbenheitsweisen) des Urteils unterscheidet und somit rechtfertigt, warum die formale Apophantik sich in drei Schichten differenziert (weil Urteile drei Gegebenheitsweisen haben), gilt es auch für die Zweigliederung der formalen Logik in Apophantik und Ontologie, die jeweiligen Evidenzarten (Gegenbenheitsweisen) der Bedeutungen und Gegenstände zu unterscheiden und somit zu rechtfertigen, dass die formale Logik sich in die beiden Disziplinen differenzieren soll (weil Bedeutungen und Gegenstände in verschiedenen Weisen gegeben werden). ${ }^{170}$ In 2. Teil des 1. Abschnitts der Formalen und transzendentalen Logik setzt Husserl sich eben mit den Weisen auseinander, wie Bedeutungen und Gegenstände zum Thema des urteilenden Subjekts werden. Das ZumThema-Werden der Gegenstände und Bedeutungen geschieht jeweils in der geradehin urteilenden Einstellung bzw. der kritischen Einstellung, nun diskutieren wir, was die beiden Einstellungen sind und wie sie sich zueinander verhalten.

\section{a) Die geradehin urteilende Einstellung}

Alltägliche Urteilsakte sind hauptsächlich Urteilsakte über Gegenstände, die das urteilende Subjekt für wirklich hält und bestimmen will. Z.B. wenn man das Urteil ,Das Auto ist rot“ fällt, dann ist er intentional auf das Auto, das er für wirklich-seiend hält,

\footnotetext{
${ }^{169}$ Die subjektive Begründung ist eine Art Konstitutionsanalyse (vgl. unten II. und III. Kapitel im II. Abschnitt der vorliegenden Arbeit), die versucht zu zeigen, dass die Verschiedenheit des „Themas“ der intentionalen Akte auf die Verschiedenheit der entsprechenden Bewußtseinsweisen zurückzuführen ist. Die subjektive Begründung der Dreischichtung der Apophantik ist zu zeigen, wie schon erörtert, dass Urteile auf drei Weisen im Bewußtsein konstituiert werden können. In analoger Weise versucht die subjektive Begründung der Zweigliederung der Logik in Ontologie und Apophantik zu zeigen, dass formale Ontologie und Apophantik jeweils zwei Bewußtseinseinstellungen entsprechen. In den beiden Einstellungen sind jeweils Gegenstände schlechthin und Bedeutungen thematisiert.

${ }^{170}$ Hua XVII: Formale und transzendentale Logik, S.116.
} 
gerichtet. Das Auto ist sein Gegenstand-worüber. In einem zweiten Sinn ist er auf die Bestimmung (das Rotsein) des Gegenstands-worüber und ferner auf den ganzen Sachverhalt, dass das Auto rot ist, gerichtet - alle gehören zum Ziel des Gerichtetseins. ${ }^{171}$ Was besonders beim geraden Urteilen außer Betracht bleibt, ist die Urteilsbedeutung. Husserl schreibt:

Urteilend sind wir nicht auf das Urteil, sondern auf die jeweiligen „Gegenstände-worüber“ (Substratgegenstände), auf die jeweiligen Prädikate, das ist gegenständlich bestimmenden Momente, auf die Relationen, in kausalen Urteilen auf die jeweiligen Sachverhalte als Gründe und die Gegensachverhalte als Folgen gerichtet usw. ${ }^{172}$

Wie im Alltagsleben ist auch im wissenschaftlichen Denken das jeweilige Gegenstandsgebiet, das die Wissenschaft untersucht, das Endziel des Gerichtetseins des wissenschaftlichen Urteilens. Der Naturwissenschaftler urteilt nicht zum Gedankenspiel, sondern er urteilt, um die Natur als Gegenstand zu erkennen und bestimmen. Und die formale Logik kann auch innerhalb dieser Einstellung arbeiten sie ist dann in formaler Allgemeinheit auf alle Gegenstandsgebiete gerichtet:

Die Analytik als formale Wissenschaftslehre ist wie die Wisssenschaften selbst ontisch gerichtet, und zwar vermöge ihrer apriorischen Allgemeinheit ontologisch. Sie ist formale Ontologie. Ihre apriorischen Wahrheiten sagen aus, was für Gegenstände überhaupt, für Gegenstandsgebiete überhaupt in formaler Allgemeinheit gilt, in welchen Formen sie überhaupt sind bzw. nur sein können. (Hua XVII, 125)

Durch die Formalisierung lässt die formale Logik die sachhaltigen „Kerne“ der besonderen Gegenstandsgebiete in unbestimmter Allgemeinheit als Etwas-überhaupt, als Eigenschaft überhaupt usw. bleiben. Damit bekommt sie die bloßen Formen des Gegenstands als Gegenstandskategorien. In dieser Einstellung ist die formale Logik die formale Ontologie. ${ }^{173}$

\footnotetext{
${ }^{171}$ Ebd., S.137.

${ }^{172}$ Ebd., S.117. Dazu vgl. auch Hua XIX/1, S.108: „Vollziehen wir den Akt und leben wir gleichsam in ihm, so meinen wir natürlich seinen Gegenstand und nicht seine Bedeutung. Wenn wir z.B. eine Aussage machen, so urteilen wir über die betreffende Sache und nicht über die Bedeutung des Aussagesatzes, über das Urteil im logischen Sinne.“ ${ }^{173}$ Vgl. Robert Sokolowski, Husserlian Meditations. How Words present Things, S.277.
} 
Doch es ist immer möglich, dass das Urteil zum Thema des urteilenden Subjektes wird:

Jederzeit ist aber, wie selbstverständlich, eine Änderung der Einstellung möglich, in der wir unsere Urteile, ihre Bestandstücke, ihre Verbindungen und Beziehungen zum Thema machen; das geschieht in einem neuen Urteilen zweiter Stufe, in einem Urteilen über Urteile, in dem Urteile zu Gegenständen der Bestimmung werden. Ohne diese Einstellungsänderung könnten wir natürlich keinen Begriff von Urteil und von seinen Urteilssyntaxen bekommen. (a.a.O., 117)

Das heißt, dass man von der geradehin urteilenden Einstellung in eine neue Einstellung übergehen kann, in der nicht die Gegenstände schlechthin, sondern ihre entsprechenden Urteilsbedeutungen das Thema sind. Diese neue Einstellung nennt Husserl die kritische Einstellung.

\section{b) Die kritische Einstellung}

In normalen Fällen setzt das gerade Urteilen die Gegenständlichkeit in Seinsgeltung, das heißt, für den Urteilenden ist die Gegenständlichkeit, so wie sie im Urteilen vermeint ist, eben eine wirkliche Gegenständlichkeit. Wenn das Urteil durch eine entsprechende Erfahrung bewährt wird, dann sagt der Urteilende mit Recht: „Es ist wirklich so, wie ich meinte." Aber schon im alltäglichen Urteilen besteht die Möglichkeit, dass die Erfahrung, die den Gegenstand-worüber gibt, das Urteilen nicht bewährt, sondern enttäuscht. In diesem Fall wird der Urteilende sagen: „Der Sachverhalt besteht wirklich nicht, wie ich meinte." Dabei entsteht für das urteilende Subjekt die Differenzierung des vermeinten Gegenstandes als vermeinten und des wirklichen Gegenstandes:

Damit scheiden sich gelegentlich auch für den Urteilenden die vermeinten

Gegenständlichkeiten als vermeinte ${ }^{174}$, rein als die in seinen Urteilsaktionen in

\footnotetext{
${ }^{174}$ In einer Fußnote sagt Husserl, dass die vermeinte Gegenständlichkeit als solche eben das Noema des Urteilens ist und er beruft sich dabei auf den Begriff des Noema in Ideen I (Formale und transzendentale Logik, S.131). Dabei stellt sich die klassische Frage über Husserls Begriff des Noema, ob der Unterschied zwischen Gegenstand schlechthin und vermeintem Gegenstand als solchem ein ontologischer Unterschied ist. Darauf antworten
} 
der und der kategorialen Gestalt zur setzung gekommenen, rein als das im syntaktischen Gang des Setzens Gesetzte als solches, von den entsprechenden ,wahren“ oder „wirklichen“ Gegenständlichkeiten. (a.a.O.,

Sobald diese Differenzierung stattfindet, ist der Urteilende in der Lage, seinen Blick von dem wirklichen Gegenstand abzulenken und ihn auf den vermeinten Gegenstand als vermeinten zu werfen. Er kann sich fragen, „Ist das, was ich vermeinte, wahr?“, er kann urteilen, „Was ich vermeinte, ist falsch“ , „Was ich vermeinte, ist inkonsistent" usw. ${ }^{175}$ Diese Urteile sind nicht über den Gegenstand schlechthin, sondern über den vermeinten Gegenstand als vermeinten, sie sind Urteile (Propositionen) zweiter Stufe. Während das Urteilen erster Stufe in der geradehin urteilenden Einstellung stattfindet, ist das Urteilen zweiter Stufe nur in der kritischen Einstellung vollzogen:

Jederzeit ist aber, wie selbstverständlich, eine Änderung der Einstellung möglich, in der wir unsere Urteile, ihre Bestandstücke, ihre Verbindungen und Beziehungen zum Thema machen; das geschieht in einem neuen Urteilen zweiter Stufe, in einem Urteilen über Urteile, in dem Urteile zu Gegenständen der Bestimmung werden. Ohne diese Einstellungsänderung könnten wir natürlich keinen Begriff von Urteil und von seinen Urteilssyntaxen bekommen. (a.a.O., 117)

Die vermeinten Gegenstände als vermeinte bezeichnet Husserl manchmal auch als die Verimeinten als solche, Vermeintheiten, oder einfach Meinungen. Husserl zufolge sind Meinungen eben die Urteilsbedeutungen, die die Apophantik untersucht:

Mit diesem Vermeinten als solchem, dem bloßen Korrelat des „Meinens“ (sprachlich auch oft Meinung $\delta o ́ \xi \alpha ~ g e n a n n t)$ haben wir nun in den Griff bekommen, was in der traditionellen Logik Urteil (Apophansis) heißt

verschiedene Interpretationen des Noema verschieden. Nach der West-Coast-Interpretation ist das vermeinte als solches eines Urteilens über physische Entitäten (z.B. den Baum schlechthin) eine ideale Entität, als ideale Entität hat es gar keine physischen Eigenschaften. Wegen des ontologischen Unterschiedes kann man die beiden nicht identifizieren. Nach Drummonds Interpretation geht der Unterschied zwischen den beiden nur auf verschiedene Betrachtungsweisen des identischen Gegenstandes zurück. Mit diesen Interpretationen werde ich mich im zweiten Abschnitt der vorliegenden Arbeit (im [\$44]) auseinandersetzen.

${ }^{175}$ Vgl. Hua XVII: Formale und transzendentale Logik, S.128. 
und Thema der apophantischen Logik ist. (a.a.O., 131)

Statt Meinung können wir auch Sinn sagen und von den Aussagen her auch von deren Bedeutungen sprechen. Nach der Bedeutung oder dem Sinn einer Aussage fragen und ihn sich deutlich machen ist offenbar nichts anderes als von der geraden aussagend-urteilenden Einstellung, in der wir nur die betreffenden Gegenstände ,haben“, übergehen in die reflektierte, in die Einstellung, in der die entsprechenden Gegenstandsmeinungen, Sachverhaltsmeinungen zur Erfassung oder Setzung kommen. Somit können wir diese Region auch als die der Sinne bezeichnen. (a.a.O., 138)

In den Zitaten identifiziert Husserl das Vermeinte als solches mit der entsprechenden Bedeutung, die eben das Urteilsbedeutung erster Stufe ist. Die Urteile zweiter Stufe haben also die Urteile erster Stufe zum Gegenstand. Im Vergleich dazu sind Urteile erster Stufe Urteile, die die Gegenstände schlechthin thematisieren.

Beim alltäglichen Denken geht man nur gelegentlich in die kritische Einstellung über, denn es ist nicht immer so, dass man beim alltäglichen Urteilen versucht, die Vermeintheiten als solche, nämlich die gefällten Urteile zu bewähren. Aber es verhält sich ganz anders beim wissenschaftlichen Denken, denn für den Wissenschaftler ist die kritische Einstellung berufsmäßig erfordert. Der Wissenschaftler versucht, Urteile erster Stufe über das jeweilige Gegenstandsgebiet (Natur, Kultur, usw.) zu fällen. Anders als der alltäglich Denkende darf er aber keine Urteile erster Stufe fällen, als diejenigen, die „,durch Adäquation an die Sachen selbst ihre „Richtigkeit“, ihre „Wahrheit“" ausgewiesen haben“(a.a.O., 129-130, 134). Hier ist Richtigkeit und Wahrheit ein Prädikat der Urteile, der Wissenschaftler muss also sich momentan von dem Gegenstandsgebiet distanzieren und das fragliche Urteil kritisieren, und dafür muss er auf das Urteil reflektieren, d.h. in die kritische Einstellung übergehen:

Das Endergebnis der Kritik ist - ideal gesprochen - „Wahrheit“, bzw. „Falschheit““. Diese Wahrheit besagt richtiges, kritisch bewährtes Urteil, bewährt durch Adäquation an die entsprechenden kategorialen Gegenständlichkeiten ,selbst“ ... Aus dieser Adäquation, also aus dem erfüllenden Übergang zur Selbsthabe und aus der Reflexion auf die bloße 
Meinung und ihre Deckung mit der Meinung in der Fülle entspringt der Begriff jener Richtigkeit, die den einen, den kritischen Begriff von Wahrheit ausmacht, denjenigen, wonach das Urteil wahr ist. (a.a.O., 132)

Beim wissenschaftlichen Denken muss man also beständig - im Vergleich zu dem alltäglichen Denken, bei dem er nur gelegentlich die kritische Einstellung annimmt - in die kritische Einstellung übergehen, ${ }^{176}$ denn er darf nur die Urteile gelten lassen, die sich durch die Kritik der Meinungen als richtig herausstellen, sonst kann er sie praktisch höchstens als vernünftige Hypothesen annehmen.

Es ist zu bemerken, dass beim wissenschaftlichen Denken ,die Thematik der Urteilssätze nur eine vermittelnde ist“(a.a.O., 134), obwohl die Urteilskritik sich in der Wissenschaft nicht gelegentlich, sondern beständig vollziehen muss. Denn das Ziel der Wissenschaftler ist, den wirklichen Gegenstand zu erkennen, nämlich richtige Urteile erster Stufe über den wirklichen Gegenstand zu fällen. Man kann sagen, dass die Wissenschaften sich in einer Mischung der beiden Einstellungen bewegen, wobei die geradehin urteilende Einstellung immer die primäre Einstellung ist, während die kritische Einstellung nur eine sekundäre Rolle spielt. Das gilt auch für das alltägliche Denken, nur dass der Zickzack der Einstellungen beim alltäglichen Denken nicht beständig, sondern nur gelegentlich ist.

Die formale Logik kann auch von der geradehin urteilenden Einstellung, in der sie in formaler Allgemeinheit über alle Gegenstandsgebiete urteilt und somit als formale Ontologie fungiert, in die kritische Einstellung übergehen. In der kritischen Einstellung arbeitet sie auch in formaler Allgemeinheit, aber nicht über Gegenstände schlechthin, sondern über vermeinte Gegenstände als vermeinte, nämlich Urteilsbedeutungen. Durch Formalisierung der Urteilsbedeutungen bekommt sie die Bedeutungskategorien, die zum Thema der formalen Apophantik angehören. Wenn die formale Logik die geradehin urteilende Einstellung verlässt und sich ausschließlich in der kritischen Einstellung bewegt, dann fungiert sie als formale Apophantik.

\footnotetext{
${ }^{176}$ Ebd., S129-130. Dazu vgl. Robert Sokolowski, Husserlian Meditations. How Words present Things, S.279: „The scientist, says Husserl, lives in such a zigzag motion between supposition and facts; this awareness and motion defines him as a scientists.“
} 


\section{c) Zusammenfassung des Paragraphen}

Durch die obigen Ausführungen lässt sich die Zweigliederung der formalen Logik in Ontologie und Apophantik subjektiv begründen. Der Gegenstand schlechthin ist das Thema des Urteilenden, der in der geradehin urteilenden Einstellung lebt. Wenn er in dieser Einstellung formale Logik betreibt, dann beschäftigt er sich mit den Gegenstandsformen, d.i. den Gegenstandskategorien - er betreibt formale Ontologie. Im Vergleich dazu wird die Urteilsbedeutung zu seinem Thema, wenn er in die kritische Einstellung eintritt. Betreibt er in der kritischen Einstellung formale Logik, dann beschäftigt er sich mit den Bedeutungskategorien - er betreibt formale Apophantik. Die Zweigliederung der formalen Logik ist also auf die Doppeleinstellung zurückzuführen. Die Unterscheidung von Gegenstand/Bedeutung und die von Gegenstandskategorie/Bedutungskategorie sind auch durch die beiden Einstellungen, in denen sie jeweils zum Thema des Urteilenden werden, phänomenologisch erklärt.

Was lässt sich in kritischer Einstellung innerhalb der formalen Logik untersuchen? Die innere formale Struktur der Urteilsbedeutungen, die Husserl als das Eigenwesentliche $^{177}$ der Urteile bezeichnet. Man fragt, ob eine bestimmte Form eine einheitliche Urteilsbedeutung bilden kann (Fomenlehre der Bedeutungen), ob die Form konsistent oder inkonsistent ist (Konsequenzlogik der Bedeutungen), ohne sich darum zu kümmern, ob ein Urteil der fraglichen Form wirklich wahr sein kann oder nicht. In diesem Sinn bilden die Formenlehre und die Konsequenzlogik der Bedeutungen die apophantische Logik im prägnanten Sinn. ${ }^{178}$ Im Vergleich dazu überschreitet die Apophantik auf der Stufe der Wahrheitslogik das Eigenwesentliche der Urteile, da die Frage nach Wahrheit schon die Dimension der Gegenstandsseite einführt, in Bezug auf die die Urteile zur Adäquation zu bringen sind und somit das Prädikat Wahrheit oder Richtigkeit gewinnen. Apophantik als Wahrheitslogik nimmt also eine Mittelstellung ${ }^{179}$ im Übergang von formaler Apophantik zu formaler Ontologie ein.

\footnotetext{
${ }^{177}$ Hua XVII: Formale und transzendentale Logik, S.68, 145, 146

178 Ebd., S.142.

${ }^{179}$ Ebd., S.150-151.
} 


\section{§24. Das Erkenntnisinteresse}

Was die geradehin urteilende Einstellung von der kritischen Einstellung unterscheidet, ist, wie gezeigt, ob der Urteilende den Gegenstand schlechthin thematisiert. Im wissenschaftlichen Denken tritt man beständig in die kritische Einstellung ein und kritisiert die Urteile, aber die Kritik dient nur dazu, wahre Urteile erster Stufe über das jeweilige Gegenstandsgebiet zu fällen. Deshalb ist die geradehin urteilende Einstellung beim wissenschaftlichen Denken immer die primäre Einstellung, und die kritische Einstellung nur sekundäre. Anders gesagt, das Erkenntnisinteresse über das Gegenstandsgebiet geht durch das wissenschaftliche Denken hindurch.

Wer formale Ontologie betreibt, befindet sich auch primär in der geradehin urteilenden Einstellung und hat Erkenntnisinteresse für alle Gegenstandsgebiete angesichts der Formen. Für ihn sind Mengen, Anzahlen, Ordnungen, usw. keine bloß gedanklichen Produkte, sondern Formen, die auf alle Gegenstandsgebiete anwendbar sind. Ein Mathematiker, der Arithmetik, Mengenlehre oder andere formale mathematische Disziplinen betreibt, und zugleich die Forschungsergebnisse als auf alle wirklichen Gegenstandsgebiete anwendbar sieht, betreibt bewußt die Mathematik in einem ,spezifisch logischen Sinn“(Hua XVII, 144), d.i. die formale Ontologie.

Allerdings arbeiten manche Mathematiker nur im Rahmen der ,,reinen“ Mathematik. Sie beschäftigen sich mit mathematischen Objekten (Mengen, Anzahlen usw.) nur hinsichtlich ihrer ,,mathematischen Existenz ${ }^{\text {180 }}$ - ihre theoretische Sorge ist nur, ob die mathematischen Urteile über mathematische Objekte in einem deduktiven System miteinander konsistent sind. Für sie ist es gleichgültig, ob die deduktiv-mathematischen Systeme auf Gegenstandsgebiete anwendbar sind und damit ihren eigentlich logischen Sinn gewinnen, der sie zu formal-ontologischen Disziplinen macht. (a.a.O., 143-145) Einfach gesagt, sie haben kein Erkenntnisinteresse für die wirklichen Gegenstände. Husserl zufolge sind die theoretischen Themen der reinen Mathematiker eigentlich nicht die Formen der wirklichen Gegenstände, sondern

\footnotetext{
${ }^{180}$ Ebd., S.144: „So versteht es sich, daß es für eine (unbewußt wie bewußt) „reine“ formale Mathematik keine anderen Erkenntnissorgen geben kann als die der ,Widerspruchslosigkeit“, als die unmittelbarer oder mittelbarer analytischer Konsequenz und Inkonsequenz, wohin offenbar alle Fragen mathematischer „Existenz“" gehören.“
} 
Formen der entsprechenden Urteilsbedeutungen bzw. der vermeinten Gegenstände als vermeinter, die eben das Thema der formalen Apophantik sind. Mit Husserls eigenen Worten, bei solcher Reduktion auf reine Mathematik verläßt die formale Ontologie ihren „spezifisch logischen Sinn“ - wer reine Mathematik betreibt, betreibt tatsächlich nicht formale Ontologie, sondern formale Apophantik. Husserl schreibt:

Es muss gesehen werden, dass eine formale Mathematik im Sinne jener reduzierten Reinheit ihr Eigenrecht hat und dass für die Mathematik jedenfalls keine Notwendigkeit besteht, über diese Reinheit hinauszugehen. Zugleich aber ist es ein großer Fortschritt philosophischer Einsicht, dass diese einschränkende Reduktion der logischen Mathesis (der zu wensenmäßiger Vollständigkeit gebrachten formalen Logik) auf eine pure Analytik der Widerspruchslosigkeit ihren wesentlichen Sinn hat als eine Wissenschaft, die es mit nichts anderem als mit apophantischen Sinnen nach ihrem eigenwesentlichen Apriori zu tun hat, und dass damit endlich der eigentliche Sinn der ,formalen Mathematik“, der Mathematik, der alle eigentlich logische, d.i. wissenschaftstheoretische Intention fernbleibt - der Mathematik der Mathematiker - prinzipiell geklärt ist. Hier liegt die einzige rechtmäßige Unterscheidung zwischen formaler Logik und bloßer formaler Mathematik. (a.a.O., 146)

Nun ist es an der Zeit, den im [§22] erwähnten Widerspruch aufzulösen. Der (vermeintliche) Widerspruch lautet: Einerseits sagen wir, dass die formale Mathematik sich mit den Gegenstandsformen beschäftigt und somit als formale Ontologie gilt; andererseits ordnen wir sie als eine Abteilung der formalen Apophantik zu, die die Bedeutungsformen untersucht. Dieser Widerspruch ist nur ein scheinbarer Widerspruch, denn die Rede von Mathematik ist hier zweideutig. Die Mathematik im logischen Sinne (,,logische Mathesis“) ist eine formal-ontologische Wissenschaft, während die reine Mathematik (,die Mathematik der Mathematiker") als eine formal-apophantische Wissenschaft gilt. Beim Übergang vom ersteren zum letzteren findet bewußt oder unbewußt eine Themaverschiebung statt: eine Verschiebung von den wirklichen Gegenständen auf die vermeinten Gegenstände als solche bzw. die Urteilsbedeutungen. 
Ob ein Mathematiker formale Ontologie oder reine Mathematik betreibt, häng davon $\mathrm{ab}$, ob sein mathematisches Denken dem Erkenntnisinteresse für wirkliche Gegenstände dient.

Da die reine Mathematik nur die Konsistenz der Urteile berücksichtigt, ist sie eigentlich ein Teil der Konsequenzlogik. Aus diesem Grund vertritt Husserl die Meinung, dass die traditionelle Apophantik seit Aristoteles und die überlieferten reinformal-mathematischen Disziplinen zusammen eine einheitliche Wissenschaft bilden, die eben die Konsequenzlogik ist und von Husserl auch als pure Analytik bezeichnet wird. Die Wahrheitslogik nimmt, wie oben schon erwähnt, eine Mittelstellung zwischen purer Analytik und formaler Ontologie ein, da sie schon die Dimension der Gegenstandsseite einführt. Man kann sagen: die Wahrheitslogik hat zur Hälfte ein Erkenntnisinteresse für Gegenstandsgebiete. ${ }^{181} \mathrm{Im}[\S 20]$ haben wir die These: die Konsequenzlogik lässt sich in Wahrheitslogik umformulieren, da formale Urteilskonsistenz notwendige Bedingung für Urteilswahrheit ist. Eine ähnliche These gilt auch für reine Mathematik und formale Ontologie: die reine Mathematik lässt sich in formale Ontologie umformulieren, da formale Urteilskonsistenz die notwendige Bedingung dafür ist, dass die Gegenstände, die den Urteilsformen entsprechen, wirkliche Gegenstände sein können. ${ }^{182}$

\section{§25. Das Erkenntnisinteresse und die Formenlehre}

Der Import des (Halb)Erkenntnisinteresses führt die Konsequenzlogik in zwei Richtungen: in die apophantische Wahrheitslogik und die formale Ontologie. Nun stellt sich die Frage: kann das Erkenntnisinteresse auch die Formenlehre der Bedeutungen in eine Formenlehre führen, die sich nicht nur mit dem Eigenwesentlichen der Urteile beschäftigt, sondern auch sich darum kümmert, ob die grammatischen Formen der Bedeutungen auf den Gegenstandsgebieten ihre entsprechenden ontologischen Formen

\footnotetext{
${ }^{181}$ Hua XVII, S.150: ,Sowie wir ... die beiden Möglichkeiten, die widerspruchsloser gegenständlicher Sinne und die möglicher Gegenstände selbst in Beziehung setzen, also Fragen möglicher Richtigkeit aufwerfen, stehen wir in der eigentlichen Logik und alsbald nimmt in ihr die ganze Mathematik der Urteile als Sinne eine formal-ontologische Bedeutung an - und doch dürfen wir sie noch nicht ohne weiteres als formale Ontologie ansprechen.“

182 Ebd., S.149.
} 
haben?

Obwohl Husserl nicht davon gesprochen hat, glaube ich, dass der Formenlehre der Bedeutungen eine Formenlehre der Gegenstände entspricht, ganz wie die reine Mathematik die formale Ontologie als ihr Gegenstück hat. Nur grammatisch zulässige Urteile haben das Potential, entsprechende Gegenstände in der Wirklichkeit zu haben. Z.B. besteht in der Wirklichkeit gar kein Sachverhalt, der die Form „S ist und“ hat. In diesem Sinne sind auch die Gegenstände schlechthin grammatisch geregelt. Deshalb kann man nicht nur vom Grammatikalitätsphänomen der Bedeutungen, sondern auch vom Grammatikalitätsphänomen der Gegenstände sprechen. Die Rede vom Grammatikalitätsphänomen überträgt sich also von der Bedeutungssphäre auf die Gegenstandssphäre:

(Die Grammatikalitätsthese der Gegenstände)

Die Gegenstände der geradehin urteilenden Einstellung sind gewissen durch Bedeutungskategorien bestimmten grammatischen Gesetzen unterworfen. Das bezeichnen wir als das Grammatikalitätsphänomen (bezüglich) der Gegenstände.

\section{§26. Zusammenfassung und Ausblick in den nächsten Abschnitt}

Durch den Vergleich mit den empirischen Grammatiken (II.Kapitel) und anderen formal-logischen Disziplinen (III. Kapitel) haben wir erkannt, (1) dass die reine Grammatik die apriorischen Komplikationsgesetze der Bedeutungen untersucht, (2) dass sie eine Teildisziplin der umfassenden formalen Logik ist und (3) wie sie sich zu den empirischen Grammatiken und anderen formal-logischen Disziplinen im Rahmen der formalen Logik verhält. Damit haben wir einen relativ vollständigen Überblick über Husserls Idee der reinen Grammatik gewonnen.

Außerdem haben wir die Rede vom Grammatikalitätsphänomen bezüglich der Bedeutungen und der Gegenstände eingeführt. Wiederholen wir sie hier zur Erinnerung:

(Die Grammatikalitätsthese der Bedeutungen)
Ausdrucksbedeutungen sind gewissen durch Bedeutungskategorien 
bestimmten grammatischen Gesetzen unterworfen. Das bezeichnen wir als das Grammatikalitätsphänomen (bezüglich) der Bedeutungen.

(Die Grammatikalitätsthese der Gegenstände)

Die Gegenstände der geradehin urteilenden Einstellung sind gewissen durch Bedeutungskategorien bestimmten grammatischen Gesetzen unterworfen. Das bezeichnen wir als das Grammatikalitätsphänomen (bezüglich) der Gegenstände.

Bisher haben wir uns ausschließlich mit den Logischen Untersuchungen und dem ersten Abschnitt der Formalen und transzendentalen Logik beschäftigt. Da in diesen Texten die phänomenologische Reduktion im Sinne von Ideen I nicht systematisch vollzogen wird, ${ }^{183}$ sind wir bisher noch außerhalb der transzendentalen Phänomenologie geblieben. Im nächsten Abschnitt möchte ich in die Sphäre der transzendentalen Phänomenologie eintreten und betrachten, wie das Grammatikalitätsphänomen gemäß den in transzendentaler Phänomenologie durchgeführten noetisch-noematischen Analysen aussehen wird.

\footnotetext{
183 Die erste Auflage der Logischen Untersuchungen erschien vor Husserls Entdeckung der transzendentalphänomenologischen Methode. In der zweiten Auflage der Logischen Untersuchungen ist die transzendentale Phänomenologie nur vereinzelt, aber nicht gründlich entfaltet. Im I. Abschnitt der Formalen und transzendentalen Logik betreibt Husserl absichtlich keine transzendentale Phänomenologie, denn er will sie erst im II. Abschnitt einführen. Vgl. Dieter Lohmar, Edmund Husserls „,Formale und transzendentale Logik“, S.19.
} 


\title{
II. ABSCHNITT: \\ DAS GRAMMATIKALITÄTSPHÄNOMEN IM \\ RAHMEN DER TRANSZENDENTALEN \\ PHÄNOMENOLOGIE
}

\author{
I. KAPITEL \\ DIE PHÄNOMENOLOGISCHE REDUKTION
}

\section{§27. Der Cartesianische Weg}

Aufgabe des vorliegenden Abschnitts ist, wie gesagt, das Grammatikalitätsphänomen unter dem Gesichtspunkt der transzendentalen Phänomenologie zu betrachten. Dafür muss man zuerst in die Arbeitssphäre der transzendentalen Phänomenologie eintreten. Interpreten haben bei Husserl verschiedene Wege zur transzendentalen Phänomenologie unterschieden. ${ }^{184}$ Diese Wege, genauer gesagt, sind verschiedene Überlegungen, die uns motivieren, die

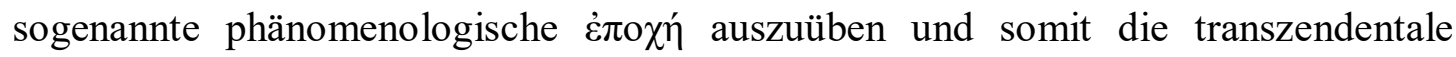
Phänomenologie zu betreiben. Hier begehen wir den sogenannten Cartesianischen Weg, denn die Textgrundlage, mit der wir uns hier beschäftigen, ist in großem Maße durch Cartesianische Überlegungen geprägt.

\section{§28. Kriterium der echten Wissenschaft}

Am Anfang der Cartesianischen Meditationen erinnert Husserl die Leser an Descartes Meditationes. Husserl zufolge ${ }^{185}$ versucht Descartes in den Meditationes die Philosophie als eine echte Wissenschaft ${ }^{186}$ zu etablieren. Dabei stellen sich drei Fragen:

\footnotetext{
${ }^{184} \mathrm{Vgl}$. Iso Kern, „Die drei Wege zur transzendental-phänomenologischen Reduktion in der Philosophie Edmund Husserls“, in: Tijdschrift voor Filosofie, 24st Jaarg., Nr.2(Juni 1962), Peeters Publishers/Tijdschrift voor Filosofie, S.303-349.

${ }^{185}$ Hua I: Cartesianische Meditationen und Pariser Vorträge, hrsg. von S. Strasser, Martinus Nijhoff, Den Haag 1950, S.48.

${ }^{186}$ Husserl unterscheidet auch zwischen exakten und deskriptiven Wissenschaften. Exakte Wissenschaften sind
} 
(1) Was ist das Kriterium für die Echtheit der Wissenschaft? (2) Hat irgendeine der historisch gewordenen Wissenschaften (Physik, Chemie usw.) schon das Echtheitskriterium erfüllt? (3) Gibt es eine mögliche Wissenschaft, die das Echtheitskriterium erfüllt, wenn die zweite Frage verneint wird? Diese Fragen nenne ich im Folgenden Kriteriumsfragen.

Einige von den faktisch oder historisch gewordenen Wissenschaften wurden als Vorbilder für andere angesehen. Z.B. zeigen die Mathematik und exakte Physik eine deduktive Methode, die andere Naturwissenschaften nachmachen. ${ }^{187}$ Es könnte der Gedanke naheliegen, dass das Echtheitskriterium sich durch vergleichende Untersuchung der vorbildlichen Wissenschaften herausarbeiten lässt - man vergleicht die vorbildlichen Wissenschaften, um ihren gemeinsamen Charakter als den der echten Wissenschaft zu erfassen. Nach diesem Gedanken würde eine Wissenschaft genau dann echt sein, wenn sie den Charakter der vorbildlichen Wissenschaften besitzt - z.B. wenn sie eine deduktiv-theoretische Gestalt wie die der Geometrie hat. Daraus ergibt sich ein Lösungsschema für die Kriteriumsfragen: (1) Die Echtheit einer Wissenschaft besteht darin, dass sie die Methoden der vorbildlichen Wissenschaften verwendet (wie induktive und deduktive Methode); (2) Selbstverständlich sind diejenigen historisch gewordenen Wissenschaften, die als Vorbilder gelten, echte Wissenschaften; (3) Die dritte Frage muss das Schema nicht beantworten, da die zweite Frage bejaht wird.

Eigentlich hat dieses Lösungsschema die Kriteriumsfragen nicht gelöst, sondern nur ein Stück verschoben. Es setzt einfach voraus, dass die vorbildlichen Wissenschaften echte Wissenschaften sind. Die Vorausetzung mag richtig sein, aber solange man sie nicht begründet hat, ist sie noch eine naive Voraussetzung. ${ }^{188}$ Aus

\footnotetext{
Mathematik, mathematische Physik usw., sie operieren mit den in deduktiven Systemen exakt bestimmten Begriffen wie „Punkt“”, „Linie““, „,Kraft“; deskriptive Wissenschaften sind z.B. Geschichte, Naturkunde usw., deren Begriffe nicht exakt bestimmt, sondern ,,morphologisch“ und etwas vage sind. (Ideen I, §§73ff.) Außerdem spricht Husserl auch von strenger Wissenschaft; nach David Bells Interpretation ist diese Strengheit eben die hier fragliche Echtheit der Wissenschaft. (Vgl. Edmund Husserl, „Philosophie als strenge Wissenschaft“, in Hua XXV, S.3.; David Bell 1990, S.158ff.)

187 „In der zeitgenössischen Philosophie, soweit sie Anspruch erhebt, ernste Wissenschaft zu sein, ist es fast zum Gemeinplatz geworden, daß es nur eine allen Wissenschaften und somit auch der Philosophie gemeinsame Erkenntnismethode geben könne. Diese Überzeugung entspricht vollkommen den großen Traditionen der Philosophie des 17. Jahrhunderts, die ja auch meinte, alles Heil der Philosophie hänge davon ab, daß sie die exakten Wissenschaften zum methodischen Vorbild nehme, vor allem also die Mathematik und die mathematische Naturwissenschaft." Hua II: Die Idee der Phänomenologie, hrsg. von Walter Biemel, Martinus Nijhoff, Den Haag 1950, S.23.

${ }^{188}$ Diesbezüglich wurde Descartes von Husserl kritisiert: „Descartes selbst hatte im voraus ein Wissenschaftsideal,
} 
diesem Grund bezeichne ich dieses Lösungsschema als das naive Lösungsschema für Kriteriumsfragen. Um diese Naivität $\mathrm{zu}$ überwinden, soll man zunächst das Echtheitskriterium artikulieren und dann fragen, ob und inwiefern die vorbildlichen Wissenschaften es erfüllen. Schließlich kommt man zurück zu Kriteriumsfragen.

Eine Alternative zu dem naiven Lösungschema geht auf Platon zurück. ${ }^{189}$ Platon stritt mit den sophistischen Skeptikern, die ,die prinzipelle Möglichkeit von so etwas wie „Philosophie“, wie Wissenschaft überhaupt“" ${ }^{190}$ leugneten; in Husserls Augen versuchte Platon, das platonische Ideal einer echten Wissenschaft herauszuarbeiten und deren Möglichkeit zu begründen. Für Platon bestimmt dieses Ideal den wesentlichen Charakter der echten Wissenshchaft, genau wie das Ideal des Staats betimmt, wie ein gerechter Staat aussehen soll. In diesem Sinne ist die Suche nach dem Ideal eben die nach dem Kriterium. War die Möglichkeit der Wissenschaft überhaupt im Streit mit der sophistischen Skepsis in Frage gestellt, so konnte Platon natürlich keine faktisch gewordene Wissenschaft als echte Wissenschaft naiv voraussetzen und von ihr das Ideal der echten Wissenschaft ablesen. ${ }^{191}$ Seine Wissenschaftslehre (Platons Dialektik) muss allen betriebenen Wissenschaften vorangehen und ihnen das Ideal oder das Echtheitskriterium im voraus zeigen. Hieraus ergibt sich ein platonisches Lösungsschema für Kriteriumsfragen, das die Richtung des naiven Lösungsschemas umkehrt: Man arbeitet zuerst das Echtheitskriterium heraus, ohne sich nach den faktisch-vorbildlichen Wissenschaften zu orientieren; erst danach überprüft er, ob die faktischen Wissenschaften, inklusive der vorbildlichen, das Echtheitskriterium erfüllen.

Husserl zufolge neigen die Wissenschaftslehren zu seiner Zeit dem naiven Schema zu. Er schreibt:

Aber diese [= Wissenschaftslehre - Anm.d.Verf.] selbst irrt in der neuesten Zeit von ihrem eigenen Sinn und ihrer unveräußerlichen Aufgabe ganz und gar ab.

\footnotetext{
das der Geometrie, bezw. der mathematischen Naturwissenschaft. Es bestimmt als ein verhängnisvolles Vorurteil die Jahrhunderte und bestimmt auch, kritisch unerwogen, die Meditationen selbst ... Das alles darf uns nicht bestimmen. Wir haben als Anfangende noch kein normatives Wissenschaftsideal in Geltung..." Hua I, S.48-49.

${ }^{189}$ Hua XVII: Formale und transzendentale Logik, S.5-7.

190 Ebd.

191 „So wurde Platon auf den Weg der reinen Idee geführt. Seine nicht den faktischen Wissenschaften abgelesene, sondern rein ideale, reine Normen gestaltende Dialektik, in unserer Rede seine Logik oder Wissenschaftslehre, hatte den Beruf, nun erst faktisch Wissenschaft möglich zu machen, sie praktisch zu leiten." Ebd.
} 
Statt die reinen Wesensnormen der Wissenschaft nach allen ihren Wesensgestaltungen $\mathrm{zu}$ verfolgen, um dadurch den Wissenschaften prinzipielle Leitung geben und ihnen Echtheit der Methodengestaltung und der Rechenschaftsabgabe in allen Schritten ermöglichen zu können, gefällt sie sich vielmehr darin, sich von den faktischen Wissenschaften, insbesondere den vielbewunderten Naturwissenschaften in ihrem Wissenschaftsideal und ihren Problemstellungen leiten zu lassen. ${ }^{192}$

Dem Zitat zufolge ist eine Wissenschaftslehre, die dem naiven Schema folgt, keine richtige Wissenschaftslehre; in Husserls eigenen Worten, sie „,irrt von ihrem eigenen Sinn und ihrer unveräußerlichen Aufgabe ganz und gar ab“. Er sieht sogar darin eine „Tragik der modernen wissenschaftlichen Kultur“. Und er plädiert für eine Wissenschaftslehre, die dem platonischen Schema (wie bei Platon und Decartes) folgt.

Bisher haben wir das platonische Lösungsschema nur negativ bestimmt, und zwar als Gegenrichtung des naiven Lösungsschemas. Verschiedene Philosophen könnten das platonische Schema auf verschiedene Weisen durchführen, hier interessiert uns nur Husserls Verfahrensweise. Um den Kriteriumsfragen eine platonische Lösung anzubieten, schlägt Husserl vor, dass wir uns zuerst in das „,Streben und Handeln““193 der Wissenschaftler einleben. Dieser Vorgehensweise des Einlebens wollen wir uns im folgenden Paragraphen zuwenden.

\section{§29. Wissenschaftliches Denken und Evidenz}

Man möchte fragen: Wenn die Echtheit der faktischen Wissenschaften in Frage gestellt ist, ist es dann noch sinnvoll, uns in das „Streben und Handeln“ der Wissenschaftler einzuleben, um das Echtheitskriterium herauszuarbeiten? Verfallen wir dann nicht zurück in das naive Lösungsschema? Nach Husserl sollte uns dieses Bedenken nicht verhindern.

Husserl zufolge versucht ein Wissenschaftler beim wissenschaftlichen Denken eine Theorie, die das Forschungsgebiet getreu charakterisiert, zu etablieren und

\footnotetext{
192 Ebd.

${ }^{193}$ Hua I: Cartesianische Meditationen und Pariser Vorträge, S.50.
} 
entwickeln. Er mag daran scheitern und nur eine vermeintliche Wissenschaft erzeugen, aber der Zweck seiner Betätigung bleibt klar. Husserl:

Mag uns auch hinsichtlich der Geltung der faktischen Wissenschaften (die sie prätendieren), also der Echtheit ihrer Theorien, korrelativ der Tragfähigkeit ${ }^{194}$ ihrer theoretisierenden Methoden, jede Stellungnahme versagt sein, nichts steht doch im Weg, uns in ihr wissenschaftliches Streben und Handeln einzuleben und somit uns auch klar und deutlich zu machen, worauf es damit eigentlich hinauswill. Treten wir so in fortschreitender Vertiefung in die Intention wissenschaftlichen Strebens, so entfalten sich uns die für die allgemeine Zweckidee echter Wissenschaft konstitutiven Momente, und zunächst in einer ersten Differenzierung. (Hua I, 50)

Das Bedenken, dass man durch das Einleben in den Wissenschaftler in das naive Lösungsschema verfallen würde, können wir entkräften: Beim Einleben interessiert uns nicht der tatsächliche Erfolg oder Misserfolg des Wissenschaftlers, sondern sein Zweck; der Zweck lässt sich beim Einleben feststellen, auch wenn die wissenschaftliche Betätigung von dem Zweck abweicht und die dabei erzeugte Wissenschaft das Echtheitskriterium nicht erfüllt. Die Gefahr des Verfallens besteht nur dann, wenn wir uns beim Einleben nur nach den tatsächlich erzeugten, prätendierten Wissenschaften orientieren, um das Echtheitskriterium herauszuarbeiten, aber nicht dann, wenn wir uns bei Suche nach Echtheitskriterium vom Zweck der wissenschaftlichen Betätigung leiten lassen. Aus diesem Grund ist das Einleben in die wissenschaftliche Betätigung nicht auf das naive Schema festgelegt.

Inwiefern führt das Einleben zu einer platonischen Lösung für Kriteriumsfragen? Husserl zufolge kann man gewisse konstitutive Momente des Echtheitskriteriums durch die Zweck-Enthüllung der wissenschaftlichen Betätigung entdecken, denn der Zweck einer Handlung verweist mehr oder weniger auf das Kriterium, das das Produkt oder die Folge der Handlung erfüllen soll. In diesem Zusammenhang lässt sich das

\footnotetext{
${ }^{194}$ D.i. die Fähigkeit der Methoden, wissenschaftliche Echtheit zu erreichen.
} 
Echtheitskriterium durch die Formulierung der „Zweckidee echter

Wissenschaft“ (a.a.O.) zu Tage fördern.

Fangen wir an, uns in das wissenschaftliche Streben und Handeln einzuleben, um klar zu machen, worauf es hinauswill. Zuerst bemerkt Husserl, dass ein Wissenschaftler Urteile (Propositionen) über die Gegenstände seines Forschungsgebiets erzeugen möchte. Und genauer: „Der Wissenschaftler will nicht bloß urteilen, sondern seine Urteile begründen.“(a.a.O., 51) Husserl:

... so kommen wir alsbald bei der genaueren Auslegung des Sinnes einer Begründung, bezw. einer Erkenntnis auf die Idee der Evidenz. In der echten Begründung erweisen Urteile sich als richtig, als stimmend, d.h. sie ist die Übereinstimmung des Urteils mit dem Urteilsverhalt (Sache bezw. Sachverhalt) selbst. Genauer gesprochen: Urteilen ist ein Meinen und im allgemeinen ein bloßes Vermeinen, es sei das und das... Aber dem steht eventuell gegenüber ein ausgezeichnetes urteilendes Meinen, (urteilend das und das bewußt-haben). Es heißt Evidenz. Statt in der Weise des bloß sachfernen Meinens ist in der Evidenz die Sache als sie selbst, der Sachverhalt als er selbst gegenwärtig, der Urteilende also seiner selbst inne. (a.a.O.)

Die Begründung eines Urteils besteht also darin, dass das Urteil sich als richtig erweist - es stimmt mit dem Gegenstand (Sachverhalt) überein. Korrelativ ist der begründete Urteilsakt (das Urteilen) nicht ein bloßes Vermeinen des Gegenstandes, sondern er enthält auch das „Innesein“ oder „Erfahrung“ des Gegenstandes selbst. Im Zitat nennt Husserl den begründeten Urteilsakt Evidenz ${ }^{195}$, denn ,Evidenz ist in einem

\footnotetext{
${ }^{195}$ Diese Rede (evidentes Urteilen = Evidenz) soll man vorsichtig aufnehmen. Husserl bezeichnet Erfahrung (z.B. Wahrnehmung) auch als Evidenz, als ob ein evidentes Urteilen, das aufgrund einer Wahrnehmung vollzogen wird, eben mit dieser Wahrnehmung identisch wäre. Aber ein evidentes Urteilen ist, wie Günther Patzig bemerkt, etwas Komplexes. „Es setzt sich zusammen erstens aus dem Vollzug eines Urteils, zweitens aus der Wahrnehmung eines Sachverhalts (real oder ideal), drittens aus der Einsicht, dass die im Urteil ausgesprochene Meinung dem wahrgenommenen Sachverhalt entspricht." Ein evidentes Urteilen muss also eine Bekanntschaft mit der Übereinstimmung zwischen Gemeintem und Gegebenem enthalten, anders gewendet: „,Diese Übereinstimmung wird in der Evidenz erlebt". Man möchte Husserl fragen, ob ein solcher Evidenzbegriff, der eine Bekanntheit zweiter Stufe, d.i. die Übereinstimmungsbekanntheit involviert, zu anspruchsvoll ist. Für die Beantwortung dieser Frage könnte Husserls Unterscheidung zwischen der schwächeren Form des Übereinstimmungsbewußtseins, in der das Subjekt die Übereinstimmung ,,vorhanden“ hat, aber nicht thematisiert, und der stärkeren Form, in der das Subjekt sie thematisch zum Gegenstand hat, revelant sein. (Vgl. Günther Patzig 2011, S.70; VI. LU, §§8, 39.)
} 
allerweitesten Sinne eine Erfahrung von Seiendem und So-Seiendem, eben ein Esselbst-geistig-zu-Gesicht-bekommen.“(a.a.O., 52)

Mit den Begriffen von Begründung und Evidenz glaubt Husserl, die Zweckidee (oder „das normative Wissenschaftsideal“ ${ }^{\text {“196) }}$ ) der Wissenschaft enthüllt zu haben. ${ }^{197}$ Provisorisch können wir sie wie folgt formulieren:

(Die Zweckidee echter Wissenschaft I)

Der Wissenschaftler soll beim wissenschaftlichen Streben und Handeln evidente Urteilsakte vollziehen und dabei evidente Urteile (Propositionen) erzeugen.

Husserl glaubt, wie Descartes, dass der Philosoph, der eine Wissenschaft (Philosophie) durch absolute Begründung etablieren will, mit evidenten Urteilen anfangen muss. ${ }^{198}$

\section{§30. Evidenz und Wissenschaft}

Für Husserl und den von ihm interpretierten Descartes genügt (die Zweckidee echter Wissenschaft I) noch nicht, die Anforderung, die eine echte Wissenschaft erfüllen muss, zu charakterisieren. Um dies zu erklären, wollen wir uns mit Husserls Evidenzbegriff näher auseinandersetzen.

\section{a) Apodiktische Evidenz}

Schon im Alltagsleben vollzieht man Urteilsakte, die die Anforderung (der Zweckidee echter Wissenschaft I) erfüllt. Wenn ich einen grünen Baum sehe und urteile, dass der Baum grün ist, dann vollziehe ich schon einen evidenten Urteilsakt. Denn der Urteilsakt vollzieht sich aufgrund einer Wahrnehmung, die Evidenz ist - sie ist Selbsterfassung des Urteilsgegenstandes in dem Modus ,es selbst“199. Allerdings ist es möglich, dass meine weiteren Erfahrungen es herausstellen, dass der Baum eigentlich

\footnotetext{
${ }^{196}$ Hua I: Cartesianische Meditationen und Pariser Vorträge, S.49.

${ }^{197}$ Ebd., S.6: „Ihrem Absehen nach soll nichts als wirklich wissenschaftlich gelten, was nicht durch vollkommene Evidenz begründet ist, d.h. auszuweisen ist durch Rückgang auf die Sachen oder Sachverhalte selbst in ursprünglicher Erfahrung und Einsicht.“

${ }^{198}$ Ebd.: „Davon geleitet machen wir anfangende Philosophen uns zum Prinzip, nur in Evidenz zu urteilen und die Evidenz selbst kritisch nachzuprüfen, auch das selbstverständlich wieder in der Evidenz.“

${ }^{199}$ Ebd., S.56: ,Jede Evidenz ist Selbsterfassung eines Seienden oder Soseienden in dem Modus „es selbst“ in völliger Gewißheit dieses Seins, die also jeden Zweifel ausschließt.“
} 
eine Mirage ist. Deswegen ist der Evidenzcharakter der Wahrnehmung und des darauf vollzogenen Urteilens immer der offenen Möglichkeit des Nichtseins oder Nichtsoseins ausgesetzt. ${ }^{200}$ Der Evidenzcharakter der Wahrnehmung und des darauf vollzogenen Urteilens ist also unvollkommen, in dem Sinn, dass sie die Möglichkeit des Nichtseins nicht ausschließt und nicht von jedem Zweifel befreit wird. Wegen ihrer Unvollkommenheit ist solche Evidenz nicht in der Lage, eine echte Wissenschaft vollständig zu rechtfertigen.

Im Vergleich dazu hat eine vollkommene Evidenz, so konzipiert Husserl, die ausgezeichnete Eigenheit, „dass sie nicht bloß überhaupt Seinsgewißheit der in ihr evidenten Sachen oder Sachverhalte ist, sondern sich durch eine kritische Reflexion zugleich als schlechthinnige Unausdenkbarkeit des Nichtseins derselben enthüllt; daß sie also im voraus jeden vorstellbaren Zweifel als gegenstandslos ausschließt. “201 Eine solche Evidenz, deren Gegenteil nicht ausdenkbar ist, nennt Husserl apodiktische Evidenz. ${ }^{202}$ Offensichtlich sind die äußere Wahrnehmung und das darauf vollzogene Urteilen keine apdiktische Evidenz.

Mit dem Begriff der apodiktischen Evidenz können wir die Zweckidee (oder „das normative Wissenschaftsideal") echter Wissenschaft genauer und anspruchsvoller formulieren:

(Die Zweckidee echter Wissenschaft II)

Der Wissenschaftler soll beim wissenschaftlichen Streben und Handeln nach Möglichkeit 203 apodiktisch-evidente Urteilsakte vollziehen und dabei apodiktisch-evidente Urteile (Propositionen) erzeugen.

Der Philosoph, der eine Wissenschaft (Philosophie) durch absolute Begründung etablieren will, muss also mit apodiktisch-evidenten Urteilen anfangen. ${ }^{204}$ Dabei wird

\footnotetext{
${ }^{200}$ Hua I: Cartesianische Meditationen und Pariser Vorträge, S.56.

${ }^{201}$ Ebd.

${ }^{202}$ Der Vollständigkeit haber ist zu ergänzen, dass Husserl in Cartesianischen Meditationen zwischen adäquater und apodiktischer Evidenz unterschieden hat. Beide sind für ihn vollkomme Evidenz. Bei einer adäquaten Evidenz handelt es sich um eine solche Evidenz, in der der Gegenstand vollständig und allseitig im Modus „es selbst" gegeben wird. Vgl. Ebd., S.55.

${ }^{203}$ Es ist also eine kantische Idee, der man sich immer nähern soll.

${ }^{204}$ Hua I, S.52: „Vollkommene Evidenz und ihr Korrelat, reine und echte Wahrheit, ist gegeben als eine dem Streben nach Erkenntnis, nach Erfüllung der meinenden Intention innewohnende, bezw. durch Einleben in solches Streben zu entnehmende Idee.“
} 
auch das Kriterium echter Wissenschaft geoffenbart und somit kann man die erste der Kriteriumsfragen beantworten: Eine echte Wissenschaft muss nach Möglichkeit auf der Basis von apodiktischer Evidenz aufgebaut werden.

\section{b) Die Evidenz für das Dasein der Welt}

Die Evidenz einer äußeren Wahrnehmung und eines Wahnehmungsurteils (qua Urteilsaktes) ist, wie gesagt, nicht apodiktisch. Im alltäglichen und wissenschaftlichen Denken verwirft man oft das Dasein von einzelnen in der Erfahrung gegebenen Gegenständen. Doch man glaubt immer, dass es die Welt gibt, auch wenn ihre einzelnen Mitgelieder manchmal zweifelhaft sind. Mit Husserls eigenem Wort, man will immer in der natürlichen Einstellung ${ }^{205}$ bleiben. Husserl beschreibt die natürliche Einstellung wie folgt:

Ich finde beständig vorhanden als mein Gegenüber die einen räumlichzeitliche Wirklichkeit, der ich selber zugehöre, wie alle anderen in ihr vorfindlichen und auf sie in gleicher Weise bezogenen Menschen. Die ,Wirklichkeit“, das sagt schon das Wort, finde ich als waches Ich in nie abbrechender zusammenhängender Erfahrung als daseiende vor und nehme sie, wie sie sich mir gibt, auch als daseiende hin. Alle Bezweiflung und Verwerfung von Gegebenheiten der natürlichen Welt ändert nichts an der Generalthesis der natürlichen Einstellung. „Die“ Welt ist als Wirklichkeit immer da, sie ist höchstens hier oder dort ,anders“ als ich vermeinte, das oder jenes ist aus ihr unter den Titeln „Schein“, „Halluzination“ u. dgl. sozusagen

\footnotetext{
${ }^{205}$ Man sollte die natürliche Einstellung von der sogenannten naturalistischen Einstellung, von der Husserl auch gelegentlich spricht, unterscheiden. Husserl verbindet mit dem Terminus ,,naturalistisch“ zwei verschiedene Sinne: (1) Die naturalistische Einstellung ist die Tendenz, alles, was ist, auf die bloß physische Naturwelt, die die Physik mathematisch behandelt, zu reduzieren. Ein natürlich Eingestellter muss solche naturalistische Tendenz nicht haben - z.B. kann ein Laie von Naturwissenschaften sich auf die Existenz seiner Lebenswelt verlassen, ohne zu versuchen, sie naturalistisch zu interpretieren. Außerdem kritisiert Husserl die naturalistische Einstellung in diesem negativ konnotierten Sinne - dazu vgl. Krisis in Hua VI und Philosophie als strenge Wissenschaft in Hua XXV. (2) In Ideen II ist aber „naturalistische Einsetellung“ nicht in negativer Konnotation gemeint. Dabei meint Husserl eher eine Einstellung, in der das Subjekt den Gegenstand, der ihn interessiert, hinsichtlich der physischen Eigenschaften betrachtet, ohne die Tendenz, alles auf Physisches zu reduzieren. Das ist eine typische Einstellung von Naturwissenschaftlern. Dazu vgl. Hua IV: Ideen II, S.183.
} 
herauszustreichen, aus ihr, die - im Sinne der Generalthesis - immer daseiende

Welt ist. ${ }^{206}$

Die Generalthesis der natürlichen Einstellung, oder kurz, die natürliche Thesis, ist, dass es eine wirkliche Welt gibt. Husserl zufolge sind die historisch gewordenen Wissenschaften ausschließlich innerhalb der natürlichen Einstellung betrieben. ${ }^{207} \mathrm{Sie}$ alle setzen die natürliche Generalthesis, explizit oder implizit, voraus. ${ }^{208}$

Die Evidenz für diese Generalthesis sind nicht einzelne Wahrnehmungen oder Urteilsakte, sondern das beständige Erfahrungsleben, in dem ein und dieselbe Welt mir thematisch oder unthematisch gegenübersteht. Husserl fragt, ist diese Evidenz eine apodiktische Evidenz? ${ }^{209}$ Und er antwortet:

Was das eine anbelangt, so ist die universale sinnliche Erfahrung, in deren Evidenz uns die Welt beständig vorgegeben ist, offenbar nicht so ohne weiteres als eine apodiktische Evidenz in Anspruch zu nehmen, die also die Möglichkeit eines Zweifelhaftwerdens, ob die Welt wirklich sei, bzw. die ihres Nichtseins absolut ausschließen würde. Nicht nur daß Einzelerfahrenes die Entwertung als Sinnenschein erleiden kann, auch der jeweils ganze, einheitlich überschaubare Erfahrungszusammenhang kann sich als Schein erweisen, unter dem Titel zusammenhängender Traum. ${ }^{210}$

Es ist unklar, ob der Hinweis auf solche Umschläge des Erfahrungszusammenhangs wirklich die Denkmöglichkeit des Nichtseins der Welt beweisen kann. ${ }^{211}$ Husserl will nicht ohne weiteres behaupten, dass die Evidenz für das Dasein der Welt nicht apodiktisch ist. Aber da es auch nicht sicher ist, ob sie apodiktisch ist, schlägt Husserl

\footnotetext{
${ }^{206}$ Hua III/1: Ideen zu einer reinen Phänomenologie und phänomenologischen Philosophie. Erstes Buch. Allgemeine Einführung in die reine Phänomenologie, hrsg. von Karl Schuhmann, Martinus Nijhoff, Den Haag 1976, S.61. 207 Ebd.

${ }^{208}$ Hua I, S.57: „Auf die Welt bezieht sich das alltäglich handelnde Leben, auf sie auch beziehen sich alle Wissenschaften, unmittelbar die Tatsachenwissenschaften, mittelbar als Instrumente der Methode die apriorischen. Allem voran ist das Sein der Welt selbstverständlich - so sehr, daß niemand daran denken wird, es ausdrücklich in einem Satz auszusprechen.“

${ }^{209}$ Ebd.: „Haben wir doch die kontinuierliche Erfahrung, in der uns diese Welt immerfort als fraglos seiende vor Augen steht. Aber so sehr diese Evidenz an sich früher ist als alle Evidenzen des weltzugewandten Lebens und aller Weltwissenschaften ... so werden wir doch bald bedenklich, wiefern sie in dieser Funktion apodiktischen Charakter beanspruchen kann.“”

${ }^{210}$ Ebd.

${ }^{211}$ Das, dass sich der ganze bisherige Erfahrungszusammenhang in Bezug auf die Welt als ,zusammenhängender Traum" erweist, heißt nicht unbedingt, dass die Welt nicht existiet, es kann sein, dass die Welt wirklich existiert, obwohl sie nicht so ist, wie ich sie bisher erfahren habe.
} 
vor, zu vermeiden, zum Zweck der Begründung einer echten Wissenschaft auf die Evidenz für das Dasein der Welt zurückzugreifen. ${ }^{212}$

Die zweite Kriteriumsfrage, ob irgendeine der historisch gewordenen Wissenschaften (Physik, Mathematik, Chemie usw.) schon das Echtheitskriterium erfüllt, können wir jetzt beantworten. Die Antwort lautet „Nein“, und zwar aus zwei Gründen: (1) Für sie (besonders die Naturwissenschaften wie Physik, Chemie usw.) sind die Forschungsgegenstände durch Erfahrung gegeben und dann naturalistisch ${ }^{213}$ interpretiert, während die Erfahrung, wie gesagt, keine apodiktische Evidenz liefert. (2) Sie alle sind betrieben innerhalb der natürlichen Einstellung und setzen somit das Dasein der Welt voraus. Und es ist fraglich, ob die Evidenz für diese Voraussetzung apodiktisch ist.

Ein Philosoph, der eine Wissenschaft (Philosophie) durch apodiktische Evidenz etablieren will, darf also weder die historisch gewordenen Wissenschaften noch ihre Generalthesis verwerten - er muss sie alle, wie Husserl sagt, einklammern (oder ,,außer

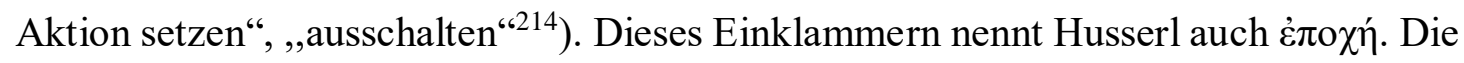

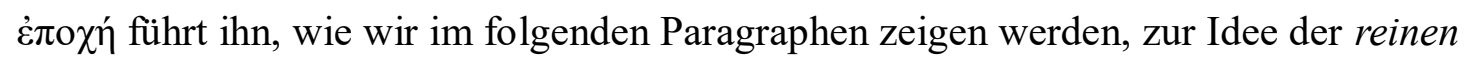
Phänomenologie.

Kritische Anmerkung: David Bell kritisiert, dass die durch Cartesianische Überlegungen motivierte Phänomenologie die Intersubjektivität und Lebenswelt verpasst und eine Art Solipsismus begeht; Bells Meinung nach wird Husserl in seinen Spätwerken, die die Phänomenolgie durch andere Überlegungen motivieren, „den Taum“ einer apodiktischen Wissenschaft allmählich aufgeben und stattdessen die Intersubjektivität und Lebenswelt als Datum akzeptieren; und die neue Dimension von Intersubjektivität und Lebenswelt bringt reichere Ressourcen (z.B. die kulturellen und historischen Faktoren) für phänomenologische Analysen. Man möchte einwenden, dass

\footnotetext{
${ }^{212}$ Hua I, S.57-58: „Den Hinweis auf diese möglichen und vorkommenden Umschläge der Evidenz brauchen wir nicht schon als eine hinreichende Kritik der Evidenz in Anspruch zu nehmen und darin einen vollen Beweis für die Denkmöglichkeit eines Nichtseins der Welt trotz ihres beständigen Erfahrenseins zu sehen. Wir behalten nur so viel, daß die Evidenz der Welterfahrung zu Zwecken einer radikalen Wissenschaftsbegründung jedenfalls erst einer Kritik ihrer Gültigkeit und Tragweite bedürfte, daß wir sie also nicht fraglos als unmittelbar apodiktisch in Anspruch nehmen dürfen."

${ }^{213}$ Vgl. die Fußnote am Anfang des vorliegenden Paragraphen [\$31b].

${ }^{214}$ Hua III/1: Ideen I, S.63.
} 
Husserl in Krisis und $C M$ das Konstitutionsproblem der Intersubjektivität und Lebenswelt schon diskutiert. Grob gesagt entdeckt Husserl einerseits die Lebenswelt als das „Sinnesfundament“ der Mathematik und mathematischen Naturwissenschaften ${ }^{215}$, dann versucht er, zu analysieren, wie die transzendentale Subjektivität, die andererseits durch Cartesianische Überlegungen entdeckt ist, die Lebenswelt und andere Subjekte konstituiert. Allerdings könnte Bell erwidern, dass die Intersubjektivität durch Tradition, gemeinsame Praxis, Institution, Kultur, Geschichte usw. auch umgekehrt die individuelle Subjektivität konstituiert. Nicht nur die Subjektivität konstituiert die Intersubjektivität, sondern auch umgekehrt. Husserls durch Cartesianische Überlegungen motivierte Phänomenologie scheint diese andere Konstitutionsrichtung vernachlässigt zu haben, auch wenn das Thema von Lebenswelt und Intersubjektivität schon offiziell eingeführt wird. Husserl beschreibt nur, wie andere Subjekte und Lebenswelt in der transzendentalen Subjektivität gegeben werden, als ob das Ego vom Himmel käme. Aber die Konstitution der anderen und Lebenswelt passiert nur gegen einen Hintergrund: das konstituierende Subjekt selber lebt in dieser Lebenswelt gemeinsam mit den anderen, kommt aus dieser Welt, und ist in dieser Welt erwachsen! In diesem Sinne halte ich Bells Kritik für zwingend. ${ }^{216}$

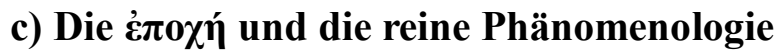

Erinnern wir uns an die dritte Kriteriumsfrage: Gibt es eine mögliche Wissenschaft, die das Echtheitskriterium erfüllt? Eine solche Wissenschaft muss auf apodiktischer Evidenz aufbauen. Nun sind die vorhandenen Wissenschaften und das Dasein der Welt schon eingeklammert, d.h., zur Begründung einer echten Wissenschaft (Philosophie) dürfen wir davon keinen Gebrauch machen. Aber was bleibt übrig, so fragt Husserl217, wenn die ganze Welt ausgeschaltet ist?

\footnotetext{
${ }^{215}$ In der Krisis kritisiert Husserl zurecht, dass Wissenschaftspraxis unter dem ideal-axiomatischen Standpunkt zu einer Vergessenheit der Lebenswelt geführt hat: man hält das Resultat des Standpunktes für die einzig reale Welt, missachtet und sogar ignoriert die Lebenswelt, auf die alle Wissenschaften zurückzuführen sind. Ich möchte ergänzen, dass nicht jede Wissenschaftspraxis einen universalen ideal-axiomatischen Standpunkt übernimmt, z.B. betreiben Kinder Mini-Mathematik, wenn sie arithmetische Probleme lösen, ohne die Lebenswelt zu verlassen. ${ }^{216}$ Vgl. David Bell, Husserl, S.204.

217 „Was kann denn übrig bleiben, wenn die ganze Welt, eingerechnet uns Menschen, ausgeschaltet ist? Ist das Weltall nicht All des Seienden überhaupt? ... was kann als Sein noch setzbar sein, wenn das Weltall, das All der
} 
Die Wahrnehmung könnte veridisch oder nichtveridisch sein. Aber in Bezug auf gewisse ihrer Komponenten ${ }^{218}$, die man unter Erster-Person-Perspektive beschreiben kann, ist die Wahrnehmung, relativ zu dem Wahrnehmungsgegenstand, unzweifelhaft, gleichgültig, ob die Wahrnehmung veridisch ist oder nicht. Dasselbe gilt allgemein für intentionale Erlebnisse, die bei Descartes unter dem Titel cogitatio zusammengefasst werden. ${ }^{219}$ Außerdem schließen sich diese intentionalen Erlebnisse zu einem Bewußtseinsleben oder Bewußtseinsstrom oder (transzendentaler) Subjektivität

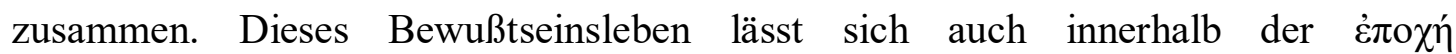

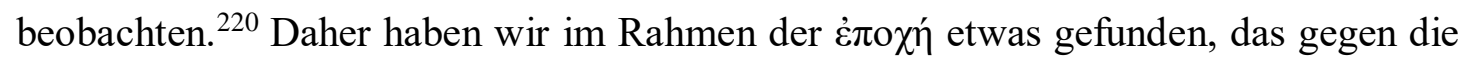
Existenz oder Nichtexistenz der weltlichen Gegenstände unempfindlich ist.

Die intentionalen Erblenisse und das Bewußtseinsleben sind relativ zu weltlichen

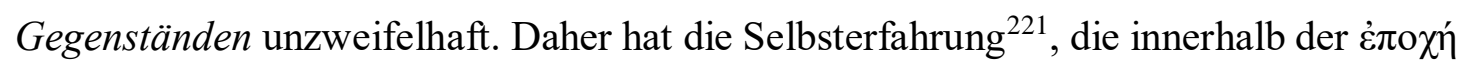
vollzogen wird, (relativ z.B. zu der dinglichen Wahrnehmung) eine bessere Chance, den Status der apodiktischen Evidenz zu gewinnen.

Hat Husserl dieser Selbsterfahrung den Status von apodiktischer Evidenz zugesprochen? Iso Kern zufolge hat Husserl seine Meinung geändert: In Ideen I und Fünf Vorlesungen beansprucht Husserl für sie ohne weiteres die apodiktische Evidenz, später (z.B. in Cartesianischen Meditationen) aber nicht. ${ }^{222}$ Schon die Vergangenheit des Bewußtseinslebens stellt die Frage, inwieweit die Erinnerung, durch die das vergangene Bewußtseinsleben zugänglich ist, apodiktisch sein kann? In

Realität eingeklammert bleibt?“" Hua III: Ideen zu einer reinen Phänomenologie und phänomenologischen Philosophie. Erstes Buch. Allgemeine Einführung in die reine Phänomenologie, hrsg. Walter Biemel, Martinus Nijhoff, Den Haag 1950, S.69-70. Für Fußnoten und Zitate von Ideen I verwende ich meistens die Version von Karl Schuhmann (1976) in Husserliana. Es wird besonders vermerkt, wenn ich mich auf Walter Biemels Version in Husserliana berufe.

${ }^{218}$ Smith und McIntyre unterscheiden zwischen den erlebnismäßigen (experiential) und extra-erlebnismäßigen (extra-experiential) Komponenten der Wahrnehmung. Die erlebnismäßigen Komponenten einer Wahrnehmung sind, grob gesagt, die Inhalte des Aktes, die man unter Erster-Person-Perspektive beschreiben kann. Im Vergleich dazu involvieren die extra-erlebnismäßigen Komponenten z.B. ein psychophysisches Verhältnis zwischen dem wahrnehmenden Menschen und dem wahrgenommenen Objekt, was die Erste-Person-Perspektive überschreitet. Vgl. David Woodruff Smith and Ronald McIntyre, Husserl and Intentionality, S.3-4.

${ }^{219}$ Hua II, S.30: „Und ebenso bei jeder Cogitatio. Wie immer ich wahrnehme, vorstelle, urteile, schließe, wie immer es dabei mit der Sicherheit oder Unsicherheit, der Gegenständlichkeit oder Gegenstandslosigkeit dieser Akte sich verhalten mag, im Hinblick auf das Wahrnehmen ist es absolut klar und gewiß, daß ich das und das wahrnehme, im Hinblick auf das Urteil, daß ich das und das urteile usw.“

${ }^{220}$ Vgl. Hua I: Cartesianische Meditationen und Pariser Vorträge, S.60-61.

${ }^{221}$ Auch transzendentale Erfahrung genannt. Ebd.

${ }^{222}$ Iso Kern, „Die drei Wege zur transzendental-phänomenologischen Reduktion in der Philosophie Edmund Husserls", S.310. 
Cartesianischen Meditationen will Husserl die Evidenz für das Bewußtseinsleben einer Kritik ihrer apodiktischen Tragweite unterziehen. ${ }^{223}$ Er fragt, „wie weit kann das transzendentale $\mathrm{Ich}^{224}$ sich über sich selbst täuschen und wie weit reichen die absolut zweifellosen Bestände trotz dieser möglichen Täuschung?“225 Tatsächlich wird diese methodische Kritik, wie Kern anmerkt, als „Problem höherer Stufe“ verschoben und nicht ausgeführt.

In Cartesianieschen Meditationen schlägt Husserl vor, dass wir uns zuerst der Selbsterfahrung widmen, und zwar ,unter Zurückstellung“ der Kritik über die Tragweite ihrer Apodiktizität. Denn diese Kritik ist vielmehr eine Aufgabe höherer

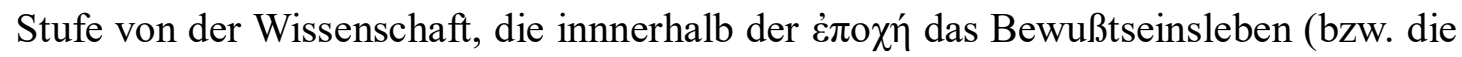
transzendentale Subjektivität) untersucht. Der Philosoph darf sich zuerst in einer ,,noch nicht im vollen Sinne philosophischen Stufe“ bewegen, d.h. zuerst in bloßer Hingabe an die Evidenz für das Bewußtseinsleben arbeiten und danach die höhere Aufgabe der Evidenzkritik thematisieren, ,ähnlich wie der Naturforscher in seiner Hingabe an die Evidenz der naturalen Erfahrung, wobei für ihn als Naturwissenschaftler Fragen einer prinzipiellen Erfahrungskritik überhaupt außerhalb seines Themas verbleiben“. ${ }^{226}$ Diese höhere Aufgabe wird, wie gesagt, ad calendas Graecas verschoben.

In diesem Kontext hat Husserl am Ende nicht festgestellt, ob die Evidenz des Bewußtseinslebens (bzw. der transzendentalen Subjektivität) apodiktisch ist. Husserl

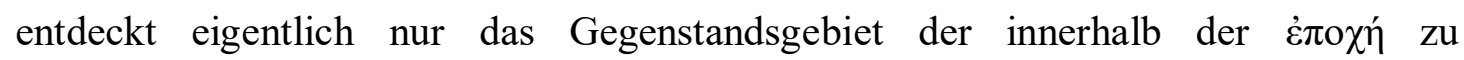
betrachtenden Subjektivität und somit eine Wissenschaft, die diese Subjektivität untersucht und als reine Phänomenologie zu bezeichnen ist. Die Phänomenologie hat, relativ zu den eingeklammerten Wissenschaften, eine bessere Chance, hinsichtlich der Existenz des Forschungsgegenstandes apodiktisch begründet zu werden; aber es bleibt in der Schwebe, ob das wirklich der Fall ist. Die dritte Kriteriumsfrage, ob es eine

\footnotetext{
${ }^{223}$ Hua I: Cartesianische Meditationen und Pariser Vorträge, $\S \S 9,13$.

${ }^{224}$ Hier mit dem Term „,transzendentales Ich“ ist, grob gesagt, das Subjekt des Bewußtseinslebens gemeint. Über technische Sinne von Husserls Begriffe des transzendentalen Ich vgl. David Bell, Husserl, S.204.

${ }^{225}$ Hua I: Cartesianische Meditationen und Pariser Vorträge, S.62.

${ }^{226}$ Ebd, S.68: „In der ersten [Stufe] wird das, wie sich alsbald zeigt, ungeheure Reich der transzendentalen Selbsterfahrung durchwandert werden müssen, und zunächst in bloßer Hingabe an die ihr im einstimmigen Verlauf innwohnende Evidenz, also unter Zurückstellung der Fragen einer letzten, auf apodiktische Prinzipien der Tragweite bedachten Kritik. Wir verfahren also in dieser noch nicht im vollen Sinne philosophischen Stufe ähnlich wie der Naturforscher in seiner Hingabe an die Evidenz der naturalen Erfahrung ...“
} 
Wissenschaft gibt, die auf der Basis von apodiktischer Evidenz aufgebaut wird, muss Husserl leider unbeantwortet lassen, denn sogar die reine Phänomenologie als der bisher konkurrenzfähigste Kandidat muss sich darum noch sehr anstrengen. ${ }^{227}$

Die dritte Kriteriumsfrage, ob es eine Wissenschaft gibt, die auf apodiktischer Evidenz (wenn auch nur hinsichtlich der Existenz des Forschungsgegenstandes) aufgebaut wird, dürfen wir außer Betracht lassen. Denn Ziel des vorliegen Kapitels ist nur zu zeigen, wie Husserl, unter Leitung der Cartesianischen Überlegungen, dazu

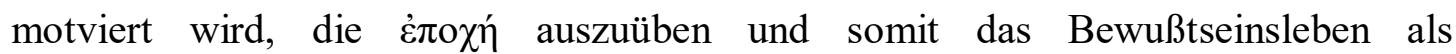
Forschungsfeld der reinen Phänomenologie festzustellen.

\section{§31. Zusammenfassung des Kapitels}

Dieses Ziel haben wir schon erfüllt: Descartes folgend will Husserl eine echte Wissenschaft begründen und er versucht, das Echtheitskriterium von Wissenschaften herauszuarbeiten ([§§28-29]); nach Husserl erfordert die echte Wissenschaft apodiktische Evidenz, aber die Evidenz für alle in natürlicher Einstellung betriebenen Wissenschaften ist nicht apodiktisch und sogar die Evidenz für das Dasein der Welt muss im Augenblick beiseitegeschoben werden ([§30a-b]); deshalb entscheidet er, die Methode der غ̇ंo $\chi \eta ́$ auszuüben - dann hat er gefunden, dass das Bewußtseinsleben (bzw.

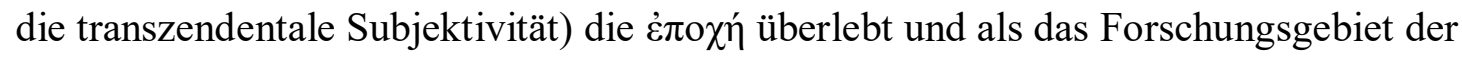
reinen Phänomenologie gelten soll ([§30c]).

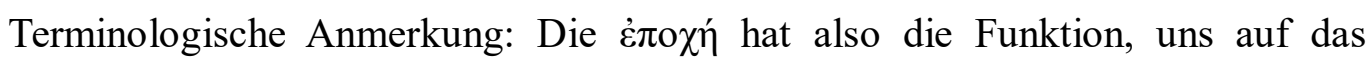
Bewußtseinleben, das die reine Phänomenologie untersucht, zurückzuleiten (reducere).

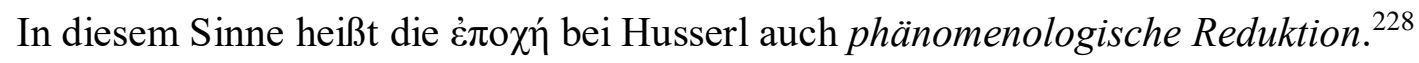

\footnotetext{
${ }^{227}$ Iso Kern ist der Meinung, dass schließlich nur zwei Momente des Bewußtseinslebens, nämlich die zeitliche Form und das Ich als ein universales Moment mannigfaltiger cogitationes, als apodiktische Momente übrig bleiben. Aber beide sind nur Formmomente (oder „nicht inhaltlicher Art“). Darüber vgl. Iso Kern, „Die drei Wege zur transzendental-phänomenologischen Reduktion in der Philosophie Edmund Husserls“, S.321.

${ }^{228}$ Hua I, S.61: ,,So geht ... dem natürlichen Sein der Welt ... voran als an sich früheres Sein das des reinen ego und seiner cogitationes. Der natürliche Seinsboden ist in seiner Seinsgeltung sekundär, er setzt beständig den transzendentalen voraus. Die phänomenologische Fundamentalmethode der transzendentalen غ̇ंoðท́, sofern sie auf ihn zurückleitet, heißt daher transzendental-phänomenologische Reduktion.“
} 


\section{KAPITEL}

\section{DIE NOETISCH-NOEMATISCHEN ANALYSEN IM RAHMEN DER TRANSZENDENTALEN PHÄNOMENOLOGIE I}

\section{§32. Intentionale und reelle Komponenten des intentionalen Erlebnisses}

\section{a) Intentionale und reelle Inhalte in den Logischen Untersuchungen}

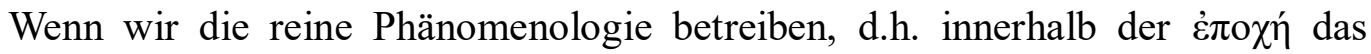
Bewußtseinsleben untersuchen, dann verlassen wir schon die natürliche Einstellung und treten in die phänomenologische. Die reine Phänomenologie fängt an mit der Unterscheidung zwischen intentionalen und reellen Komponenten (Inhalten) des intentionalen Erlebnisses. Allgemein gesprochen fungieren die reellen Komponenten eines Gegenstandes als dessen Bestandstücke und sie verhalten sich zu ihm wie Teile zum Ganzen. ${ }^{229}$ Z.B. sind Ziegel reelle Inhalte eines Hauses. Schon vor seiner Entwicklung der phänomenologischen Reduktion, und zwar in Logischen Untersuchungen, hat Husserl den Begriff des reellen Inhalts auf den Bereich der unter Erster-Person-Perspektive betrachteten intentionalen Erlebnisse angewandt. Bei solcher Anwendung versteht man unter reellem Inhalt eines intentionalen Erlebnisses den Gesamtinbegriff von allen unter Erster-Person-Perspektive vorfindlichen Inhalten, die als selbständige oder unselbständige Teile des intentionalen Erlebnisses und letztlich des Bewußtseinsstroms fungieren. ${ }^{230}$ Ebenso gehört auch ein ganzes intentionales Erlebnis zum reellen Bestand des Bewußtseinsstroms. In diesem Sinne sind Empfindungen ${ }^{231}$, Qualität und Materie als innere Momente des Aktes sowie deren Einheit (,das intentionale Wesen“ qua individuelles Moment) ${ }^{232}$ reelle Inhalte des intentionalen Erlebnisses.

\footnotetext{
${ }^{229}$ Hua XIX/1, S.231, 362-363. „Den Begriff Teil fassen wir in dem weitesten Sinne, der es gestattet, alles und jedes Teil zu nennen, was ,in“ einem Gegenstande unterscheidbar oder ... in ihm „vorhanden“ ist. Teil ist alles, was der Gegenstand im ,realen“, oder besser, reellen Sinne „hat", im Sinne eines ihn wirklich Aufbauenden.“

230 „Unter dem reellen phänomenologischen Inhalt eines Akes verstehen wir den Gesamtinbegriff seiner gleichgültig ob konkreten oder abstrakten Teile, mit anderen Worten, den Gesamtinbegriff der ihn reell aufbauenden Teilerlebnisse.“"Ebd., S.411, 413.

${ }^{231}$ Näheres darüber unten im [\$32c] der vorliegenden Arbeit.

${ }^{232} \mathrm{Vgl}$. [\$§4-5] der vorliegenden Arbeit.
} 
Wenn man in üblicher Rede von Inhalten eines Gegenstandes spricht, muss er nicht ausschließlich seine Bestandstücke meinen. In gewissem Sinne ist die Landschaft der Inhalt eines sie darstellenden Gemäldes, doch man will die Landschaft keineswegs als Bestandstück, nämlich reellen Inhalt des Gemäldes betrachten. Ähnliches gilt auch für intentionale Erlebnisse, denn die reellen Inhalte des Aktes erschöpfen nicht all seine Inhalte. ${ }^{233}$ Husserl zufolge enthält ein intentionales Erlebnis noch andersartige Inhalte, die er als intentionale Inhalte bezeichnet. In den Logischen Untersuchungen betrachtet Husserl (1) den intentionalen Gegenstand ${ }^{234}$, (2) die ideale Materie sowie (3) das intentionale Wesen in specie als intentionale Inhalte von intentionalen Erlebnissen. ${ }^{235}$

In Ideen I ist die Terminologie von reellen und intentionalen Inhalten auch nach der phänomenologischen Reduktion beibehalten, aber unter einer Revision. Einerseits differenziert Husserl die reellen Inhalte des intentionalen Erlebnisses weiter in hyletische und noetische. Audererseits ist es nicht klar, ob er die ideale Materie und das intentionale Wesen in specie noch für intentionale Inhalte halten will; mindenstens spricht er nur selten von diesen Spezies ${ }^{236}$ in seiner Diskussion über intentionale Inhalte. Stattdessen stehen die sogenannten noematischen Inhalte im Vordergrund als intentionale Inhalte. Wir wollen uns nunmehr diesem neuen Verständnis der beiden Inhaltsarten zuwenden.

\section{b) Das Noema als intentionaler Inhalt in Ideen I}

Zur Erörterung der in Ideen I revidierten Unterscheidung von reellen und intentionalen Inhalten ist es dienlich, wenn man sich, wie Husserl es in Ideen I oft tut, zuerst nach einer Wahrnehmungsanalyse orientiert und dann das Ergebnis verallgemeinert.

Angenommen, wir blicken auf einen grünen Baum im Garten. Wir üben die

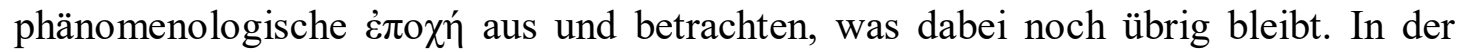

\footnotetext{
${ }^{233}$ Vgl. Ernst Tugendhat, Der Wahrheitsbegriff bei Husserl und Heidegger, Walter de Gruyter, Berlin 1970, S.33.

${ }^{234}$ Näheres darüber unten im [ $\left.\S 43 \mathrm{~b}\right]$ der vorliegenden Arbeit.

${ }^{235}$ Hua XIX/1: Logische Untersuchungen, S.413.

${ }^{236}$ Damit hängt zusammen, dass Husserl die Spezies-Auffassung von Bedeutungen schon vor Ideen I aufgegeben hat. Zu einer kurzen Diskussion darüber vgl. Wolfgang Künne, „Intentionalität: Bolzano und Husserl“, in: Versuche über Husserl, hrsg. von Stefania Centrone, Hamburg: Felix Meiner Verlag 2013, S.129-133.
} 
phänomenologischen Einstellung dürfen wir die intentionale Beziehung nicht als ein psychophysisches Verhältnis zwischen dem Menschen und dem Baum interpretieren, denn alle Naturwissenschften, inklusive der Physik und Psychologie, und sogar die Existenz des Menschen und des Baumes, sind in der phänomenologischen Einstellung eingeklammert. (Hua III/1, S.204) Doch die Wahrnehmung als cogitatio $^{237}$, unter Erster-Person-Perspektive betrachtet, ist noch übrig geblieben, und zwar mitsamt ihrem Sich-beziehen auf das cogitatum, ,den wahrgenommen Baum als solchen“(a.a.O.). Der Beisatz „als solcher“ deutet darauf hin, dass das cogitatum der fraglichen Baumwahrnehmung ausschließlich unter Erster-Person-Perspektive und in der phänomenologischen Reduktion beschrieben werden darf. Die gewünschte Beschreibung verhält sich neutral gegenüber natürlich eingestellten Feststellungen über die Existenz oder Nichtexistenz des Baumes. Denn solche Feststellungen sind im Rahmen der phänomenologischen Reduktion absichtlich ausgeschaltet. Husserl schreibt:

Und so fragen wir denn überhaupt, diese Ausschaltungen in ihrem klaren Sinn innehaltend, was in dem ganzen „reduzierten“ Phänomen evidenterweise ,liegt“. Nun dann liegt eben in der Wahrnehmung auch dies, daß sie ihren noematischen Sinn, ihr ,Wahrgenommenes als solches“ hat, „diesen blühenden Baum dort im Raume“ - mit den Anführungszeichen verstanden - eben das zum Wesen der phänomenologisch reduzierten Wahrnehmung gehörige Korrelat. (a.a.O., 209)

Mit den Termen „,noematischer Sinn“ (im laxen Sinne ${ }^{238}$ ), „Wahrgenommenes als solches“" und „, dieser blühende Baum in Anführungszeichen“ meint Husserl eben das, was er mit dem terminus technicus „Noema“ bezeichnet. Er glaubt, dass das Noema auch in der phänomenologisch reduzierten Wahrnehmung ,evidenterweise ,liegt“ “ “. Und er glaubt, dass man dieses Noema beschreiben kann. ${ }^{239}$

\footnotetext{
237 Über den Begriff von cogitatio vgl. [\$36] der vorliegenden Arbeit.

${ }^{238}$ Noematischer Sinn im strengen Sinne ist nur ein Moment des vollen Noema. Dazu vgl. [\$34] der vorliegenden Arbeit.

${ }^{239}$ Hua III/1, S.205:,,In unserer phänomenologischen Einstellung können und müssen wir die Wesensfrage stellen: was das „Wahrgenommene als solches“ sei, welche Wesensmomente es in sich selbst, als dieses WahrnehmungsNoema, berge. Wir erhalten die Antwort in reiner Hingabe an das wesensmäßig Gegebene, wir können das „Erscheinende als solches“ getreu, in vollkommener Evidenz beschreiben. Nur ein anderer Ausdruck dafür
} 
Ein Beispiel eignet sich, den Begriff des Noema deutlicher zu illustrieren. Mal angenommen, Sherlock Holmes hat einen Zauberpilz, der Halluzination auslösen kann, eingenommen und erleidet dann Halluzination von einem großen und grünen Baum in der Wohnung, die tatsächlich leer ist. Dr. Watson fragt Holmes, „Was sehen Sie?“ Der Detektiv, der sich nicht sicher ist, ob der Baum wirklich existiert, kann eine existenzneutrale und richtige Antwort geben: (i) ,Entweder sehe ich einen Baum, der grün, groß, usw. ist oder würde ich einen Baum, der grün, groß, usw. ist, sehen, wenn meine Wahrnehmung veridisch wäre.“ Im Vergleich dazu würde er eine existenzneutrale aber falsche Antwort geben, wenn er sagte: (ii) „Entweder sehe ich einen Baum, der rot, klein, usw. ist oder würde ich einen Baum, der rot, klein, usw. ist, sehen, wenn meine Wahrnehmung veridisch wäre. “240 Die Antwort (i), wenn innerhalb der phänomenologischen Reduktion interpretiert, ist schon eine phänomenologische Beschreibung des Wahrnehmungsnoema.

Husserl selber verwendet kein kontrafaktisches Konditional, um das Wahrnehmungsnoema zu beschreiben. Die Antwort (i) kann man mit Husserls eigenem Vokabular so übersetzen: (i’) Ich sehe ,einen grünen und großen Baum“ oder (i”)Ich sehe einen grünen und großen Baum als solchen. Die Anführungszeichen und der Beisatz ,,als solcher“ deuten darauf hin, das hier die Existenz oder Nichtexistenz des wahrgenommenen Gegenstandes für unsere noematische Beschreibung nicht in Frage kommt.

Nach Husserl ist das Wahrnehmungsnoema in der phänomenologisch reduzierten Wahrnehmung ein ,evident Gegebenes“ (Hua III/1, S.228), auch wenn der Wahrnehmungsgegenstand nicht wirklich existiert. Wenn man sich bei Betrachtung der Wahrnehmung ausschließliech an den reellen Komponenten orientiert, dann liegt die Ansicht nahe, dass das Noema ein Bestandstück, nämlich einen reellen Inhalt der Wahrnehmung ausmacht. ${ }^{241}$ Dagegen vertritt Husserl die Auffassung, dass das Noema obwohl der Wahrnehmung korrelativ zugehörig, ihrem reellen Inhalt jedoch

ist: ,die Wahrnehmung in noematischer Hinsicht beschreiben“. “

${ }^{240}$ Vgl. Christian Beyer, ,Fiktionale Rede“, in: Semantik und Ontologie. Beiträge zur philosophischen Forschung, hrsg. von Mark Siebel und Mark Textor, Ontos Verlag, Frank/Lancaster 2004, S.169-184.

${ }^{241}$ Ebd., S.207. Dazu vgl. auch [§11] der V. LU. 
transzendent ist (a.a.O.). Für ihn gehört das Noema zum intentionalen Inhalt der Wahrnehmung. Er schreibt:

Es gilt jetzt aber $\mathrm{zu}$ beschreiben, was davon als phänomenologisches Residuum verbleibt, wenn wir auf die „reine Immanenz“ reduzieren, und was dabei als reelles Bestandstück des reinen Erlebnisses gelten dürfe, und was nicht. Und da heißt es sich völlig klarmachen, daß zwar zum Wesen des Wahrnehmungserlebnisses in sich selbst der ,wahrgennommene Baum als solcher" gehört, bzw. das volle Noema, das durch die Ausschaltung der Wirklichkeit des Baumes selbst und der ganzen Welt nicht berührt wird; daß aber andererseits dieses Noema mit seinem „Baum“ in Anführungszeichen ebensowenig in der Wahrnehmung reell enthalten ist, wie der Baum der Wirklichkeit. (a.a.O., 226)

Auf den Unterschied und Zusammenhang zwischen den reellen Inhalten, dem Noema und dem wirklichen Gegenstand werden wir noch zu sprechen kommen. Im folgenden Paragraphen widmen wir uns zunächst Husserls Analysen von reellem Inhalt in Ideen $I$.

\section{c) Reelle Inhalte in Ideen I}

Husserl zufolge enthält die in phänomenologischer Reduktion betrachtete Baumwahrnehmung (1) stoffliche bzw. hyletische und (2) noetische Bestandteile als ihre reellen Inhalte.

(1) Stoffliche (oder hyletische) Bestandstücke der Wahrnehmung sind die Sinnesdaten wie Grün-Empfindung, Baumgestalt-Empfindung usw. Andererseits kann man auch das Wahrnehmungsnoema so beschreiben, dass der Baum, so wie er wahrgenommen wird, grün ist ${ }^{242}$; dabei spricht man eher von der „Farbe“ des Noema bzw. der noematischen Farbe. Husserl versucht, die Empfindungsfarbe von der noematischen Farbe zu unterscheiden:

\footnotetext{
${ }^{242}$ Vorausgesetzt, dass der grüne Baum wirklich existiert. Was passiert, wenn die Wahrnehmung nichtveridisch ist und der Gegenstand (z.B. die Farbe) nicht wirklich existiert? Darüber diskutieren wir im [\$42c] der vorliegenden Arbeit.
} 
Die Farbe des Baumstammes, rein als die wahrnehmungsmäßig bewußte, ist genau „dieselbe“ wie diejenige, die wir vor der phänomenologischen Reduktion als die des wirklichen Baumes nahmen ... Diese Farbe nun, in die Klammer gesetzt, gehört zum Noema. Nicht aber gehört sie als reelles Bestandstück zum Wahrnehmungserlebnis, obschon wir auch in ihm,,so etwas wie Farbe“ finden: nämlich die „Empfindungsfarbe“, das hyletische Moment des konkreten Erlebnisses, in welchen sich die noematische, bzw. ,objektive“ Farbe ,,abschattet"“ ${ }^{243}$

In diesem Passus beschreibt Husserl den Unterschied zwischen der hyletischen Farbe (Empfindungsfarbe) und der noematischen Farbe. Grob gesagt, die hyletische Farbe gehört zu der „,subjektiven“ Seite der phänomenologisch reduzierten Bewußtseinsphäre, während die noematische Farbe zu der „objektiven“ Seite gehört. In die Sprache der Logishcen Untersuchungen übersetzt, ist noematische Farbe eben das, was das Subjekt wahrnimmt (wenn die Farbe wirklich existiert ${ }^{244}$ ), im Vergleich dazu wird die hyletische Farbe nicht vom Subjekt gegenständlich wahrgenommen, sondern nur erlebt. ${ }^{245}$

Allerdings enthält der Passus zugleich eine Auskunft über den Zusammenhang zwischen der Empfindungsfarbe und der noematischen Farbe (Stichwort: Abschatten). Die Empfindungsfarbe hat die Funktion, die noematische Farbe darzustellen $(=\mathrm{zu}$ repräsentieren). ${ }^{246}$ Deshalb bezeichnet Husserl in der VI. LU die Empfindung auch als repräsentierenden Inhalt oder Repräsentant. ${ }^{247}$ Doch die Empfindung allein ist nicht imstande, diese repräsentierende Funktion zu übernehmen. Dafür muss sie von einem Aktcharakter (Apperzeption, Auffassung usw.) ${ }^{248}$ beseelt oder interpretiert werden-das

\footnotetext{
${ }^{243}$ Hua III/1: Ideen I, S.226.

${ }^{244}$ Was passiert, wenn die Wahrnehmung nichtveridisch ist und der Gegenstand (z.B. die Farbe) nicht wirklich existiert? Darüber diskutieren wir im $[\S 42 \mathrm{c}]$ der vorliegenden Arbeit.

${ }^{245}$ Hua XIX/1, S.358: „Beispielsweise ist also im Falle der äußeren Wahrnehmung das Empfindungsmoment Farbe, das ein reelles Bestandstück eines konkreten Sehens ... ausmacht, ebensogut ein ,,erlebter“ oder „bewußter Inhalt“, wie der Charakter des Wahrnehmens ... Dagegen ist dieser Gegenstand selbst, obgleich er wahrgenommen ist, nicht erlebt oder bewußt; und desgleichen auch nicht die an ihm wahrgenommene Färbung.“

${ }^{246}$ Abwechselnd verwendet Husserl die Terme „Darstellen“ und „Präsentieren“ abwechselnd, vgl. VI. LU, §22. Über die Pro- und Kontra-Argumente in Bezug auf Husserls Theorie von Empfindung vgl. Kenneth Williford, „Husserl's hyletic data and phenomenal consciousness“, in: Phenomenology and Cognitive Sciences 12, S. 501-519.

${ }^{247}$ Hua XIX/2: Logische Untersuchungen, S.609-610, 620.

248 „Die Wahrnehmungsvorstellung kommt dadurch zustande, daß die erlebte Empfindungskomplexion von einem gewissen Arkcharakter, einem gewissen Auffassen, Meinen beseelt ist ..." Hua XIX/1: Logische Untersuchungen,
} 
führt uns zu dem anderen reellen Inhalt, nämlich dem noetischen Bestandteil des Aktes.

(2) In den Logischen Untersuchungen ist dieser noetische Bestandteil als Aktcharakter, Auffassung, Apprehension usw. bezeichnet, in Ideen I wird er Noesis ${ }^{249}$ genannt. In unserem Beispiel der Farbenwahrnehmung ist die Noesis der entscheidende Faktor, der zur Intentionalität des Aktes beiträgt. Denn Husserl zufolge bezieht sich die Farbempfindung selber auf keine Gegenstände oder gegenständlichen Momente - sie übernimmt die Funktion, die gegenständliche Farbe darzustellen, nur dann, wenn sie durch die Noesis „,beseelt“" oder ,,gedeutet“ wird. In den Logischen Untersuchungen vergleicht Husserl die Beseelung der Empfindung durch die Auffassung mit der Beseelung des Ausdrucks durch die Bedeutungsintention ${ }^{250}$ : der Ausdruck als bloß physischer Gegenstand hat an und für sich keine Bedeutung und bezieht sich auf kein Gegenständliches; doch durch die Bedeutungsintention wird er als ein bedeutsamer Ausdruck interpretiert und er gewinnt somit Intentionalität auf ein Gegenständliches; in analoger Weise gewinnt die Farbempfindung die darstellende Funktion der gegenständlichen Farbe durch die Interpretation der Noesis.

Allerdings sollen wir dieser Analogie nicht $\mathrm{zu}$ viel zumuten. Die Bedeutungsintention ist fundiert auf einem Wahrnehmungsakt, der den Ausdruck als etwas Physisches zum Gegenstand hat. In diesem Sinne ist der Stoff der Auffassung ein sekundär bemerkter Gegenstand in der Fundierungseinheit von Wahrnehmung und Bedeutungsintention. Im Vergleich dazu ist in äußerer Wahrnemhung die Empfindung gar kein Gegenstand: Es ist nicht so, dass zuerst ein unterer Akt die Empfindung zum Gegenstand hat, und dann findet eine darauf fundierte Wahrnehmung statt, die den äußeren Gegenstand intendiert. Ausnahmsweise wird durch das Subjekt auf die Wahrnehmung reflektiert und ihre Empfindung zum Gegenstand gemacht. Doch der reflektierende Akt ist ein anderer Akt als die reflektierte Wahrnehmung. ${ }^{251}$

Die inneren Momente der Noesis werden wir unten ausführlich diskutieren. Zur Einführung des Begriffs Noesis genügt es hier, darauf hinzuweisen, dass der reelle

S.80. 395-400. Darüber hinaus vgl. Hua III/1: Ideen I, S.192, 227.

${ }^{249}$ Hua III/1: Ideen I, $\S 85,88,97$.

${ }^{250}$ Hua XIX/1: Logische Untersuchungen, §23.

${ }^{251}$ Ebd. 
Inhalt des Aktes eine stoffliche oder hyletische Komponente (Empfindung) und eine beseelende Komponente (die Noesis) enthält. In Husserls eignem Wort, ,,der Strom des phänomenologischen Seins hat eine stoffliche und eine noetische Schicht"(Hua III/1, S.196). Nach Husserl liegen die wichtigsten Analysen auf seiten der noetischen Schicht, denn die Noesis ist eben das, was den wesentlichen Charakter des Aktes, nämlich ,das Spezifische der Intentionalität hereinbringt“(a.a.O.,194), während die stoffliche Komponente nur als Stütze der Intentionalität fungiert. An einigen Stellen bezeichnet Husserl sogar die Einheit von Empfindung und Noesis, pars pro toto, und zwar unter Betonung der noetischen Komponente, auch als Noesis. ${ }^{252}$

\section{d) Einführung in die Konstitutionsanalysen und die Idee der transzendentalen Phänomenologie}

Die Noesis ist die reelle Komponente, die ,das Spezifische der Intentionalität hereinbringt", und zwar dadurch, dass sie die stoffliche Komponente (z.B. Empfindung) beseelt. Doch in einem Wahrnehmungsakt beseelt die Noesis die Empfindung nicht überhaupt, sondern durch einen bestimmten Auffassungssinn. Z.B. könnte dieselbe Farbempfindung als Farbe eines Baums oder Farbe einer Blume gedeutet werden. Der Auffassungssinn gehört zur noematischen Seite des Aktes und ist von Husserl auch als noematischer Sinn (im prägnanten Sinne ${ }^{253}$ ) bezeichnet. Der noematische Sinn entscheidet, welchen Gegenstand der Akt intendiert und als was er ihn intendiert. Doch es ist zu bemerken, dass der noematische Sinn nur eine Komponente (die KernKomponente) des vollen Noema ausmacht und dass für ihn nur ein Moment der vollen Noesis (die noetische Sinngebung ${ }^{254}$ ) im prägnanten Sinne zuständig ist. Von den verschiedenen Momenten der Noesis und ihren korrelativen Momenten in noematischer Hinsicht wird unten ausführlich gesprochen werden (in [\$§34ff.]). Zunächst wollen wir uns einer allgemeinen Charakterisierung der Beziehung zwischen Noesis und Noema

\footnotetext{
${ }^{252}$ Hua III/1, S.222: ,Wir haben der allgemeinen Herausarbeitung des Unterschiedes zwischen Noesis (d.i. dem konkret vollständigen intentionalen Erlebnis, bezeichnet unter Betonung seiner noetischen Komponenten) und Noema so große Sorgfalt angedeihen lassen ..."

${ }^{253}$ Der Terminus „,noematischer Sinn“ in einem laxen Sinne bezieht sich, wie Husserl ihn verwendet, auf das volle Noema.

${ }^{254}$ Hua III/1: Ideen I, S.194.
} 
widmen.

Etymologisch stammt das Wort Noesis von dem Verb voeĩv (denken) ab und

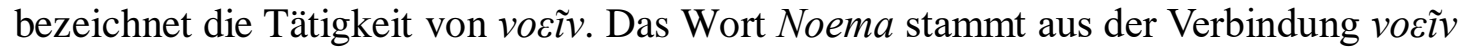
(v. denken) $+-\mu \breve{\alpha}$. Im Altgriechischen fügt man das Suffix $-\mu \breve{\alpha}$ einem verbalen Wortstamm hinzu, um das Produkt oder Objekt einer Tätigkeit zu bezeichnen. Dem Wortsinn nach ist also das Noema Produkt von Noesis; ${ }^{255}$ oder, mit Husserls eigenem Wort, die Noesis konstituiert das Noema. ${ }^{256}$ Den Konstitutionsbegriff verwendet Husserl sehr häufig in Ideen I, doch er thematisiert und erörtert diesen Begriff nicht systematisch. Eugen Fink ist der Meinung, dass der Konstitutionsbegriff einer der operativen Begriffe ist, die von Husserl verwendet werden, aber thematisch ungeklärt und gewissermaßen unbestimmt bleiben. ${ }^{257}$ Das heißt, dass Husserl den Konstitutionsbegriff aus seinem naiven Gebrauch (Zusammenstellen, Verfertigen, Herstellen, Produzieren usw.) aufnimmt und als Modell zur Beschreibung der Beziehung zwischen Noesis und Noema verwendet. ${ }^{258}$ Aber wie der Konstitutionsbegriff bei Husserl genau zu bestimmen ist und was ihn von seinem naiven Wortgebrauch unterscheidet, lässt sich nicht leicht entscheiden.

Dennoch ist es sicher, dass es sich bei Husserls Konstitutionsbegriff nicht um die beiden Extremfälle, nämlich totale Passivität ${ }^{259}$ von intentionalen Akten und Kreation der Gegenstände durch intentionale Akte handelt: Einerseits ist das „Auftauchen“ des Gegenstandes im Bewußtsein kein einfaches Vorkommnis, als ob das Bewußtsein nur ausgeruht auf den Gegenstand wartete. Ganz im Gegenteil vertritt Husserl die Ansicht, dass das Bewußtsein etwas aktiv leisten muss, damit der Gegenstand in ihm gegeben werden kann. ${ }^{260}$ Z.B. muss das Bewußtsein den Gegenstand in einem bestimmten

\footnotetext{
${ }^{255}$ Elisabeth Ströker, Husserls transzendentale Phänomenologie, Vittorio Klostermann, Frankfurt am Main 1987, S.109, Fußnote 49.

${ }^{256}$ Ebd., S.118: „Den Noesen eignet demnach eine gewisse Produktivität; ihre Konstitution ist Produktion. Ein Konstituiertes ist für Husserl fraglos ein Produziertes des transzendentalen Bewußtseins.“

${ }^{257}$ Eugen Fink, ,Operative Begriffe in Husserls Phänomenologie“, in: Zeitschrift für philosophische Forschung, Bd. 11, H.3 (Jul.-Sep., 1957), Vittorio Klostermann, S.334ff.

${ }^{258} \mathrm{Ebd}$.

${ }^{259}$ Der Begriff von Passivität wird hier nur in einem sehr laxen metaphorischen Sinne verwendet, um anzudeuten, dass das Subjekt im Bewußtseinsleben verschiedene Geistestätigkeiten aktiv ausübt und nicht nur Reize passiv rezeptiert, um das Gegebensein des Gegenstandes oder Noema zu organisieren. Aber man kann auch diesen Begriff im Zusammenhang mit dem Passivitätsbegriff, den ich im III. Abschnitt der vorliegenden Arbeit (§§57-58) diskutieren werde, phänomenologisch näher charakterisieren. Dabei ist mit Passivität eine besondere Stufe des erkenntniserzeugenden Prozesses gemeint.

${ }^{260}$ Elisabeth Ströker, Husserls transzendentale Phänomenologie, S.116-117.
} 
Modus der Aufmerksamkeit betrachten, was schon zu einer seiner aktiven Leistungen zählt und einen Aspekt der Noesis bestimmt. Andererseits darf man die konstitutiven Leistungen des Bewußtseins nicht so stark interpretieren, als ob die Konstitution eine Erschaffung oder Kreation der Gegenstände und der Welt wäre. Wie Ströker bemerkt, „Die Noesen erzeugt nicht Sein, sie setzen Sein jeweils in einem bestimmten Sinn. Dabei ist nicht die Frage, woher das Sein stamme ... Sie hätte in der Husserlschen Philosophie gar keinen Ort.“ ${ }^{261}$ Die Konstitution bewirkt nicht das Sein des Gegenstandes, sie bewirkt höchstens das Gegenstandsein des Seienden. ${ }^{262}$ Die Frage, wie sich der wirklich seiende Gegenstand zu dem Noema verhält, wird uns unten noch beschäftigen.

Eine systematische Erforschung des Konstitutionsbegriffs würde die Grenzen der vorliegenden Arbeit überschreiten. Aufgabe dieses Kapitels und des nächsten Kapitels ist, wie es bei Husserl der Fall ist, mit dem operativen Konstitutionsbegriff die verschiedenen noetischen Momente und ihre korrelativen noematischen Momente sowie deren konstitutive Beziehungen $\mathrm{zu}$ beschreiben. Dafür genügt es, darauf hinzuweisen, dass eine Bestimmung von Husserls Konstitutionsbegriff die beiden oben erwähnten Extremfälle ausschließt. Um vorwärtsgehen zu können, müssen wir die etwas metaphorische Unbetimmtheit des Konstitutionsbegriffs aushalten. ${ }^{263}$

Genauer gesprochen enthält das Noema verschiedene Momente, für deren Konstitution jeweilige noetische Momente zuständig sind. Zum Beispiel ist der noetische Setzungscharakter zuständig für die Konstitution des noematischen Seinscharakters (vg1.[§37]). Doch bei einer Konsitutionsanalyse von einzelnem Akt wollen wir es nicht bewenden lassen, denn konstitutive Leistungen können auch durch die Zusammenarbeit von mannigfaltigen Akten vollzogen werden: neben den jeweiligen Noemata wird auch „der Gegenstand“ (Gegenstand-inAnführungszeichen) ${ }^{264}$, der durch mannigfaltige Noemata identisch durchdringt,

\footnotetext{
${ }^{261}$ Ebd., S.118.

${ }^{262}$ Eugen Fink, „Operative Begriffe in Husserls Phänomenologie“, S. 332.

${ }^{263}$ Der Konstitutionsbegriff ist für Eugen Fink eng verbunden mit Husserls Begriff von Gegenstandssinn. Unter Gegenstandssinn verstehe ich eben Noema. In diesem Sinne ist die Erörterung des Noemabegriffs zugleich eine Erörterung des Konstitutionsbegriffs. In folgenden Paragraphen werde ich mich eben mit dem Noemabegriff beschäftigen.

${ }^{264}$ Die Einfügung der Anführungszeichen lässt sich durch Husserls Begriff des bestimmbaren X erklären. Darüber
} 
konstituiert. Daher muss eine vollständige Konstitutionsanalyse nicht nur die konstitutiven Leistungen von einzelnen Akten, sondern auch die synthetische Leistung von mannigfaltigen Akten berücksichtigen. Grob gesagt ist Synthese im Kontext der Konstitutionsanalyse ein Bewußtseinsprozess, in dem Erlebnisse sich zu einer Einheit vereinigen, die ,ein und denselben Gegenstand“ konstituiert und intendiert. ${ }^{265}$ Doch die beiden Aufgaben sind nicht zusammenhangslos, denn die konstitutiven Leistungen von einzelnen Akten haben die Funktion, zur Konstitution ,ein und desselben Gegenstandes“ beizutragen. Im [§86] der Ideen I fasst Husserl die beiden Aufgaben unter dem Gesichtspunkt „der funktionellen Probleme“ zusammen:

Doch die allergrößten Probleme sind die funktionellen Probleme, bzw. die der „Konstitution der Bewußtseinsgegenständlichkeiten“. Sie betreffen die Art, wie z.B. hinsichtlich der Natur, Noesen, das stoffliche beseelend und sich zu mannigfaltig-einheitlichen Kontinuen und Synthesen verflechtend, Bewußtsein von Etwas so zustande bringen, daß objektive Einheit der Gegenständlichkeit sich darin einstimmig „bekunden“, ,,ausweisen“ und „vernünftig“ bestimmen lassen kann.

Der Gesichtspunkt der Funktion ist der zentrale der Phänomenologie, die von ihm ausstrahlenden Untersuchungen umspannen so ziemlich die ganze phänomenologische Sphäre, und schließlich treten alle phänomenologischen Analysen irgendwie in ihren Dienst als Bestandstücke oder Unterstufen. An die Stelle der an den einzelnen Erlebnissen haftenden Analyse und Vergleichung, Deskription und Klassifikation, tritt die Betrachtung der Einzelheiten unter dem ,teleologischen“ Gesichtspunkt ihrer Funktion, „synthetische Einheit“" möglich zu machen. ${ }^{266}$ (meine Herv.)

Dem Zitat zufolge bildet die Analyse der konstitutiven Leistungen von einzelnen Akten die Unterstufe ${ }^{267}$ für die der synthetisch-konstitutiven Leistungen von mannigfaltigen

\footnotetext{
vgl. [ $\S 40-42]$ der vorliegenden Arbeit.

${ }^{265}$ Zur näheren Verständigung des Begriffs synthetisch vgl. Hua III/1, §118 und Hua I, §§17-18; dazu vgl. auch David Bell, Husserl, S.176.

${ }^{266}$ Hua III/1: Ideen I, §86.

${ }^{267}$ Der Schwerpunkt der punktuellen Analysen (= Analysen der konstitutiven Leistungen von einzelnen Akten) ist,
} 
Akten. Denn das konstituierte Noema eines einzelnen Aktes dient ,teleologisch“ der Konstitution „ein und desselben Gegenstandes“ (Gegenstand-in-Anführungszeichen), der auch von anderen Akten intendiert werden kann. Metaphorisch gesagt ist die konstitutive Leistung eines einzelnen Aktes ein Querschnitt der synthetischkonstitutiven Leistung der mannigfaltigen Akte, in denen ,ein und derselbe Gegenstand“"konstitutiert wird.

Der Unterschied zwischen den Konstitutionsanalysen von einzelnen Akten und den Konstitutionsanalysen unterm funktionellen Gesichtspunkt hängt mit einer Zweideutigkeit des Noemabegriffs zusammen. Rudolf Bernet hat bemerkt, Husserls Noemabegriff sei mit einer Äquivokation behaftet: Der Noemabegriff beziehe sich einerseits auf das, was in der Leistung eines einzelnen Aktes konstituiert wird (=das jeweilige Noema oder das jeweilige noematische Korrelat, wie Bernet es nennt), andererseit auch auf den in synthetisch verbundenen Akten konstituierten Gegenstandin-Anführungszeichen. ${ }^{268}$ Doch diese Äquivokation ist nicht gefährlich, solange wir den Zusammenhang zwischen der konstitutiven Leistung eines einzelnen Aktes und der der synthetischen verbundenen Akte berücksichtigen: sowohl das jeweilige Noema als auch der Gegenstand-in-Anführungszeichen sind konstituierte noematische Gegebenheiten, während das erstere ein Querschnitt des letzteren bildet; das erstere untersuchen die Konstitutionsanalysen des einzelnen Aktes, und das letztere untersuchen die Konstitutionsanalysen der synthetisch verbundenen Akte; terminologisch bezeichnen wir die erstere Konsitutionsanalyse als punktuelle (noetisch-noematische) Konstitutionsanalyse, während die letztere als dynamische (noetisch-noematische) Konstitutionsanalyse zu bezeichnen ist.

In folgenden Paragraphen des Kapitels beschränken wir uns auf die punktuelle Konstitutionsanalyse, die sich hauptsächlich an der konstitutiven Leistung eines

\footnotetext{
wie unten ausgeführt, die verschiedenen noetisch-noematischen Momente zu unterscheiden, wie die Anatomie die inneren Organe des Körpers unterscheidet. Dann fragt man, wie diese noetisch-noematischen Momente kooperieren, um die zentrale Funktion auszuüben, eine Gegenstandseinheit zu konstituieren. In analoger Weise fragt man, ausgehend von der Anatomie, wie die Organe zusammenarbeiten, um den Körper am Leben zu erhalten. Die punktuellen Analysen sind Unterstufe für die dynamischen Analysen (= Analysen der synthetisch-konstitutiven Leistungen von mannigfaltigen Akten) wie die Anatomie ist Unterstufe für die Physiologie.

${ }^{268}$ Rudolf Bernet, „Husserls Begriff des Noema““, in: Husserl-Ausgabe und Husserl-Forschung, hrsg. von Samuel Ijsseling, Kluwer Academic Publishers, Dordrecht/Boston/London 1990, S.67, 70.
} 
einzelnen Aktes orientiert. Dann werden wir uns im nächsten Kapitel mit der dynamischen Konstitutionsanalyse, die die synthetische Einheit von Akten sowie den in dieser Einheit konstitutierten Gegenstand-in-Anführungszeichen betrachtet, beschäftigen.

Einige terminologische Anmerkungen: sowohl die punktuelle als auch die dynamische Konstitutionsanalyse untersucht das phänomenologisch reduzierte Bewußtsein und gehört zum Bereich der Untersuchungen von reiner Phänomenologie. Die konstituierende Seite des phänomenologisch reduzierten Bewußtseins, nämlich die Noesis, bildet ein reelles Bestandstück des Bewußtseins. Im Gegensatz dazu ist das Noema (das jeweilige Noema und der Gegenstand-in-Anführungszeichen), als intentionaler Inhalt des Bewußtseins, nicht reell in ihm enthalten. In diesem Sinne ist das Noema etwas der Noesis Transzendentes ${ }^{269}$; und die reine Phänomenologie untersucht, wie das Bewußtsein etwas ihm Transzendentes konstituiert. Daher bezeichnet Husserl die phänomenologische Reduktion auch als transzendentale Reduktion und die reine Phänomenologie als transzendentale Phänomenologie. ${ }^{270}$

\section{§33. Das Prinzip des noetisch-noematischen Parallelismus}

Bevor wir in die Konstitutionsanalysen eingehen, möchte ich zuerst das Prinzip formulieren, nach dem Husserl die Konstitutionsanalysen vollzieht. In Ideen I schreibt Husserl:

Überall entspricht den mannigfaltigen Daten des reellen, noetischen Gehaltes eine Mannigfaltigkeit in wirklich reiner Intuition aufweisbarer Daten in einem korrelativen ,,noematischen Gehalt“, oder kurz im „Noema“.

Kein noetisches Moment ohne ein ihm spezifisch zugehöriges noematisches Moment, so lautet das sich überall bewährende Wesensgesetz.

Es gilt dann aber auch, daß die Wesen Noema und Noesis voneinander unabtrennbar sind: Jede niederste Differenz auf der noematischen Seite weist

\footnotetext{
${ }^{269}$ Außerdem ist der Gegenstand des Bewußtseins in einem anderen Sinn transzendent, indem er dem Bewußtsein nur unter bestimmten kognitiven Perspektiven ,einseitig“, aber niemals ,,allseitig“" gegeben ist. Vgl. [§41-43] der vorliegenden Arbeit; dazu auch David Bell, Husserl, S.200.

${ }^{270}$ Hua III/1: Ideen I, S.198, 228.
} 
eidetisch zurück auf niederste Differenzen der noetischen. (Hua III/1, 203, 215, 296)

Den Zitaten zufolge gibt es zwischen Noesis und Noema eine Korrelation, die wir schon im Vorbeigehen (im [§32d]) erwähnt haben. Diese Korrelation heißt, dass das Noema verschiedene Momente enthält, für deren jeweilige Konstitution jeweils ein noetisches Moment zuständig ist. Deshalb spricht Husserl vom „Parallelismus noetischer und entsprechender noematischer Charaktere"(a.a.O., 232). Wir können das Prinzip des noetisch-noematischen Parallelismus oder mit Husserls eigenem Wort, das Gesetz der Korrelation von Noesis und Noema (a.a.O., 282) wie folgt formulieren:

\section{(Das Prinzip des noetisch-noematischen Parallelismus)}

Jedem noematischen Moment (oder jedem noematischen Charakter) entspricht ein noetisches Moment (oder ein noetischer Charakter), das für seine Konstitution zuständig ist. Und umgekehrt: jedem noetischen Moment (oder jedem noetischen Charakter) entspricht ein noematisches Moment (oder ein noematischer Charakter), das von ihm konstituiert wird.

Dieses Prinzip befolgt Husserl wohl bewußt in seinen Konstitutionsanalysen ${ }^{271}$ das zeigt sich darin, dass die Stichworte der Konstitutionsanalysen immer Begriffspaare wie noetische Sinngebung-noematischer Sinn, noetischer Setzungscharakternoematischer Seinscharakter usw. sind. In Ideen I beschäftigt Husserl sich hauptsächlich mit den jenigen Begriffspaaren, die allgemein auf alle Akte anwendbar sind, egal, was für ein Gegenstand (Ding, Mensch oder Kunstwerk) in diesen Akten intendiert wird. ${ }^{272}$ Nun wollen wir uns diesen Begriffspaaren und den ihnen zugehörigen Konstitutionsanalysen zuwenden.

\footnotetext{
${ }^{271}$ Ebd., S.211: ,Wir werden die verschiedenen Bewußtseinsarten mit ihren vielfältigen noetischen Charakteren der Reihe nach analysieren und sie nach den noetisch-noematischen Parallelen durchforschen.“ Ebd., S.232: „Wir blicken uns in der Bewußtseinssphäre noch etwas weiter um und versuchen, an den hauptsächlichsten Bewußtseinsweisen die noetisch-noematischen Strukturen kennen zu lernen. In der wirklichen Nachweisung versichern wir uns zugleich schrittweise der durchgängigen Geltung der fundamentalen Korrelation zwischen Noesis und Noema."

${ }^{272}$ Akte, die verschiedene Gegenstandsarten intendieren, haben verschiedene Konstitutionsarten. Dementsprechend haben sie auch verschiedene noetisch-noematische Momente. (Z.B. die Wahrnehmung eines Menschen vs. die Wahrnehmung eines Steins). In Ideen I beschäftigt Husserl sich hauptsächlich mit den noetisch-noematischen Momenten oder Charakteren, die allgemein bei der Konstitution von allen Akten eine Rolle spielen.
} 


\section{$\S 34$. Noetische Sinngebung und noematischer Sinn}

In [§32c-d] haben wir den Auffassungscharakter der Noesis diskutiert, und zwar unter Betonung ihrer Sinngebung-Funktion: In unserem Beispiel der Baumwahrnehmung fasst die Noesis die stofflich-reelle Komponente, nämlich die Baumempfindungen auf, damit der Akt einen Gegenstand unter dem noematischen Sinn „,blühender Baum“ wahrnehmungsmäßig intendiert. Den noematischen Sinn bezeichnet Husserl auch als Sinn, ,intentionales Objekt“(eine unglückliche Bezeichnung), oder gegenständlichen Sinn usw. ${ }^{273}$ Husserl zufolge lässt sich diese an dem Wahrnehmungsbeispiel erkannte noetisch-noematische Korrelation, die durch das Begriffspaar noetische Sinngebung-noematischer Sinn gekennzeichnet wird, auf alle intentionalen Akte verallgemeinern:

Ähnlich wie die Wahrnehmung hat jedes intentionale Erlebnis - eben das macht das Grundstück der Intentionalität aus - sein ,,intentionales Objekt“, d.i. seinen gegenständlichen Sinn. Nur in anderen Worten: Sinn zu haben, bzw. etwas „im Sinne zu haben“, ist der Grundcharakter alles Bewußtseins, das darum nicht nur überhaupt Erlebnis, sondern sinnhabendes, ,,noetisches“ “ ist. ${ }^{274}$ Husserl vertritt also die Ansicht, dass jedes intentionale Erlebnis auf der noetischen Seite eine Sinngebung vollzieht und auf der noematischen Seite einen noematischen Sinn konstituiert.

Tatsächlich ist der Begriff der noetischen Sinngebung, die zum noetisch-reellen Inhalt des Aktes gehört, ein Nachfolger des Begriffs von reeller Materie in den Logischen Untersuchungen, korrelativ ist der Begriff des noematischen Sinnes, der zum noematisch-intentionalen Inhalt des Aktes gehört, ein Nachfolger des Begriffs von idealer Materie (vgl. Hua III/1, S.298). Die (reelle bzw. ideale) Materie entscheidet nicht nur, welchen Gegenstand der betreffende Akt intendiert, sondern auch, als was er ihn intendiert - dasselbe gilt auch für die noetische Sinngebung und den noematischen Sinn. Der Unterschied der beiden Begriffspaare besteht darin, dass die reelle Materie zu der idealen im Einzelfall-Spezies-Verhältnis steht, während sich die Sinngebung und

\footnotetext{
${ }^{273}$ Hua III/1: Ideen I, S.206.
}

${ }^{274}$ Ebd. 
der noematische Sinn in der Konstitutionsbeziehung befinden. Husserl bevorzugt das neue Begriffspaar (Sinngebung - noematischer Sinn), denn er glaubt, dass „die Einseitigkeit der noetischen Blickrichtung“, die in dem alten Begriffspaar (reelle Materie - ideale Materie) herrscht, durch das neue Paar überwunden wird (a.a.O.). Es ist $\mathrm{zu}$ fragen, ob das Einzelfall-Spezies-Modell und das Konstitutionsmodell kompatibel sind. Kann der noematische Sinn nicht zugleich eine Spezies der noetischen Sinngebung sein? Es gibt Fälle, wo eine ähnliche Kompatibilität möglich ist: wenn ein Reisender zum ersten Mal Heimweh hat, dann kontituiert er die Heimweh-Idee, die zugleich die Spezies seines Heimweh-Erlebnisses ist. Ich sehe keinen Grund, die Kompatibilität der beiden Modelle ohne weiteres abzulehnen. ${ }^{275}$

Husserl zufolge bildet die Sinngebung das fundamentale Moment der Noesis (a.a.O., 194), korrelativ ist der noematische Sinn das zentrale Moment des Noema und somit gilt als „,noematischer Kern“. Doch die Sinngebung und der noematische Sinn erschöpfen nicht die Noesis und das Noema. Husserl schreibt:

Freilich erschöpft, was in unserer Beispielsanalyse als „Sinn“ sich abgehoben hat, nicht das volle Noema; dementsprechend besteht die noetische Seite des intentionalen Erlebnisses nicht bloß aus dem Moment der eigentlichen „Sinngebung“, dem speziell der „Sinn“ als Korrelat zugehört. Es wird sich bald zeigen, daß das volle Noema in einem Komplex noematischer Momente besteht, daß darin das spezifische Sinnesmoment nur eine Art notwendiger Kernschicht bildet, in der weitere Momente wesentlich fundiert sind, die wir nur darum ebenfalls, aber in extendiertem Sinn, als Sinnesmomente bezeichnen durften. (Hua III/1, 206; meine Herv.)

Wir merken hierdurch, daß wir innerhalb des vollen Noema ... wesentlich verschiedene Schichten sondern müssen, die sich um einen zentralen „Kern“, um den puren ,gegenständlichen Sinn“ gruppieren. (a.a.O., 210; meine Herv.) Solcher immer am noematischen Kern haftenden Charaktere gibt es aber noch ganz andere, und der Arten, wie sie ihm zugehören, sehr verschieden. Sie

\footnotetext{
275 Damit im Zusammenhang steht Beyers Diskussion über Husserls zwei Bedeutungsbegriffe, die wir unten im [§43] besprechen werden. Vgl. Christian Beyer, Intentionalität und Referenz, S.106-108.
} 
ordnen sich grundverschiedenen Dimensionen der Charakterisierung ... Dabei ist die Sachlage die, daß allzeit und notwendig ein noematischer Kern, ein „Gegenstandsnoema“, bewußt ist, das irgendwie charakterisiert sein muß, und zwar nach diesen oder jenen (sich ihrerseits ausschließenden) Differenzen aus jeder Gattung. (a.a.O., 238; meine Herv.)

Das Noema besteht, so Husserl, in ,einem Komplex noematischer Momente“, wobei der noematische Sinn nur die „notwendige Kernschicht“ bildet, in der die anderen noematischen Momente (als weitere Schichten oder Charaktere des Noema) fundiert sind. Diese anderen noematischen Momente charakterisieren das volle Noema nach verschiedenen Dimensionen. Nach dem Prinzip des noetisch-noematischen Parallelismus können wir sagen, dass die Noesis auch in einem Komplex noetischer Momente besteht, wobei die Sinngebung nur die Kernschicht bildet, in der andere noetische Momente (als weitere Schichten oder Charaktere der Noesis) fundiert sind. Diese anderen Momente charakterisieren die volle Noesis nach verschiedenen Dimensionen.

Diese anderen noetisch-noematischen Momente beschäftigen uns in den folgenden Paragraphen.

\section{§35. Noetischer Repräsentationscharakter und noematischer}

\section{Anschauungscharakter}

Akte verschiedener Arten können denselben noematischen Sinn haben, wobei ihre Noemata voneinander verschieden sind. Husserls Beispiel:

Jedem dieser Erlebnisse [Wahrnehmung, Erinnerung und Phantasie usw. Anm.d.Verf.] „wohnt“ ein noematischer Sinn [= das volle Noema Anm.d.Verf.] ${ }^{276}$ „ein“, und wie immer dieser in verschiedenen Erlebnissen verwandt, ja ev. einem Kernbestande nach wesensgleich sein mag, er ist jedenfalls in artverschiedenen Erlebnissen ein verschiedenartiger, das gegebenenfalls Gemeinsame ist zum mindesten anders charakterisiert, und das

\footnotetext{
${ }^{276}$ Aus dem Kontext geht hervor, dass Husserl hier mit „,noematischer Sinn“, pars pro toto, das volle Noema meint.
} 
in Notwendigkeit. Es mag sich überall um einen blühenden Baum handeln, und überall mag dieser Baum in solcher Weise erscheinen, daß die getreue Beschreibung des Erscheinenden als solchen notwendig mit denselben Ausdrücken erfolgt. Aber die noematischen Korrelate sind darum doch wesentlich verschiedene für Wahrnehmung, Phantasie, bildliche Vergegenwärtigung, Erinnerung usw. Einmal ist das Erscheinende charakterisiert als ,leibhafte Wirklichkeit“, das andere Mal als Fiktum, dann wieder als Erinnerungsvergegenwärtigung usw.

Das sind Charaktere, die wir am Wahrgenommenen, Phantasierten, Erinnerten usw. als solchem - am Wahrnehmungssinn, am Phantasiesinn, Erinnerungssinn - als ein unabtrennbares vorfinden, und als notwendig Zugehöriges in Korrelation zu den betreffenden Arten noetischer Erlebnisse. ${ }^{277}$

Das Subjekt kann also verschiedenartige Akte vollziehen, deren noematischer Sinn (im strengen Sinne) mit demselben Ausdruck wie „,blühender Baum“ zu beschreiben und somit identisch ist. Doch dieser Sinn ist in ihnen mit verschiedenen Anschauungscharakteren verbunden: In Wahrnehmung ist er mit dem Anschauungscahrakter der Gegenwärtigung, durch den der Gegenstand gegenwärtig oder originär intendiert wird, in Erinnerung mit dem der erinnerungsmäßgien Vergegenwärtigung, durch den der Gegenstand im Modus ,gegenwärtig gewesen" intendiert wird, in Phantasie mit dem der phantasiemäßigen Vergegenwärtigung usw. Deshalb lautet ein Passus, der sich auf die oben zitierten Textstellen beruft, wie folgt:

Wir erinnern zunächst an jenen ,gegenständlichen Sinn“, der sich uns oben ${ }^{278}$ durch Vergleichung von Noemen verschiedenartiger Vorstellungen, von Wahrnehmungen, Erinnerungen, Bildvorstellungen u. dgl. ergab, als ein mit lauter objektiven Ausdrücken zu Beschreibendes und sogar wechselseitig mit identischen [Ausdrücken] ... sich ... darstellt. Gegenüber dem

\footnotetext{
${ }^{277}$ Hua III/1: Ideen I, S.210.

${ }^{278}$ Hier wird Husserls Fußnote, die sich auf die oben zitierten Textstellen beruft, hinzugefügt. Hier meint Husserl mit „gegenständlicher Sinn“ den noematischen Sinn im strengen Sinne.
} 
identischen „erscheinenden Baum als solchen“ mit dem identischen „objektiven“ Wie des Erscheinens verbleiben die von Anschauungsart $\mathrm{zu}$ Anschauungsart und nach sonstiger Vorstellungsart wechselnden Unterschiede der Gegebenheitsweise.

Jenes Identische ist einmal „originär“ bewußt, das andere Mal „erinnerungsmäßig“ bewußt, dann wieder ,,bildmäßig“ usw. Damit aber bezeichnen sich Charaktere am ,erscheinenden Baum als solchen“, vorfindlich in der Blickrichtung auf das noematische Korrelat und nicht in derjenigen auf das Erlebnis und seinen reellen Bestand. ${ }^{279}$

Diese mit den anschaulichen Gegebenheitsweisen des Gegenstandes variierenden noematischen Charaktere möchte ich als (noematische) Anschauungscharaktere bezeichnen. Nach dem Prinzip des noetisch-noematischen Parallelismus sollen korrelativ noetische Momente bestehen, die die Arten von anschaulichen Akten bestimmen und somit für Konstitution der noematischen Anschauungscharaktere zuständig sind. Diese noetischen Momente hat Husserl tatsächlich schon in der VI. LU, obwohl in einer „Einseitigkeit der noetischen Blickrichtung“ (mangels der Unterscheidung zwischen Noesis und Noema), unter dem Titel Repräsentation studiert. ${ }^{280}$ Terminologisch möchte ich sie noetische Repräsentationscharaktere nennen.

Oben diskutieren wir nur Beispiele von anschaulichen Akten, deren Repräsentationscharaktere intuitiv sind. Doch Husserl spricht auch von Repräsentation von signitiven Akten wie leeren Bedeutungsintentionen, die er signitive Repräsentation nennt. Eine leere Bedeutungsintention kann auch denselben noematischen Sinn (wie „der blühende Baum“) mit einer Wahrnehmung teilen, wobei der Sinn nicht mit dem noematischen Anschauungscharakter von Gegenwärtigung oder „originär“, sondern mit einem noematischen Charakter, der angesichts des intendierten Gegenstandes (des Baums) völlig unanschaulich ist, verbunden wird. Zur einheitlichen Terminologie wollen wir diesen (noematischen) null-anschaulichen Charakter auch als Anschauungscharakter bezeichnen, wie man manchmal die Zahl 0 auch zu den

\footnotetext{
${ }^{279}$ Hua III/1: Ideen I, S. 233.

${ }^{280}$ Hua XIX/2: Logische Untersuchungen, §25-26.
} 
natürlichen Zahlen zuordnen will. ${ }^{281}$

Auf diese Weise gewinnen wir, neben dem Paar noetische Sinngebung noematischer Sinn, das Begriffsduo noetischer Repräsentationscharakter noematischer Anschauungscharakter, die sowohl auf anschauliche Akte als auch auf nicht-anschauliche Akte anwendbar ist. Ein und derselbe noematische Sinn, wie gesagt, kann mit verschiedenen noematischen Anschauungscharakteren verbunden werden; auf noetischer Seite wird die Sinngebung, die ein und denselben noematischen Sinn konstituiert, mit verschiedenen Repräsentationscharakteren verbunden. Nach dem Prinzip des noetisch-noematischen Parallelismus ist der noetische Repräsentationscharakter zuständig für die Konstitution des noematischen Anschauungscharakters.

\section{§36. Noetischer Aufmerksamkeitscharakter und noematischer Auffälligkeitscharakter}

Bei einer anderen noetisch-noematischen Begriffsbildung handelt es sich um den Aufmerksamkeitsmodus des Aktes. Bei der Wahrnehmung des vor mir liegenden Papiers bin ich ihm aufmerksam zugewendet, ich erfasse es. Mit den Termen ,Zuwenden“, ,Erfassen“ meint Husserl „Herausfassen“:

Das Erfassen ist ein Herausfassen, jedes Wahrgenommene hat einen Erfahrungshintergrund. Rings um das Papier liegen Bücher, Stifte, Tintenfaß usw., in gewisser Weise auch ,wahrgenommen“, perzeptiv da, im ,Anschauungsfelde“, aber während der Zuwendung zum Papier entbehrten sie jeder, auch nur sekundären Zuwendung und Erfassung. ${ }^{282}$

Husserls Meinung nach hebt sich das Papier bei der Wahrnehmung durch die aufmerksame Zuwendung des Subjekts von seinem Hintergrund ab. Man kann sagen, dass der erfasste Gegenstand auffällig dasteht, während die hintergründlichen Gegenstände nur unauffällig vorhanden sind. Allerdings sind die unauffälligen Gegenstände nicht nichts, sondern bereits bewußt. Korrelativ ist das hintergründliche

\footnotetext{
${ }^{281}$ In VI. LU umfasst der Begriff repräsentierender Inhalt sowohl intuitive als auch signitive Inhalte. Ebd., S.620.

${ }^{282}$ Hua III/1: Ideen I, S.71.
} 
Bewußtsein auch, wie das erfassende, Bewußtsein von etwas und somit untersteht der Gattung intentionale Erlebnisse oder intentionale Akte im weitesten Sinne (Hua III/1, 74). Der Unterschied besteht darin, dass das erfassende Bewußtsein im Modus aktueller Zuwendung (oder Modus der Aktualität), während das hintergründliche Bewußtsein im Modus der Inaktualität stattfindet (a.a.O., 72). Terminologisch will Husserl die Cartesianischen Reden von cogito und cogitatio ausschließlich für das erfassende Bewußtsein (,Akte im engeren Sinn“ ${ }^{283}$ ) vorbehalten. Wenn er von dem hintergründlichen Bewußtsein spricht, will er den Beisatz ,,inaktuell“ hinzufügen und die Rede inaktuelle cogitatio verwenden (a.a.O., 73).

Drei Punkte sind zu ergänzen. (1) Der Begriff von Aktualität/Inaktualität erlaubt Graduierung: Der „Blickstrahl“ der Aufmerksamkeit kann dem Papier primär (wenn ich mich hauptsächlich für das Papier interessiere), sekundär (wenn ich mich hauptsächlich für den Tisch interessiere und das Papier nur im Vorbeigehen beachte), und bis auf in einem sehr schwachen Grad (wenn das Papier einfach im Hintergrund steht) zugewendet werden. Der Modus der Inaktualität lässt sich gleichsam in dem sehr niedertourigen Bereich des Drehzahlmessers von Aufmerksamkeit lokalisieren. (2) Der Hintergrundbegriff beschränkt sich nicht auf räumlichen Hintergrund. Wenn ich z.B. auf dem Papier einen arithmetischen Beweis schreibe, dann bin ich primär den arithmetischen Gegenständen (Zahlen) zugewendet und das Papier ist hintergründlich bewußt, wobei die arithmetischen Gegenstände und das Papier nicht in demselben Raum stehen. (3) Husserl spricht auch von den Modifikationen, die den Akt von einem Modus der Aufmerksamkeit in einen anderen Modus überführen. Z.B. ist es möglich, dass ich einen primär bemerkten Gegenstand nachher mit weniger Aufmerksamkeit oder sogar im Modus der Inaktualität bemerken werde (a.a.O., 72).

Terminologisch wollen wir das Begriffspaar noetischer Aufmerksamkeitscharaker - noematischer Auffälligkeitscharakter einführen, um die noetisch-noematische Schicht, die solche Modifikationen betreffen, zu kennzeichnen. Husserl zufolge können solche Modifikationen der Aufmerksamkeit auch „unbeschadet des identischen

\footnotetext{
${ }^{283}$ Ebd., S.264.
} 
noematischen Kerns ${ }^{\text {“284 }}$ stattfinden. Auf der noetischen Seite kann ein und dieselbe noetische Sinngebung in verschiedenen Modi der Aufmerksamkeit, d.h. in verschiedenen noetischen Aufmerksamkeitscharakteren stattfinden; korrelativ kann auf der noematischen Seite ein und derselbe noematische Sinn mit verschiedenen Modi der Auffälligkeit, d.h. mit verschiedenen noematischen Auffälligkeitscharakteren verbunden werden. Nach dem Prinzip des noetisch-noematischen Parallelismus ist der noetische Aufmerksamkeitscharakter zuständig für die Konstitution des noematischen Auffälligkeitscharakters.

\section{§37. Noetischer Glaubenscharakter und noematischer Seinscharakter}

In der $V . L U$ klassifiziert Husserl die objektivierenden Akte, wie wir schon im [§4] des I. Abschnitts ausgeführt haben, in setzende und nicht-setzende. Mangels des Begriffsduos von Noesis/Noema wird diese Klassifikation in den Logischen Untersuchungen nur für eine Unterscheidung von Aktqualität gehalten und deshalb, wie Tugendhat bemerkt, ,einseitig noetisch verstanden“285. In Ideen I wird sie im Rahmen der noetisch-noematischen Analysen erneut behandelt und in den folgenden Apekten weiter entwickelt: (1) Mittels des Begriffs von Noesis und Noema wird der Glaubenscharakter des wirklich-Seiendes Setzens sowohl auf der noetischen Seite als auch auf der noematischen Seite betrachtet; (2) Neben dem Charakter des wirklichSeiendes Setzens diskutiert Husserl auch andere Glaubenscharaktere; (3) Die anderen Glaubenscharaktere werden als doxische Modifikationen des wirklich-Seiendes Setzens betrachtet; (4) Die Neutralitätsmodifikation, die einen Glaubenscharakter in einen nicht-setzenden Charakter umverwandelt, lässt sich nicht nur auf den Charakter des wirklich-Seiendes Setzens, sondern auch auf andere Glaubenscharaktere anwenden.

(1) Im Rahmen der Konstitutionsanalysen kann man den setzenden Charakter eines objektivierenden Aktes, der seinen Gegenstand als wirklich-seiend setzt, auch

\footnotetext{
${ }^{284}$ Ebd., S.213: ,Andererseits ist es klar, daß diese Modifikationen [von Aufmerksamkeit - Anm. d. Verf.] nicht nur solche des Erlebnisses selbst in seinem noetischen Bestande sind, sondern daß sie auch seine Noemen angreifen, daß sie auf noematischer Seite - unbeschadet des identischen noematischen Kerns - eine eigene Gattung von Charakterisierungen darstellen."

${ }^{285}$ Ernst Tugendhat, Der Wahrheitsbegriff bei Husserl und Heidegger, S.40. Dazu Hua III/1, S.298.
} 
noetisch-noematisch beschreiben: Auf der noematischen Seite ist der noematische Sinn mit dem Seinscharakter des Wirklichseins verbunden, auf der noetischen Seite ist die noetische Sinngebung im Glaubenscharakter der Glaubensgewißheit vollzogen. Nach dem Prinzip des noetisch-noematischen Parallelismus ist der noetische Glaubenscharakter zuständig für die Konstitution des noematischen Seinscharakters. Husserl appelliert wieder an das Wahrnehmungsbeispiel:

Noetische, auf Seinsmodi korrelativ bezügliche Charaktere - „doxische“ oder „Glaubenscharaktere“ - sind bei den anschaulichen Vorstellungen z.B. der in der normalen Wahrnehmung als „Gewahrung“ reell beschlossene Wahrnehmungsglaube und, des näheren, etwa die Wahrnehmungsgewißheit; ihr entspricht als noematisches Korrelat am erscheinenden „Objekt“ der Seinscharakter, der des ,,wirklich““ ${ }^{286}$

Dasselbe gilt auch für andere objektivierende Akte. Z.B. hat eine sichere Erinnerung noetische Erinnerungsgewißheit und noematischen Seinscharakter des ,wirklich (Gewesen-)Seins“6287, ein sicherer Urteilsakt hat auch noetische Urteilsgewißheit und noematischen Seinscharakter des „wirklich“ usw. ${ }^{288}$ Die noetische Gewißheit ist zuständig für die Konstitution des noematischen Wirklichseins.

(2) In den Logischen Untersuchungen hat Husserl nur den Glaubenscharakter der Gewißheit berührt ${ }^{289}$, in Ideen I führt er aber andere Glaubenscharaktere ein und somit erweitert den Begriffsumfang von Glaubenscharakter. Neben dem wirklich-Seinendes Setzen kann das Subjekt den Gegenstand auch im Modus des „möglich“, des „wahrscheinlich“, des „Zweifelhaft“ usw. setzen. ${ }^{290}$ Husserls Beispiel: Ein wahrgenommener Baum steht zunächst in Gewißheit, plötzlich wird es zweifelhaft, ob wir nicht einer bloßen Illusion zum Opfer gefallen sind. Dabei wird die noetische Sinngebung mit dem Charakter des Zweifelns vollzogen und der noematische Sinn mit dem Seinscharakter des ,zweifelhaft“ verbunden. ${ }^{291}$ Der Glaubenscharakter des

\footnotetext{
${ }^{286}$ Hua III/1: Ideen I, S.239.

${ }^{287}$ Die Zeitkomponente vom „Gewesen“ in der Verbindung von „Gewesen-Sein“ gehört, genauer gesagt, zu dem noematischen Sinn.

${ }^{288}$ Ebd.

${ }^{289} \mathrm{Vgl} .[\S 4]$ der vorliegenden Arbeit.

${ }^{290} \mathrm{Im}$ [§3] der I. LU sind Vermutung und Wahrscheinlichkeitserwägung sehr kurz im Vorbeigehen erwähnt.

291 Seinscharaktere wie „fraglich“, „Zweifelhaft“, genauer gesagt, sind nicht im selben Boot wie die
} 
Zweifelns ist zuständig für die Konstitution des Seinscharakters des ,„Zweifelhaft“ .292

(3) Husserl betont, dass der Glaubenscharakter der Gewißheit und die anderen Glaubenscharaktere nicht zu einer Reihe gleichgeordneter Arten gehören (Hua III/1, S.242). Denn der Glaubenscharakter der Gewißheit als die Urform der Glaubensweise $\mathrm{zu}$ verstehen ist, während andere Glaubenscharakter eher als seine modifizierten Formen oder doxischen Modalitäten gelten. Husserl schreibt:

In der jetzigen Reihe spielt offenbar die Glaubensgewißheit die Rolle der unmodifizierten, oder, wie wir hier $\mathrm{zu}$ sagen hätten, der „unmodalisierten“ Urform der Glaubensweise. Dementsprechend im Korrelat: der $\quad$ Seinscharakter schlechthin (das noematische ,gewiß“ oder ,,wirklich“ seiend) fungiert als die Urform aller Seinsmodalitäten. (Hua III/1, 240)

In diesem Sinne genießen der Glaubenscharakter der Gewißheit und korrelativ der Seinscharakter des Wirklichseins ein begriffliches Privileg gegenüber anderen Glauben- und Seinscharakteren. Deshalb bezeichnet Husserl den Glaubenscharakter der Gewißheit auch als Urglaube oder Urdoxa. (a.a.O., 241)

Im [§106] der Ideen I beschäftigt Husserl sich mit zwei weiteren doxischen Modalitäten, nämlich Verneinung und Bejahung. Diese beiden doxischen Modifikationsformen lassen sich nicht nur auf die Urdoxa, sondern auch auf die oben erwähnten doxischen Modalitäten anwenden. Durch die verneinende Modifikation erfährt ein Glaubenscharakter die „Durchstreichung“ und der entsprechende Seinscharakter das „nicht“ (wirklich vs. nicht-wirklich, möglich vs. nicht-möglich, fraglich vs. nicht-fraglich usw.); die bejahende Modifikation ist keine einfache Wiederholung eines Glaubenscharakters, sondern ,unterstreicht“ ihn, und der Seinscharakter erfährt eine affirmierende Betonung (wirklich vs. wirklich-wirklich, möglich vs. wirklich-möglich, fraglich vs. wirklich-fraglich usw.).(a.a.O., 244)

Außerdem besteht das begriffliche Privileg der Urdoxa auch darin, dass jede

Charaktere „gewiss“, „wirklich“, „möglich“ usw. Im [§21b] von Erfahrung und Urteil diskutiert Husserl das Zweifelsbewußtsein und hält er es für „Schwebezustand“, der ein „Zwiespältigwerden“ oder „Spaltung“ des Bewußtseins ist. Dieser merkwürdige Charakter des Zweifelns oder Für-fraglich-Haltens unterscheidet ,zweifelhaft" und „fraglich“ von anderen Seinscharakteren.

${ }^{292}$ Hua III/1: Ideen I, S.239. 
doxische Modalität eine äquivalente Urdoxa impliziert. Husserl schreibt:

Wir können einerseits, z.B. im Wahrscheinlichkeitsbewußtsein (im Vermuten) lebend, auf das, was wahrscheinlich ist, hinsehen; andererseits aber auf das Wahrscheinliche selbst und als solches, das ist auf das noematische Objekt in dem Charakter, den die Vermutungsnoese ihm zuerteilte. Das „Objekt“ mit seinem Sinnesbestande und mit diesem Wahrscheinlichkeitscharakter ist aber in der zweiten Blickstellung gegeben als seiend: in Beziehung auf dasselbe ist danach das Bewußtsein schlichter Glaube in unmodifiziertem Sinne. Ebenso können wir im Möglichkeitsbewußtsein (in der „Anmutung“), oder im Fragen und Zweifeln leben, den Blick gerichtet auf das, was uns da als möglich, fraglich, zweifelhaft bewußt ist. Wir können aber auch auf die Möglichkeiten, Fraglichkeiten, Zweifelhaftigkeiten als solche hinsehen und ev. explizierend an dem Sinnesobjekt das Möglichsein, Fraglichsein, Zweifelhaftigsein erfassen und prädizieren: es ist dann gegeben als seiend im unmodifizierten Sinne ... den noematischen Charakteren entsprechen prädikable Charaktere an dem Sinnesobjekt, als wirkliche und nicht bloß noematisch modifizierte Prädikabilien. (a.a.O., 242; meine Herv.)

Wir können im negierenden Bewußtsein leben, mit anderen Worten, die Negation ,vollziehen“: der Blick des Ich ist dann gerichtet auf das, was Durchstreichung erfährt. Wir können den Blick aber auch als erfassenden auf das Durchstrichene als solches, auf das mit dem Strich Versehene richten: dann steht dieses als ein neues „Objekt“ da, und zwar da im schlichten doxischen Urmodus „seiend“ ... aber erst in der neuen Einstellung wird der Charakter zur prädikabeln Bestimmung des noematischen Sinneskerns. Ebenso natürlich für die Affirmation. (a.a.O., 244; meine Herv.)

In den Zitaten spricht Husserl von einer Einstellungsänderung, durch die wir von einer doxischen Modalität in eine äquivalente Urdoxa übergehen. Das geschieht, wenn: (i) wir erneut den Glaubenscharakter der Gewißheit übernehmen und (ii) zugleich den von der doxischen Modalität konstituierten Seinscharakter als „,prädikablen Charakter“ in 
den noematischen Kern (= noematischen Sinn) integrieren. Z.B. kann ich durch solche Einstellungsänderung von einer noetischen Sinngebung, die im Glaubenscharakter des ,möglich“ vollzogen wird und deren noematischer Sinn durch „blühender Baum“ ausdrückbar ist, in eine zweite Sinngebung, die im Glaubenscharakter der Gewißheit vollzogen wird und deren noematischer Sinn durch ,,möglicher (blühender Baum)“ ausdrückbar ist, übergehen. Auf der noematischen Seite wird die noematische Einheit [noematischer Sinn (,blühender Baum“) + Seinscharakter (möglich)] in die Einheit [noematischer Sinn (,möglicher (blühender Baum)“) + Seinscharakter (wirklich)] übergeführt. ${ }^{293}$

(4) Die qualitative Modifikation, die einen setzenden objektivierenden Akt in einen entsprechenden nicht-setzenden verwandelt, nennt Husserl in Ideen I Neutralitätsmodifikation. Husserl zufolge ist die Neutralitätsmodifikation wesentlich verschieden von den oben diskutierten Modifikationen. Denn der Output der letzteren ist immer eine Setzung, die den Gegenstand im irgendeinem Seinscharakter setzt. Sogar der Output einer verneinenden Modifikation ist eine negative Setzung, aber keine nichtSetzung. Im Vergleich dazu ist die Neutralitätsmodifikation eine Umwandlung, die alle Setzungskraft aufhebt. Husserl schreibt:

Es handelt sich uns jetzt um eine Modifikation, die jede doxische Modalität, auf die sie bezogen wird, in gewisser Weise völlig aufhebt, völlig entkräftet aber in total anderem Sinne wie die Negation, die zudem, wie wir sahen, im Negat ihre positive Leistung hat, ein Nichtsein, das selbst wieder Sein ist. Sie durchstreicht nicht, sie ,leistet“ nichts, sie ist das bewußtseinsmäßige Gegenstück alles Leistens: dessen Neutralisierung.

Der Setzungscharakter ist kraftlos geworden. Der Glaube ist nun ernstlich kein Glaube mehr, das Vermuten nicht ernstlich Vermuten, das Negieren nicht ernstlich Negieren usw. (a.a.O., 247-248)

Die Neutralitätsmodifikation ist also das „Sich-enthalten“ der betreffenden doxischen Stellungnahme. Den Zitaten ist auch zu entnehmen, dass die Neutralitätsmodifikation

\footnotetext{
${ }^{293}$ Die noematische Einheit des noematischen Sinnes und Seinscharakters nennt Husserl noematischen Satz. Davon später in [§38] und [§46].
} 
nicht nur, wie in den Logischen Untersuchungen, auf die Urdoxa, sondern auch auf andere Glaubenscharaktere anwendbar ist.

Nach dem Prinzip des noetisch-noematischen Parallelismus verändert sich auch der Seinscharakter bei der Neutralitätsmodifikation:

Es ist „,neutralisiertes“ Glauben, Vermuten, Negieren u. dgl., dessen Korrelate diejenigen der unmodifizierten Erlebnisse wiederholen, aber in radikal modifizierter Weise: das Seiend schlechthin, das Möglich-, Wahrscheinlich-, Fraglich-seiend, ebenso das Nicht-seiend und jedes der sonstigen Negate und Affirmate - ist bewußtseinsmäßig da, aber nicht in der Weise des ,,wirklich“, sondern als „bloß Gedachtes“, als „,bloßer Gedanke“. (a.a.O., 248)

Daher gewinnen wir auch eine noetisch-noematische Begriffsbildung im Fall der Neutralitätsmodifikation, nämlich (noetisch-)neutralisierter Glaubenscharakter (noematisch-)neutraler Seinscharakter. Der erstere ist zuständig für die Konstitution des letzteren.

Im Vorbeigehen erwähnt Husserl auch das Annehmen oder Ansetzen. Annehmen oder Ansetzen ist ein Zwischending von Setzung und nicht-Setzung: Einerseits ist das Angenommene allein nicht als wirklich-seiend gesetzt, andererseits ist das Annehmen oder Ansetzen in einer umfassenderen Setzungskontext enthalten und an der setzenden Kraft des umfassenderen Kontexts beteiligt. Beispiel für Annehmen oder Ansetzen ist der Vordersatz eines hypothetischen Satzes (,Wenn sie kommt, werde ich mit ihr sprechen."). ${ }^{294}$ Im Vergleich zum Setzen schlechthin kann man also das Annehmen als hypothetisches Setzen bezeichnen; im Vergleich zum Neutralitätsmodus kann man davon sprechen, ob das Annehmen korrekt oder inkorrekt ist: Man zieht Konsequenzen aus einer Annahme, wenn die Konsequenzen falsch sind, dann sagt man auch, dass die Annahme inkorrekt und somit abzuleugnen ist.

\section{§37*. Exkurs: Setzungscharakter von nicht-objektivierenden Akten}

In Ideen I diskutiert Husserl auch den nicht-doxischen Setzungscharakter von

\footnotetext{
${ }^{294}$ Vgl. Hua III/1: Ideen I, S.249.
} 
nicht-objektivierenden Akten. Da er für das Thema der vorliegenden Arbeit weniger relevant ist, brauchen wir Husserls Lehre von nicht-doxischen Setzungscharakteren nur kurz zu skizzieren.

Husserl zufolge ist der noetische Glaubenscharakter Stellungnahme zu dem Seinscharakter des betreffenden Gegenstandes. In analoger Weise nehmen nichtobjektivierende Akte wie Liebe, Wunsch, Freude auch Stellung zu ihrem Gegenstand:

Machen wir uns zunächst klar, daß Gefallensakte ... ebenso Gemüts- und Willensakte jeder Art eben „Akte“, „,intentionale Erlebnisse“ sind, und daß dazu jeweils die ,intentio“, die „Stellungnahme“ gehört; oder anders ausgedrückt: es sind in einem weitesten, aber wesentlich einheitlichen Sinn „Setzungen“, nur eben nicht doxische. Im Vorbeigehen sagten wir oben ganz korrekt, Aktcharakter überhaupt seien „Thesen“ - Thesen im erweiterten Sinn und nur im besonderen Glaubensthesen oder Modalitäten von solchen. (Hua III/1, 269; meine Herv.)

Daher will Husserl sowohl die doxischen Glaubenscharaktere von objektivierenden Akten als auch die nicht-doxischen Setzungscharaktere von nicht-objektivierenden Akten der Gattung thetischer Charakter (oder Thesis bzw. Setzungscharakter) unterordnen.

Auf der noematischen Seite von nicht-objektivierenden Akten wird auch entsprechender Charakter konstituiert:

Wir werden Gründe finden, den Begriff der Thesis über alle Aktsphären zu erweitern und somit z.B. von Gefallensthesen, Wunschthesen, Willensthesen zu sprechen, mit ihren noematisch Korrelaten „gefällig“, „erwünscht“, ,,praktisch gesollt “ u. dgl. (a.a.O., 260; meine Herv.)

Terminologisch wollen wir das Begriffspaar noetisch-thetischer Charakter noematisch-thetischer Charakter, die allgemein auf objektivierende Akte und nichtobjektivierende Akte anwenbar ist, einführen, um die noetisch-noematische Struktur der „Stellungnahme“ zu kennzeichnen. Wir können im allgemeinen Sinne sagen, dass jeder (nicht-neutrale) Akt mindestens einen noetisch-thetischen Charakter und korrelativ einen noematisch-thetischen Charakter enthält und dass der erstere für die 
Konstitution des letzteren verantwortlich ist. Dabei ist impliziert, dass jeder objektivierende Akt mindestens einen noetischen Glaubenscharakter und korrelativ einen noematischen Seinscharakter enthält und dass der erstere für die Konstitution des letzteren zuständig ist.

Laut Husserl ist in jedem nicht-doxischen Setzungscharakter eine Urdoxa impliziert, wie wir es oben [§37-(3)] auch bei doxischen Modalitäten konstatiert haben. Denn ein nicht-doxischer Setzungscharakter lässt sich, wie es bei doxischen Modalitäten der Fall ist, durch Einstellungsänderung in eine äquivalente Urdoxa verwandeln. Auf noematischer Seite ,wird das ,,gefällig“, „,erwünscht“, ,ge-sollt“ usw. prädikabel; denn in der aktuellen Urglaubenssetzung wird es bewußt als gefällig seiend, erwünscht seiend usw.“(a.a.O.)

\section{§38. Setzende Sinngebung und noematischer Satz}

Nach den oben ausgeführten Konstitutionsanalysen enthält jeder Akt auf noetischer Seite die noetische Sinngebung und den noetisch-thetischen Charakter, ihnen entsprechen auf noematischer Seite der noematische Sinn und der noematischthetische Charakter. Die Einheit [Sinngebung + noetisch-thetischer Charakter] ist eben das, was Husserl in Logischen Untersuchungen als intentionales Wesen (qua reellen Bestandteil des Aktes) bezeichnet, d.i. die Einheit [reelle Aktmaterie + reelle Aktqualität]. In Logischen Untersuchungen spricht Husserl auch von dem intentionalen Wesen als Spezies, was durch die reelle Einheit von [Aktmaterie + Aktqualität] instanziiert wird und zum intentionalen Inhalt des Aktes gehört. Doch in Ideen I will Husserl den intentionalen Inhalt noematisch verstehen (Hua III/1, S.298) und spricht von der Einheit [noematischer Sinn + noematisch-thetischer Charakter], die das intentionale Wesen als Spezies ersetzt. Diese noematische Einheit ist in Ideen I als noematischer Satz bezeichnet. ${ }^{295}$

Der Terminus Satz bezieht sich im normalen Gebrauch auf die Bedeutung von

\footnotetext{
${ }^{295}$ Hua III/1, S.305: , Doch scheint es passender, den Terminus Sinn bloß als jene „Materie“ zu definieren, und dann die Einheit von Sinn und thetischem Charakter als Satz zu bezeichnen.“
} 
propositionalen Ausdrücken (Z.B. „Der Himmerl ist blau.“) . Für Husserl ist die Satzbedeutung eines propositionalen Ausdrucks, unter seinem noematischen Bedeutungsbegriff - davon später ${ }^{296}$ - in einer Lesart ${ }^{297}$ eben die noematische Einheit [noematischer Sinn + noematisch-thetischer Charakter] der entsprechenden Bedeutungsintention, die ihrerseits ein propositional-objektivierender $\mathrm{Akt}^{298}$ ist. Doch Husserl will den Begriff des Satzes noch erweitern, so dass er auch die Einheit [noematischer Sinn + noematisch-thetischer Charakter] einer nominalen Bedeutungsintention als noematischen Satz bezeichnet. Nach Husserls noematischem Bedeutungsbegriff ist der noematische Satz einer nominalen Bedeutungsintention, wie im Fall der propositionalen Bedetungsintention, in einer Lesart eben die Bedeutung, die sie einem sprachlichen Ausdruck verleiht. Daher sind Ausdrucksbedeutungen, ob propositional oder nominal, noematische Sätze von bedeutunggebenden Akten. Aber nicht jeder noematische Sinn ist Ausdrucksbedeutung. Z.B. ist der noematische Satz einer Wahrnehmung keine Ausdrucksbedeutung. ${ }^{299}$ Darüber hinaus besitzen nichtobjektivierende Akte auch noematische Sätze (Gefallensätze, Wunschsätze usw.) ${ }^{300}$. Ausdrucksbedeutungen, und besonders propositionale Bedeutungen, bilden also nur eine Sonderklasse von noematischen Sätzen. Husserl gesteht zu, dass er den Satzbegriff hier in einem seltsamen Sinne verwendet:

Der Begriff des Satzes ist damit freilich außerordentlich und vielleicht befremdlich erweitert, aber doch im Rahmen einer wichtigen Wesenseinheit. Beständig ist ja im Auge zu behalten, daß die Begriffe Sinn und Satz für uns nichts von Ausdruck und begrifflicher Bedeutung enthalten, andererseits aber alle ausdrücklichen Sätze, bzw. Satzbedeutungen unter sich befassen. ${ }^{301}$

Wir werden noch Gelegenheit finden, den Begriff noematischer Satz, insbesondere im Hinblick auf Husserls noematischen Bedeutungsbegriff, näher zu erörtern. Hier

\footnotetext{
${ }^{296}$ Darüber diskutieren wir ausführlich im [\$43].

${ }^{297}$ In einer anderen Lesart ist die Satzbedeutung der noematische Sinn der entsprechenden Bedeutungsintention.

${ }^{298}$ Vgl. [\$4] der vorliegenden Arbeit.

299 Der noematische Sinn einer Wahrnehmung kann sich mit einer entsprechenden Bedeutung, nämlich dem noematischen Sinn der Bedeutungsintention, die die Wahrnehmung erfüllt, decken. Doch in einer solchen Erfüllungseinheit liegt die Bedeutung nicht in der Wahrnehmung, sondern in der Bedeutungsintention.

${ }^{300}$ Hua III/1: Ideen I, S.305.

${ }^{301} \mathrm{Ebd}$.
} 
kommt es uns darauf an, eine entsprechende noetisch-noematische Begriffsbildung festzuhalten. Das noetische Moment, das für die Konstitution des noematischen Satzes zuständig ist, ist eben die Einheit [noetische Sinngebung + noetisch-thetischer Charakter], terminologisch wollen wir sie als setzende Sinngebung bezeichnen. Dabei bekommen wir das Begriffspaar noetisch-setzende Sinngebung - noematischer Satz.

\section{§39. Zusammenfassung des Kapitels}

Wir haben im vorliegenden Kapitel angefangen, im Rahmen der phänomenologischen Reduktion konkrete Analysen $\mathrm{zu}$ betreiben. Der phänomenologisch reduzierte Akt enthält Inhalte, die man innerhalb der Reduktion beschreiben kann. Einerseits ist der Inhalt, der reell im Akte enthalten ist und somit dessen Bestandstück ausmacht. Husserl unterscheidet den reellen Inhalt weiter in noetische und hyletische Inhalte. Und ,,die unvergleichlich wichtigeren und reicheren Analysen liegen auf seiten des Noetischen“302, denn die Noesis ist eben das, was „,das Spezifische der Intentionalität hereinbringt". Andererseits betrachtet Husserl die intentionalen Inhalte, die den Akt reell transzendieren und bei Husserl unter dem Titel Noema zusammengefasst werden. Außerdem benutzt Husserl den operativen Begriff von Konstitution, um den Zusammenhang zwischen Noesis und Noema zu charakterisieren: Die Noesis konstituiert das Noema. Aus diesem Grund sind phänomenologische Analysen innerhalb der Reduktion Konstitutionsanalysen.

Genau gesprochen enthält die Noesis verschiedne Momente (oder Charaktere), die jeweils für die Konstitution von ihren korrelativen noematischen Momenten (oder Charakteren) zuständig sind. Daher haben wir das Prinzip des noetisch-noematischen Parallelismus, dem Husserl bei seinen Konstitutionsanalysen immer folgt, explizit formuliert. Nach diesem Prinzip ergeben Konstitutionsanalysen immer Begriffsbildungen, die das Schema „,noetisches X - noematisches Y“ einhalten. Die Überschriften der Paragraphen zeigen schon Einzelfälle des Schemas: „noetische Sinngebung und noematischer Sinn“ (im [§34]), „,noetischer Repräsentationscharakter und noematischer Anschauungscharakter“ (im [§35]), „Noetischer Aufmerksamkeitscharakter und noematischer Auffälligkeitscharakter“ (im [§36]), „Noetischer Glaubenscharakter und noematischer Seinscharakter“ (im

\footnotetext{
${ }^{302}$ Ebd., S. 196 .
} 
[§37]), „noetisch-setzende Sinngebung und noematischer Satz“ (im [§38]). Die noetisch-noematischen Konstitutionsanalysen, die wir eingangs nur sehr allgemein vorstellen konnten, sind durch solche Begriffsbildungen differenziert und detailiert worden.

Allerdings haben wir die bisherige Betrachtung nur auf die konstitutive Leistung von einzelnen Akten beschränkt: Die Noesis des Aktes konstituiert einen bestimmten noematischen Sinn, der mit einem bestimmten noematischen Anschauungscharakter, einem bestimmten Auffälligkeitscharakter usw. verbunden wird. Doch solche noematischen Momente sind nicht das, was der Akt intendiert, sondern der Akt intendiert durch das durch solche Momente charakterisierte Noema ,ein und denselben Gegenstand“, „,der“ auch in anderen Akten durch andere Noemata intendiert werden kann. Dieser Gegenstand-in-Anführungszeichen, wie schon erwähnt (im [§32d]), ist auch eine noematische Gegebenheit, die wir in den in diesem Kapitel durchgeführten punktuellen Konstitutionsanalysen zwar unvermeidlich, aber nur nebenbei erwähnt haben. Im nächsten Kapitel wollen wir diesen Gegenstand-in-Anführungszeichen in den Vordergrund rücken und die an ihm orientierten dynamischen Konstitutionsanalysen durchführen. 


\section{KAPITEL}

\section{DIE NOETISCH-NOEMATISCHEN ANALYSEN IM RAHMEN DER TRANSZENDENTALEN PHÄNOMENOLOGIE II}

\section{§40. Dynamische Analysen}

Das jeweilige Noema eines einzelnen Aktes lässt sich durch die Angabe des noematischen Sinnes, des Anschauungsscharakters, des Auffälligkeitscharakters usw. spezifizieren. (Z.B. [,,blühender Baum“ + wahrnehmungsmäßig gegeben + primär auffällig + wirklich-seiend]) Wird eines dieser noematischen Momente verändert, dann verändert sich auch das Noema.

Doch ein und derselbe Gegenstand kann durch verschiedene jeweilige Noemata gegeben werden: ich kann denselben Gegenstand durch verschiedene noematische Sinne intendieren ( z.B. „,der Besiegte von Waterloo“ vs. „der Sieger von Jena“); ich kann denselben Baum jetzt in primärer Aufmerksamkeit beobachten, danach nur hintergründlich bemerken (noematisch: verschiedene Auffälligkeitscharaktere); ich kann den Baum wahrnehmungmäßig oder erinnerungsmäßig intendieren (noematisch: verschiedene Anschauungscharaktere) usw. Husserl schreibt:

Dem einen Objekt ordnen wir mannigfaltige Bewußtseinsweisen, Akte, bzw. Aktnoemen zu. Offenbar ist dies nichts zufälliges; keines ist denkbar, ohne daß auch mannigfaltige intentionale Erlebnisse denkbar wären, verknüpft in kontinuierlicher oder in eigentlich synthetischer (polythetischer) Einheit, in denen ,es", das Objekt, als identisches und doch in noematisch verschiedener Weise bewußt ist. ${ }^{303}$

Dieses den jeweiligen Noemata gegenüber identische „Objekt“ will Husserl auch im Rahmen der Konstitutionsanalysen betrachten. Er hält es sogar für das innerste Moment oder den Zentralpunkt des Noema (Hua III/1, S.299). Husserl führt den Begriff des bestimmbaren $X$ ein, um diese neue noematische Dimension zu kennzeichnen: Der Akt konstituiert nicht nur seine jeweilige noematische Einheit [noematischer Sinn + Anschauungscharakter + Auffälligkeitscharakter...], sondern auch ein bestimmbares X (= den Gegenstand-in-Anführungszeichen), das auch in anderen jeweiligen Noemata

\footnotetext{
${ }^{303}$ Hua III/1: Ideen I, S.302.
} 
von anderen Akten vorkommen kann. Etwas metaphorisch gesprochen verhält sich das bestimmbare $\mathrm{X}$ zu den jeweiligen Noemata wie ein grammatisches Subjekt zu den es bestimmenden Prädikaten. Husserl:

Dann scheidet sich evident der identische intentionale „Gegenstand“ von den wechselnden und veränderlichen „Prädikaten“. Es scheidet sich als zentrales noematisches Moment aus: der „Gegenstand“, das „,Objekt“, das „Identische“", das ,bestimmbare Subjekt seiner möglichen Prädikate“ - das pure $\mathrm{X}$ in Abstraktion von allen Prädikaten - und es scheidet sich ab von diesen Prädikaten, oder genauer, von den Prädikatnoemen. (Hua III/1, 302)

Wenn wir Husserls grammatische Metapher fortsetzen, können wir das bestimmbare X (=Gegenstand-in-Anführungszeichen) auch als Subjekt-Noema und seine jeweiligen Noemata als Prädikat-Noemata bezeichnen.

Das bestimmbare $\mathrm{X}$, als eine neue noematische Dimension gegenüber den jeweiligen Noemata von einzelnen Akten, eröffnet für die noetisch-noematische Konstitutionsanalysen eine neue Problematik: Wie ist die Konstitution des bestimmbaren X zu beschreiben? Dafür darf man nicht, wie wir es bisher getan haben, sich ausschließlich nach den Analysen von einzelnen Akten orientieren. Denn das bestimmbare $\mathrm{X}$ ist etwas, was die jeweiligen konstitutiven Leistungen von einzelen Akten überschreitet und als ein kooperatives Produkt von synthetisch verbundenen Akten erwächst. Daher muss die Konstitutionsanalyse des bestimmbaren X unter dem oben schon erwähnten Gesichtspunkt der „funktionellen Probleme“ durchgeführt werden. Zur Erinnerung wiederholen wir das Zitat:

An die Stelle der an den einzelnen Erlebnissen haftenden Analyse und Vergleichung, Deskription und Klassifikation, tritt die Betrachtung der Einzelheiten unter dem ,teleologischen“ Gesichtspunkt ihrer Funktion, „synthetische Einheit“ möglich zu machen ... Sie sucht zu erforschen, wie Selbiges, wie objektive, nicht reell immanente Einheiten jeder Art „,bewußte“, „vermeinte“ sind, wie zur Identität des Vermeinten Bewußtseinsgestaltungen sehr verschiedenen und doch wesensmäßig geforderten Baues gehören, und wie diese Gestaltungen methodisch streng zu beschreiben wären. (a.a.O., 197; meie Herv.)

Unter dem funktionellen oder teleologischen Gesichtspunkt betrachten wir die konstitutiven Leistungen der Akte nicht mehr isoliert, sondern im Rahmen der Gesamtleistung von mannigfaltigen Akten, in denen ein und 
derselbe „Gegenstand“ über eine Reihe von jeweiligen Noemata hinweg konstituiert wird. Wir überlegen, wie die einzelnen Akte in einem solchen Zusammenhang zu der Konstitution des „Gegenstandes“ beitragen. Solche Analysen nennen wir dynamische Konstitutionsanalysen.

In den folgenden Paragraphen des Kapitels wollen wir die Grundideen der dynamischen Konstitutionsanalysen erläutern, und zwar dadurch, dass wir uns nach den Beispielsanalysen von Wahrnehmungen und Urteilsakten orientieren.

\section{§41. Die synthetische Einheit von Wahrnehmungsakten}

\section{a) Das jeweilige Wahrnehmungsnoema als Erscheinung}

In einem Wahrnehmungsakt fasst die Noesis, wie oben erläutert (in [§32] und [§34]), die Empfindungen unter einem bestimmten noematischen Sinn (z.B. ,,blühender Baum“) auf, so dass der Akt nicht bloße Empfindungen erlebt, sondern durch die Empfindungen einen Gegenstand wahrnehmungsmäßig repräsentiert. Wir haben auch erwähnt (im [§35]), dass man einen Gegenstand unter demselben noematischen Sinn, aber in einem verschiedenen Repräsentationscharakter intendieren kann. Z.B. kann ich den Baum auch unter dem noematischen Sinn ,,blühender Baum“, aber nur signitiv, also in einer leeren Bedeutungsintention repräsentieren. ${ }^{304}$

Der Unterschied der beiden Akte, angesichts des Repräsentationscharakters, besteht darin, dass die Wahrnehmung den Gegenstand intuitiv (genauer: perzeptiv), während die leere Bedeutungsintention ihn nur signitiv (oder gar nicht intuitiv) repräsentiert. Daher können die beiden Akte sich zu einer Erfüllungseinheit (vgl. [§5]) zusammenschließen, wobei die Bedeutungsintention als bedeutunggebender Akt und die Wahrnehmung als bedeutungerfüllender Akt fungieren. Angesichts des repräsentierten Gegenstandes enthält die Wahrnehmung auch ein intuitives Element, was an der Bedeutungsintention fehlt und nur in einer Erfüllungseinheit ihr zuwächst. Dieses Element nennt Husserl Fülle: ,„Diese Fülle ist also ... ein positives Bestandstück freilich nur bei den intuitiven Vorstellungen, ein Manko bei den signitiven."305 Die Fülle der Wahrnehmung könnte mehr oder weniger sein, je nachdem, ob sie den Gegenstand mit größerer oder geringerer Vollständigkeit anschaulich repräsentiert

\footnotetext{
${ }^{304}$ Hua XIX/2, S.607: ,ZZu jeder intuitiven Intention gehört - im Sinne idealer Möglichkeit gesprochen - eine sich der Materie nach ihr genau anmessende signitive Intention."

305 Ebd., S.608.
} 
Auf der noematischen Seite ist das jeweilige Noema in einem Modus der Fülle konstituiert. Besonders im Fall der Wahrnehmung nennt Husserl das jeweilige Noema, (unter Betonung des noematischen Sinnes im Modus der Fülle) die Erscheinung ${ }^{306}$. Die noematische Erscheinung entspricht einer der drei Lesarten des äquivoken Terminus (noetischen) Erscheinung, die von Husserl einmal aufgelistet sind. ${ }^{307}$ Gurwitsch bemerkt: „im Zusammenhang mit der Wahrnehmung verwendet Husserl häufig den Audsruck „Erscheinung“; gelegentlich kommt sogar der Terminus „Bild“ vor. Beide Termini sind Synonyme für das Wahrnehmungsnoema.“308 Es ist $\mathrm{zu}$ betonen, dass sich die Erscheinung als Wahrnehmungsnoema $\mathrm{zu}$ dem Gegenstand nicht verhält wie ein vermittelndes Gebilde zu einem verborgenen Ding an sich. Wir nehmen nicht zunächst die Erscheinung wahr, um den Baum zu intendieren, sondern der Baum selber ist unter der Erscheinung wahrgenommen und originär (bzw. leibhaft) gegeben - zwischen der Wahrnehmung und ihrem Gegenstand steht kein Stellvertreter. ${ }^{309}$

Obwohl der Wahrnehmungsgegenstand originär gegeben ist, er kann nur einseitig gegeben sein. Genauer gesprochen sind in jeder jeweiligen Erscheinung nur einige Aspekte des Gegenstandes eigentlich wahrgenommen, während andere Aspekte nur ,mitgemeint“ werden. Z.B. kann ich das vor mir stehende Haus nur von diesem oder jenem Beobachtungspunkt betrachten und deshalb nur seine Vorderseite oder Rückseite oder irgendeine Seite eigentlich wahrnehmen. Husserl:

Worauf wir zunächst achten, ist, daß der Aspekt, die perspektivische Abschattung, in der jeder Raumgegenstand unweigerlich erscheint, ihn immer nur einseitig zur Erscheinung bringt ... Die Rede von diesen und jenen Seiten des Gegenstandes, die zu wirklicher Wahrnehmung kommen, ist unvermeidlich ... Eine äußere Wahrnehmung ist undenkbar, die ihr Wahrgenommenes in ihrem sinnendinglichen Gehalt erschöpfte, ein Wahrnehmungsgegenstand ist undenkbar, der in einer abgeschlossenen Wahrnehmung im strengsten Sinn allseitig, nach der Allheit seiner sinnlich

\footnotetext{
${ }^{306}$ Hua III/1, S.306: „Nehmen wir diesen Sinn voll, mit seiner anschaulichen Fülle, so ergibt sich ein bestimmter und sehr wichtiger Begriff von Erscheinung. " Husserls Erscheinungsbegriff ist vieldeutig. Dieser Begriff bezieht sich in Logischen Untersuchungen oft auf reellen Inhalt des Aktes. Vgl. Hua XIX/1, S.134, 201, 359.

${ }^{307}$ Das sind: (1) das konkrete Erlebnis der Anschauung (manchmal abgesehen von der Aktqualität), (2) der angeschaute Gegenstand und (3) die Empfindung. Die hier einschlägige noematische Erscheinung entspricht eben der unter (1) zugeordneten noetischen Erscheinung. Vgl. Hua XIX/2, S.763.

${ }^{308}$ Aron Gurwitsch, Das Bewußtseinsfeld, übersetzt von Werner D. Fröhlich, Walter de Gruyter, Berlin/New York 1975, S.150.

${ }^{309}$ Hua XIX/1: Logische Untersuchungen, S.436. Dazu auch Hua III/1, §43, 52, 90.
} 
anschaulichen Merkmale gegeben sein könnte. ${ }^{310}$

Husserl zufolge ist also der äußerlich wahrgenommene Gegenstand immer und nur durch jeweilige Erscheinung in einer perspektivischen Abschattung originär gegeben. ${ }^{311}$ Doch keine Einzelwahrnehmung ist selbstgenügsam: sie verweist immer auf andere Wahrnehmungen, in denen derselbe Gegenstand durch andere perspektivische Abschattungen weiter wahrgenommen werden kann. Deshalb spricht Gurwitsch von der „Unzulänglichkeit jeder Einzelwahrnehmung“312. Noematisch gesagt ist jede jeweilige Erscheinung (=Wahrnehmungsnoema) ergänzungsbedürftig und verweist immer auf weitere Erscheinungen. Daher schreibt Gurwitsch: „Aus wesentlichen Gründen ... wird jede Einzelwahrnehmung als Phase oder als in einen einheitlichen Prozess eingeordnet erlebt. “313 Das Verweisen von einer jeweiligen Erscheinung auf die weiteren beschreibt Husserl in einer personifizierenden Weise:

Das Wahrgenommene in seiner Erscheinungsweise ist, was es ist, in jedem Moment des Wahrnehmens, <als> ein System von Verweisen ... und in diesen Verweisen ruft es uns gewissermaßen zu: Es gibt hier noch Weiteres zu sehen, dreh mich doch nach allen Seiten, durchlaufe mich dabei mit dem Blick, tritt näher heran, öffne mich, zerteile mich ... So wirst du mich kennenlernen nach allem, was ich bin. ${ }^{314}$

Genauer gesagt sind die Verweise der jeweiligen Wahrnehmung auf weitere Wahrnehmungen (und korrelativ: die Verweise der jeweiligen noematischen Erscheinung auf weitere Erscheinungen) „Tendenzen, Hinweistendenzen, die zu den nicht gegebenen Erscheinungen forttreiben“315. Deshalb können wir mit Gurwitsch sagen, dass die Einzelwahrnehmung nicht selbstgenügsam ist - sie hat immer die Tendenz, sich an einem Prozess von weiteren Wahrnehmungen desselben Gegenstandes zu beteiligen.

In Bezug auf diese weiteren Wahrnehmungen (und noematisch: die weiteren Erscheinungen) entwickelt Husserl den Horizontbegriff. Der Verweis von meiner

\footnotetext{
${ }^{310}$ Hua XI: Analysen zur passiven Synthesis, hrsg. von Margot Fleischer, Martinus Nijhoff, Den Haag 1966, S.3.

${ }^{311}$ Für Husserl ist auch ein durch innere Wahrnehmung gegebenes Erlebnis niemals vollständig wahrgenommen. Vgl. Hua III/1, Ideen I, S.93-93.

${ }^{312}$ Gurwitsch, Das Bewußtseinsfeld, S.168.

${ }^{313}$ Ebd., S.171.

${ }^{314}$ Hua XI: Analysen zur passiven Synthesis, S.5.

315 Ebd.: „Das Wahrnehmen ist, noetisch gesprochen, ein Gemisch von wirklicher Darstellung, die das Dargestellte in der Weise originaler Darstellung anschaulich macht, und leerem Indizieren, das auf mögliche neue Wahrnehmungen verweist. In noematischer Hinsicht ist das Wahrgenommene derart abschattungsmäßig Gegebenes, daß die jeweilige gegebene $<$ Seite $>$ auf anderes Nichtgegebenes verweist, als nicht gegeben von demselben Gegenstand. Das gilt es zu verstehen.“
} 
Einzelwahrnehmung (und der jeweiligen Erscheinung) auf weitere Wahrnehmungen (und Erscheinungnen) geschieht in einer mehr oder minder bestimmten Weise, je nachdem, wie gut ich den Gegenstand kenne. Wenn ich ein mir vertrautes Haus von der Vorderseite beobachte, dann verweist meine jetzige Wahrnehmung (und Erscheinung) auf weitere Wahrnehmungen angesichts der Rückseite in einer sehr bestimmten Weise: ich antizipiere mit großer Gewißheit, dass ich bei weiteren Wahrnehmungen des Hauses eine rote Rückseite sehen werde; wenn das Haus mir fremd ist, dann kann ich nur in sehr unbestimmter Weise antizipieren, dass ich eine Rückseite von irgendwelcher Farbe sehen werde. Metaphorisch gesagt ist die Einzelwahrnehmung (und noematisch, die jeweilige Erscheinung) von einem mehr oder minder bestimmten „Hof“ (Hua XI, 6) von weiteren Wahrnehmungen (und noematisch, von weiteren Erscheinungen) umgeben. Diesen Hof bezeichnet Husserl als den Horizont der Einzelwahrnehmung (und noematisch, der jeweiligen Erscheinung). Eine thematische Untersuchung des Horizontbegriffs würde den Rahmen der vorliegenden Arbeit sprengen, ${ }^{316}$ hier kommt es uns nur darauf an, mithilfe des Horizontbegriffs die dynamische Konstitutionsanalyse weiter $\mathrm{zu}$ vollziehen. Dafür genügt schon Husserls etwa metaphorische Erörterung (,Hof") des Horizontbegriffs.

\section{b) Enthüllung des Horizonts und der kontinuierliche Wahrnehmungsprozess}

Der Horizont bestimmt auch, so Husserl, Potentialitäten ${ }^{317}$ für das Bewußtsein. Das bedeutet, „man kann jeden Horizont nach dem, was in ihm liegt, befragen, ihn auslegen, die jeweiligen Potentialitäten des Bewußtseinslebens enthüllen."318 Diese Potentialitäten werden verwirklicht und somit wird der Horizont enthüllt, wenn ich, zum Beispiel, um das Haus herumgehend, die vorher unsichtigen Seiten in einer Reihe weiterer Einzelwahrnehmungen beobachte. In diesem kontinuierlichen Wahrnehmungsprozess werden die eigentlich nicht-wahrgenommenen und somit nur im Horizont mitgemeinten Merkmale des Gegenstandes in Modus des ,eigentlichwahrgenommen“ umgewandelt; zugleich werden die eigentlich wahrgenommenen Merkmale in Modus des ,eigentlich nicht-wahrgenommen“ übergeführt und somit in den Horizont des Mitgemeinten gerückt, denn in jeder Phase des kontinuierlichen Wahrnehmungsprozesses ist der Gegenstand nur einseitig originär gegeben. Doch was

\footnotetext{
${ }^{316}$ Mehr darüber vgl. David Woodruff Smith and Ronald McIntyre, Husserl and Intentionality, S.227ff.

${ }^{317}$ Hua I: Cartesianische Meditationen und Pariser Vorträge, S.82.

${ }^{318}$ Ebd.
} 
in den früheren Phasen sichtig war und nun unsichtig wird, ,,ist für unsere Kenntnis nicht verloren“3119, so Husserl:

Dabei verliert sich, was schon vom Gegenstand in die Erscheinung getreten war, partiell wieder im Fortgang aus der Erscheinungsgegebenheit, das Sichtige wird wieder unsichtig. Aber es ist nicht verloren. Es bleibt retentional bewußt und in der Form, daß der Leerhorizont der Erscheinung, die gerade aktuell ist, nun eine neue Vorzeichnung erhält, die bestimmt auf das schon früher gegeben Gewesene als Mitgegenwärtiges verweist. ${ }^{320}$

Das heißt, obwohl die früheren Phasen des Wahrnehmungsprozesses (noematisch: die früheren jeweiligen Erscheinungen) in den Horizont gerückt sind, sie sind noch retentional ${ }^{321}$ beibehalten. Der Horizont erfährt ein Update - die in ihm implizierten Elemente sind uns schon bekannt. Daher können sich die früheren Phasen des Wahrnehmungsprozesses mit den weiteren Phasen zu einer synthetischen Einheit, die selber ein umfassenderer $\mathrm{Akt}^{322}$ ist, zusammenschließen; korrelativ setzen sich die früheren Erscheinungen mit den weiteren auch $\mathrm{zu}$ einer synthetischen Erscheinungseinheit zusammen. ${ }^{323}$ Dabei wird die Einseitigkeit der jeweiligen Erscheinungen ,,relativ überwunden“324, denn in einem solchen Prozess ist immer ein und derselbe Gegenstand in verschiedenen perspektivischen Abschattungen originär gegeben. Husserl schreibt:

Nehme ich z.B. das Wahrnehmen dieses Würfels zum Thema der Beschreibung, so sehe ich ... daß dieser Würfel kontinuierlich als gegenständliche Einheit gegeben ist in einer vielgestaltigen wandelbaren Mannigfaltigkeit betimmt zugehöriger Erscheinungsweisen. Diese sind in

\footnotetext{
${ }^{319}$ Hua XI: Analysen zur passiven Synthesis, S.9.

${ }^{320}$ Ebd., S.12.

${ }^{321}$ „Retention“ bedeutet, dass das Vergangene in der Gegenwart bleibt. Husserls Standard-Beispiel dafür ist MelodieHören. Er erörtert den Retentionsbegriff kurz in EU (\$23b). Für weitere Ausführungen verweist er aber auf seine „Vorlesungen zur Phänomenologie des inneren Zeitbewußtseins“. Das würde ein anderes Thema sein. Hier genügt es, grob zu sagen: Retention heißt, dass die eben vergangenen Bewußtseinsphasen noch in der Bewußtseinsgegenwart bleiben und wirken.

${ }^{322}$ Hua III/1: S.335: „Hat man zunächst ... den inneren Bau der intentionalen Erlebnisse nach allen allgemeinen Strukturen kennengelernt ... so gilt es bei allen synthetischen Einigungen völlig klarzumachen, wie mit ihnen nicht bloß überhaupt Aktverbindungen statthaben, sondern Verbindung zur Einheit eines Aktes.“

${ }^{323}$ Dabei stellt sich die Frage: wie viele Phasen müssen sich ansammeln, damit sie sich zu einer Akteinheit zusammenschließen und korrelativ ein und derselbe Gegenstand sich konstituiert. Meines Erachtens ist eine einzige Phase der äußeren Wahrnehmung schon in der Lage, einen Akt zu bilden, der den Gegenstand in einem sehr uninteressanten und allgemeinen Sinne wie etwas Räumliches intendiert; aber um den Gegenstand in einer interessanten und besonderen Weise (z.B. als ein Haus) kennenzulernen, sollen sich mehr Phasen akkumulieren. Wie viele Phasen man braucht, um den Gegenstand in einer interessanten Weise (z.B. als ein Haus) wahrzunehmen, hängt von den Faktoren wie Vertrautheit des Subjekts mit dem fraglichen Gegenstand oder anderen ähnlichen Gegenständen, Aufmerksamkeit des Subjekts, usw. ab. Die Antwort auf die Frage ist also individuell verschieden. ${ }^{324}$ Hua XIX/2, Logische Untersuchungen, S.599.
} 
ihrem Ablauf nicht ein zusammenhangsloses Nacheinander von Erlebnissen.

Sie verlaufen in der Einheit einer Synthesis, dergemäß in ihnen ein und dasselbe als Erscheinendes bewußt wird. ${ }^{325}$

In diesen überaus komplizierten und wundersamen Systemen der Intention und

Erfüllung, die die Erscheinungen machen, konstituiert sich der immer neu immer anders erscheinende Gegenstand als derselbe. ${ }^{326}$

Wir dürfen hier auch das Prinzip des noetisch-noematischen Parallelismus anwenden und die Sachlage so beschreiben: Auf noetischer Seite wird ein umfassenderer Akt, eine synthetische Einheit von Einzelwahrnemungen vollzogen, auf noematischer Seite wird eine synthetische Einheit von jeweiligen Erscheinungen und somit ein und derselbe Gegenstand konstituiert.

Aufmerksame Leser haben bestimmt bemerkt, dass wir hier bei der dynamischen Konstitutionsanalyse des kontinuierlichen Wahrnehmungsprozesses von der Konstitution ein und desselben Gegenstandes sprechen, ohne die Anführungszeichen hinzuzufügen. Doch oben im [\$40] sprechen wir von der Konstitution ,„ein und desselben Gegenstandes" (in Anführungszeichen), den wir auch als das betimmbare X bezeichnet haben. Der Einfachheit halber machten wir in obigen Ausführungen keine explizite Unterscheidung zwischen den beiden. Aber sind die beiden einerlei?

Im Fall einer veridischen Wahrnehmung scheint es so, dass der Gegenstand-ohneAnführungszeichen, der wirklich in der Welt existiert und wahrgenommen wird, und der Gegenstand-in-Anführungszeichen, der das innerste Moment des Noema ausmacht, zusammenfallen. In seinen Konstitutionsanalysen des Wahrnehmungsprozesses, die wir oben dargestellt haben, hat Husserl tatsächlich keine Unterscheidung zwischen den beiden Gegenstandsbegriffen gemacht. ${ }^{327}$

Die Ansicht der sogenannten East-Coast-Interpretation (Drummond) - davon noch später - lautet: Das bestimmbare X ist identisch mit dem wirklichen Gegenstand. Im Falle einer veridischen Wahrnehmung scheint diese Ansicht im Recht zu sein. Doch die Voraussetzung, dass die Wahrnehmung veridisch ist und daher der Gegenstand wirklich existiert, ist eben eine Voraussetzung, die die phänomenologische Reduktion einklammert. Der Phänomenologe muss auch, wenn er innerhalb der Reduktion

\footnotetext{
${ }^{325}$ Hua I: Cartesianische Meditationen und Pariser Vorträge, S.78.

${ }^{326}$ Hua XI: Analysen zur passiven Synthesis, S.13.

${ }^{327}$ Z.B.: „In jedem Moment ist der gegenständliche Sinn derselbe hinsichtlich des Gegenstandes schlechthin, der gemeinter ist, und ist in der kontinuierlichen Abfolge der Momentanerscheinungen in Deckung. So etwa dieser Tisch da. Aber dieses Identische ist ein beständiges x, ist ein beständiges Substrat von wirklichen erscheinenden TischMomenten, aber auch von Hinweisen auf noch nicht erscheinende." Ebd., S.5. Vgl. auch Hua III/1, S.331,347, 349.
} 
arbeiten will, die Möglichkeit, dass die Wahrnehmung nicht veridisch ist, erlauben und berücksichtigen. ${ }^{328}$ Denn phänomenologisch wird ein bestimmbares $\mathrm{X} \quad \mathrm{im}$ Wahrnehmungsprozess auch dann konstituiert, wenn die Wahrnehmung tatsächlich Halluzination ist und es keinen Gegenstand-ohne-Anführungszeichen gibt, der da wahrgenommen wird. ${ }^{329}$ Wie können die beiden zusammenfallen, wenn eines davon nicht existiert? Wie verhalten sich die beiden Gegenstandsbegriffe im Rahmen der Reduktion zueinander? Um solche Fragen zu beantworten, möchte ich Husserls Begriff des bestimmbaren $\mathrm{X}$ (=Gegenstand-in-Anführungszeichen) nochmals betrachten und nötigenfalls kritisch modifizieren.

\section{§42. Das bestimmbare $\mathrm{X}$ und der Gegenstand der Wahrnehmung}

\section{a) Zwei Begriffe des bestimmbaren $X$}

In seinem Aufsatz Husserls Begriff des Noema vertritt Rudolf Bernet die Ansicht, dass Husserls Begriff des Noema schon vor Ideen $I$ in zwei verschiedenen Zusammenhängen verwendet wurde - „einerseits im Zusammenhang einer phänomenologischen Erkenntnistheorie und andererseits im Zusammenhang der Ausbildung einer phänomenologischen „Bedeutungslehre“ “330. In den beiden verschiedenen Zusammenhängen wird das Noema und dessen Beziehung auf den Gegenstand verschieden bestimmt. Bernet glaubt, diese verschiedenen Bestimmungen des Noema liefen in Ideen I durcheinander und verursachten daher Kontroversen zwischen Husserls Interpreten. Dasselbe gilt auch für das innerste Moment des Noema, nämlich das bestimmbare $\mathrm{X}$. Genau gesprochen hat Bernet zwei X-Begriffe unterschieden, die wir im Folgenden jeweils als X-Begriff 1 und X-Begriff 2 bezeichnen.

Die Unterscheidung zwischen den beiden lässt sich durch Husserls Text zusammenfassen:

„Gegenstand“ ist für uns überall ein Titel für Wesenszusammenhänge des Bewußtseins; er tritt zunächst auf als noematisches X, als Sinnessubjekt verschiedener Wesenstypen von Sinnen und Sätzen. Er tritt ferner auf als Titel „,wirklicher Gegenstand“ und ist dann Titel für gewisse eidetisch betrachtete Vernunftzusammhänge, in denen das in ihnen sinngemäß

\footnotetext{
${ }^{328} \mathrm{Vgl}$. unten [\$42c] der vorliegenden Arbeit.

${ }^{329} \mathrm{Vgl}$. Hua III/1: Ideen I, S.207.

${ }^{330}$ Rudolf Bernet, „Husserls Begriff des Noema“, S.62.
} 
einheitliche $X$ seine vernunftmäßige Setzung erhält. ${ }^{331}$

(1) X-Begriff 1 : Bernet zufolge hat Husserl in dem bedeutungstheoretischen Zusammenhang, wo er sich mit einer Bestimmung der gegenständlichen Beziehung der Bedeutung beschäftigt, ohne zu berücksichtigen, ob der betreffende Gegenstand wirklich existiert, den Begriff ${ }_{1}$ von Gegenstand-in-Anführungszeichen bzw. $X$ entwickelt. Unter diesem X-Begriff ${ }_{1}$ versteht man das konstituierte $\mathrm{X}$, das in einem Akt oder Aktzusammenhang konstituiert wird, ohne $\mathrm{zu}$ fragen, ob das $\mathrm{X}$ mit einem wirklichen Gegenstand zusammenfällt. Der Bedeutung eines falschen Urteilsaktes (z.B. „Napoleon entdeckt Amerika“) entspricht kein wirklicher Gegenstand (Sachverhalt), aber das hindert nicht, dass auch in diesem Akt ein Gegenstand-inAnführungszeichen bzw. X, als Moment der jeweiligen Urteilsbedeutung und Einheitspunkt von mannigfaltigen Urteilsbedeutungen ${ }^{332}$, konstituiert wird. Unter diesem X-Begriff, dürfen wir mit Bernet sagen, „,Die Gegebenheit des X als Moment des ideal-identischen Sinnes präjudiziert nichts über die Wahrheit dieses Sinnes bzw. die Wirklichkeit eines ihm „entsprechenden“ Sachverhalts.“333

(2) X-Begriff 2 : Anders verhält es sich in dem sogenannten erkenntnistheoretischen Zusammenhang, wo es in Frage kommt, ob der X-Konstitution ein wirklicher Gegenstand entspricht. In Ideen $I$ diskutiert Husserl diese Frage in dem Abschnitt „Vernunft und Wirklichkeit“. Unter X-Begriff 2 verstehen wir das bestimmbare X, dessen noematischer Seinscharakter des Wirklichseins durch einen vernünftig motivierten Akt oder Aktzusammenhang unterstützt wird. Innerhalb der phänomenologisch-erkenntnistheoretischen Betrachtung fasst Husserl solche vernünftig motivierten Akte oder Aktzusammenhänge unter dem Titel Vernunftbewußtsein zusammen. ${ }^{334}$ Ein ausgezeichneter Fall von Vernunftbewußtsein ist eben der Wahrnehmungsprozess, den wir in letzten Paragraphen betrachtet haben und im Folgenden nochmals, besonders im Hinblick auf das Verhältnis zwischen X und wirklichem Gegenstand, betrachten wollen.

Die Einzelwahrnehmungen schließen sich, wie oben schon beschrieben, im Wahrnehmungsprozess zu einer synthetischen Einheit zusammen; auf noematischer

\footnotetext{
${ }^{331}$ Hua III/1: Ideen I, S.356.

${ }^{332}$ Wenn ich zugleich auch urteile, dass der Sieger von Jena Amerika entdeckt, mit der Erkenntnis, dass Napoleon der Sieger von Jena ist, dann beziehe ich mich auf denselben X-Sachverhalt durch verschiedene Bedeutungen. Dabei spielt derselbe X-Sachverhalt als der Einheitspunkt von verschiedenen Bedeutungen (,Napoleon entdeckt Amerika“, „Der Sieger von Jena entdeckt Amerika“" usw.).

${ }^{333}$ Rudolf Bernet, „Husserls Begriff des Noema“, S.77.

${ }^{334}$ Hua III/1: Ideen I, S.314, 322.
} 
Seite konstituiert sich dabei ein bestimmbares X. Husserl zufolge wird der noetische Setzungscharakter der Glaubensgewißheit in Bezug auf das $\mathrm{X}$ durch die wahrnehmungsmäßigen Erscheinungen (besonders im Blick auf ihre noematischen Sinne im Anschauungscharakter des „originär“ oder „leibhaft“) „vernünftig motiviert“ (Hua III/1, S.316). Das verwundert nicht, denn ein ausgezeichneter Rechtsgrund (a.a.O.) für die Glaubensgewißheit in Bezug auf einen Gegenstand liegt darin, dass der Gegenstand im Modus des „originär“ gegeben wird. Das findet eben in wahrnehmungsmäßigen Erscheinungen statt. Mit Husserls eigenen Worten, „die Setzung hat in der originären Gegebenheit ihren ursprünglichen Rechtsgrund“"(a.a.O.) oder „vernünftig motiviert ist die Setzung dabei nur durch die Erscheinung“(a.a.O., 319). Dabei gewinnt der Setzungscharakter (Glaubensgewißheit) den Vernunftcharakter: Er ist vernünftiger Setzungscharakter oder Vernunftsetzung. Auf noematischer Seite fungiert die Erscheinung (besonders mit Blick auf ihren Sinn im Anschauungscharakter des „originär“ oder „leibhaft“) als Unterlage für den Seinscharakter des Wirklichseins in Bezug auf das dabei konstituierte X. ${ }^{335}$

In einem glatt verlaufenden Wahrnehmungsprozess, wo die Erscheinungen sich miteinander einstimmig zusammenschließen, wird die Glaubensgewißheit nicht nur vernünftig motiviert, sondern im Laufe des Prozesses immer mehr verstärkt - „sie gewinnt beständig an „Gewicht“ “(a.a.O., 320). Deshalb spricht Husserl von ,eine(r) positive(n) Steigerung in Hinsicht auf ihre motivierende „Kraft“ “( (a.a.O.). Korrelativ wird in Bezug auf die noematische Seite der noematische Seinscharakter des Wirklichseins im Verlauf der einstimmigen Erscheinungen immer mehr bestätigt.

Obwohl die Vernunftsetzung in Bezug auf das X im Wahrnehmungprozess immer verstärkt und das $\mathrm{X}$ in jeder geschlossen Phase des Prozesses mehrseitig konstituiert wird, das X kann niemals allseitig (adäquat) konstituiert werden. Daher kann keine in einem abgeschlossenen Wahrnehmugnsprozess vollzogene Vernunftsetzung endgültig oder unüberwindlich sein; auf noematischer Seite ist der Seinscharakter des

\footnotetext{
${ }^{335}$ Ebd., S.315: „In der Einstellung auf das Noema finden wir den Charakter der Leibhaftigkeit (als originäre Erfülltheit) mit dem puren Sinne verschmolzen, und der Sinn mit diesem Charakter fungiert nun als Unterlage des noematischen Setzungscharakters, oder was hier dasselbe sagt: des Seinscharakters. Das Parallele gilt in der Einstellung auf die Noese."
} 
Wirklichseins in Bezug auf das X nicht endgültig. Z.B. kann der Vorgang einer Halluzination, unter Erster-Person-Perspektive betrachtet, genauso einstimmig verlaufen, wie es bei einer veridischen Wahrnehmung der Fall ist. Dabei wird auch ein bestimmbares $\mathrm{X}$ vernünftig konstituiert, und zwar im Charakter des Wirklichseins, aber in diesem Fall gibt es gar keinen wirklichen Gegenstand, mit dem das $\mathrm{X}$ zusammenfallen kann. Die vernünftige X-Konstitution garantiert also nicht, dass der Wahrnehmungsprozess sich auf einen wirklichen Gegenstand bezieht (oder dass, gilt: $\mathrm{X}=$ wirklicher Gegenstand).

Um das Zusammenfallen zwischen X und wirklichem Gegenstand herzustellen, müssen wir die Voraussetzung hinzufügen: Der Wahrnehmungsprozess ist veridisch; anders gewendet, der Gegenstand, der so ist, wie das konstituierte $\mathrm{X}$ in Konstitutionsanalyse beschrieben ist, existiert wirklich. Husserl macht diese Voraussetzung mit Hinweis auf den weiteren Fortgang des Wahrnehmugnsprozesses:

Prinzipiell kann ein Dingreales, ein Sein solchen Sinnes in einer abgeschlossenen Erscheinung nur, ,inadäquat“" erscheinen. Damit hängt wesenmäßig zusammen, daß keine auf solch einer inadäquat gebenden Erscheinung beruhende Vernunftsetzung „endgültig“, keine „unüberwindlich“ sein kann; daß keine in ihrer Vereinzelung gleichwertig ist mit dem schlechthinnigen: „Das Ding ist wirklich“, sondern nur gleichwertig ist mit dem: „Es ist wirklich“ - vorausgesetzt, daß der

\section{Fortgang der Erfahrung nicht „stärkere Vernunftmotive“ herbeibringt,} welche die ursprüngliche Setzung als eine in dem weiteren Zusammenhang ,durchzustreichende“ herausstellen. ${ }^{336}$ (meine Herv.)

Mit dem Hinweis auf den weiteren Wahrnehmungsfortgang, der das bisher konstituierte $\mathrm{X}$ nicht durchstreicht, d.h. als etwas Illusionäres oder Halluziniertes herausstellt, sondern weiter bestätigt, glaubt Husserl, sicherstellen zu können, dass der bisherige Wahrnehmungsprozess veridisch ist. ${ }^{337}$ Unter der Voraussetzung, dass die

\footnotetext{
${ }^{336}$ Ebd., S.319.

${ }^{337}$ Aber wieweit muss der einstimmige Vorgang weiter verlaufen, um dies zu versichern? Husserls Antwort: unendlich weit. Denn in jeder abgeschlossen Phase des weiteren Vorgangs ist das X nur in endlich vielen Erscheinungen inadäquat (nicht-allseitig) gegeben; dabei wird immer offen gelassen, ob der noch weitere Vorgang das X „durchstreicht“. Husserl konzipiert eine unendliche Fortsetzung des Wahrnehmungsprozesses, in der das X
} 
Wahrnehmung veridisch ist, können wir mit Recht die Disquotation vollziehen: der Gegenstand-in-Anführungszeichen (das $\mathrm{X})=$ der Gegenstand-ohneAnführungszeichen, oder, „,der Gegenstand“ = der Gegenstand.

(3) Eigentlich schließen sich die beiden X-Begriffe nicht gegenseitig aus. Ein im Vernunftbewußtsein konstituiertes $\mathrm{X}(\mathrm{X} \text { unter Begriff })_{2}$ ist ein Sonderfall von $\mathrm{X}$ überhaupt, ob es sich vernünftig konstituiert oder nicht (X unter Begriff 1 ). Daher ist XBegriff $_{1}$ ein Oberbegriff $\mathrm{zu}$ X-Begriff 2 . Die beiden kennzeichnen verschiedene Arbeitsstufen von Konstitutionsanalysen des bestimmbaren X:

Die Frage ist also, wie in phänomenologischer Wissenschaftlichkeit all die Bewußtseinszusammennhänge noetisch, bzw. noematisch zu beschreiben sind, die einen Gegenstand schlechthin (was im Sinne der gewöhnlichen Rede immer einen wirklichen Gegenstand besagt), eben in seiner Wirklichkeit notwendig machen. In weiteren Sinne aber ,konstituiert“ sich ein Gegenstand - ,ob wirklicher ist oder nicht“ - in gewissen Bewußtseinszusammenhängen, die in sich eine einsehbare Einheit tragen, sofern sie wesenmäßig das Bewußtsein eines identischen X mit sich führen. ${ }^{338}$

„Gegenstand“ ist für uns überall ein Titel für Wesenszusammenhänge des Bewußtseins; er tritt zunächst auf als noematisches X, als Sinnessubjekt verschiedener Wesenstypen von Sinnen und Sätzen. Er tritt ferner auf als Titel „,wirklicher Gegenstand“ und ist dann Titel für gewisse eidetisch betrachtete Vernunftzusammhänge, in denen das in ihnen sinngemäß einheitliche X seine vernunftmäßige Setzung erhält. ${ }^{339}$

Wir können sagen: Die Untersuchung des bestimmbaren X im Sinne vom Begriff 2 ist eine besondere Branche der allgemeineren Untersuchung des bestimmbaren X im Sinne vom Begriff 1 , genau wie die Theorie von Primzahl eine Teildisziplin der allgemeinen Zahlentheorie bildet.

immer vollständiger gegeben wird und sich im Unendlichen der adäquaten Gegebenheit annähert. Solche unendliche Fortsetzung ist praktisch unerreichbar und kennzeichnet für Husserl eine kantische Idee. Husserl glaubt, in einem solchen durch kantische Idee konzipierten Wahrnehmungsprozess wird der Gegenstand adäquat und originär gegeben, und die adäquat-originäre Gegebenheit garantiert, dass der Gegenstand wirklich existiert. Vgl. Hua III/1, $\S 142-143$.

${ }^{338}$ Hua III/ $/ 1$, S. 332 .

${ }^{339}$ Ebd., S.356. 


\section{b) Das bestimmbare $X$ als wirklicher Gegenstand}

Man könnte bezweifeln, ob es für Husserl möglich ist, dass das $\mathrm{X}$ als ein noematisches Moment zugleich ein wirklicher Gegenstand ist. Denn zwei berühmte, vielleicht berüchtigte Zitate von Husserl lauten:

Der Baum schlechthin, das Ding in der Natur, ist nichts weniger als dieses Baumwahrgenommene als solches, das als Wahrnehmungssinn zur Wahrnehmung und unabtrennbar gehört. Der Baum schlechthin kann abbrennen, sich in seine chemischen Elemente auflösen usw. Der Sinn aber Sinn dieser Wahrnehmung, ein notwendig zu ihrem Wesen Gehöriges - kann nicht abbrennen, er hat keine chemischen Elemente, keine Kräfte, keine realen Eigenschaften. ${ }^{340}$

Von einem Baum schlechthin kann ausgesagt werden, er verbrenne, ein wahrgenommener Baum ,als solcher“ kann nicht verbrennen; nämlich von ihm das aussagen, ist widersinnig; denn dann mutet man einer Komponente einer reinen Wahrnehmung, die nur als eigenwesentliches Moment eines Ichsubjekts denkbar ist, zu, etwas zu tun, was für einen Körper aus Holz Sinn haben kann: zu verbrennen. ${ }^{341}$

Man könnte also einwenden, dass nach diesen Zitaten das Baum-Noema kein physischer Gegenstand sein kann, während der wirkliche Baum doch etwas Physisches ist. Zwischen Noema und Gegenstand besteht dann ein ontologischer Unterschied. Wegen dieses ontologischen Unterschiedes soll das X, als ein Moment des Noema, niemals mit dem wirklichen Gegenstand (Baum) zusammenfallen.

Darauf würde Bernet antworten: Der Terminus Noema in den Zitaten bezieht sich auf das jeweilige noematische Korrelat (= die jeweilige Erscheinung, in unseren Worten) der Warhnehmung, dem keine real-physikalischen Eigenschaften zukommen; davon muss man den in diesen jeweiligen Noemata konstituierten Gegenstand-inAnführungszeichen (= das X) unterscheiden, und dieser konstituierte Gegenstand-in-

\footnotetext{
${ }^{340}$ Ebd., S.205.

${ }^{341}$ Hua VI: Die Krisis der europäischen Wissenschaften und die transzendentale Phänomenologie, hrsg. von Walter Biemel, Martinus Nijhoff, Den Haag 1954, S.245.
} 
Anführungszeichen $(=$ das $\mathrm{X})$ ist ontologisch ein physischer Gegenstand. ${ }^{342}$ Er schreibt:

Das konstituierte Ding ist zwar eine Form der noematischen Gegebenheit, aber dennoch Gegebenheit eines räumlichen, transzendenten Naturgegenstandes ... Leider unterlässt es Husserl in beiden Passagen[= den obigen Zitaten], ausdrücklich darauf hinzuweisen, dass das als konstituierter Gegenstand gefasste Noema wohl abbrennen kann und dass die Möglichkeit seines Abbrennens als phänomenologisch konstituierte real-kausale Eigenschaft des Dinges verstanden werden muss. ${ }^{343}$

Bernets Ansicht enthält zwei Punkte: (1) Man muss zwischen dem Noema als jeweiliger Erscheinung und dem als in mannigfaltigen Erscheinungen konstituierten Gegenstand-in-Anführungszeichen(=X) unterscheiden; (2) Das Wahrnehmungsnoema als konstituierter Gegenstand-in-Anführungszeichen $(=\mathrm{X})$ ist wirklicher Gegenstand. Punkt (1) stimme ich voll und ganz zu, er entspricht tatsächlich unserer Unterscheidung zwischen der punktuellen Konstitutionsanalyse, die die konstitutive Leistung von Einzelakten (das jeweilige Noema) untersucht, und der dynamischen Konstitutionsanalyse, die die synthetisch-konstitutive Leistung von Aktzusammenhang (insbesondere das X) studiert.

Punkt (2) kann ich nur vorbehaltlich zustimmen und ich möchte ihn kritisch rekonstruieren. Zwar ist zuzugeben, dass das als konstituierter Gegenstand erfasste Wahrnehmungsnoema ein bestimmbares $\mathrm{X}$ im Sinne vom X-Begriff ${ }_{2}$ ist und deshalb im vernünftig motivierten Seinscharakter des Wirklichseins konstituiert wird - es ist vernünftig, dieses $\mathrm{X}$ für einen wirklichen Gegenstand zu halten. Allerdings kann es passieren, dass der Fortgang des Wahrnehmungsprozesses das bisherige $\mathrm{X}$ durchstreicht. Deshalb darf man das bestimmbare X im Sinne vom X-Begriff 2 nicht ohne weiteres als wirklichen Gegenstand betrachten. Man muss, wie oben schon erwähnt, die Voraussetzung, dass der bisherige Wahrnehmungsprozess veridisch ist, hinzufügen, um das X für einen wirklichen Gegenstand halten zu können. In seinen Ausführungen hat Bernet unterlassen, diese Voraussetzung explizit zu machen, daher

\footnotetext{
${ }^{342}$ Rudolf Bernet, „Husserls Begriff des Noema“, S.67, 70.

${ }^{343}$ Ebd., S.70.
} 
hat er das $\mathrm{X}$ der Wahrnehmung unmitterlbar als einen wirklichen, physischen Gegenstand angesehen. Ohne diese Voraussetzung würde Bernets stark idealistische Interpretation des $\mathrm{X}$ zu der absurden Möglichkeit führen, dass ein wirklicher Gegenstand (wie ein Baum) durch den weiteren Fortgang des Wahrnehmungsprozesses vernichtet wird, weil der weitere Fortgang der Wahrnehmung die bisherige Vernünftigkeit der X-Konstitution „,durchstreichen“ kann. ${ }^{344}$

Wir können Bernets Antwort auf den obigen Einwand wie folgt rekonstruieren: Der Terminus Noema in den Zitaten bezieht sich auf das jeweilige noematische Korrelat (= die jeweilige Erscheinung, in unseren Worten) der Warhnehmung, dem keine realphysikalischen Eigenschaften zukommen; davon muss man den in diesen jeweiligen Noemen konstituierten Gegenstand-in-Anführungszeichen (= das X) unterscheiden, und dieser konstituierte Gegenstand-in-Anführugnszeichen (= das X) ist ontologisch ein physischer Gegenstand, vorausgesetzt, dass der Wahrnehmungsprozess veridisch ist. Daher besteht kein ontologischer Unterschied zwischen dem physichen Gegenstand und dem Noema (im Sinne von X), wenn die Wahrnehmung veridisch ist.

\section{c) Existenzneutrale Spezifikation des bestimmbaren $\mathbf{X}$}

Die Voraussetzung, dass die Wahrnehmung veridisch ist und daher der wahrgenommene Gegenstand wirklich existiert, ist eben etwas, was die

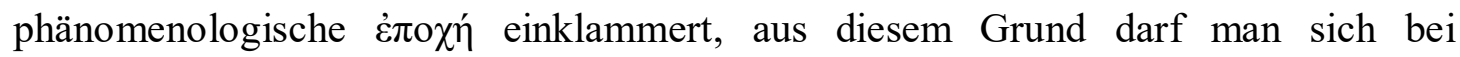
Konstitutionsanalyse des X nicht ausschließlich an dieser Voraussetzung orientieren und einfach behaupten, dass das X der Wahrnehmung ein wirklicher und physischer Gegenstand ist. Beide Fälle sind möglich: (1) Die Wahrnehmung ist veridisch und der Gegenstand, sowie er wahrgenommen wird, wirklich existiert; (2) Die Wahrnehmung ist nichtveridisch (Halluzination oder Illusion ${ }^{345}$ ) und der Gegenstand, sowie er

\footnotetext{
${ }^{344}$ Bei „Explosion“ in Illusionsfällen kann das Noema revidiert werden: z.B. wird ein Baum-Noema durch ein Mensch-Noema ersetzt. Nach Bernets Interpretation würde die Illusion einen Baum schaffen, wenn sie beginnt, und ihn vernichtet oder in einen Mensch umwandelt, wenn sie endet - eine absurde Konsequenz. Ähnliche Konsequenzen gibt es auch in Halluzinationsfällen.

${ }^{345}$ Bei Illusion ist die Situation etwas komplizierter. Denn Illusion (im Gegensatz zu Halluzination) involviert sowohl veridische als auch nicht-veridische Faktoren, z.B. sieht man bei der Müller-Lyer-Illusion zwei wirkliche Linien (veridisch), aber von verschiedenen Längen (nicht-veridisch). Die Frage, ob die illusionäre Wahrnehmung veridisch ist, scheint mir davon abzuhängen, welche Faktoren, die veridischen oder die nicht-veridischen, für die Diskussion maßgeblich sind, was wiederum mit Kontexten variiert, denn in manchen Kontexten interessiert man sich (aus guten Gründen) mehr für die veridischen Faktoren und die nicht-veridischen sind irrelevant, in anderen Kontexten aber umgekehrt. Damit im Zusammenhang hat Smith bei Illusionsfällen verschiedene Stufen des
} 
wahrgenommen wird, existiert nicht in der Wirklichkeit. Eine Spezifikation angesichts des Verhältnisses von X und Gegenstand innerhalb der Reduktion darf keine der beiden Möglichkeiten voraussetzen, sollte jedoch beide Möglichkeiten erlauben, d.h. sie muss in Bezug auf den Gegenstand existenzneutral sein.

Die gewünschte Spezifikation muss also die beiden Forderungen erfüllen:

(1) Sie muss in Bezug auf den Gegenstand existenzneutral sein;

(2) Wegen (1) erlaubt und berücksichtigt sie die beiden Möglichkeiten: entweder das X mit dem wirklichen Gegenstand zusammenfällt, oder nicht.

1894 hat Husserl in seinem Aufsatz ,intentionale Gegenstände“346 überlegt, wie man den Satz (V) ,Jede Vorstellung ${ }^{347}$ hat einen Gegenstand“ als einen wahren Satz interpretieren soll, wenn man mit „Gegenstand“ wirklicher Gegenstand meint. Die Schwierigkeit für eine solche Interpretation besteht darin, dass es Vorstellungen (wie „Pegasus“) gibt, denen kein wirklicher Gegenstand entspricht. Husserl zufolge wird der Satz (V) regiert von einem unausgesprochenen und $\mathrm{zu}$ ergänzenden Assumptionsoperator, den man in der Regel weglässt. ${ }^{348}$ Genau reformuliert soll der Satz lauten: „(i) Jede Vorstellung hat einen Gegenstand oder, (ii) (wenn die Vorstellung nicht veridisch ist) sie würde einen Gegenstand haben, wenn sie veridisch wäre. “349 Durch den disjunktiven Teil (ii), dessen konjunktivische Form hier nach einer alltäglichen Lesart ${ }^{350}$ verstanden wird, kann diese Reformulierung des Satzes (V) den nicht-veridischen Fall berücksichtigen.

In Anlehnung daran schlage ich vor, das bestimmbare $\mathrm{X}$ des Wahrnehmungsprozesses, besonders angesichts des Verhältnisses von $\mathrm{X}$ und Gegenstand, wie folgt zu spezifizieren:

\section{(Spezifikation des bestimmbaren X der Wahrnehmung)}

In jeder Wahrnehmung ${ }^{351}$ wird ein $X$ konstituiert, das entweder

(i) mit dem wirklichen Gegenstand, der so ist, wie ${ }^{352}$ er in der Wahrnehmung

bestimmbaren X differenziert, vgl. David Woodruff Smith, ,,The Case of the Exploding Perception“, in: Synthese 41, S.239-269 (1979).

346 Hua XXII: Aufsätze und Rezensionen (1890-1910), hrsg. von Bernhard Rang, Martinus Nijhoff, The Hague/Boston/London 1979, S.303ff.

${ }^{347}$ Hier bezieht man sich mit ,,Vorstellung“ auf intentionale Akte.

${ }^{348}$ Vgl. Christian Beyer, „Fiktionale Rede“.

${ }^{349}$ Vgl. Chrisitian Beyer, „Noematic Sinn. Gerneral meaning-function or propositional content?““, in: Meaning and Language: Phenomenological Perspectives, S.85.

${ }^{350}$ Die alltägliche Lesart von „Wäre p der Fall, dann würde q der Fall sein“ impliziert, dass p tatsächlich nicht der Fall ist.

${ }^{351}$ Oder in jedem Prozess von Wahrnehmungen, die denselben Gegenstand intendieren oder mindestens so prätendieren.

${ }^{352}$ Was ist hier mit dem „,so ... wie ...“ gemeint? Meines Erachtens ist die Antwort kontextabhängig. Man sieht z.B. bei der Müller-Lyer-Illusion zwei wirkliche Linien (veridisch), aber von verschiedenen Längen (nicht-veridisch). 
intendiert ist, zusammenfällt, oder

(ii) es verhielte sich so, wie in (i) beschrieben, wenn die Wahrnehmung veridisch wäre.

Diese Spezifikation erfüllt beide der oben aufgestellten Forderungen. Zur Forderung (1) hat die Spezifikation weder die einzuklammernde Feststellung, dass die Wahrnehmung veridisch ist und somit der wahrgenommene Gegenstand wirklich existiert, noch die, dass das nicht der Fall ist, vorausgesetzt. Zur Forderung (2) erlaubt sie einerseits durch den disjunktiven Teil (i) die Möglichkeit, dass X der wirklicher Gegenstand ist, andererseits erlaubt sie durch den disjunktiven Teil (ii) die Möglichkeit, dass X kein wirklicher Gegenstand ist.

Nach dieser Spezifikation variiert der noematische Inhalt der Wahrnehmung, je nachdem, ob das $\mathrm{X}$ als noematisches Moment mit dem wirklichen Gegenstand zusammenfällt oder nicht. Ich verstehe das $X$ als eine Art Leerstelle, die in verschiedenen Fällen von verschiedenen Entitäten besetzt wird. Im veridischen Fall ist sie besetzt von dem wirklichen Gegenstand (z.B. ein Baum). ${ }^{353}$ Ich lasse es offen, was ihn im nichtveridischen Fall besetzen wird - Kandidaten könnten psychologische Entität wie ein inneres Bild oder ein nicht-existierender Gegenstand im meinongianischen Sinne usw. sein ${ }^{354}$, oder sie ist von gar nichts besetzt und bleibt leer - welche Möglichkeit man dafür wählt, hängt von seiner Intentionalitäts- und Gegenstandstheorie ab. ${ }^{355}$

Auf diese Weise bekommen wir, besonders im Hinblick auf das Verhältnis von X

Die Frage, ob ein Gegenstand so ist, wie er in der Wahrnehmung intendiert ist, scheint mir davon abzuhängen, welche Faktoren, die veridischen oder die nicht-veridischen, für die Diskussion maßgeblich sind, was wiederum mit Kontexten variiert, denn in manchen Kontexten interessiert man sich (aus guten Gründen) mehr für die veridischen Faktoren und die nicht-veridischen sind irrelevant, in anderen Kontexten aber umgekehrt. Im ersteren Fall sind die zwei Linien eben so, wie sie in der Wahrnehmung intendiert werden, und die Wahrnehmung ist veridisch; im letzteren Fall sind sie es aber nicht, und die Wahrnehmung ist nicht-veridisch.

${ }^{353} \mathrm{Im}[\S 8]$ der VI. LU diskutiert Husserl kurz das etwas unthematische Identitätsbewußtsein hinsichtlich des Gegenstandes von zwei Akten (Bedeutungsintention und Bedeutungserfüllung). Dieses unthematische Identitätsbewußtsein kann das Subjekt zu dem thematischen Urteil „X ist identisch mit y“ motivieren. Ich finde, dieses Identitätsbewußtsein ist ein guter Kandidat für das noetische Pendant des bestimmbaren X, wenn wir hier auch das Prinzip des noetisch-noematischen Parallelismus verwenden wollen. Wegen des Identitätsbewußtseins hat das Subjekt also die Disposition, die Gegenstände von zwei eventuell durch Zeitspanne getrennten Akten urteilsmäßig zu identifizieren. Leider garantiert das Identitätsbewußtsein nicht, dass die Gegenstände der betreffenden Akte wirklich identisch sind, denn das Subjekt kann Gegenstände verwechseln. Beyer (2000) diskutiert diese Verwechselungsfälle und entwickelt seine eigene Interpretation des bestimmbaren X. Beyers Interpretation halte ich auch für gut motiviert. Ich vermute, dass Beyers Interpretation und meine Interpretation in eine umfassendere, einheitliche Interpretation von X vereinigt werden können. Husserls Begriff des bestimmbaren X sehe ich als eine programmatische Idee, die durch verschiedene Interpretationen in weitere Hinsichten zu differenzieren und verfeinen ist.

${ }^{354}$ Diese Kandidaten hat Husserl selber abgelehnt (in seinem Aufsatz „Intentionale Gegenstände“).

${ }^{355}$ Unsere Spezifikation des bestimmbaren X ist externalistisch und etwas disjunktivisch. Zu Argumenten dafür, dass Husserls Lehre des intentionalen Inhalts eine externalistische und disjunktivische Interpretation annimmt oder mindestens nicht ausschließt, vgl. A. David Smith, „Husserl and Externalism“, in: Synthese, Vol. 160 (2008), Springer, S.313ff. 
und Gegenstand, eine existenzneutrale, aber (auf beide Möglichkeiten) allgemein anwendbare Spezifikation des bestimmbaren X der Wahrnehmung. Auf änhliche Weise möchte ich im folgenden Paragraphen eine Spezifikation des bestimmbaren X von Urteilsakten herausarbeiten.

\section{§43. Urteilsnoema, Urteilsbedeutung, und das X in Urteilsakten}

\section{a) Das bestimmbare $X$ in Urteilsakten}

Die dynamischen Konstitutionsanalysen des Wahrnehmungsprozesses haben gezeigt, dass sich ein bestimmbares $\mathrm{X}$ in diesem Prozess konstituiert. Die jeweiligen Erscheinungen fungieren dabei als ,,perspektivische Abschattungen“356, durch die das $\mathrm{X}$ in verschiedenen Perspektiven (originär) gegeben wird. Hier konstatiert man eine dynamische Struktur, in der ein Identisches (das bestimmbare X) über eine Reihe von kognitiven Perspektivenwechseln (Erscheinungen) hinweg konstituiert wird.

Diese Struktur von Identität-in-Mannigfaltigkeit findet sich auch in Urteilsakten. Mit der Erkenntnis, dass der Sieger von Jena der Besiegte von Waterloo ist, und dass Nordamerika der drittgrößte Kontinent der Erde und der zweitgrößte Kontinent der Nordhalbkugel ist, kann ich mich auf denselben ,,Gegenstand“(,,Sachverhalt") $=\mathrm{X}$ in verschiedenen Urteilsakten beziehen: (1) „Der Sieger von Jena entdeckt den drittgrößten Kontinent der Erde“, (2) „Der Besiegte von Waterloo entdeckt den drittgrößten Kontinent der Erde“, (3) „Der Sieger von Jena entdeckt den zweitgrößten Kontinent der Nordhalbkugel“, (4) „Der Besiegte von Waterloo entdeckt den zweitgrößten Kontinent der Nordhalbkugel“. Angenommen, dass ich diese Urteilsakte nacheinander vollziehe, dann bilden diese Akte noetisch einen einheitlichen Urteilskomplex, dem noematisch derselbe „Sachverhalt“ als das Identische in verschiedenen Urteilsbedeutungen entspricht. ${ }^{357}$ Das ist auch eine Struktur von Identität $(=\mathrm{X})$ in Mannigfaltigkeit von kognitiven Perspektiven, die wir im Fall der

\footnotetext{
${ }^{356}$ Hua XI: Analysen zur passiven Synthesis, S.3.

${ }^{357}$ Hua XXVI, S.29: „In einem kategorialen Satz etwa, sagen wir „S ist p“, haben wir nicht bloß auf Seite der Subjektbedeutung zwischen dem Subjektgegenstand und der Subjektbedeutung selbst zu unterscheiden ... sondern nicht minder auch hinsichtlich des ganzen Satzes. Er sagt doch aus, daß der Subjektgegenstand die und die Beschaffenheit hat. Dies ist eine Gegenständlichkeit, eben dieser Sachverhalt, jener Gegenstand habe die und die Beschaffenheit. Dieser Sachverhalt ist solange derselbe Sachverhalt, solange eben der Gegenstand derselbe ist und die Beschaffenheit dieselbe ist und solange es dabei bleibt, daß er sie hat. Das ist aber ein gegenständlich gemeinsames in bedeutungsverschiedenen Sätzen. Derselbe Subjektgegenstand kann in bedeutungsverschiedener Weise vorgestellt sein und ebenso dieselbe Beschaffenheit. Demnach haben wir den ausgesagten Sachverhalt zu unterscheiden von der jeweiligen Weise, wie er zur Aussage kommt, nämlich in Form der verschiedenen Bedeutungen der Aussagen, die, verschiedenes besagend, doch vom selben Subjekt dieselbe Beschaffenheit aussagen."
} 
Wahrnehmung schon bemerkt haben. Genauer gesagt wird in unserem Beispiel ein identischer „Sachverhalt“ (=X oder genauer, X-Sachverhalt) über eine Reihe von mit den Bedeutungen variierenden kognitiven Perspektiven hinweg konstituiert. ${ }^{358}$

Ähnlich wie im Fall der Wahrnehmung ist die X-Konstitution von zusammenhängenden Urteilsakten keine Garantie dafür, dass den Urteilsakten ein wirklicher Sachverhalt entspricht. Im obigen Beispiel wird ein X-Sachverhalt, aber kein wirklicher Sachverhalt konstituiert, denn es ist nicht der Fall, dass Napoleon Nordamerika entdeckt hat. Doch es kann auch sein, das der Urteilsprozess ein X konstituiert, das mit dem wirklichen Sachverhalt ${ }^{359}$ zusammenfällt. Ein Historiker, der eine Biographie von Napoleon schreibt, kann die beiden Sätze „,Der Sieger von Jena wurde auf die Insel St. Helena verbannt“ und „Der Besiegte von Waterloo wurde auf die Insel St.Helena verbannt" in demselben Paragraphen schreiben. Dabei wird ein XSachverhalt konstituiert, das auch ein wirklicher Sachverhalt ist. In Analogie zu (Spezifikation des bestimmbaren X der Wahrnehmung) können wir das bestimmbare X von Urteilsakten, besonders angesichts des Verhältnisses von X und Gegenstand, wie folgt spezifizieren:

(Spezifikation des bestimmbaren X des Urteilsaktes)

In jedem Urteilsakt ${ }^{360}$ wird ein $\mathrm{X}$ konstituiert, das entweder

(i) mit dem wirklichen Gegenstand (z.B. einem Sachverhalt), der so ist, wie er im Urteilsakt intendiert ist, zusammenfällt, oder

(ii) es verhielte sich so, wie in (i) beschrieben, wenn der Urteilsakt veridisch wäre.

In unserem Beispiel variieren die kognitiven Perspektiven der Urteilsakte mit den

\footnotetext{
${ }^{358}$ Wenn man den Sachverhalt als ein bestimmbares X betrachten will, dann muss man damit rechnen, dass der Sachverhaltsbegriff vag ist. Beyer hat zwei „Stufen“ von Sachverhalt, der einer singulären Aussage entspricht, unterschieden: den W-Sachverhalt und den S-Sachverhalt. Es scheint mir, dass sowohl der W-Sachverhalt als auch der S-Sachverhalt einen X-Sachverhalt ausmachen kann. Wenn ich z.B. die beiden Aussagen (1) „,Der Kaiser von Deutschland (in referentieller Verwendung) ist in Berlin geboren“ und (2) „Der Enkel von Viktoria (in referentieller Verwendung) ist in Berlin geboren" behaupte, und zwar mit der Erkenntnis, dass der Kaiser von Deutschland und der Enkel von Viktoria eben dieselbe Person ist, die ich jetzt im Fernsehen sehe, dann fällt der X-Sachverhalt mit dem S-Sachverhalt, der bei beiden Aussagen derselbe ist, zusammen, denn in diesem Fall bin ich disponiert, zu sagen, dass ich in beiden Aussagen dasselbe behaupte. Wenn ich aber die Aussagen (1) und (2) in direkt-attributiver Verwendung der definiten Kennzeichnungen (in Beyers Terminologie) behaupte, und zwar ohne die Erkenntnis, dass beide dieselbe Person betreffen, dann scheinen die W-Sachverhalte stärkere Kandidaten für X-Sachverhalt zu sein und daher habe ich hier zwei X-Sachverhalte. Darüber vgl. Christian Beyer, Intentionalität und Referenz, §§1-2. Darüber hinaus möchte ich nicht ausschließen, dass der X-Sachverhalt mit einer Sachlage in Husserls Sinne zusammenfallen kann (Zum Begriff der Sachlage vgl. unten [§66] der vorliegenden Arbeit). Für die Frage, welche „Sachverhaltsstufe“ das bestimmbare X ausmacht, scheint es keine allgemeine, endgültige Antwort zu geben. ${ }^{359} \mathrm{Im}$ alltäglichen Sprachgebrauch spricht man vom Wirklichsein bzw. Bestehen von Sachverhalten. Husserl selber folgt auch dem Sprachgebrauch (Hua XIX/2, S.655; Hua XXVI, S.146, 148, 153) und betrachtet wirkliche Sachverhalte als ,,zum ontologischen Bestand der Welt gehörig(e)“(Süßbauer 1995, S.190, 295). Was damit genau gemeint werden kann, diskutiert Süßbauer 1995, S.190, 261, 289ff.

360 Oder in jedem Komplex von Urteilsakten, die denselben Sachverhalt intendieren oder mindestens so prätendieren.
} 
jeweiligen Urteilsbedeutungen. Im Rahmen der noetisch-noematischen Analysen identifiziert Husserl, wie oben schon erwähnt (im [§38]), den noematischen Satz des Urteilsaktes mit der Bedeutung, die der Urteilsakt einem Ausdruck verleiht, wenn er dabei als Bedeutungsintention fungiert. Nun ist es an der Zeit, auf diesen noematischen Bedeutungsbegriff einzugehen.

\section{b) Ein neuer Bedeutungsbegriff}

Nach Husserl sind Bedeutungen ideale Entitäten, und er hatte in den Logischen Untersuchungen nur eine Art idealer Entität im Auge, nämlich Spezies. Daher lag es für Husserl nahe, wie im I. Abschnitt (im [§5]) erläutert, die Bedeutungen als Spezies, die durch Bedeutungsintentionen (z.B. Urteilsakte), oder genauer, durch ihre reelle Einheit von [Materie + Qualität] instanziiert werden, zu betrachten. Das wiederholt Husserl im [§8a] der Vorlesungen über Bedeutungslehre 1908 und er bezeichnet einen solchen Bedeutungsbegriff als phänologischen oder phansischen Bedeutungsbegriff. Allerdings hat Husserl 1908 eine neue Denkweise entwickelt, um die Bedeutungen als ideale Entitäten zu konzipieren.

In den Logischen Untersuchungen macht Husserl bereits die Unterscheidung zwischen dem Gegenstand, so wie er intendiert ist, und dem Gegenstand schlechthin, welcher intendiert ist. ${ }^{361}$ Beispielsweise beziehen sich die Vorstellungen (V) „Deutschlands Kaiser“ und (V’) „,der Enkel der Königin Viktoria“ auf denselben Gegenstand schlechthin, welcher intendiert ist, jedoch der Gegenstand, so wie er intendiert ist, ist bei ihnen verschieden. Dasselbe gilt auch für Urteilsakte wie „Deutschlands Kaiser ist in Göttingen“ und „Der Enkel der Königin Viktoria ist in Göttingen“. In den Logischen Untersuchungen erklärt Husserl die Verschiedenheit von Gegenstand, so wie er intendiert ist, durch die Verschiedenheit von Bedeutungen der Bedeutungsintentionen; unter dem phänologischen Bedeutungsbegriff geht die letztere Verschiedenheit wiederum auf die der reellen Momente, oder in der Sprache von Ideen I, der noetischen Momente von Bedeutungsintentionen zurück: denn verschiedene reelle intentionale Wesen [Materie + Qualität] (oder verschiedene noetische Einheiten von [noetische Sinngebung + noetischer Setzungscharakter]) instanziieren verschiedene Bedeutungen. In dieser Weise erklärt Husserl die Verschiedenheit des Gegenstandes, so wie er intendiert ist, durch die Verschiedenheit des reellen oder

\footnotetext{
${ }^{361}$ Hua XIX/1: Logische Untersuchungen, S.414.
} 
noetischen Inhalts von Bedeutungsintentionen - die erstere verhält sich zu der letzteren wie ein Explicandum zum Explicans. ${ }^{362}$

Im Vergleich dazu versucht Husserl, im [§8b] der Vorlesungen über Bedeutungslehre 1908, die Bedeutung von Bedeutungsintentionen direkt als den Gegenstand, so wie er intendiert ist (oder den intentionalen Gegenstand als solchen, den bedeuteten Gegenstand als solchen, wie er ihn auch nennt ${ }^{363}$ ) zu konzipieren, ohne noch ein Stück weiter auf die reellen Momente der Akte zurückzugreifen:

Das Wort Bedeutung kann, scheint es, auch einen Sinn haben, der nicht nur keinen Akt, sondern auch kein Spezifisches aus dem Akt trifft, vielmehr etwas korrelativ dem Gegenüberstehendes auf gegenständlicher Seite ... Wir selbst sprechen ja im Unterschied zum Gegenstand schlechthin vom Gegenstand, so wie er bedeutet ist. Dieses Wie weist ja sicherlich hinsichtlich der Akterlebnisse auf gewisse Modi derselben $<$ hin $>$, die sich spezifisch fassen lassen; aber korrelativ entspricht dem doch auch etwas auf gegenständlicher Seite. $^{364}$

Sprechen wir gegenüber dem Gegenstand schlechthin von dem Gegenstand, genommen in der Weise, wie er bedeutet oder gedacht ist, so macht nicht nur die Weise des Denkens den Unterschied, sondern in der verschiedenen Weise des Denkens „erschauen“ wir ... eine verschiedene Weise des Gedachten als solchen. Und eben damit erwächst also in der Tat ein neuer Begriff von Bedeutung. ${ }^{365}$

Husserl will die Verschiedenheit in Bezug auf den Gegenstand, so wie er intendiert ist, noch auf die Verschiedenheit angesichts der Bedeutung zurückführen; aber nach dem Zitat versucht er die letztere Verschiedenheit nicht mehr noch ein Stück weiter durch die Verschiedenheit von Spezies der reellen Momente im Akte, sondern direkt durch ,etwas auf gegenständlicher Seite“ zu erklären. Diesen neuen Bedeutungsbegriff nennt Husserl phänomenologischen oder ontischen Bedeutungsbegriff. Dabei stellt sich die Frage, was ist dieses ,etwas auf gegenständlicher Seite“?

Für diese Frage haben Husserls Reden von ,der Gegenstand, so wie er

\footnotetext{
${ }^{362}$ Hua XXVI, S.35: ,Je nach der spezifischen Artung des Bedeutungsbewußtseins sind wir in verschiedener Weise auf Gegenständlichkeiten, und eventuell dieselben Gegenständlichkeiten, bezogen, und daran liegt es, daß wir zwischen < dem> Gegenstand schlechthin, der da bedeutet ist, und dem Gegenstand, wie er bedeutet ist, unterscheiden müssen ...“

${ }^{363}$ Ebd., S.37.

${ }^{364}$ Ebd., S.35-36.

${ }^{365}$ Ebd., S.38.
} 
intendiert/bedeutet ist“, „der intentionale/bedeutete Gegenstand als solcher" einige Spuren hinterlassen: Das sind eben die Redeweisen, mit denen Husserl in Ideen I vom Noema des Aktes spricht. Die Vermutung liegt nahe, dass dieses ,etwas auf gegenständlicher Seite" eben das Noema des Aktes ist.

Diese Vermutung bestätigt sich in Husserls Vorlesungen 1917/18 mit dem Titel „Logik und allgemeine Wissenschaftstheorie“.

\section{c) Der noematische Bedeutungsbegriff}

In Vorlesungen 1917/1918 stellt Husserl die Frage: „Wenn wir beispielsweise aussagen: „Die Winkelsumme in einem Dreieck ist gleich zwei rechten $<$ Winkeln>“ und dann fortfahren: „Dieser Satz ist ein Fundament der Euklidischen Geometrie“, worauf sind wir da mit dem Wort „Satz“ gerichtet? “ ${ }^{366}$ Mit „Satz“ meint Husserl hier, wie es aus dem Kontext hervorgeht, die Aussagebedeutung (Proposition). ${ }^{367}$

Husserl sagt explizit, er meine hier nicht die Spezies des Urteilsaktes („,Idee des Urteilens“" $)^{368}$, daher handelt es sich bei dem hier einschlägigen Begriff von Satz nicht um den phänologischen (phansischen) Bedeutungsbegriff. Im Rahmen von den zwei Bedeutungsbegriffen muss Husserl hier mit „Satz“ die Bedeutung im Sinne des phänomenologischen (ontischen) Bedeutungsbegriffs meinen.

Tatsächlich nennt Husserl den Satz abwechselnd auch das (urteilsmäßig) Vermeinte als solches, das Gesetzte als solches oder einfach Urteilsnoema (Hua XXX, 46, 52). Er glaubt, die Erforschung der Aussagebedeutung sei eben die Erforschung des Noema von dem dabei als Bedeutungsintention fungierenden Urteilsakt: „Eine rein ideale Forschung kann sich den Sätzen zuwenden, den noematischen Korrelaten der Urteile im Sinn von Noesen. “369 Darüber hinaus hat Husserl, wie schon im I. Abschnitt (Fußnote im [§22b]) erwähnt, in einer Fußnote im [§45] der Formalen und transzendentalen Logik die Urteilsbedeutung (Apophansis) mit dem Noema des Urteilsaktes identifiziert. Dort verweist er sogar den Leser auf [\$94] der Ideen I mit dem Titel „Noesis und Noema im Urteilsgebiete“. Daher stellt es sich heraus, dass die

\footnotetext{
${ }^{366}$ Hua XXX: Logik und allgemeine Wissenschaftstheorie.Vorlesungen 1917/18 mit ergänzenden Texten aus der ersten Fassung 1910/11, hrsg. von Ursula Panzer, Kluwer Academic Publishers, Dordrecht/Boston/London 1996, S.42.

367 Ebd., S.51-53.

${ }^{368}$ Ebd., S.42.

${ }^{369}$ Ebd., S.51.
} 
Bedeutung, verstanden unter dem phänomenologischen Bedeutungsbegriff, für Husserl eben das Noema der Bedeutungsintention ist. ${ }^{370}$

Deshalb bezeichnet Ursula Panzer den phänomenologischen Bedeutungsbegriff auch als noematischen Bedeutungsbegriff ${ }^{371}$; damit übereinstimmend bezeichnet Robin D. Rollinger die Bedeutung im Sinne des phänomenologischen Bedeutungsbegriffs als noematische Bedeutung ${ }^{372}$.

Urteilsbedeutung, wie schon erläutert ${ }^{373}$, gehört zum Forschungsgebiet der formalen Logik. Da sie eben Urteilsnoema ist, können wir sagen, Urteilsnoema ist eben das, was der Logiker untersucht. Doch der Logiker interessiert sich nicht für das Urteilsnoema in seinem vollen Bestand: er kümmert sich beispielsweise nicht darum, mit welchem Auffälligkeitscharakter das Urteilsnoema verbunden ist, in welchem Anschauungscharakter (evident oder nicht) es sich konstituiert usw. Der Logiker interessiert sich normalerweise für den oben im [§38] definierten noematischen Satz, nämlich die Einheit [noematischer Sinn + noematisch-thetischer Charakter der Urteilsgewißheit], die nur eine Komponente des vollen Urteilsnoema ausmacht. Manchmal ist sogar der noematisch-thetische Charakter für den Logiker irrelevant und er berücksichtigt nur den noematischen Sinn des vollen Urteilsnoema - wenn man z.B. von dem gemeinsamen Inhalt eines Urteilsaktes und eines bloßen Denkens ohne Überzeugung sprechen will. ${ }^{374}$

Zusammenfassend kann man sagen: Bedeutungen sind Noemata von Bedeutungsintentionen hinsichtlich ihrer noematischen Sätze/Sinne. Speziell sind Urteilsbedeutungen Noemata von Urteilsakten hinsichtlich ihrer noematischen Sätze/Sinne.

Was den Zusammenhang zwischen der Urteilsbedeutung, dem bestimmbaren X und evtl. dem wirklichen Gegenstand anbelangt, können wir wie folgt zusammenfassen: Der noematische Satz/Sinn als Urteilsbedeutung bestimmt die kognitive Perspektive, in der das bestimmbare $\mathrm{X}$ konstituiert wird, wobei das $\mathrm{X}$, angesichts seines Verhältnisses zum wirklichen Gegenstand (Sachverhalt), die (Spezifikation des bestimmbaren $X$ des Urteilsaktes) im [§43a] erfüllt.

\footnotetext{
${ }^{370}$ Ich nehme an, das gilt nicht nur für Urteilsnoema, sondern allgemein für Noema von Bedeutungsintentionen.

${ }^{371}$ Hua XXX: Logik und allgemeine Wissenschaftstheorie, S.XXXIV.

${ }^{372}$ Hua XL: Untersuchungen zur Urteilstheorie. Texte aus dem Nachlass (1893-1918), hrsg. von Robin D. Rollinger, Springer 2009, S.XXXIV.

${ }^{373}$ Vgl. Kapitel III im I. Abschnitt der vorliegenden Arbeit.

${ }^{374}$ Hua III/1: Ideen I, S.219.
} 


\section{§44. Debatte über Interpretation des Noema}

\section{a) West-Coast-Interpretation}

In Bezug auf das Verhältnis zwischen Noema und Gegenstand schlechthin (= wirklichem Gegenstand) kommt es zu einer berühmten Debatte zwischen der sogenannten West-Coast- und East-Coast-Interpretation. Hier kommt es uns nicht darauf an, alle Details der Debatte zu präsentieren. Diese Debatte interessiert mich insoweit, als sie zu einer vernünftig-rekonstruktiven Interpretation von Husserls Begriff des Noema führt. Um die Debatte zu skizzieren, ist es dienlich, mit der terminologischen Frage anzufangen: Wie soll man Husserls Rede von ,als solches“ in „,der (intentionale) Gegenstand als solcher“, „das Wahrgenommene als solches“" usw., mit der Husserl das Noema bezeichnet, interpretieren? Um diese Frage zu beantworten, rekurriert die West-Coast-Interpretation (Smith und McIntyre) auf Twardowski, der die Rede von ,als solches“ im Zusammenhang mit der Unterscheidung zwischen Inhalt und Gegenstand der Vorstellungen analysiert.

Twardowski wendet die Unterscheidung zwischen determinierender und modifizierender Lesart von Beiwörtern auf den Term ,,der vorgestellte Gegenstand“ an. Der Term „,die gemalte Landschaft“, wenn das Beiwort ,gemalt“ determinierend verstanden wird, bezieht sich auf eine echte Landschaft, die in einer gewissen Relation zu einem Gemälde steht; aber unter der modifizierenden Lesart bezieht er sich eben auf das Gemälde. Ähnliches gilt auch für den Term ,der vorgestellte Gegenstand“: unter einer determinierenden Lesart bezieht er sich auf den Gegenstand des Vorstellungsaktes, unter einer modifizierenden Lesart auf den (psychologischen) Inhalt des Aktes. ${ }^{375}$ Das Verhältnis zwischen dem Inhalt und dem Gegenstand beschreibt Twardowski wie folgt: „,Der Inhalt sei gleichsam das Mittel, durch welches der Gegenstand vorgestellt werde. “376

Twardowski bemerkt, dass manche Philosophen (z.B. Sigwart, Drobisch) diese Ambiguität von zwei Lesarten nicht beachten, während andere (Bolzano, Zimmermann, Kerry) sie bewusst berücksichtigen. Z.B. versucht Kerry die Ambiguität durch die Unterscheidung zwischen dem ,,Vorgestellten als solchen“ und dem ,,Vorgestellten schlechtweg“" $\mathrm{zu}$ beseitigen. ${ }^{377}$

\footnotetext{
${ }^{375}$ Kasimir Twardowski, Zur Lehre vom Inhalt und Gegenstand der Vorstellungen. Eine psychologische Untersuchung, Philosophia Verlag, München/Wien 1982, S.12-13.

${ }^{376}$ Ebd., S.18.

${ }^{377}$ Ebd., S16-19.
} 
Die West-Coast-Interpretation (im Folgenden kurz: W-Interpretation) betrachtet Husserls Rede von „das Vermeinte als solches“ oder „das vermeinte Gegenständliche, so wie es vermeint ist" vor diesem historischen Hintergrund. Denn Twardowski war Husserls Zeitgenosse und auch ein Schüler von Brentano; Husserl ist vertraut mit Twardowskis Zur Lehre vom Inhalt und Gegenstand der Vorstellungen und hat darüber eine Rezension 378 geschrieben; in Ideen $I$ ([§129] mit dem Titel: „Inhalt“ und „Gegenstand“; der Inhalt als „Sinn“) hat Husserl Twardowkis „schöne Abhandlung“ und „Verdienst“ 379 gewürdigt; außerdem hat Husserl in seinem Aufsatz „Intentionale Gegenstände“ die Unterscheidung zwischen determinierender und modifizierender Attribution explizit erwähnt und sie auf Twardowski zurückgeführt. ${ }^{380}$ Demzufolge vertreten Smith und McIntyre die Ansicht, dass Husserl Twardowskis Schema von Akt-Inhalt-Gegenstand akzeptiert und dass Husserls Rede von „der (intentionale) Gegenstand als solcher“, „das Wahrgenommene als solches" usw. in modifizierender Lesart zu verstehen ist. ${ }^{381}$ Das bedeutet, das Noema ist nicht der intendierte Gegenstand, sondern der Inhalt, durch den der Akt seinen Gegenstand intendiert.

Der Unterschied zwischen Husserls und Twardowskis Akt-Inhalt-GegenstandSchema besteht darin, so die W-Interpreten, dass Husserls Inhalt ideale Entität ist, während Twardowskis Inhalt wohl als psychologisches Datum verstanden werden könnte. ${ }^{382}$ Daher vertritt Husserl ein Akt—idealer-Inhalt—Gegenstand-Schema von Intentionalität. Das Noema, als idealer Inhalt, hat keine raumzeitlichen, physischen Eigenschaften. Die W-Interpretation vergleicht Husserls Begriff des Noema mit Freges Begriff des Sinnes: Beide sind ideale Entitäten, durch die der Akt (oder bei Frege, der Ausdruck) sich auf den Gegenstand bezieht. Nach der W-Interpretation versteht Husserl seit Ideen I die Idealität des intentionalen Inhalts nicht mehr als Spezies ${ }^{383}$, daher nennen Smith und McIntyre das Noema manchmal auch abstrakte Einzelheit (abstract particular).

Für die W-Interpretation ist der Unterschied zwischen Noema und Gegenstand im

\footnotetext{
${ }^{378}$ Hua XXII: Aufsätze und Rezensionen, S.349-356.

${ }^{379}$ Hua III/1: Ideen I, S.298.

${ }^{380}$ Hua XXII: Aufsätze und Rezensionen, S.309.

${ }^{381}$ David Woodruff Smith and Ronald McIntyre, Husserl and Intentionality, S.109-112, 162-165.

${ }^{382}$ Ebd., S.112-119.

${ }^{383}$ Smith und McIntyre zufolge hat Husserls Konzeption der Idealität von intionalem Inhalt sich verändert: In den Logischen Untersuchungen ist der intentionale Inhalt als Spezies angesehen, aber ab Ideen I versteht Husserl ihn als ideale Entität von anderer Art, die keine Spezies ist. Vgl. Smith, S.124-125.
} 
Fall der Wahrnehmung besonders klar: Das Wahrnehmungsnoema ist etwas Ideales, es hat keine raumzeitlichen und physischen Eigenschaften; der intendierte Wahrnehmungsgegenstand, als physisches Ding, befindet sich in Raum und Zeit und hat mannigfaltige physische Eigenschaften; der Unterschied zwischen den beiden ist ein ontologischer Unterschied. Mit dieser ontologischen Unterscheidung fällt es der WInterpretation leicht, das berühmte Zitat aus Ideen I zu interpretieren:

Der Baum schlechthin, das Ding in der Natur, ist nichts weniger als dieses Baumwahrgenommene als solches, das als Wahrnehmungssinn zur Wahrnehmung und unabtrennbar gehört. Der Baum schlechthin kann abbrennen, sich in seine chemischen Elemente auflösen usw. Der Sinn aber Sinn dieser Wahrnehmung, ein notwendig zu ihrem Wesen Gehöriges - kann nicht abbrennen, er hat keine chemischen Elemente, keine Kräfte, keine realen Eigenschaften. ${ }^{384}$

Da die beiden ontologisch verschiedene Entitäten sind, wundert es nicht, dass der Baum schlechthin nichts weniger ist als das Baumwahrgenommene als solches.

Eine ausführliche Darstellung der W-Interpretation würde die Grenzen der vorliegenden Arbeit überschreiten, hier genügt es, die Stichpunkte der W-Interpretaion hervorzuheben:

(1) Das Noema ist eine ideale oder abstrakte Entität, d.h. es hat keine raumzeitlichen und physischen Eigenschaften; im Gegensatz dazu ist der Gegenstand in vielen Fällen etwas Reales.

(2) Der Akt intendiert den Gegenstand, indem er das Noema unterhält (entertain) ${ }^{385}$, denn das Noema bestimmt, auf welchen Gegenstand der Akt gerichtet wird, und zwar in welcher Weise er ihn intendiert.

(3) Um ein Missverständnis zu vermeinden, betont die W-Interpretation: Die vermittelnde Rolle des Noema zwischen Akt und Gegenstand heißt nicht, dass der Akt zunächst das Noema unmittelbar und dann den Gegenstand mittelbar intendiert, wie es im Zeichen- oder Bildbewußtsein der Fall ist. Metaphorisch gesagt ist das Noema nur Instrument, aber kein Ziel der Intentionalität. Mit einem Wort, der Akt intendiert nicht

\footnotetext{
${ }^{384}$ Hua III/1: Ideen I, S.205.

${ }^{385}$ Smith und McIntyre benutzen den Term „entertain“, um das Verhältnis zwischen Akt und Noema zu bezeichnen. Sie glauben, dass das Entertaining-Verhältnis bei Husserl ein Grundbegriff ist und sich nicht weiter analysieren lässt. Vgl. David Woodruff Smith and Ronald McIntyre, Husserl and Intentionality, S.144.
} 
das Noema. ${ }^{386}$

Die W-Interpretation ist nicht ohne Schwierigkeiten. Es ist zuzugestehen, dass Husserl in Ideen I manchmal das Noema einfach Sinn ${ }^{387}$ nennt und den Sinn als ideale Entität betrachtet sowie von realen Gegenständen unterscheidet ${ }^{388}$. Aber nach der Einführung des bestimmbaren $\mathrm{X}$ scheint ein absoluter Unterschied zwischen Noema und Gegenstand ins Wanken zu geraten. Denn einerseits scheidet sich das X, wie gesagt, als ,innerstes“ und „,zentrales noematisches“ (Hua III/1, S.299, 302) Moment aus, andererseits bezeichnet Husserl das $\mathrm{X}$ als Gegenstand-in-Anführungszeichen (oder ,,Gegenstand schlechthin “389). Die Anführungszeichen deutet darauf hin, dass die Existenz oder Nicht-Existenz des Gegenstandes hier innerhalb der phänomenologischen Reduktion ausgeklammert wird. Aber die Möglichkeit, dass das $\mathrm{X}$ mit einem wirklichen Gegenstand identisch ist, wird dabei nicht ausgeschlossen, sondern bleibt nur außer Betracht. An manchen Textstellen, wo Husserl den Wahrnehmungsprozess diskutiert, behandelt er das X sogar direkt als ein wirkliches Ding (ohne Anführungszeichen), wobei er die Voraussetzung, dass der Wahrnehmungsprozess veridisch ist, hätte explizit machen sollen. Zum Beispiel ${ }^{390}$ :

Keine Wahrnehmung des Dinges ist letztabgeschlossene ... Mit jedem Fortgange bereichert sich der Bestimmungsgehalt des Dingnoemas, das stetig zu demselben Dinge X gehört.

Ein individuelles Ding anschauend ... vollziehen wir Kontinuen des Anschauens ... der Blick ist dabei auf das Identische, auf das X des Sinnes gerichtet ... auf das eine und selbe, das sich verändert, dreht usw.

In jedem Moment ist der gegenständliche Sinn derselbe hinsichtlich des Gegenstandes schlechthin, der gemeinter ist, und ist in der kontinuierlichen Abfolge der Momentanerscheinungen in Deckung. So etwa dieser Tisch da. Aber dieses Identische ist ein beständiges $\mathrm{x}$, ist ein beständiges Substrat von wirklichen erscheinenden Tisch-Momenten, aber auch von Hinweisen auf noch nicht erscheinende.

Das alles impliziert, dass das Noema in Bezug auf sein eigenes Moment des X mit dem intendierten (evtl. realen) Gegenstand des Aktes identisch sein kann.

\footnotetext{
${ }^{386} \mathrm{Ebd}$.

${ }^{387}$ Hua III/1: Ideen I, S.203, 206, 217.

${ }^{388}$ Vgl. Dagfinn Føllesdal, „Husserl’s Notion of Noema“, in: Husserl, Intentionality and Cognitive Science, hrsg. von Hubert L. Dreyfus und Harrison Hall, The MIT Press, Cambridge Massachusetts/London 1982, S.77.

${ }^{389}$ Hua III/1: Ideen I, 303.

${ }^{390}$ Ebd., S.331, 347, 349 und Hua XI: Analysen zur passiven Synthesis, S.5.
} 
Wir können sagen, (i) dass Husserl das Noema, besonders im Hinblick auf den noematischen Satz/Sinn, für etwas Ideales hält, (ii) während er nicht ausschließt, dass das Noema angesichts des noematischen $\mathrm{X}$ mit realem Gegenstand zusammenfallen kann.

Die W-Interpretation ignoriert diese in Husserls Texten implizierte partielle Indentitätsmöglichkeit zwischen Noema und (evtl. realem) Gegenstand und versucht das noematische $\mathrm{X}$ ausschließlich als einen besonderen idealen Sinn, der eindeutig für den (evtl. realen) Gegenstand steht, zu betrachten ${ }^{391}$. Aus diesem Grund betont sie einseitig Punkt (i) und unterlässt Punkt (ii). Im Gegensatz dazu hat die East-CoastInterpretation (Drummond) Punkt (ii) betont und sogar überbetont. Dieser Interpretation wollen wir uns jetzt zuwenden.

\section{b) East-Coast-Interpretation}

Nach der East-Coast-Interpretation (im Folgenden kurz: E-Interpretation) soll die Rede „der intentionale Gegenstand als solcher“ in der normalen Lesart, d.h. der determinierenden Lesart ${ }^{392}$ verstanden werden: Der intentionale Gegenstand als solcher (das Noema) ist eben der intendierte Gegenstand. ${ }^{393}$ Dieses Identitätsverhaltnis zwischen Noema und Gegenstand bildet die Grundannahme der E-Interpretation.

Der Beisatz „als solches“ in Verbindungen wie ,,der intentionale Gegenstand als solcher“, „das Wahrgenommene als solches“ usw. dient für Drummond nur dazu, auf die Verschiedenheit zwischen der phänomenologischen und natürlichen Betrachtungsweise in Bezug auf ein und denselben Gegenstand zu verweisen. In der natürlichen Betrachtungsweise interessiert man sich für die physischen Eigenschaften des Baumes und fragt z.B., ob der Baum verbrennbar ist; in der phänomenologischen Betrachtungsweise kümmert man sich nur um die kognitive Zugänglichkeit (cognitive availability $^{394}$ ) ein und desselben Gegenstandes, d.i. die Bewußtseinsweise, in der der Gegenstand als Korrelat des Aktes gegeben ist. Dabei sind Prädikate wie ,verbrennbar“ irrelevant. Die E-Interpretation glaubt, dass wir das im letzten

\footnotetext{
${ }^{391}$ David Woodruff Smith and Ronald McIntyre, Husserl and Intentionality, S.200-222.

392 „Here the expression "the perceived as such" is introduced as explanatory of the meaning of "perceptual sense", and the ordinary meaning of the expression "the perceived as such" refers clearly to the perceived object just insofar and how it is perceived ... it becomes even more clear that the "as such" formulations do, in fact, refer to the intended objectivity itself ... "John Drummond, Husserlian Intentionality and Non-Foundational Realism. Noema and Object, Kluwer Academic Publishers, Dordrecht/Boston/London 1990, S.117-118.

393 „The object, the sense, and the noema are the same differently considered.“ Vgl. Ebd., S.113-117, 122.

394 Dan Zahavi, Husserl's Legacy. Phenomenology, Metaphysics, and Transcendental Philosophy, Oxford University Press, Oxford 2017, S.91.
} 
Paragraphen angeführte Zitat (,der Baum schlechthin ... “) interpretieren können, ohne eine ontologische Unterscheidung zwischen dem Baum schlechthin und dem BaumNoema zu machen. Denn Fragen, die die realen Eigenschaften des Baumes betreffen, gehören gar nicht zur phänomenologischen Betrachtungsweise: Es macht für den Phänomenologen keinen Sinn, Fragen wie „Ist das Baum-Noema verbrennbar?“ zu beantworten, wenn er dies tut, dann verlässt er die Phänomenologie und bewegt sich eigentlich in der Physik. ${ }^{395}$

Im Vergleich zu W-Interpretation können wir zwei Punkte der E-Interpretation hervorheben: (1) Noema und Gegenstand sind identisch, man unterscheidet sie terminologisch, nur um verschiedene Betrachtungsweisen für ein und denselben Gegenstand zu kennzeichnen; (2) Wegen (1) kann es kein ontologischer Unterschied zwischen den beiden bestehen. Konsequenterweise vertritt die E-Interpretation die Ansicht, dass das Noema der (veridischen) Wahrnehmung eben der Wahrnehmungsgegenstand und somit ontologisch etwas Reales ist.

Um die Grundannahme des Identitätsverhältnisses zwischen Noema und Gegenstand $\mathrm{zu}$ begründen, rekurriert Drummond auf Husserls Begriff des bestimmbaren X. Wie oben schon erwähnt, Husserl bestimmt einerseits das bestimmbare $\mathrm{X}$ als ein Moment des Noema, andererseits identifiziert er das $\mathrm{X}$ an manchen Textstellen direkt als den intendierten (evtl. realen) Gegenstand. Das verwertet Drummond für die These, dass der intendierte Gegenstand mit dem Noema, oder genauer, mit dem innersten Moment $(=\mathrm{X})$ des Noema identisch ist. ${ }^{396}$ Wenn Husserl sagt, dass sich das Bewußtsein durch Noema auf den Gegenstand bezieht, dann meint er nicht, so Drummond, dass das Bewußtsein mittels des Noema auf einen vom Noema verschiedenen Gegenstand gerichtet wird, sondern dass das Bewußtsein innerhalb des Noema durch den mit verschiedenen Charakteren verschmolzenen noematischen Sinn bis in das innerste Moment X durchdringt. ${ }^{397}$

\footnotetext{
395 „One does not predicate of the perceived object simply as perceived, i.e. the perceptual appearance of the object upon which we reflect philosophically, what one predicates of the perceived object itself which we experience straightforwardly, because the kinds of consideration given to the two necessarily differ." John Drummond, Husserlian Intentionality and Non-Foundational Realism, S.116. Vgl. auch John Drummond, „The Doctrine of the noema and the theory of reason“, in: Commentary in Husserl's Ideas I, hrsg. von Andrea Staiti, Walter de Gruyter, Berlin/Boston 2015, S.263.

396 „Smith and McIntyre ... committed to the view that the noema is a Fregean sense, must understand ... the " $\mathrm{X}$ " along the lines of linguistic meaning ... Hence, they understand it along the lines of a demonstrative pronoun. I have suggested, however, that this view conflicts with Husserl's texts, since the texts identify " $X$ " as the intended objectivity itself:“ John Drummond, Husserlian Intentionality and Non-Foundational Realism, S.108, 135.

${ }^{397}$ Ebd., S.136: ,The language of "through", therefore, is not instrumental in the sense that the noematic Sinn is an instrumental entity distinct from the intended object and an avenue of access to that object such that we go through and beyond the Sinn to the object. Instead we go through the Sinn in the sense that we penetrate it. The intended objectivity is contained within the noema just as it is intended, and the determinable " $\mathrm{X}$ " is that object considered
} 
Die E-Interpretation ist auch nicht ohne Schwierigkeiten. Das Noema, wie wir schon wissen, enthält verschiedene Momente, wobei das X nur eines davon ausmacht. Auch wenn wir Drummonds These, dass das bestimmbare $\mathrm{X}$ der Wahrnehmung mit dem Wahrnehmungsgegenstand zusammenfällt und also etwas Reales ist, akzeptieren, ${ }^{398}$ heißt das bei weitem nicht, dass andere noematische Momente wie der noematische Sinn und sogar das volle Noema etwas Reales sind. Bei Bedeutungsintentionen ist der Kontrast besonders klar: Nach dem noematischen Bedeutungsbegriff sind noematische Sätze/Sinne von Bedeutungsintentionen eben ihre Bedeutungen; und Bedeutungen (wie ,,der Sieger von Jena“) sind für Husserl ideale Entitäten, während die Gegenstände, auf die sie sich beziehen, oft reale Entitäten (wie die Person Napoleon) sind; hier enthält das Noema von Bedeutungsintentionen sowohl ideale als auch reale Entitäten. Wie kann Drummond, so fragt man sich, von der idealen Natur des noematischen Satzes/Sinnes absehend, nur die reale Natur des $\mathrm{X}$ betonen und einfach behaupten, dass das (volle) Noema mit dem realen Gegenstand identisch und deshalb auch etwas Reales ist?

Drummonds Antwort, soviel ich weiß, lautet: In der ersten Auflage der Logischen Untersuchungen wird der Unterschied zwischen Bedeutung und (realem) Gegenstand als ontologischer Unterschied verstanden, aber seit Ideen I ist es nicht klar, ob Husserl ihn noch ontologisch versteht. ${ }^{399}$ Seiner Meinung nach heißt ,ideal“ seit Ideen I nur ,nicht-reell“ und nichts mehr. ${ }^{400}$ Drummond versucht, die Unterscheidung von Realem und Idealem zu de-ontologisieren, um das volle Noema, einschließlich des noematischen Sinnes und des bestimmbaren X, als eine ontologisch homogene Einheit betrachten zu können. Diesen Versuch kann ich nur für falsch halten ${ }^{401}$, denn selbst in seinem Spätwerk Formale und transzendentale Logik betont Husserl noch die ontologische Besonderheit des Idealen:

Sie [Urteilsbedeutungen] sind eben keine realen, keine Raumgegenstände, sondern irreale Geistesgebilde, deren eigentümliches Wesen die räumliche

\footnotetext{
formally, apart from its determinations.“

${ }^{398}$ Unter der Voraussetzung, dass die Wahrnehmung veridisch ist.

${ }^{399}$ John Drummond, Husserlian Intentionality and Non-Foundational Realism, S.124: „We have already seen that in the first edition of LU, the distinction between the sense as an ideal species which is instantiated in acts and the referent of expression is clearly ontological. But it is not clear that this view of the distinction between sense and intended objects ... endures in Ideen I and later works.“

${ }^{400}$ Ebd., S.174: „Not every ideal reality must be considered a reality ontologically distinct from the real, worldly object. Relative to an experience, the worldly object insofar as it is the correlate of that experience is ideal, in the sense of "ir-real", precisely because it is not really contained within the experience."

${ }^{401}$ Diesen Fehler von Drummond bemerkt auch Łukasz Kosowski. Sieh Łukasz Kosowski, Noema and Thinkability. An Essay on Husserl's Theory of Intentionality, Ontos Verlag, Heusenstamm 2010, S.110.
} 
Extension, ursprüngliche Örtlichkeit und Beweglichkeit, ausschließt. Wie andere Geistesgebilde lassen sie aber eine physische Verleiblichung zu, hier die durch die sinnlichen Wortzeichen, und gewinnen so ein sekundäres räumliches Dasein ... Jede Art Irrealität, von der die Idealität der Bedeutungen und die von ihr zu scheidende Idealität der allgemeinen Wesen oder Spezies besondere Fälle sind, hat Weisen möglicher Anteilhabe an der Realität. Aber das ändert nichts an der prinzipiellen Sonderung zwischen Realem und Irrealem. $^{402}$

Eine phänomenologische Spezifikation des bestimmbaren X muss, wie oben schon erläutert, in Bezug auf den intendierten Gegenstand existenzneutral sein. Aber Drummond orientiert sich bei seiner Analyse des bestimmbaren X hauptsächlich nach den veridischen Fällen. Das sind Fälle, die für seine These günstig sind, und er unterlässt die nichtveridischen Fälle, die er auch hätte berücksichtigen sollen. Z.B. bei seiner Wahrnehmungsanalyse identifiziert Drummond das X einfach mit dem realen Wahrnehmugnsgegenstand, ohne diese Identifizierung durch die dabei verborgene Voraussetzung, dass die Wahrnehmung veridisch ist, zu beschränken. Man möchte Drummond fragen, mit was kann das $\mathrm{X}$ identisch sein, wenn die Wahrnehmung eigentlich Halluzination ist? Mit einem realen, aber nicht-existenten Gegenstand? ${ }^{403}$

\section{c) Quelle der Schwierigkeiten und ihre Überwindung}

Beide Interpretationen stehen vor Schwierigkeiten: Die W-Interpretation betont den ontologischen Unterschied zwischen idealen Sätzen/Sinnen und realen Gegenständen und ignoriert die eventuelle Identität zwischen dem noematischen X und dem realen Gegenstand (wenn der Gegenstand existiert); im Gegensatz dazu hat die EInterpretation die eventuelle Identität zwischen $\mathrm{X}$ und realem Gegenstand überbetont und die Idealität der noematischen Sätze/Sinne, auf der Husserl immer besteht, unterlassen.

Meines Erachtens besteht die Quelle der Schwierigkeiten darin, dass beide Parteien die Tendenz haben, das Noema als eine ontologisch homogene Einheit zu betrachten: Die W-Interpretation geht von den idealen noematischen Sätzen/Sinnen aus und erweitert diese Idealität auf das volle Noema, während die E-Interpretation von der

\footnotetext{
${ }^{402}$ Hua XVII: Formale und transzendentale Logik, §57b.

${ }^{403}$ Für weitere Kritiken über Drummond vgl. Łukasz Kosowski, Noema and Thinkability, S.99, 103-104, 106-110.
} 
eventuellen Identität zwischen X und realem Gegenstand ausgeht und diese Identität auf das volle Noema extendieren will.

Ich sehe keinen Grund, warum man das Noema für eine ontologisch homogene Einheit halten muss. In den Logischen Untersuchungen hat Husserl das intentionale Wesen in specie, das etwas Ideales ist, und den intentionalen Gegenstand (im Sinne von intendiertem Gegenstand), der oft etwas Reales ist, unter den Titel ,intentionaler Inhalt“ zusammengebracht; ${ }^{404}$ Seit Ideen I nennt Husserl den intentionalen Inhalt Noema $;{ }^{405}$ daher schließt Husserl eigentlich nicht aus, dass der intentionale Inhalt (=das Noema in Ideen I) ontologisch verschiedene Entitäten umfassen kann. In der analytischen Philosophie gibt es ähnliche Thesen, die ontologisch verschiedene Entititäten unter den Titel „Proposition“ bringen. ${ }^{406}$ Hinzu kommt, dass ein Autor über Husserl, David Bell, die innere Heterogeneität des Noema (und der Noesis) betont:

The concept of a noesis is not a sortal concept; and in answer to the question 'What is a noesis?' we have to say: It's anything and everything that can possibly occur as a real part or moment of an intentional experience - with the exception of hyletic data. The term 'noesis', in other words, signifies merely a ragbag concept; it is not a name for some specific sort of thing or kind of activity.

But if we take seriously the claim that noemata are in every respect isomorphic with the correlative noeses, and if the concept of a noesis is merely a ragbag concept in whose extension belong all sorts of radically different kinds of things, then we should resist the temptation to think that a noema is some particular sort of object, or that the concept of a noema is a genuine sortal concept ... The concept of a noema is a ragbag concept too. ${ }^{407}$

David Bell diagnostiziert die Versuchung, das Noema als eine homogene Einheit zu interpretieren, als die Konsequenz einer sprachlichen Fehlanwendung des scheinbar singulären Terms ,Noema“:

Now Husserl ... wants to know what makes an experience significant; but unfortunately he then introduces a singular term, 'the noema', whose function

\footnotetext{
${ }^{404}$ Hua XIX/1: Logische Untersuchungen, S.413.

${ }^{405}$ Vgl. [§32] der vorliegenden Arbeit.

406 Jeffrey King hat verschiedene Bilder/Theorien von Propositionen dargestellt. Dabei erwähnt er Russells und die neo-Russellsche Metaphysik von Propositionen. In solchen metaphysischen Bildern bestehen manche Propositionen (d.i. Propositionen, die durch singuläre Aussagen wie „Sokrates ist sterblich“ ausgedrückt werden) aus konkreten Objekten und abstrakten Begriffen - ein ontologisch heterogenes Bild von Propositionen. Dazu vgl. Jeffrey C. King, „The Metaphysics of Propositions“, in: Oxford Handbooks Online (www.oxfordhandbooks.com).

${ }^{407}$ David Bell, Husserl, S.175, 179-181.
} 
is in effect to stand for what makes an experience significant. But there is no such thing: the italicized phrase is spurious singular term.

Konklusion 408 : Der Begriff des Noema ist, wie Bell bemerkt, ein Sammelsuriumsbegriff (ragbag concept), der auf Entitäten von ontologisch heterogenen Kategorien anwendbar ist.

Bernet ist der Meinung, dass Husserls Begriff des Noema vieldeutig ist: manchmal ist Noema die ideale Bedeutung, manchmal der reale Gegenstand, usw. Meiner Meinung nach ist diese „Vieldeutigkeit“ kein Mangel des Noemabegriffs, sondern sie spiegelt eben die ontologische Vielfalt der noematischen Komponenten. Etwas metaphorisch gesprochen, das Noema ist kein reines Metall, sondern eine Legierung.

Wir können das Noema als ein Feld von Gegebenheiten verstehen. In diesem Feld befinden sich ideale noematische Sätze/Sinne und das $\mathrm{X}$, das eventuell ein realer Gegenstand ist, und zwar in Verbindung mit bestimmtem Auffälligkeitscharakter ([§36]) und Anschauungscharakter ([§35]). Wir können das Noema durch das Schema I[(Sinn,

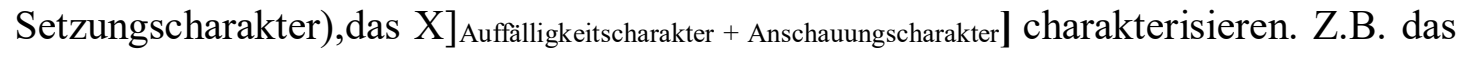
noematische Feld meiner Baum-Wahrnehmung lässt sich durch I[(,grüner Baum“,

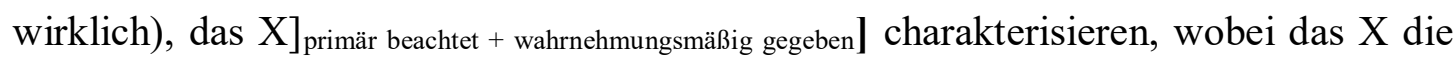
(Spezifikation des bestimmbaren X der Wahrnehmung) im [§42c] erfüllt.

In diesem Schema steht „Sinn“/,,(Sinn, Setzungscharakter)“ für noematischen

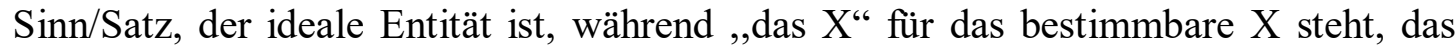
eventuell, aber nicht notwendig mit einem realen Gegenstand zusammenfällt. Hier erlauben wir die ontologische Heterogenität der Komponenten innerhalb des Noema und deshalb vermeiden die gemeinsame Tendenz von W- und E-Interpretation.

Auf ähnliche Weise können wir auch das noematische Feld des Urteilsaktes charakterisieren. Z.B. lässt sich das Noema meines Urteilsaktes „der Sieger von Jena ist Soldat" durch $[[($, ,der Sieger von Jena ist Soldat“, wirklich), das X]...] charakterisieren, wobei das bestimmbare $\mathrm{X}$ die (Spezifikation des bestimmbaren $\mathrm{X}$ des Urteilsaktes) im [§43a] erfüllt.

\section{§45. Zusammenfassung des Kapitels}

Im vorliegenden Kapitel beschäftigte uns die dynamische Konstitutionsanalyse.

\footnotetext{
${ }^{408}$ David Bells Begründung dafür ist für mich nicht zwingend, denn er scheint den Paralellimus zwischen Noesis und Noema zu übertreiben. Doch die Konklusion haben wir schon oben, unhängig von David Bell, begründet.
} 
Dabei bemerkten wir, dass Husserls Begriff des bestimmbaren X in der dynamischen Analyse eine wichtige Rolle spielt ([§40]). Wir beschrieben, wie das bestimmbare $\mathrm{X}$ in einer synthetischen Einheit von Einzelwahrnehmungen konstituiert wird ([§41]). Dann haben wir mit Rücksicht auf das Verhältnis zwischen X und Wahrnehmungsgegenstand eine existenzneutrale Spezifikation des X der Wahrnehmung entwickelt ([§42]). In analoger Weise haben wir auch die Konstitution des bestimmbaren X von Urteilsakten analysiert und eine existenzneutrale Spezifikation des X von Urteilsakten entwickelt $([\S 43])$.

Das Thema des bestimmbaren X führte uns zur Debatte zwischen der W- und EInterpretation des Noema ([§44]). Wir haben gezeigt, dass beide Interpretationen ihre Schwierigkeiten haben. Diese Schwierigkeiten stammen aus ihrer gemeinsamen Tendenz, das Noema immer als eine ontologisch homogenene Einheit zu betrachten. Deshalb schlug ich vor, zu akzeptieren, dass das Noema ein ontologisch-heterogene Komponenten umfassendes Feld sein kann.

Mithilfe des von uns rekonstruierten Noemabegriffs haben wir nun das Instrumentarium, die Grammatikalitätsthesen, die wir im I. Abschnitt der vorliegenden Arbeit entwickelt haben, in die transzendentale Phänomenologie zu transplantieren. Das ist die Aufgabe des folgenden Kapitels. 


\section{KAPITEL}

\section{REINTERPRETATION DER GRAMMATIKALITÄTSTHESEN IM}

\section{RAHMEN DER TRANSZENDENTALEN PHÄNOMENOLOGIE}

\section{§46. Formenlehre der noematischen Sätze/Sinne}

In Ideen I gibt es nicht viele Textstellen, an denen Husserl von der Formenlehre der Bedeutungen spricht. ${ }^{409}$ Zwei wichtige Paragraphen davon sind [§133] und [§134]. In den beiden Paragraphen greift Husserl auf seinen noematischen Bedeutungsbegriff zurück, um das Forschungsgebiet der Formenlehre der Bedeutungen in den noematischen Inhalt des Aktes einzubetten.

Im [§134] spricht Husserl in einem allgemeinen Sinne von Formenlehre der Sätze/Sinne. Mit „Sinn“ ist hier der noematische Sinn und mit „Satz“ der oben im [§38] definierte noematische Satz gemeint. Der Begriff noematischer Satz/Sinn im allgemeinsten Sinne findet seine Anwendung, wie im [§38] schon erwähnt, nicht nur bei objektivierenden Akten, die die bedeutunggebende oder bedeutungerfüllende Funktion übernehmen können, sondern auch bei nicht-objektivierenden Akten - alle Akte haben noematischen Satz/Sinn. In der vorliegenden Arbeit interessieren wir uns hauptsächlich für objektivierende Akte. Innerhalb der Gattung von objektivierenden Akten bilden Ausdrucksbedeutungen nur eine Sonderklasse der noematischen Sätze/Sinne, denn nach Husserls noematischem Bedeutungsbegriff $([\S 43 \mathrm{c}])$ sind sie nur noematische Sätze/Sinne von bedeutunggebenden Akten (Bedeutungsintentionen).

Die Formenlehre der Bedeutungen, mit der wir uns im I. Abschnitt ausführlich beschäftigt haben, ist nach Husserls noematischem Bedeutungsbegriff eine Formenlehre in Bezug auf noematische Sätze/Sinne von Bedeutungsintentionen. Wenn Husserl in Ideen I in einem allgemeinen Sinn von Formenlehre der (noematischen) Sätze/Sinne spricht, hat er tatsächlich die Idee der Formenlehre erweitert. Denn dabei meint er eine Formenlehre, die sowohl die noematischen Sätze/Sinne von Bedeutungsintentionen als auch die von bedeutungerfüllenden Akten angesichts der grammatischen Formen untersucht. (Wir sehen hier vorläufig von nichtobjektivierenden Akten ab.) Aber diese Erweiterung bedarf einer Rechtfertigung.

\footnotetext{
${ }^{409}$ Hua III/1: Ideen I, §§11, 121, 133-134, 147.
} 
In $I V . L U$ zeigt Husserl nur, dass jede Bedeutung einer Bedeutungskategorie untersteht und gewissen durch die Bedeutungskategorie bestimmten grammatischen Gesetzen unterworfen ist. Die Bedeutungskategorien und die durch sie bestimmten grammatischen Gesetze untersucht die Formenlehre der Bedeutungen. Streng genommen ist also die Formenlehre, die in $I V . L U$ und Formale und transzendentale Logik konzipiert wurde, nur eine Formenlehre der noematischen Sätze/Sinne von Bedeutungsintentionen. Um die Anwendung der Formenlehre auf bedeutungerfüllende Akte gerechtfertigt zu erweitern, muss man zeigen, dass auch die noematischen Sätze/Sinne von bedeutungerfüllenden Akten gewissen formalen Kategorien unterstehen und den durch diese Kategorien bestimmten Gesetzen unterworfen sind. Darüber hat Husserl leider in Ideen I in großem Maße geschwiegen.

Jedoch hat er einen kleinen Hinweis hinterlassen. An vereinzelten Stellen ${ }^{410}$ der Ideen I spricht Husserl von den in einem bedeutungerfüllenden Akt (wie Wahrnehmung) implizierten syntaktischen Operationen, die in höheren Stufen des bedeutungerfüllenden Aktes (wie Wahrnehmung) stattfinden können. Durch solche Operationen erwachsen syntaktisch geformte noematische Sätze/Sinne. Genauer gesprochen unterstehen die dabei erwachsenen noematischen Sätze/Sinne formalen Kategorien, die den Bedeutungskategorien der noematischen Sätze/Sinne von Bedeutungsintentionen parallel entsprechen. Deshalb können wir sagen, dass auch die noematischen Sätze/Sinne von bedeutungerfüllenden Akten gewissen formalen Kategorien unterstehen und den durch diese Kategorien bestimmten Gesetzen unterworfen sind. Dann ist die fragliche Erweiterung gerechtfertigt. Die genetischphänomenologischen Analysen in Erfahrung und Urteil beschreiben, wie diese Operationen stattfinden und somit die noematischen Sätze/Sinne gemäß den zu Bedeutungskategorien parallelen Formen strukturieren. Darüber werden wir im III. Abschnitt der vorliegenden Arbeit ausführlich diskutieren. Provisorisch nehmen wir an, dass wir die Rede von einer allgemeinen Formlehre von noematischen Sätzen/Sinnen schon gerechtfertigt haben.

\footnotetext{
${ }^{410}$ Hua III/1: Ideen I, $\S \S 124,134,153$.
} 


\section{§47. Reinterpretation der Grammatikalitätsthese der Bedeutungen im Rahmen der noetisch-noematischen Analysen}

Sehen wir provisorisch von bedeutungerfüllenden Akten ab und konzentrieren uns zunächst auf Bedeutungsintentionen. Im I. Abschnitt haben wir die Grammatikalitätsthese der Bedeutungen aufgestellt und die Rede des Grammatikalitätsphänomens der Bedeutungen eingeführt. Zur Erinnerung:

(Die Grammatikalitätsthese der Bedeutungen)

Ausdrucksbedeutungen sind gewissen durch Bedeutungskategorien

bestimmten grammatischen Gesetzen unterworfen. Das bezeichnen wir als das

Grammatikalitätsphänomen (bezüglich) der Bedeutungen.

Nach Husserls noematischem Bedeutungsbegriff sind Ausdrucksbedeutungen, wie schon erläutert, eben die noematischen Sätze/Sinne von Bedeutungsintentionen. Daher ist das Grammatikalitätsphänomen bezüglich der Bedeutungen eigentlich das Grammatikalitätsphänomen bezüglich der noematischen Sätze/Sinne von Bedeutungsintentionen:

(1) Einerseits sind Bedeutungskategorien eigentlich Formen der noematischen Sätze/Sinne (von Bedeutungsintentionen). Konsequenterweise ist die Unterscheidung von selbständiger/unselbständiger Bedeutung eigentlich eine Unterscheidung zwischen selbständigem und unselbständigem noematischen Satz/Sinn. Selbständige noematische Sätze/Sinne sind substantivische und propositionale Sätze/Sinne, unselbständige noematische Sätze/Sinne sind z.B. adjektivisch, konjunktivisch usw. Daher können wir die Bedeutungskategorien auch als grammatisch-noematische Kategorien (der noematischen Sätze/Sinne von Bedeutungsintentionen) bezeichnen. Der noematische Satz/Sinn irgendeiner Bedeutungsintention untersteht einer bestimmten grammatisch-noematischen Kategorie.

(2) Andererseits entscheiden diese grammatisch-noematischen Kategorien, welche noematische Sätze/Sinne mit welchen noematischen Sätzen/Sinnen sich zu einem einheitlichen Satz/Sinn zusammenschließen können. Z.B. schreibt die grammatischnoematische Kategorie des Sinnes „und“ (in „Sokrates und Platon“) das folgende Gesetz vor: Als ein konjunktivisch-noematischer Sinn kann er mit zwei substantivischen Sätzen/Sinnen zu einem einheitlichen substantivischen Satz/Sinn verbunden werden, aber nicht mit zwei bindewörtlich-noematischen Sinnen (wie ,oder und aber"). In diesem Sinne sind die Komplikationsweisen der noematischen 
Sätze/Sinne (von Bedeutungsintentionen) durch gewisse von grammatischnoematischen Kategorien bestimmte Gesetze geregelt.

Wir können also im Rahmen der transzendentalen Phänomenologie, aufgrund des noematischen Bedeutungsbegriffs, die Grammatikalitätsthese der Bedeutungen in (die Grammatikalitätsthese der noematischen Sätze/Sinne von Bedeutungs-intentionen) wie folgt übersetzen:

(Die Grammatikalitätsthese der noematischen Sätze/Sinne von Bedeutungsintentionen)

Die noematischen Sätze/Sinne (=Bedeutungen) von Bedeutungsintentionen sind gewissen durch grammatisch-noematische Kategorien (=Bedeutungskategorien) bestimmten grammatischen Gesetzen unterworfen. Das bezeichnen wir als das Grammatikalitätsphänomen (bezüglich) der noematischen Sätze/Sinne von Bedeutungsintentionen.

Es ist zu bemerken, dass man im Rahmen der transzendentalen Phänomenologie nicht nur die noematische Seite, sondern auch die noetische Seite beachten soll. Deshalb haben wir in den Konstitutionsanalysen das Prinzip des noetisch-noematischen Parallelismus bewußt befolgt. Diesem Prinzip weiter folgend könnte man hier vermuten, dass es auf der noetischen Seite etwas Paralleles gibt, das dem Grammatikalitätsphänomen der noematischen Sätze/Sinne von Bedeutungsintentionen entspricht.

Im [§8] haben wir erwähnt, dass Husserl in $I V . L U$ die Unterscheidung von einfacher/zusammengesetzter Bedeutungsintention machte. Eine zusammengesetzte Bedeutungsintention als Gesamtakt besteht aus mehreren Bedeutungsintentionen als Teilakten. Dem Gesamtakt entspricht eine Gesamtbedeutung, und den Teilakten entspricht jeweils eine der Teilbedeutungen, die sich zu der Gesamtbedeutung zusammensetzen. Zum Beispiel entspricht der Bedeutungsintention, die durch den Ausdruck „weibliches Geschwister eines Elternteils“ kundgegeben wird, die Gesamtbedeutung [weibliches Geschwister eines Elternteils], und seinen Teilakten entsprechen jeweils die Bedeutungen [weiblich], [Geschwister], [ein] und [Elternteil]. Doch es genügt eine bloße Liste der Teilbedeutungen noch nicht, um die Gesamtbedeutung, die die aufgelisteten Teilbedeutungen bilden, zu repräsentieren. Denn die Teilbedeutungen können auf verschiedene Weisen kombiniert werden - man 
vergleiche die Bedeutungen [weibliches Geschwister eines Elternteils] und [weiblicher Elternteil eines Geschwisters]. Mit Bolzanos Terminologie: Die Liste gibt nur den Inhalt, aber nicht den Stoff der entsprechenden Bedeutungsintention an. ${ }^{411}$ Korrelativ entspricht der Art und Weise, in der die Gesamtbedeutung aus den Teilbedeutungen zusammengesetzt ist, eine bestimmte Art und Weise, in der der Gesamtakt aus den Teilakten besteht. ${ }^{412}$ In die Sprache der Ideen I übersetzt: Der Art und Weise, in der der noematische Gesamt-Satz/Sinn aus den noematischen Teil-Sätzen/Sinnen zusammengesetzt ist, entspricht eine bestimmte Art und Weise, in der die noetische Gesamt-Bedeutungsintention aus den noetischen Teil-Bedeutungsintentionen besteht. In Anlehnung an Beyer bezeichne ich dieses parallele Verhältnis zwischen den noematischen und noetischen Komplikationsweisen als strukturelle Isomorphie zwischen noematischen Sätzen/Sinnen und Bedeutungsintentionen. ${ }^{413}$

Nun haben wir festgestellt, dass die Komplikationsweisen, in denen sich noematische Sätze/Sinne (von Bedeutungsintentionen) zu einem umfassenderen Satz/Sinn zusammenschließen, nicht beliebig, sondern gewissen durch grammatischnoematische Kategorien (=Bedeutungskategorien) bestimmten grammatischen Gesetzen unterworfen sind. Korrelativ sind die Komplikationsweisen der Bedeutungsintentionen, die nach der strukturellen Isomorphie den der Sätze/Sinne entsprechen, auch durch solche grammatische Gesetze beschränkt. Das heißt, die Bedeutungsintentionen können sich nur in denjenigen Komplikationsweisen, die den grammatisch zulässigen Komplikationsweisen der noematischen Sätze/Sinne entsprechen, zusammenschließen.

Terminologisch können wir (die Grammatikalitätsthese der Noesis von Bedeutungsintentionenen) und die Rede des noetischen Grammatikalitätsphänomens von Bedeutungsintentionen einführen:

(Die Grammatikalitätsthese der Noesis von Bedeutungsintentionenen)

Die Noesen von Bedeutungsintentionen sind gewissen durch grammatischnoematische Kategorien (=Bedeutungskategorien) bestimmten grammatischen Gesetzen unterworfen. Das bezeichnen wir als das noetische Grammatikalitätsphänomen von Bedeutungsintentionen.

\footnotetext{
${ }^{411}$ Vgl. Bolzano, Wissenschaftslehre, §56; Christian Beyer, Von Bolzano zu Husserl, S.95-106.

${ }^{412}$ Christian Beyer, Von Bolzano zu Husserl, S.107, 128.

${ }^{413}$ Ebd.
} 


\section{§ 48. Reinterpretation der Grammatikalitätsthese der Gegenstände im Rahmen der noetisch-noematischen Analysen}

In [§§25-26] haben wir die Grammatikalitätsthese der Gegenstände eingeführt. Nach dieser These haben nur reinlogisch-grammatisch wohlgeformte Bedeutungen das Potential, entsprechende Gegenstände in der Wirklichkeit zu haben. Z.B. besteht in der Wirklichkeit gar kein Sachverhalt, der die Form „S ist und“ hat ${ }^{414}$. Zur Erinnerung:

(Die Grammatikalitätsthese der Gegenstände)

Die Gegenstände der geradehin urteilenden Einstellung sind gewissen durch

Bedeutungskategorien bestimmten grammatischen Gesetzen unterworfen. Das bezeichnen wir als das Grammatikalitätsphänomen (bezüglich) der

Gegenstände.

Geradehin urteilend untersucht man die Gegenstände innerhalb der natürlichen Einstellung. Das heißt, man hält die Existenz der betreffenden Gegenstände für selbstverständlich und versucht, sie zu erkennen und bestimmen ${ }^{415}$. In der phänomenologischen Einstellung ist aber die Existenz der Gegenstände ausgeklammert und man betrachtet sie in einer existenzneutralen Weise mittels des Begriffs des bestimmbaren X. Ich nehme an, dass sich die (Spezifikation des bestimmbaren X des Urteilsaktes) im [§43a] auf alle Bedeutungsintentionen mutatis mutandis übertragen lässt:

\section{(Spezifikation des bestimmbaren X der Bedeutungsintention)}

In jeder Bedeutungsintention wird ein $\mathrm{X}$ konstituiert, das entweder

(i) mit dem wirklichen Gegenstand, der so ist, wie er in der

Bedeutungsintention intendiert ist, zusammenfällt, oder

(ii) es verhielte sich so, wie in (i) beschrieben, wenn die Bedeutungsintention veridisch wäre.

(1) Einerseits sind noematische Sätze/Sinne (von Bedeutungsintentionen), wie oben erläutert, gewissen durch grammatisch-noematische Kategorien (=Bedeutungskategorien) bestimmten grammatischen Gesetzen unterworfen. Es gibt also kein Noema oder noematisches Feld (von Bedeutungsintentionen), das einen reinlogischungrammatischen Satz/Sinn als ein Moment enthält. (2) Andererseits ist das X (von

\footnotetext{
${ }^{414}$ Es ist sogar kein unmöglicher Sachverhalt wie „Das Viereck ist rund“.

${ }^{415}$ Vgl. [§23] der vorliegenden Arbeit.
} 
Bedeutungsintentionen) ein notwendiges Moment ${ }^{416}$ des Noema oder noematsichen Feldes. (1) und (2) zufolge kann ein bestimmbares X (von Bedeutungsintentionen) nur in einem Noema oder noematischen Feld, dessen noematsicher Satz/Sinn grammatisch wohlgeformt ist, konstituiert werden. In diesem Sinne können wir sagen, dass auch die Konstitution des bestimmbaren $\mathrm{X}$ von Bedeutungsintentionen gewissen durch Bedeutungskategorien bestimmten grammatischen Gesetzen unterworfen ist. Im Rahmen der transzendentalen Phänomenologie können wir also die Grammatikalitätsthese der Gegenstände wie folgt reinterpretieren:

(Die Grammatikalitätsthese des bestimmbaren X von Bedeutungsintentionen)

Die Konstitution des bestimmbaren X von Bedeutungsintentionen ist gewissen durch grammatisch-noematische Kategorien (=Bedeutungskategorien) bestimmten grammatischen Gesetzen unterworfen, wobei das bestimmbare $\mathrm{X}$ die (Spezifikation des bestimmbaren X der Bedeutungsintention) erfüllt.

Das bezeichnen wir als das Grammatikalitätsphänomen (bezüglich) des bestimmbaren Xvon Bedeutungsintentionen.

\section{§ 49. Der Zusammenhang der Grammatikalitätsthesen}

Mithilfe der noetisch-noematischen Analysen haben wir die beiden im I. Abschnitt aufgestellten Grammatikalitätsthesen, nämlich (die Grammatikalitätsthese der Bedeutungen) und (die Grammatikalitätsthese der Gegenstände) reinterpretiert: wir haben sie in der Sprache der transzendentalen Phänomenologie jeweils in (die Grammatikalitätsthese der noematischen Sätze/Sinne von Bedeutungsintentionen) und (die Grammatikalitätsthese des bestimmbaren X von Bedeutungsintentionen) übersetzt. Da sowohl der noematische Satz/Sinn als auch das bestimmbare X dem Noema oder noematischen Feld angehören, könnte man die beiden Thesen unter der folgenden These I zusammenfassen und die Rede des noematischen Grammatikalitätsphänomens von Bedeutungsintentionen einführen:

These I - (Die Grammatikalitätsthese des noematischen Feldes von Bedeutungsintentionen)

Sowohl die noematischen Sätze/Sinne als auch die Konstitution des

\footnotetext{
${ }^{416}$ Unterm funktionellen Gesichtspunkt (vgl. [§32d] und [§40] der vorliegenden Arbeit) konstituiert jede synthetische Einheit von intentionalen Akten ein gegenständliche Einheit, das Husserl unter dem Titel ,bestimmbares X“ bestimmt und durch die dynamischen Konstitutionsanalysen untersucht. In diesem Sinne ist das X ein notwendige Komponente des Noema.
} 
bestimmbaren $\mathrm{X}$ von Bedeutungsintentionen sind gewissen durch grammatisch-noematische Kategorien (=Bedeutungskategorien) bestimmten grammatischen Gesetzen unterworfen. Das bezeichnen wir als das noematische Grammatikalitätsphänomen von Bedeutungsintentionen.

Auf noetischer Seite haben wir im [\$47], dem Prinzip des noetisch-noematischen

Parallelismus folgend, (die Grammatikalitätsthese der Noesis von Bedeutungsintentionen) abgeleitet und somit die Rede des noetischen Grammatikalitätsphänomens von Bedeutungsintentionen eingeführt. Nennen wir sie These II.

Terminologisch können wir noch ein Stück weitergehen: Wir bezeichnen die Konjunktion von These I und These II als (die Grammatikalitätsthese von Bedeutungsintentionen), wobei die Gesamtheit des noematischen Grammatikalitätsphänomens und des noetischen Grammatikalitätsphänomens bezüglich der Bedeutungsintentionen als das noetisch-noematische Grammatikalitätsphänomen von Bedeutungsintentionen $\mathrm{zu}$ bezeichnen ist.

Insgesamt können wir den Zusammenhang der Grammatikalitätsthesen durch die folgende Graphik illustrieren:

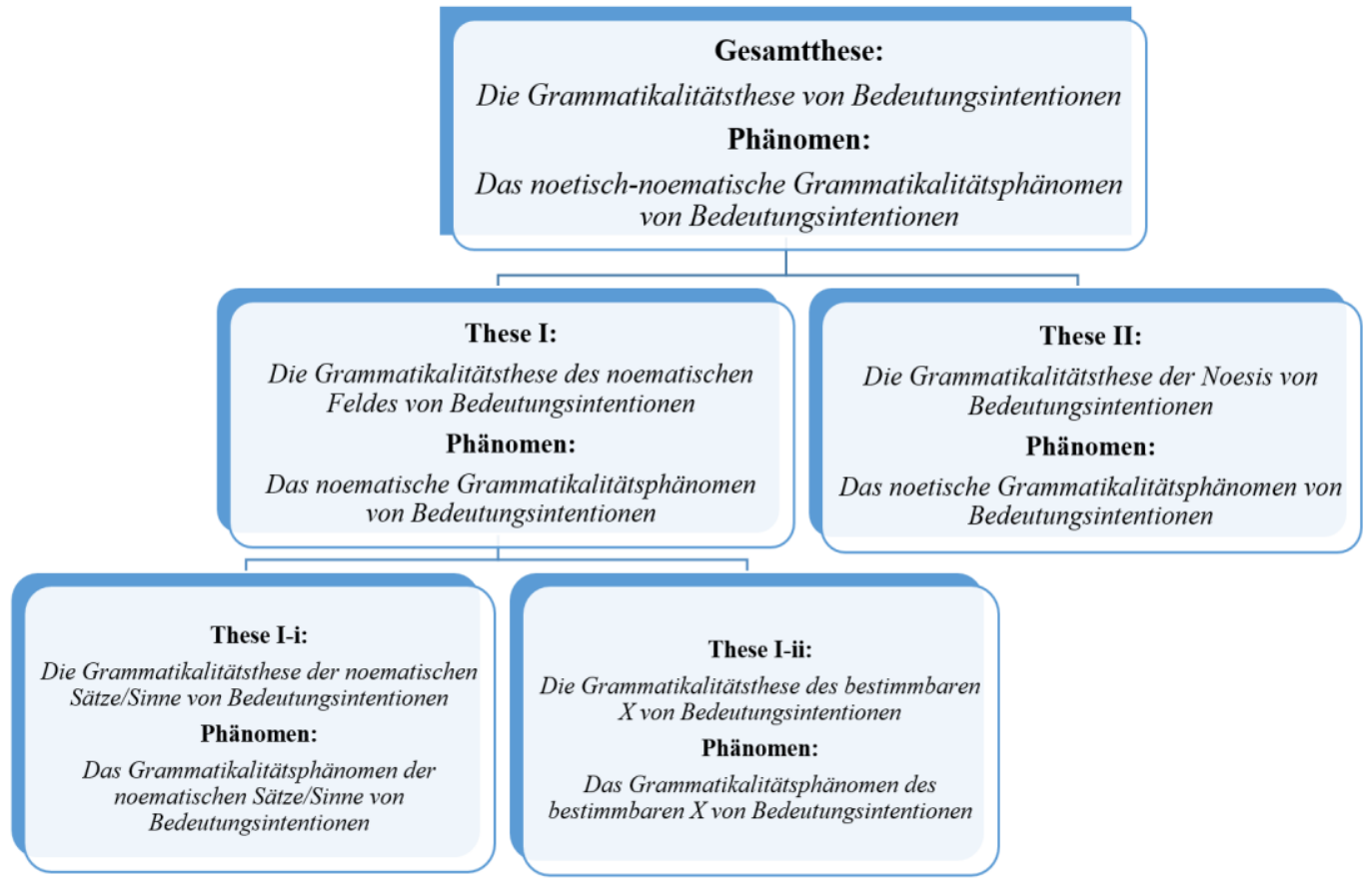

Wobei These I-i und These I-ii unsere transzendental-phänomenologische Reintepretation der beiden im I. Abschnitt aufgestellten Grammatikalitätsthesen darstellen. 


\section{§50. Zusammenfassung und Ausblick in den nächsten Abschnitt}

In diesem Abschnitt haben wir die Cartesianische Motivation für die phänomenologische Reduktion und die transzendentale Phänomenologie (im I. Kapitel) erklärt. Dann (in II. und III. Kapitel) betrieben wir punktuelle und dynamische Konstitutionsanalysen, in denen wir verschiedene noetische und noematische Momente kennengelernt haben. Insbesondere haben wir dabei den Begriff des noematischen Satzes/Sinnes und den des bestimmbaren $\mathrm{X}$ herausgearbeitet. Diese Begriffe sind für unsere transzendental-phänomenologische Reinterpretation der im I. Abschnitt aufgestellten Grammatikalitätsthesen unmittelbar relevant. Nach diesen begrifflichen Vorbereitungen sind wir schließlich in der Lage, die Grammatikalitätsthesen in die transzendentale Phänomenologie (im IV. Kapitel) zu transplantieren.

Bisher haben wir die Rede des noetisch-noematischen Grammatikalitätsphänomens auf den Bereich der Bedeutungsintentionen beschränkt. Allerdings bilden Bedeutungsintentionen nur eine Sonderklasse von objektivierenden Akten. Man möchte fragen, lässt sich die Rede des Grammatikalitätsphänomens auf die anderen objektivierenden Akte, nämlich die bedeutungerfüllenden Akte, erweitern? Darauf möchte ich im folgenden Abschnitt eine bejahende Antwort geben und sie rechtfertigen. 


\title{
III. ABSCHNITT: \\ DAS GRAMMATIKALITÄTSPHÄNOMEN DER BEDEUTUNGERFÜLLENDEN AKTE
}

\author{
I. KAPITEL \\ EINLEITUNG IN DIE URSPRUNGSANALYSEN VON ERFAHRUNG UND \\ URTEIL
}

\section{§51. Ursprungsanalyse des Urteils}

Husserls Enthüllungen der grammatischen Formen von bedeutungerfüllenden Akten bzw. Anschauungen finden sich in Erfahrung und Urteil, wo er versucht, die sogenannten Ursprungsanalysen des (evidenten) Urteils durchzuführen. Das führt dazu, dass die Analysen des Grammatikalitätsphänomens der bedeutungerfüllenden Akte bzw. Anschauungen im methodischen Kontext der Ursprungsanalysen durchgeführt werden. Um Husserls Beschreibungen des Grammatikalitätsphänomens der Anschauungen darzustellen, ist es unentbehrlich, zuerst den methodischen Kontext, nämlich die Idee von Ursprungsanalysen des Urteils einzuführen: Welche Überlegungen führen zu den Ursprungsanalysen? Welche Aufgaben haben die Ursprungsanalysen?

\section{$\S 52$. Von der formalen zur transzendentalen Logik}

In Formale und transzendentale Logik spricht Husserl von der sogenannten Doppelseitigkeit ${ }^{417}$ der Logik: Einerseits hat die Logik ihre objektive Thematik, d.h. sie hat die logischen Gebilde wie Begriffe, Urteile, Schlüsse zu untersuchen, andererseits sind diese Gebilde „Erzeugnisse“ der in der objektiven Thematik verborgenen konstitutiven Leistungen der Subjektivität. Diese Leistungen bilden die sogenannte subjektive Thematik der Logik. (Hua XVII, 38) In die Sprache der

\footnotetext{
${ }^{417}$ Hua XVII: Formale und transzendentale Logik, S.36. Man sollte die hier einschlägige Doppelseitigkeit von der Zweigliederung der formalen Logik, die wir oben im [§23] diskutierten, unterscheiden.
} 
transzendentalen Phänomenologie übersetzt: Der Logiker soll nicht nur die idealen Gebilde (Begriffe, Urteile, Schlüsse) einfach aufnehmen und studieren (obejektive Thematik), sondern auch noetisch-noematische Konstitutionsanalysen von ihnen, deren Grundideen schon im II. Abschnitt dargestellt wurden, durchführen; ${ }^{418}$ solche noetischnoematische Konstitutionsanalysen der idealen Gebilde (besonders der Urteile) machen eben die subjektive Thematik der Logik aus.

Husserl zufolge untersucht die historisch gewordene formale Logik (oder genauer, die formale Apophantik ${ }^{419}$ ) die idealen Urteile (hinsichtlich ihrer Formen ${ }^{420}$ ) und hat tatsächlich nur die objektive Thematik berücksichtigt; wir haben Husserls Charakterisierung dieser objektiven Thematik schon im III. Kapitel des I. Abschnitts ausführlich dargestellt. Was die subjektive Thematik anbelangt, hat der formale Logiker sie, mangels der Idee der transzendentalen Phänomenologie (a.a.O., 48, 238, 265), kaum bemerkt, geschweige denn thematisiert. Für Husserl ist die subjektive Thematik der formalen Logik tatsächlich bei manchen Philosophen (wie Descartes, Hume, Kant) verborgen geblieben. (a.a.O., §93, §100) Zum Beispiel hat Hume nach Husserls Interpretation zwar das Konstitutionsproblem bemerkt, aber nicht richtig verstanden, denn sein Sensualismus hat keine Ahnung von Intentionalität und phänomenologischer Reduktion.

Um die subjektive Thematik der Logik zu initiieren, muss man ,transzendentale Fragen“ (a.a.O., S.267) an die Logik stellen. Das sind Fragen, die um die Konstitution der Urteile als idealen Gebilde des Subjekts gehen. ${ }^{421}$ Man fragt zum Beispiel, ,,wie die Subjektivität in sich selbst rein aus Quellen ihrer Spontaneität Gebilde schaffen ${ }^{422}$ kann, die als ideale Objekte einer idealen ,Welt“ gelten können. ““223 Solche transzendentalen Fragen haben die Philosophen, die Husserl kritisiert, entweder ignoriert oder missdeutet.

\footnotetext{
${ }^{418}$ Dasselbe gilt auch für alle Wissenschaften. Vgl. ebd., S.43.

${ }^{419} \mathrm{Vgl}$. [\$22] der vorliegenden Arbeit.

${ }^{420}$ Für Husserl hat sie manchmal sogar die Idealität ihrer Forschungsgegenstände ignoriert. Vgl. ebd., S.45-50, 265.

${ }^{421}$ Hua XVII: Formale und transzendentale Logik, S.266.

${ }^{422}$ Der Begriff vom Schaffen gehört zum Kreis des Konstitutionsbegriffs, der, wie wir im [§32d] erörterten, ein operativer Begriff in Husserls Phänomenologie ist und in etwas metaphorischer Unbestimmtheit bleibt.

${ }^{423}$ Ebd., S.267. In Formale und transzendentale Logik hat Husserl auch andere Probleme, die die transzendentale Logik behandeln soll, erwähnt. Z.B. Probleme über die sogenannte idealisierende Voraussetzungen bei der formalen Logik. Darüber vgl. Dieter Lohmar, „Warum braucht die Logik eine Theorie der Erfahrung“, in: Phenomenology on Kant, German Idealism, Hermeneutics and Logic, hrsg. von O.K. Wiegand et al., Springer Science + Business Media, Dordrecht 2000, S.149ff.
} 
(a.a.O., §93, §100) Husserl zufolge lassen solche Fragen sich nur in Rahmen der transzendentalen Phänomenologie durch noetisch-noematische Konstitutionsanalysen beantworten. (a.a.O., 269) In diesem Sinne ist die Initiierung der logisch-subjektiven Thematik zugleich die Überwindung der ,,phänomenologischen Naivität“ der Logik, Husserl schreibt:

Sie $[=$ die Logik - Anm.d.Verf.] muß die phänomenologische Naivität überwinden, sie muß selbst nach errungener Anerkenntnis des Idealen mehr sein als eine bloß positive Wissenschaft von den logisch-mathematischen Idealitäten. Vielmehr in beständig zweiseitiger ... Forschung muß sie systematisch von den idealen Gebilden auf das sie phänomenologisch konstituierende Bewußtsein zurückgehen, diese Gebilde als wesensmäßige Leistungen der korrelativen Strukturen des leistenden Erkenntnislebens nach Sinn und Grenzen verständlich machen, und sie damit wie alle und jede Objektivität überhaupt dem weiteren, dem konkreten Zusammenhang der transzendentalen Subjektivität einordnen. (a.a.O., 270; meine Herv.)

Vom Gesichtspunkt der in Ideen I entwickelten Konzeption der transzendentalen Phänomenologie aus ist also die subjektive Thematik der Logik eigentlich eine Anwendung der Konstitutionsanalysen auf die konstituierten logisch-idealen Gebilde (Urteile) und die sie konstituierenden Bewußtseinstätigkeiten. Vor diesem Hintergrund kennzeichnet die subjektive Thematik der Logik eine besondere, auf die Konstitution der idealen Urteile bezogene Abteilung der allgemeinen transzendentalen Phänomenologie.

Außerdem spricht Husserl von dem Übergang von der subjektiven Thematik der Logik zur transzendentalen Phänomenologie überhaupt. Die transzendentale Frage, solange sie in der Logik an einer Art von Objekten (idealen Urteilen) gestellt wird, wird zur allgemeinen; denn die ideale Urteile sind nur eine besondere Art von Objekten und dementsprechend ist die auf sie bezogene transzendentale Frage nur eine besondere Version der transzendentalen Frage in allgemeinster Form - Es liegt nahe, von dieser speziell auf ideale Urteile bezogenen Fragestellung zu einer universalen Fragestellung über die Konstitution von Objektivität überhaupt durchzudringen (a.a.O., 271-273), die 
eben das allgemeinste Thema der transzendentalen Phänomenologie ausmacht. ${ }^{424}$ Die subjektiv orientierte Logik ,mündet letztlich in einer umfassenden phänomenologischen Analyse des Bewußtseins in der transzendentalen Reduktion “. 425

In diesem Zusammenhang mit der transzendentalen Phänomenologie wird die formale Logik, sobald sie die subjektive Thematik in Angriff nimmt, in eine transzendentale Logik verwandelt. ${ }^{426}$

\section{§53. Von der transzendentalen Logik zur Konsitutionsanalyse des evidenten \\ Urteils}

Die Hauptaufgabe der transzendentalen Logik ist zu untersuchen, wie ideale Urteile als noematische Komponenten in der Subjektivität durch ihre noetischen Leistungen konstituiert werden; allerdings sind nicht alle Urteile für den Logiker in gleichem Maße interessant.

(1) Für den Logiker treten die Urteile zunächst auf als prätendierte Erkenntnisse man urteilt nicht zum Spiel, sondern um Erkenntnisse über die ihn interessierenden Gegenstände zu erwerben. $(E U, 8,11,15)$ Urteile zielen darauf ab, Erkenntnisse über die entsprechenden Gegenstände zu sein, das bezeichnen wir als die Erkenntnisfunktion des Urteils. Eine notwendige Bedingung zur Erfüllung der Erkenntnisfunktion ist, dass das betreffende Urteil wahr ist. Aber offensichtlich erfüllt nicht jedes Urteil die Erkenntnisfunktion. Der Logiker kennt „,den Unterschied von Urteilen, die wirkliche Erkenntnis sind, denen Wahrheit zukommt, und solchen, die bloß vermeinte, bloß prätendierte Erkenntnis sind.“ (a.a.O., 7) Das Interesse, das der Logiker an den idealen Urteilen hat, ist für Husserl kein ,,bloßes Interesse an irgendwelchen Gebilden von bestimmter Form, sondern Interesse an Gebilden, die den Anspruch machen, Niederschlag von Erkenntnissen zu sein. “ (a.a.O.) Deshalb interessiert sich der Logiker vielmehr für die ,guten“ Urteile, nämlich diejenigen, die die Erkenntnisfunktion

\footnotetext{
${ }^{424}$ Hua XVII, S.271-273: ,Diese Frage, einmal an einer Art von Objekten gesehen, wird sofort zur allgemeinen: ist nicht alle und jede Objektivität, mit allem Sinn, in dem sie uns je gilt, in uns selbst zur Geltung kommende oder gekommene, und das mit dem Sinn, den wir uns selbst erworben haben?" Dazu vgl. auch Robert Sokolowski, The Formation of Husserl's Concept of Constitution, S.167-170.

${ }^{425}$ Dieter Lohmar, Edmund Husserls ,Formale und transzendentale Logik“, S.41.

${ }^{426}$ Ebd., S.35, 41, 113.
} 
erfüllen, und Husserl bezeichnet sie als Erkenntnisurteile; die ,schlechten“ Urteile, die es nicht tun, werden von Husserl als bloße Urteile bezeichnet und etwas als degenerierte Modifikation von Erkenntnisurteilen betrachtet, ${ }^{427}$ sie sind für den Logiker weniger interessant $^{428}$, denn Urteile zielen darauf, wie gesagt, Erkenntnisse über die entsprechenden Gegenstände zu sein. ${ }^{429}$

(2) Der Begriff von Erkenntnisurteil ist bei Husserl an manchen Stellen äquivalent mit dem Begriff des evidenten Urteils. ${ }^{430}$ Laut Husserl sind evidente Urteile diejenigen Urteile, die (i) entweder in einem evidenten Bewußtseinszusammenhang, wo der geurteilte Gegenstand durch Erfahrung selbstgegeben wird, ${ }^{431}$ (ii) oder in einem Schlussfolgerungszusammenhang, wo sie als Konklusion aus anderen evidenten Urteilen stammen, ${ }^{432}$ konstituiert werden. Nach Husserls Terminologie werden die Urteile, die (i) erfüllen, als unmittelbar evidente Urteile bezeichnet, während die (ii) erfüllenden Urteile als mittelbar evidente Urteile zu bezeichnen sind.

Aus (1) und (2) lässt sich der Schluss ziehen, dass sich die transzendentale Logik in erster Linie mit den evidenten Urteilen, die durch (2-i) oder (2-ii) charakterisiert und somit als unmittelbar evident oder mittelbar evident zu bezeichnen sind, beschäftigen soll. Mit einem anderen Wort: die primäre und zentrale Aufgabe der transzendentalen Logik ist, Konsitutionsanalysen von den unmittelbar und mittelbar evidenten Urteilen zu betreiben.

\footnotetext{
${ }^{427}$ Erfahrung und Urteil, S.15-16; Hua XVII: Formale und transzendentale Logik, S.218-219.

${ }^{428}$ Insgesamt: Die allgemeine Aufgabe der Logik ist, Urteile überhaupt zu untersuchen, gleichgültig, ob wahr oder falsch, konsistent oder inkonsistent, Erkenntnis oder nicht; jedoch interessiert sich der Logiker aus erkenntnistheoretischen Überlegungen mehr für die zentrale Aufgabe - Erkenntnisurteile zu untersuchen.

${ }^{429}$ Damit hängt auch zusammen, dass die höchste Schicht der formalen Apophantik, wie schon im [§20] erörtert, eben die Wahrheitslogik ist.

${ }^{430}$ Erfahrung und Urteil, S.11, 14, 15; Hua XVII: Formale und transzendentale Logik, S.213. Husserl scheint die Begriffe wahres Urteil, evidentes Urteil und Erkenntnisurteil ohne weiteres als umfangsgleich zu betrachten. Diese Umfangsäquivalenz ist problematisch und umstritten. Künne 1992 argumentiert, dass der Umfang des wahren Urteils dem des evidenten Urteils nicht gleich ist. Gettiers berühmter Aufsatz (1963) zeigt, dass evidente und wahre Urteile nicht Erkenntnisurteile sein müssen. Aber ich halte es für sinnvoll und gebe Husserl Recht, bei der Untersuchung von Erkenntnisurteilen mit evidenten Urteil anzufangen.

431 Erfahrung und Urteil, S.12. Selbstgegebenheit ist eben der noematische Anschauungscharakter von Gegenwärtigung oder „originär“, der oben im [§35] erläutert wurde.

432 Ebd., S. 17.
} 


\section{§54. Von der Konstitutionsanalyse des evidenten Urteils zur Ursprungsanalyse des Urteils}

Allerdings sind auch nicht alle evidenten Urteile für die transzendentale Logik in gleichem Maße interessant. Husserl legt deutlich mehr Gewicht auf die unmittelbar evidenten Urteile als die mittelbar evidenten, denn er ist der Meinung, dass die unmittelbar evidenten Urteile in der „Erkenntnisgenesis“ ursprünglicher sind als die mittelbar evidenten: die mittelbar-evidenten Urteile bauen auf den unmittelbarevidenten Urteilen durch Schlussfolgerungen auf. ${ }^{433}$ Deshalb sind unmittelbar-evidente Urteile für Husserl „Urteile des (erkenntnisgenetischen) Ursprungs“ 434 oder „ursprünglichste Urteile“ ${ }^{435}$; und die Analysen, die die Konstitution von unmittelbar-evidenten Urteilen studieren, werden von Husserl als Ursprungsanalysen des Urteils (oder Ursprungsklärung des Urteils) ${ }^{436}$ bezeichnet. Wegen der Sonderstellung der unmittelbar-evidenten Urteile innerhalb der Erkenntnisgenesis gilt es der transzendentalen Logik als die allererste und zentralste Aufgabe, die Ursprungsanalysen des Urteils zu betreiben.

Husserl vertritt die etwas natürliche und naheliegende Meinung, dass die ursprünglichsten Urteile der Erkenntnisgenesis Urteile über Individuen (Individualurteile) sind. ${ }^{437}$ Damit die betreffenden Individualurteile evident sind, müssen ihre Gegenstände-worüber, in der Sprache von Ideen I, im noematischen Anschauungscharakter ${ }^{438}$ des ,gegenwärtig“ oder „originär“ selbstgegeben sein. Husserl schreibt:

Sollen die Urteile evidente Urteile sein, so genügt es nicht, daß irgendwie

\footnotetext{
${ }^{433}$ Ebd., S.18:,,Bevor wir die Formen mittelbarer Erkenntnisse und Erkenntnisbegründungen studieren können, müssen wir also zunächst die der unmittelbaren, der schlichtesten Erkenntnisse, bezw. Erkenntnisaktivitäten studieren. Sie sind in der Erkenntnisgenesis, in der Formbildung der Erkenntniserzeugung die ursprünglichsten. Das heißt, es sind Leistungen, die schon vollzogen sein müssen, wenn die mittelbaren möglich werden sollen. “

${ }^{434}$ Hua XVII: Formale und transzendentale Logik, S.219.

${ }^{435}$ Erfahrung und Urteil, S. 16.

${ }^{436}$ Ebd., S.50-51.

${ }^{437}$ Vgl. Erfahrung und Urteil, S.20; Hua XVII, S.212. Husserls Grund dafür lautet: Die ursprünglichsten Urteile müssen syntaktisch am einfachsten sein: z.B. darf das „S“ (,dass der Himmel blau ist“) in der Form „S ist p“ (,dass der Himmel blau ist, ist erfreulich“) auf keine tiefere syntaktische Sturktur wie „S' ist q“ (,,der Himmel ist blau“) zurückweisen; Urteile, die syntaktisch komplizierter sind (wie „,dass der Himmel blau ist, ist erfreulich“), sind in der Erkenntnisgenesis von den syntaktisch-einfachen abgeleitet (FTL, S.215; EU, S.19); nur Urteile, deren Gegenständeworüber Individuen sind, können die syntaktische Einfachheit erfüllen.

${ }^{438}$ Vgl. [§35] der vorliegenden Arbeit.
} 
irgendwelche Gegenstände vorgegeben sind, und daß sich das Urteilen auf sie richtet ... Vielmehr stellt das Gelingen der Erkenntnisleistung auch seine Anforderungen an die Weise der Vorgegebenheit der Gegenstände selbst in inhaltlicher Beziehung. Sie müssen ihrerseits so vorgegeben sein, daß ihre Gegebenheit von sich aus Erkenntnis und das heißt evidentes Urteilen möglich macht. Sie müssen selbst evident, als sie selbst gegeben sein. ${ }^{439}$

Ich nehme an, dass Husserl im Zitat bei der Rede von „evidenten Urteilen“, wie es in Erfahrung und Urteil oft der Fall ist, vor allem die ursprünglichsten Urteile im Auge hat, so ist dem Zitat zu entnehmen, dass die ursprünglichsten Urteile nur aufgrund der Selbstgegebenheit der betreffenden individuellen Gegenstände-worüber konstituiert werden können. Die Hauptaufgabe der Ursprungsanalyse stellt sich heraus: Sie ist die Aufgabe, zu beschreiben, wie die ursprünglichsten Urteile aufgrund der Selbstgegebenheit der (individuellen) Gegenstände konstituiert werden.

\section{$\S 55$. Von Urteil zu Erfahrung}

Husserl zufolge wird die Selbstgegebenheit der individuellen Gegenstände, auf deren Grund die ursprünglichsten Urteile konstituiert werden, durch Erfahrung (paradigmatisch: äußere Wahrnehmung) geliefert. ${ }^{440}$ Husserl:

Daher sind die an sich ersten Urteile als Urteile mit individuellen Substraten, Urteile über Individuelles, die Erfahrungsurteile. Die evidente Gegebenheit von individuellen Gegenständen der Erfahrung geht ihnen voran, d.i. ihre vorprädikative Gegebenheit. $(E U, 21)$

Diese dem ursprünglichsten Urteil (qua idealem Urteil) „,vorangehende“ Erfahrung (qua intentionalen Akt) nennt Husserl auch gegenständliche Evidenz, im Vergleich dazu werden die evidenten Urteilsakte, in denen ein ursprünglichstes Urteil (qua ideales Urteil) konstituiert werden, als Urteilsevidenz bezeichnet. (a.a.O.,13-14) Husserls Beispiel von gegenständlicher Evidenz ist ein Wahrnehmungsakt, in dem eine gelbe Kupferschale gegeben wird, dementsprechend ist der Urteilsakt „die Kupferschale ist

\footnotetext{
${ }^{439}$ Erfahrung und Urteil, S.11.

${ }^{440}$ Erfahrung und Urteil, S.21; Hua XVII: Formale und transzendentale Logik, S.213.
} 
gelb“, der aufgrund der Wahrnehmung vollzogen wird, Urteilsevidenz hinsichtlich der Kupferschale. Das Verhältnis zwischen den beiden Evidenzen (oder evidenten Akten) beschreibt Husserl wie folgt:

Gegenständliche Evidenz ist die ursprünglichere, weil die Urteilsevidenz erst ermöglichende, und die Ursprungsklärung des prädikativen Urteils muß verfolgen, wie sich auf gegenständliche Evidenz das evidente prädikative Urteilen aufbaut; und das zunächst für die primitivsten Leistungen prädikativen Urteilens. (a.a.O.)

An einer anderen Stelle spricht Husserl vom Fundierungsverhältnis von Urteilen und Erfahrung: Unter dem Gesichtspunkt der Erkenntnisgenesis ist ein evidenter Urteilsakt, in dem ein ursprünglichstes Urteil (qua ideales) auf noematischer Seite konstituiert wird, fundiert auf einem Erfahrungsakt (oder Erfahrungsakten). ${ }^{441}$ Dieses Fundierungsverhältnis schließt die Erfahrung und den Urteilsakt zu einer Erfüllungseinheit bzw. Erkenntniseinheit zusammen, wovon früher ${ }^{442}$ schon gesprochen wurde: Der Urteilsakt, der typischerweise als Bedeutungsintention fungiert, deckt sich mit einer Erfahrung (z.B. Wahrnehmung), sodass ein einheitlicher Akt des Erkennens (= die Erkenntniseinheit) vollzogen wird. ${ }^{443}$

Aus diesem Grund muss die Ursprungsanalyse des Urteils, die die Konstitution von ursprünglichsten Urteilen studiert, auch die dabei fundierende Erfahrung in Betracht ziehen. Husserl sieht sogar die Untersuchung der Erfahrung als den Ausgangspunkt der Ursprungsanalyse:

Die Evidenz der Erfahrung wäre sonach die von uns gesuchte letztursprüngliche Evidenz und damit der Ausgangspunkt der Ursprungsklärung des prädikativen Urteils. Theorie der vorprädikativen Erfahrung, eben derjenigen, die die ursprünglichsten Substrate [= individuelle

\footnotetext{
${ }^{441}$ Erfahrung und Urteil, S.12: „Aber ein evidentes prädikatives Urteil über ihn [= den Gegenstand-worüber] ist nicht möglich, ohne daß er selbst evident gegeben ist. Das hat zunächst für Urteile auf Grund der Erfahrung nichts Befremdliches, ja hier scheint mit dem Hinweis auf die Fundierung der prädikativen Evidenz in einer vorprädikativen nur eine Selbstverständlichkeit ausgesprochen zu sein." Näheres über den Begriff von ,vorprädikativ“" vgl. unten [§57].

${ }^{442} \mathrm{Vgl}$. [§5] und [\$41] der vorliegenden Arbeit.

${ }^{443}$ Hua XIX/2: Logische Untersuchungen, S.558-571.
} 
Gegenstände - Anm.d.Verf.] ${ }^{444}$ in gegenständlicher Evidenz vorgibt, ist das an sich erste Stück der phänomenologischen Urteilstheorie. ${ }^{445}$

Mit einem Wort: Die Ursprungsanalyse des Urteils enthält als erste Aufgabe eine Analyse über Erfahrung. ${ }^{446}$

\section{§ 56. Zusammenfassung des Kapitels}

Bis jetzt wird es verständlich, welche Überlegungen Husserl von einer relativ allgemeinen Konzeption der transzendentalen Logik (im [§52]) über eine differenziertere (im [§53]) bis zur Konzeption der Ursprungsanalyse des Urteils (im [§54]) und darunter letztlich zur Konzeption der Erfahrungsanalyse geführt haben. Das oben Ausgeführte lässt sich auch durch das folgende Zitat zusammenfassen:

Danach ist unter Gesichtspunkten dieser Genesis die an sich erste Urteilstheorie die Theorie der evidenten Urteile, und das an sich erste in einer Theorie der evidenten Urteile (und damit in einer Urteilstheorie überhaupt) ist die genetische Rückführung der prädikativen Evidenz auf die nichtprädikative Evidenz, die da Erfahrung heißt. ${ }^{447}$

In Formale und transzendentale Logik, wo die Konzeption der Ursprungsanalyse des Urteils und darunter die der Erfahrungsanalyse eingeführt sind, hat Husserl die konzipierten Analysen noch nicht ausführlich vollzogen. Er verweist den Leser auf Erfahrung und Urteil, wo die betreffenden Analysen durchgeführt werden. ${ }^{448}$ Doch in Formale und transzendentale Logik verrät er auch besondere Details:

\footnotetext{
${ }^{444}$ Aus dem Kontext geht hervor, dass Husserl hier mit „ursprünglichsten Substraten“ eben individuelle Gegenstände meint. In Erfahrung und Urteil ist der Term ",Substrat“ (und somit der Term ,individuelle Gegenstände“) vieldeutig und Husserl diskutiert diese Ambiguität ausführlich im [§29]. Der Einfachheit halber wird hier von den verschiedenen, miteinander zusammenhängenden Sinnen von „Substrat“ abgesehen. Hier genügt es, darauf hinzuweisen, dass Husserl im Textzusammenhang mit „ursprünglichen Substraten“ und „,individuellen Gegenständen“" vor allem die Naturobjekte (im Gegensatz zu Kulturobjekten) im Auge hat. Dazu vgl. Erfahrung und Urteil, S.54ff, S.158ff.

${ }^{445}$ Erfahrung und Urteil, S.21.

${ }^{446}$ Laurence Bonjour () sieht Husserls Begriff von vorprädikativer Erfahrung als eine Art Fundamentalismus: Unter dem Gesichtspunkt der Epistemologie ist Husserls vorprädikative Erfahrung, so sagt Bonjour, ein semi-kognitiver mentaler Zustand, der Überzeugungen rechtfertigt, aber selber keine Rechfertigung braucht. Bonjours Einwand: Das Postulat von semi-kognitiven Zuständen als ein Versuch, den unendlichen Regress von Begründung zu vermeiden, zu ad hoc ist. Hierbei stellt sich aber zuerst die Frage, ist Husserls Bild der Erkenntnisgenesis, wie Laurence Bonjour denkt, wirklich ein fundamentalistische Bild? Darüber gibt es eine Kontroverse (Vgl. Beyer 2013).

${ }^{447}$ Hua XVII: Formale und transzendentale Logik, S.217.

${ }^{448}$ Ebd., S.220.
} 
Besonders hervorgehoben sei nur, daß schon diese fundierende Erfahrung ihre Weise der syntaktischen Leistungen hat, die aber noch frei sind von all den begrifflichen und grammatischen Formungen, die das Kategoriale im Sinne des prädikativen Urteils und der Aussage charakterisieren. ${ }^{449}$

Diese syntaktischen (genauer: vor-syntaktischen) Leistungen der Erfahrung werden uns in den folgenden Kapiteln beschäftigen.

${ }^{449}$ Ebd. 


\section{KAPITEL \\ SYNTAKTISCHE LEISTUNGEN DER ERFAHRUNG}

\section{§57. Die Stufen des Erkenntnisstrebens}

Ein Begriff, der durch den ganzen Text von Erfahrung und Urteil hindurchgeht, ist das Begriffsduo von Erkenntnisinteresse und Erkenntnisstreben. Solange man theoretisches Interesse an einem gegebenen Gegenstand (= Erkenntnisinteresse) hat, hat man die Tendenz, den Gegenstand aufmerksam zu erfassen, seine Details zu betrachten und ihn letztlich prädikativ zu bestimmen (= Erkenntnisstreben). ${ }^{450} \mathrm{In}$ seinem Umgang mit der Welt hat man nicht nur theoretisches, sondern auch praktisches Interesse; in vielen Fällen ist das theoretische Interesse einem praktischen Zweck untergeordnet $^{451}$ - Sherlock Holmes, zum Beispiel, betreibt eine sehr abseitige wissenschaftliche Untersuchung von Tabakaschen nicht aus rein-theoretischer Neugier, sondern für das praktische Ziel, Kriminalfälle zu lösen. Um sich auf das Erkenntnisinteresse und Erkenntnisstreben zu konzentrieren, macht Husserl „die Fiktion eines Subjektes“, das die Welt nur theoretisch betrachtet und zu keiner praktischen Handlung veranlaßt wird. ${ }^{452}$

Husserl zufolge verläuft das Erkenntnisstreben durch zwei Hauptstufen: die vorprädikative Stufe (= die Stufe der Rezeptivität oder die rezeptive Stufe) und die prädikative Stufe (= die Stufe der Spontaneität oder die spontane Stufe). Beide Hauptstufen geliedern sich wiederum in ihre jeweiligen Substufen. Wir werden sehen, dass im Verlauf von den niederen zu den höheren Stufen die noematischen Gebilde allmählich mit immer größerer syntaktischer Komplexität erwachsen; dabei wird es auch ersichtlich, dass schon auf der vorprädikativen Stufe gewisse vor-grammatische Formbildungen als Vorgänger von Bedeutungskategorien konstituiert werden. Deshalb ist es nicht ohne Nutzen, Husserls Geschichte des gestuften Verlaufes vom Erkenntnisstreben und damit auch die mehrstufige, genetische Konstitutionsgeschichte

\footnotetext{
${ }^{450}$ Erfahrung und Urteil, S.11, 86-87, 91-93, 235-239,

${ }^{451}$ Ebd., S.68-69, 238-239.

$452 \mathrm{Ebd}$.
} 
der grammatischen Formen im folgenden Paragraphen zu erzählen, wo wir die Rede des Grammatikalitätsphänomens auf bedeutungerfüllende Akte erweitern wollen. Aber zunächst brauchen wir im folgenden Paragraph eine noch „frühere“ Stufe vor dem Erkenntnisstreben, nämlich die der Passivität, kurz zu betrachten.

\section{§ 58. Das passiv konstituierte Erfahrungsfeld und die affizierende Kraft auf das Subjekt}

Ein erkennendes Subjekt, das sich für einen in der Erfahrung (z.B. Wahrnehmung) gegebenen Gegenstand interessiert, beginnt das Erkenntnisstreben mit einer thematischen Zuwendung zu dem Gegenstand. Aber bevor das Subjekt den Gegenstand thematisiert, muss er zuerst schon in einem Erfahrungsfeld auftreten, sich von anderen Gegenständen des Feldes abheben und die Aufmerksamkeit des Subjekts anziehen. Ein Beispiel von Erfahrungsfeld ist die Sichtweite, in der ich jetzt alles, was sich in meinem Zimmer befindet (Tisch, Bücher, Regale usw.), sehen kann - das ist die Umgebung innerhalb des Zimmers. Solche Erfahrungsfelder bezeichnet Husserl als Feld der Vorgegebenheit:

Das Wahrnehmen, die wahrnehmende Zuwendung zu einzelnen Gegenständen, ihre Betrachtung und Explikation ${ }^{453}$, ist bereits eine aktive Leistung des Ich. Als solche setzt sie voraus, daß uns schon etwas vorgegeben ist, dem wir uns in der Wahrnehmung zuwenden können. Und vorgegeben sind nicht bloß einzelne Objekte, isoliert für sich, sondern es ist immer ein Feld der Vorgegebenheit. (EU, S.74)

Die Konstitution dieses Feldes lässt sich auch im Rahmen der transzendentalen Phänomenologie durch ,,sehr umfassende Analysen“ (a.a.O.) untersuchen, sie ist aber nicht das Thema von Erfahrung und Urteil. Für Husserl genügt es schon, folgende kurze Andeutungen zu erwähnen.

(1) Die Gegebenheiten innerhalb eines Erfahrungsfeldes befinden sich in einem Zusammenhang von Koexistenz (Zugleichsein) und Sukzession (Nacheinandersein)

\footnotetext{
${ }^{453}$ Zum Begriff der Explikation vgl. unten [§59] der vorliegenden Arbeit.
} 
innerhalb der Zeit. Mit Husserls eigenen Worten:

So haben also alle wahrgenommenen und je wahrnehmbaren Individuen die gemeine Form der Zeit. Sie ist die erste und Grundform, die Form aller Formen, die Voraussetzung aller sonst Einheit stiftenden Verbundenheiten. „Form“ besagt hier aber von vornherein den notwendig allem anderen in der Möglichkeit einer anschaulichen Einheit vorausgehenden Charakter. Die Zeitlichkeit als Dauer, als Koexistenz, als Folge ist die notwendige Form aller einheitlich anschaulichen Gegenstände und sofern ihre Anschauungsform (Form der konkret individuellen Angeschautheiten). (a.a.O., 191)

Deshalb bildet die Analyse über die Konstitution der Zeitform von Erfahrungsgegenständen ein Grundstück der „,sehr umfassenden Analysen“, die die Konstitution des Erfahrungsfeldes untersuchen. Die betreffenden Analysen über Zeitkonstitution führen zu Husserls Lehre des Zeitbewußtseins. ${ }^{454}$

(2) Die Gegebenheiten innerhalb eines Feldes der äußeren Erfahrung (z.B. der äußeren Wahrnehmung) sind nicht nur zeitlich, sondern auch räumlich organisiert: Sie haben bestimmte räumliche Gestalten, stehen in gewissen räumlichen Verhältnissen zueinander. Deshalb bildet die Analyse über die Konstituition der Raumform von Erfahrungsgegenständen ein anderes Grundstück der „sehr umfassenden Analysen“, die die Konstitution des Erfahrungsfeldes untersuchen. Die betreffenden Analysen über Konstitution von räumlichen Dingen führen letztlich zu Husserls Lehre über den bei äußerer Wahrnehmung als Wahrnehmungsorgan fungierenden Leib und die dabei wahrgenommenen räumlichen Dinge. ${ }^{455}$

(3) Die Gegebenheiten innerhalb eines Erfahrungsfeldes sind voneinander abgehobene Einheiten, denen das Subjekt sich zuwenden kann. Eine einzelne Gegebenheit im Erfahrungsfeld ist abgehoben durch Kontrast gegen die anderen. Zum

\footnotetext{
${ }^{454}$ In Erfahrung und Urteil wird darüber nur an vereinzelten Stellen (S.76, 180-184,190-194, 38, 303-309) diskutiert. Zur kurzen Erörterung des Zeitbewußtseins vgl. John Barnett Broughs Translator's Introduction in seiner Übersetzung von Hua IV. (On the Phenomenology of the Consciousness of Internal Time, Kluwer Academic Publishers, Dordrecht/Boston/London 1996, S.XI-LVII).

${ }^{455}$ Zur vorläufigen Verständigung über die Funktion des Leibes bei äußerer Wahrnehmung (Stichwort: Kinästhese) vgl. Hua XI: Analysen zur passiven Synthesis, S.13ff; Erfahrung und Urteil, S.71-72; dazu vgl. auch David Bell, Husserl, S.213-214.
} 
Beipiel fallen rote Flecken auf einem weißen Hintergrund auf, weil sie gegen die weiße Fläche kontrastieren und sich durch relative Ähnlichkeit ${ }^{456}$ „,verschmelzen“; und die roten Flecken heben sich wieder voneinander ab, weil sie sich durch die Raumlage voneinander trennen. Dabei herrschen die assoziativen Synthesen, die Ähnliches mit Ähnlichem zusammenbringen und von Unähnlichem trennen. Noematisch ist also das Erfahrungsfeld durch Homogeneität (Ähnlichkeit) und Heterogeneität (Unähnlichkeit) der Gegebenheiten organisiert, noetisch ist die Assoziationskraft des Bewußtseins dafür zuständig. (a.a.O., 74-79) Deshalb bildet die Analyse über die Konstituition des Abgehobenseins von Erfahrungsgegenständen noch ein Stück der „sehr umfassenden Analysen“, die die Konstitution des Erfahrungsfeldes untersuchen. Das führt zu Husserls Lehre über das Assoziationsphänomen des Bewußtseins.

Husserl zufolge können die in (1), (2) und (3) erwähnten konstitutiven Leistungen ${ }^{457}$ ohne Einsetzung des Erkenntnisstrebens vollzogen werden. Da das Erkenntnisstreben den Charakter der Aktivität (vom Subjekt) ${ }^{458}$ hat, fasst Husserl die das Erfahrungsfeld konstituierenden Leistungen noetisch unter dem Titel von ,ursprünglicher Passivität“ oder ,purer Passivität“ zusammen ${ }^{459}$, noematisch bezeichnet er das dabei konstituierte Feld auch als Feld passiver Vorgegebenheiten ${ }^{460}$. Solange die Vorgegebenheiten innerhalb des Feldes konstituiert sind, entfalten sie affektive Tendenz auf das Subjekt (genauer: das Ich) hin, anders gewendet: sie „haben ihre affektive Kraft, üben auf das Ich einen Reiz aus zur Zuwendung“. (a.a.O., 80) Andererseits hat das Subjekt die Tendenz, dem Reiz nachzugeben und sich dem affizierenden Gegenstand zuzuwenden. Husserl:

\footnotetext{
${ }^{456}$ Im Kontrast zu dem Hintergrund.

${ }^{457}$ Erfahrung und Urteil, S.24, 60-64.

${ }^{458}$ Ebd., S.74: „Das Wahrnehmen, die wahrnehmende Zuwendung zu einzelnen Gegenständen, ihre Betrachtung und Explikation, ist bereits eine aktive Leistung des Ich. Als solche setzt sie voraus, daß uns schon etwas vorgegeben ist, dem wir uns in der Wahrnehmung zuwenden können.“ Ebd., S.300: „Die Gegenstände der Rezeptivität sind vorgegeben in ursprünglicher Passivität mit ihren Strukturen der Assoziation, Affektion usw. Ihr Erfassen ist eine niedere Stufe der Aktivität, bloßes Rezipieren des ursprünglich passiv vorkonstituierten Sinnes. “

${ }^{459}$ Ebd., S.73, 83, 300. Davon ist die sogenannte „Passivität in der Aktivität“ zu unterscheiden. Vgl. ebd., S.119.

${ }^{460}$ Darüber mehr vgl. $E U, \S \S 7,12,13,15-17$. Im Vergleich zu den paradigmatischen Fällen, wo das Subjekt aktiv den Gegenstand betrachtet, expliziert und sogar urteilsmäßig (davon unten noch zu sprechen) bestimmt, scheint das passiv konstituierte Wahrnehmungsfeld zu niederstufig, um als ,noematisch“ bezeichnet zu werden. Aber auch in dieser Stufe werden doch einige Leistungen für die Konstitutionsleistungen der höheren Stufen (wie Explikation und Urteil, davon unten noch zu sprechen) organisiert und vorbereitet - ein noch zu artikulierendes Erfahrungsfeld. Terminologisch möchte ich diesen marginalen Fall als noematisch bezeichnen, in dem mininalen Sinn: nicht paradigmatisch noematisch, aber nicht nichts.
} 
Es hebt sich durch seine Intensität heraus aus einer Mehrheit von Affizierendem. Z.B. ist in der sinnlichen Sphäre ein Ton, ein Geräusch, eine Farbe mehr oder minder aufdringlich. Sie liegen im Wahrnehmungsfelde und heben sich aus ihm heraus, üben, noch nicht erfaßt, auf das Ich einen stärkeren oder schwächeren Reiz.

Ein Neues tritt ein, wenn das Ich dem Reiz folgt. Der Reiz des intentionalen Objektes in seiner Richtung auf das Ich zieht dieses mit stärkerem oder minder starkem Zuge an, und das Ich gibt nach ... das Ich wendet sich dem Objekt zu. (a.a.O., 80-81)

Im Zitat spricht Husserl mehrmals von dem „Ich“. Genauer gesprochen ist das Ich in diesem Textzusammenhang die Abteilung des Subjekts, die für die aktiven Leistungen des Erkenntnisstrebens (im Gegensatz zu den passiv-konstitutiven Leistungen des Subjekts) zuständig ist. ${ }^{461}$ Sobald das Erkenntnisstreben mit der gegenständlichen Zuwendung bei einem passiv vorgegebenen Gegenstand beginnt, fängt die aktive IchAbteilung an, zu funktionieren. Deshalb fasst Husserl die aktiven Leistungen des Erkenntnisstrebens (d.i. Zuwendung, Explikation, Prädikation, darüber mehr unten) auch unter dem Titel ,aktive Leistung des Ich“ oder „,ichliche Aktivität“ (a.a.O., 74, $75,86)$ zusammen.

Das Subjekt (genauer: das Ich) entscheidet ${ }^{462}$, ob es sich einem affizierenden Gegenstand aufmerksam zuwendet; und sobald es sich dem Gegenstand zuwendet, ist es schon in die erste Hauptstufe des Erkenntnisstrebens, d.i. die vorprädikative bzw. rezeptive Stufe, eingetreten. Die Rede von Rezeptivität scheint den Aktivitätscharakter auszuschließen, aber es ist zu bemerken, dass für Husserl die Rezeptivität auch der Aktivität, obschon nur als die unterste Stufe, angehört ${ }^{463}$; der Gegensatz zu Aktivität ist

\footnotetext{
${ }^{461}$ Der Einfachheit halber wird hier von einer ausführlichen Diskussion über Husserls Ich-Begriff abgesehen. Hier ist nur zu ergänzen, dass das (transzendentale) Ich in anderen Textzusammenhängen auch als ein identischer Pol, der als „Bewußtseinstätiges und Affiziertes in allen Bewußtseinserlebnissen lebt“ und dadurch habituelle Bestimmheiten als personalen Charakter erwirbt. Darüber vgl. Hua I, S.100ff.

${ }^{462}$ Husserl erwähnt auch die Fälle, wo die Zuwendung zum Gegenstand scheint keine Entscheidung des Ich zu sein. Z.B. kann ich bei der Beschäftigung mit einer wissenschaftlichen Arbeit durch einen Lärm von der Straße gestört sein: meine Zuwendung wird dann von der wissenschaftlichen Arbeit auf den Lärm, vielleicht für wenige Sekunden, abgelenkt. (Erfahrung und Urteil, S.92) Aber auch im Fall solcher verfärbten Zuwendung ist das Ich in der Lage, zu entscheiden, ob es die Aufmerksamkeit bei dem ablenkenden Gegenstand dauernd bleiben lässt oder nicht. In diesem Sinne befindet sich die verfärbte Zuwendung auch in Reichweite der Ich-Entscheidung.

${ }^{463} E U$, S.83: ,Sofern das Ich in der Zuwendung aufnimmt, was ihm durch die affizierenden Reize vorgegeben ist,
} 
eher die pure Passivität des Subjekts, die für die konstitutiven Leistungen der passiven Vorgegebenheiten zuständig ist. ${ }^{464}$

Die Erfahrung (z.B. Wahrnehmung) umfasst sowohl die Stufe der Passivität als auch die der Rezeptivität: einerseits konstituiert die passive Stufe der Erfahrung das Feld der Vorgegebenheiten, andererseits fasst die aktiv-rezeptive Stufe eine Vorgegebenheit heraus und dann betrachtet sie in Details. ${ }^{465}$ Die weiteren Analysen vorwegnehmend können wir die Verhältnisse zwischen den Begriffsgruppen von Passivität/Aktivität, Passivität/Rezeptivität/Spontaneität sowie Erfahrung/Urteil durch das folgende Diagramm illustrieren:

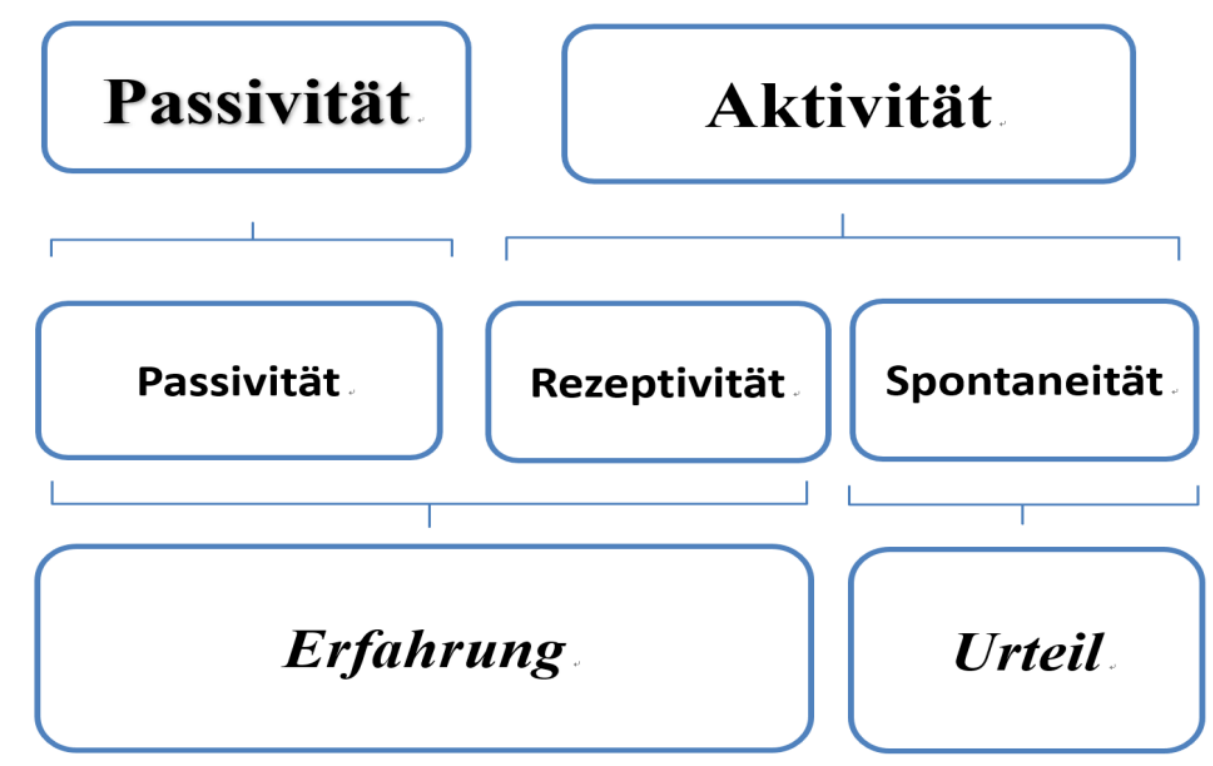

Im nächsten Schritt wollen wir die erste Hauptstufe des Erkenntnisstrebens, nämlich die der Rezeptivität, betrachten. ${ }^{466}$

können wir hier von der Rezeptivität des Ich sprechen. Dieser phänomenolgisch notwendige Begriff der Rezeptivität steht keineswegs in ausschließendem Gegensatz zur Aktivität des Ich, unter welchem Titel alle spezifisch vom Ichpol ausgehenden Akte zu befassen sind; vielmehr ist die Rezeptivität als unterste Stufe der Aktivität anzusehen. “

${ }^{464}$ Außerdem soll man den Begriff von Aktivität in dem hier einschlägigen, erkenntnistheoretischen Sinne, so Husserl, nicht mit dem Begriff von willkürlicher Handlung im normalen Sinne verwechseln. Vgl. Erfahrung und Urteil, S.89, 235, 300.

${ }^{465}$ Ebd.: „So unterscheiden wir z.B. unter dem Titel Wahrnehmen einerseits das bloße Bewußthaben in originalen Erscheinungen ... In dieser Art ist uns je ein ganzes Wahrnehmungsfeld vor Augen gestellt - schon in purer Passivität. Andererseits steht unter dem Titel Wahrnehmen die aktive Wahrnehmung als aktives Erfassen von Gegenständen, die sich in dem über sie hinausreichenden Wahrnehmungsfelde abheben. “

${ }^{466}$ Es ist zu ergänzen, dass Husserl es nicht vernachlässigt, dass frühere Aktivitäten die Passivität beeinflussen können. Aber um die Analysen zu vereinfachen, hat er diesen Einfluss absichtlich außer Betracht gelassen. Darüber vgl. Erfahrung und Urteil, S.74. 


\section{$\S$ 59. Schlichte Erfassung und Explikation}

\section{a) Die schlichte Erfassung}

Die erste Substufe der Rezeptivität ist die sogenannte schlichte Erfassung. Das ist die Phase, wo das Ich einen sich vom Erfahrungsfeld abhebenden Gegenstand aufmerksam „herausfasst“ 467 , aber seine Details noch nicht „expliziert“. Die Zuwendung zum Gegenstand hat die Tendenz, den Gegenstand nach Details zu betrachten, aber die Aktivierung der Tendenz ist nicht ihre „Verwirklichung “. Es kann sein, dass die Tendenz gehemmt wird: Ich kann in ungünstigen Betrachtungsverhältnissen (z.B. wenn der Gegenstand sich sehr schnell bewegt) den Gegenstand verfolgen und von anderen Mitgegebenen unterscheiden, aber seine eigenen Bestimmungen kaum ,verdeutlichen“. Es kann auch sein, dass das Ich schon mit einer flüchtigen Bekanntschaft mit dem Gegenstand zufrieden ist, da es nur geringes Interesse an ihm hat. ${ }^{468}$ Den Charakter dieser Substufe fasst Husserl wie folgt zusammen:

Die betrachtende Anschauung vor jeder Explikation, die Anschauung, die auf den Gegenstand „,im Ganzen“ gerichtet ist. Diese schlichte Erfassung und Betrachtung ist die unterste Stufe niederer objektivierender Aktivität $[=$ der Rezeptivität - Anm.d.Verf.], die unterste Stufe ungehemmter Auswirkung des Wahrnehmungsinteresses. ${ }^{469}$

Aufgrund der Unterscheidung von Tendenz und ihrer wirklichen Auswirkung hat Husserl die schlichte Erfassung als eine Stufe des kontinuierlich fortgehenden Erkenntnisstrebens extrahiert. Aber soweit die Tendenz, den Gegenstand zu betrachten, nicht durch ungünstige Betrachtungsumstände oder geringes Interesse oder irgendwelche Faktoren gehemmt wird, „kann das Ich bei einer bloß schlichten Betrachtung und Erfassung nicht lange stehen bleiben; vielmehr treibt die Tendenz der Betrachtung alsbald weiter“470; das Ich wird dann in die nächste Substufe der

\footnotetext{
${ }^{467}$ Vgl. [\$36] der vorliegenden Arbeit.

${ }^{468}$ Erfahrung und Urteil, S.113.

${ }^{469}$ Ebd., S.114.

${ }^{470}$ Ebd., S.124.
} 
Rezeptivität eintreten. Das ist die Substufe, die uns besonders interessieren soll, denn in ihr können wir die oben angekündigten syntaktischen Leistungen von Erfahrung ${ }^{471}$ herausarbeiten.

\section{b) Die Explikation}

Bei normal günstigen Erfahrungsverhältnissen wird der Gegenstand nicht einfach „,im Ganzen“, sondern einige seiner Details oder Eigenheiten (Farbe, Gestalt, usw.) treten in den Ich-Blick hervor und affizieren das Ich. Dann lenkt das Ich die Aufmerksamkeit von der Gesamterfassung des Gegenstandes im Ganzen auf Sondererfassungen seiner Eigenheiten. „Es reihen sich Einzelerfassungen aneinander, gerichtet auf Einzelheiten am Gegenstand. Der Gegenstand, und jeder Gegenstand, hat seine Eigenheiten, seine inneren Bestimmungen."(EU, S.124) Z.B. hebe ich im Rahmen des „Themas“ von einer Kugel ihre Rot-Farbe hervor und betrachte sie unter besonderer Erfassung. Wegen der Verteilung der Aufmerksamkeit entstehen auf noetischer und noematischer Seite neue Strukturen.

Der Witz besteht darin, dass das Ich beim Übergang von der Gesamterfassung des Gegenstandes $\mathrm{S}$ zu Sondererfassungen von Eigenheiten $\alpha, \beta$ usw. das $\mathrm{S}$ nicht verlässt, sondern das S noch als das durchgehende Thema beim Erkenntnisstreben gilt. Husserl stellt die Frage:

Was macht aus dem $\mathrm{S}$ das durchgehende Thema in einem ausgezeichneten Sinne, derart daß die $\alpha, \beta$..., wenn sie der Reihe nach erfaßt und so in gewisser Weise auch thematisch geworden sind, gegenüber dem $\mathrm{S}$ jeder Gleichberechtigung entbehren; ja daß sie bloß Themata sind, in denen sich das herrschende Interesse an S konsequent auswirkt, und daß der Übergang zu ihnen nicht ein Sicheinlassen in anderes ist und damit eine Ablenkung und Schwächung des Interesses an S, sondern eine fortgehende Erfüllung und Steigerung desselben? (a.a.O., 126)

Die Antwort auf diese Frage liefern eben die noetisch-noematischen

\footnotetext{
${ }^{471}$ Vgl. [§56] der vorliegenden Arbeit.
} 
Konstitutionsanalysen: Sie beschreiben „die intentionalen Funktionen“" ${ }^{472}$, die es ausmachen, dass sich die Gesamterfassung von $S$ und die Sondererfassungen von $\alpha, \beta$ usw. zu einer Akteinheit, die das $\mathrm{S}$ und die $\alpha, \beta$ usw. in einem Substrat-BestimmungVerhältnis organisiert, zusammenschließen.

Der Schlüsselbegriff für solche Konstitutionsanalysen ist explikative Deckung. Noematisch gesagt ist die explikative Deckung, in Husserl metaphorischer Redeweise, eine „geistige Überschiebung beider Erfaßheiten (S und $\alpha)^{\text {“ }}$ (a.a.O.,128). Überschiebung ist ein geologisches Phänomen, in dem eine Erdschichte älterer Zeiten, die normalerweise tiefer als eine Erdschichte neuerer Zeiten und somit senkrecht von ihr getrennt ist, sich aufwärtsschiebt und mit der neueren Erdschichte ,überschneidet“; durch solche bildliche Metapher der natürlichen Überschiebung will Husserl illustrieren, dass die Noemata von $\mathrm{S}$ und $\alpha$ im Prozess der Explikation nicht zusammenhangslos sind, sondern sich durch ,geistige Überschiebung“ miteinander partiell decken. Auf noetischer Seite ist die Gesamterfassung von S nach dem Übergang $\mathrm{zu}$ den Sondererfassungen von $\alpha, \beta$... noch da und sie wirkt sich noch als thematische Erfassung auf die Sondererfassungen aus. ${ }^{473}$ Husserl zufolge führt die explikative Deckung dazu, dass wir beim kontinuierlichen Übergang von Gesamterfassung des S zu Sondererfassungen von $\alpha, \beta \ldots$ noch auf das $\mathrm{S}$ gerichtet sind und es als Thema des Erkenntnisstrebens im Griff behalten. (a.a.O., 130) Er schreibt:

Nehmen wir einen Gegenstand, S genannt, und die inneren Bestimmungen $\alpha$, $\beta$..., so liefert der von dem Interesse an $\mathrm{S}$ hervorgetriebene Prozeß nicht einfach die Folge: Erfassung S, Erfassung $\alpha$, Erfassung $\beta$, usw., als ob die eine und die andere Erfassung miteinander nichts zu tun hätten, als ob ein Wechsel der Themata erfolgte. Es ist nicht so, wie wenn wir nach dem Erlahmen des Erkenntnisinteresses an einem Gegenstand, nach seiner Überwältigung durch das Interesse an einem zweiten und dann wieder an einem dritten Gegenstand,

\footnotetext{
$472 E U$, S.127: „Es sind also die intentionalen Funktionen $\mathrm{zu}$ beschreiben, die es ausmachen, daß der „Gegenstand“" der Explikation uns in der Sinnesform des „Substrates“ entgegentritt, und in einer ganz anderen die explizierten Momente, nämlich als „Eigenheiten“, „Bestimmungen“ des Gegenstandes, derart daß wir von einer Explikation sprechen können, einer Entfaltung des $\mathrm{S}$ in seine Bestimmungen, davon daß sich das $\mathrm{S}$ bestimme als $\alpha$, als $\beta$ usw. "

${ }^{473}$ Hier gilt das Prinzip des noetisch-noematischen Parallelismus.
} 
uns diesen zuwenden, die sich durch entsprechend kräftige Affektion die Aufmerksamkeit erzwungen haben. (a.a.O., 126)

Und er illustriert das mit einem besonderen Beispiel:

Wir betrachten etwa eine vor uns stehende Kupferschale ... Wenn wir nun die Partialerfassung üben, wie steht es während dessen mit dem Gesamterfassen, dem Erfassen der Schale? Sie bleibt es doch immer, die wir „betrachten“. Immerfort sind wir ihr erfassend zugewendet, aber die Partialerfassungen decken sich mit dieser Gesamterfassung in solcher Art, daß wir in jeder Partialerfassung das Ganze erfassen, sofern es die Eigenheit in der Deckung übergreift und in diesem Übergreifen bewußt ist. (a.a.O., 130)

Husserl zufolge erschöpft aber das Im-Griff-behalten des S nicht all die Charakteristik der Explikation, denn der noematische Inhalt des S bleibt bei dem Im-Griff-behalten nicht unverändert, sondern er nimmt in jedem Schritt der Sondererfassungen etwas Neues in sich hinein. ${ }^{474}$ Das führt zu Husserls Begriff der Sinnesbereicherung.

\section{c) Die Sinnesbereicherung der Explikation und die dabei entstehenden Formen}

\section{von Substrat und Bestimmung}

Das wichtigste Ergebnis der explizierenden Schritte zu $\alpha, \beta$ usw. innherhalb des durchgehenden Themas S ist, dass wir den Gegenstand S immer besser kennenlernen, anders gewendet, dass das Erkenntnisinteresse sich in immer steigendem Maße erfüllt: Zuerst bei schlichter Erfassung fassen wir den Gegenstand einfach als eine Kupferschale 475 auf, bei erster Sondererfassung „erwächst“ er als eine gelbe Kupferschale, bei einer weiteren Sondererfassung von ihrer Gestalt „,erwächst“ sie weiter als runde gelbe Kupferschale usw. „Das S ist nach der Explikation des $\alpha$ das $\mathrm{S} \alpha$, nach Auftreten des $\beta$ das (S $\alpha) \beta$ usw. “ $(E U, 133)$

\footnotetext{
${ }^{474}$ EU, S.132: „Nämlich die Objektauffassung, die im stetigen Im-Griff-behalten des Substrates enthalten ist, nimmt schrittweise alle die herausgehobenen Einzelheiten in sich hinein: das Im-Griff-haben des in der Explikation stehenden Objektes ist nicht ein inhaltlich unveränderliches Im-Griff-haben, also ein Noch-im-Griff-haben desselben, „so wie“ es vor diesem Schritte bewußt war, vielmehr vermöge der ständig neuen Partialdeckungen ein immer wieder verschiedenes. In jedem Schritt wird das einzeln Ergriffene durch die Deckung dem Sinngehalt des Substrates einverleibt.“

${ }^{475}$ Oder mindestens im sehr allgemeinen Sinn als ein zu bestimmder Gegenstand.
} 
Betrachten wir zuerst den einfachsten Fall, wo das Ich schon mit der Sondererfassung von $\alpha$ zufrieden ist und mit der Explikation sogleich aufhört, als es beim S-Thema das $\alpha$ bemerkt hat. Aufgrund dieser aufgehörten Explikation kann das Ich zurückblickend zu dem S zurückkehren, dessen Erfassung nun reicheren Inhalt besitzt als am Anfang (die Kupferschale vs. die rote Kupferschale). Diesen Inhaltszuwachs bezeichnet Husserl als „Sinnesbereicherung“ (a.a.O., 132, 243) des thematischen S; in der Sprache von Ideen I: das Noema (oder der noematische Satz/Sinn) vom S wird um das Noema (oder den noematischen Satz/Sinn) vom $\alpha$ bereichert. Noetisch gesagt wird die Gesamterfassung vom S durch die Sondererfassung vom $\alpha$ ergänzt. $^{476}$

Die dabei stattfindende Sinnesbereicherung hat ihre Ordnung und Form: Das S ist, wie gesagt, der thematische Gegenstand, dessen entsprechender noematischer Satz/Sinn bereichert wird, während das $\alpha$ ein vom S thematisch abhängiger Gegenstand ist, dessen noematischer Satz/Sinn den Satz/Sinn des S bereichert. Aufgrund dieser Ordnung bezeichnet Husserl das erstere als Substrat, während das letztere als Bestimmung/Explikat zu bezeichnen ist. Er schreibt:

Durch den ganzen Prozeß behält das S den Charakter des Themas, und indem wir schrittweise Moment für Moment, Tei für Teil in den Griff bekommen, ist es eben Moment, Teil - allgemein gesprochen - Eigenheit, Bestimmung; so ist es nichts für sich, sondern etwas vom Gegenstande S, etwas aus und in ihm. In der Erfassung der Eigenheiten lernen wir ihn kennen und sie nur als die seinen. Das unbestimmte Thema $\mathrm{S}$ wird in der Entfaltung zum Substrat der hervorgetretenen Eigenheiten, und sie selbst konstituieren sich in ihr als seine Bestimmungen. (a.a.O., 126)

Dadurch wird das $\mathrm{S}$ in der Explikation mit der Substratsform und das a mit der Bestimmungsform überzogen. Husserl zufolge ist die Formbildung von SubstratBestimmung „S- $\alpha$ “, wie wir sehen werden, die Ursprungsstelle der „logischen Kategorien“, die im eigentlichen Sinne nur in der Sphäre des prädikativen Urteilens

\footnotetext{
${ }^{476}$ Ebd., S.133: „Diese Kontinuität [= die schrittweise fortgehende Explikation] ist eine beständige Synthesis der Deckung, die sowohl die Auffassungsinhalte betrifft als die Aktivitäten selbst.“
} 
konstituiert werden. ${ }^{477}$ Die Rede von „Ursprung“ besagt, dass die Formbildung von Substrat-Bestimmung der Urteilsform , $\mathrm{S}$ ist $\alpha^{“ 6}$ epistemisch vorgängig und somit fundierend ist.

Um einen Überblick über den Zusammenhang zwischen der vorprädikativen Explikation und dem prädikativen Urteilen zu gewinnen, gehen wir im folgenden zur Erkenntnisstreben-Stufe des Urteilens. Das nennt Husserl, wie oben schon erwähnt, die Stufe der Spontaneität.

\section{§ 60. Die prädikativen Leistungen des Urteilens}

Husserl zufolge kann das Erkenntnisstreben sein Ziel in der Stufe der Rezeptivität nicht erreichen, denn die Kenntnisnahme, die durch die Explikation erworben wird, ist „,noch nicht zu unserem Besitz geworden, über den wir [intrasubjektiv - Anm. d. Verf.] jederzeit wieder hervorholen und von dem wir Anderen [intersubjektiv - Anm. d. Verf.] Kunde geben können“, ,,auch wenn die Anschauung vorbei ist“. ${ }^{478}$ Das erkenntnisstrebende Ich will also die Kenntnisnahme an dem betreffenden Gegenstand intrasubjektiv und sogar intersubjektiv ,ein für allemal festhalten“. Solche Erkenntnisbesitze erzeugt nur, so Husserl, die Abteilung der prädikativen Spontaneität des Subjekts (bzw. des Ich).

Betrachten wir wieder den einfachsten Fall der Explikation, wo das Ich mit der ersten Sondererfassung vom p zufrieden ist und mit der Explikation sogleich aufhört, als es beim S-Thema das $\mathrm{p}$ bemerkt hat. Wir haben gesehen, dass aufgrund dieser Explikation eine Sinnesbereicherung in Form von Substrat-Bestimmung (S-p) stattfindet. Nun möchte das Ich diese Sinnesbereicherung „dokumentieren“; es setzt dann die Urteilsfähigkeit, nämlich die Ich-Abteilung der Spontaneität in Betrieb und konstituiert ein Urteil der Form „S ist p“, das als Urteilsnoema die Kenntnisnahme der Explikation in inhaltlicher und formaler Hinsicht dokumentiert: Der Substratgegenstand S, der in der Explikation als Explikand fungiert, nimmt nun die Form des prädikativen Subjektes an, während der Bestimmungsgegenstand $\mathrm{p}$, der in der

\footnotetext{
${ }^{477}$ Ebd., S.127.

${ }^{478}$ Ebd., S.232, 233.
} 
Explikation als Explikat fungiert, die Prädikatform übernimmt; außerdem kommt im ,ist“, wie Husserl bemerkt, „die Form der Synthesis [= der explikativen Deckung] ${ }^{479}$ zwischen Explikand und Explikat zum Ausdruck. “ ${ }^{480}$

Das Urteilsnoema unter Betonung seines noematischen Satzes/Sinnes, wie schon erörtert, ist für Husserl eben die Urteilsbedeutung, die der Urteilsakt, wenn er die Funktion der Bedeutungsintention annimmt, einem Ausdruck verleiht. ${ }^{481}$ Daher ist das dokumentierende Urteilsnoema (genauer: der Satz/Sinn des Urteilsnoema) oft schon eine durch Wortlaut ausgedrückte Bedeutung. ${ }^{482}$ In Ideen I spricht Husserl davon, dass solche Bedeutung sich allem „Sinne“ (im Textzusammenhang denkt er hauptsächlich an den noematischen Sinn einer Wahrnehmung) nach Form und Inhalt anpasst und ihn in das Reich des „Logos“ und des „,Begrifflichen“ erhebt. ${ }^{483}$ Dieses in Ideen I nur grob und kurz erwähnte Anpassen und Erheben können wir nun wie folgt reformulieren: Das noematische Gebilde der rezeptiven Erfahrung (Substrat S - Bestimmung p) lässt sich durch das Noema des prädikativen Urteilens ( $S$ ist $\mathrm{p}$ ) in inhaltlicher und formaler Hinsicht dokumentieren.

Ich betone inhaltliche und formale Hinsicht: einerseits bezieht sich das Ich in beiden Akten inhaltlich auf denselben Gegenstand (die Kupferschale, das Rotmoment und den Sachverhalt, dass die Kupferschale rot ist), andererseits spiegelt die prädikative Form ,S ist p“ die explikative Formbildung von Substrat-Bestimmung wider.

Was uns besonders interessiert, ist die formale Hinsicht. Im Prozess des Erkenntnisstrebens findet in der rezeptiven Stufe die Formbildung von SubstratBestimmung statt; unter dem Gesichtspunkt des Erkenntnisstrebens ist diese Formbildung der epistemische Vorgänger bzw. die Ursprungsstelle ${ }^{484}$ der syntaktischen Form „S ist $\mathrm{p}^{“}{ }^{485}$ Wir erinnern uns daran, dass Husserl in Ideen I von syntaktischen

\footnotetext{
${ }^{479}$ Ebd., S. $128-129$

${ }^{480}$ Ebd., S.246.

${ }^{481} \mathrm{Vgl}$. [\$38] und [§43] der vorliegenden Arbeit.

${ }^{482}$ Man möchte Husserl fragen, ob und wieweit das Urteilen an Ausdrücke gebunden sein muss, oder, ob und wieweit das Urteilen als Bedeutungsintention fungieren muss. Leider ist diese Frage in Erfahrung und Urteil absichtlich beiseitegeschoben (EU, S.234). Husserl hat diese Frage, soviel ich weiß, nicht systematisch behandelt.

${ }^{483}$ Hua III/1: Ideen I, S.286.

${ }^{484}$ EU, S.127: „Wir können auch sagen, es ist der Prozeß derjenigen „Evidenz“ aufzuweisen, in dem ursprünglich so etwas erschaut wird wie „Substratgegenstand“ als solcher, und als solcher von so etwas wei „Bestimmungen“. Wir stehen damit an der Ursprungsstelle der ersten der sogenannten ,logischen Kategorien“. “

${ }^{485}$ Ebd., S.247.
} 
Operationen und in Formale und transzendentale Logik von syntaktischen Leistungen der Erfahrung spricht. ${ }^{486}$ Damit sind die Explikation und andere unten noch zu besprechende Operationen/Leistungen in der rezeptiven Stufe gemeint. Genau gesprochen sind aber diese Operationen/Leistungen und die dabei entstandenen noematischen Formen nicht syntaktisch, denn syntaktisch im strengen Sinne sind für Husserl nur die in prädikativer Stufe gebildeten Formen. ${ }^{487}$ Es wäre besser, die im Erkenntnisstreben der Prädikation vorangehenden Formen terminologisch als vorsyntaktische Formen zu bezeichnen.

Zur Vereinfachung haben wir von einem wichtigen Charakter des prädikativen Urteilens abgesehen: Urteilen involviert immer explizit oder implizit begriffliche Fähigkeiten. Das sind Fähigkeiten des Subjekts, Gegenstände unter allgemeine Begriffe zu begreifen. Husserl diskutiert diese Fähigkeiten im Zusammenhang mit einer noch höheren Substufe der prädikativen Spontaneität, d.i. der Stufe des sogenannten „begreifenden Denkens“ “488, in der das Ich allgemeine Gegenstände thematisiert.

\section{§61. Das Allgemeinheitsbewußtsein}

Husserl zufolge involviert jeder Urteilakt, und zwar einer der einfachsten Form „S ist p“, die betreffenden begrifflichen Fähigkeiten:

Wie jeder Gegenstand der Rezeptivität von vornherein dasteht als Gegenstand eines irgendwie bekannten Typus, so findet entsprechend in jeder prädikativen Formung schon eine Bestimmung ,als" das und das statt auf Grund der mit jeder Prädikation untrennbar verflochtenen Ausdrücke und der ihnen zugehörigen allgemeinen Bedeutungen. Wenn wir etwa diesen Wahrnehmungsgegenstand hier als rot bestimmen in einem Wahrnehmungsurteil der einfachsten Form $\mathrm{S}$ ist $\mathrm{p}$, so ist in diesem ,Als-rotBestimmen“ vermöge der Allgemeinheit der Bedeutung „rot“" schon implizite

\footnotetext{
${ }^{486} \mathrm{Vgl} .[\S 46]$ und [§56] der vorliegenden Arbeit.

${ }^{487}$ Erfahrung und Urteil, S.127, 247.

${ }^{488}$ Ebd., S. 381.
} 
enthalten die Beziehung auf das allgemeine Wesen Röte, wenn auch diese

Beziehung noch nicht thematisch zu werden braucht, wie es etwa in der Form geschieht: dies ist ein roter Gegenstand. ${ }^{489}$

Zur Konstitution des Urteils „,die Kugel ist rot“ aufgrund einer Kugel-Wahrnehmung werden also schon die begrifflichen Fähigkeiten des Ich eingesetzt, den wahrgenommenen Substratgegenstand und die wahrgenommene Bestimmung unter die Begriffe von ,Kugel“ und ,,rot“" einzuordnen.

Im Zitat spricht Husserl von ,einem irgendwie bekannten Typus“. Seiner Meinung nach bilden eben diese schon in der Rezeptivität wirksamen Typen die Grundlage für die erst in der Spontaneität eingesetzten begrifflichen Fähigkeiten. ${ }^{490}$ Für Husserl werden die Gegenstände der Erfahrung, ,von vornherein als typisch bekannte erfahren“ 491 : Ich assoziiere den jetzt erfahrenen Gegenstand mit ähnlichen Gegenständen, die mir (oft wegen früherer Erfahrungen) schon bekannt sind; unter der assoziativen Wirkung ist mir der Gegenstand, den ich jetzt erfahre, auch mehr oder weniger bekannt ${ }^{492}$ - ich erwarte, dass er sich so verhalten wird, wie es bei den bekannten Gegenständen typisch der Fall ist. Husserls Beispiel:

Das als individuell Neues Erfahrene ... erinnert an Gleiches (bezw. Ähnliches). Das typisch Erfaßte hat aber auch einen Horizont möglicher Erfahrung mit entsprechenden Bekanntheitsvorzeichnungen, also eine Typik der noch unerfahrenen, aber erwarteten Merkmale: sehen wir einen Hund, so sehen wir sofort sein weiteres Gehaben voraus, seine typische Art zu fressen, zu spielen, zu laufen, zu spring usw. ... obschon wir diesen Hund noch nie gesehen hatten,

\footnotetext{
${ }^{489}$ Erfahrung und Urteil, S.240-241.

${ }^{490}$ Ebd., S.382-383. Dazu vgl. auch Dieter Lohmar, „Genetische Phänomenologie“, in: Husserl-Handbuch, hrsg. von Sebastian Luft und Maren Wehrle, Springer-Verlag GmbH, Stuttgart 2017, S.149-156.

${ }^{491} E U, \mathrm{~S} .385,395$ : „,Daß alle Gegenstände der Erfahrung von vornherein als typisch bekannte erfahren werden, hat seinen Grund in der Sedimentierung aller Apperzeptionen und ihrer habituellen Fortwirkung auf Grund assoziativer Weckung. Assoziation stellt ursprünglich passiv die Synthesis des Gleichen mit dem Gleichen her, und das nicht nur innerhalb eines Feldes der Präsenz, sondern auch durch den ganzen Erlebnisstrom und seine immanente Zeit und alles in ihr je Konstituierte hindurch.“, War vorher in ursprünglicher Vergleichung der Begriff, z.B. Blume, hervortreten, so wird eine neu auftretende Blume auf Grund der assoziativen Weckung des einmal gestifteten Typus „Blume“ als solche wiedererkannt, ohne daß anschauliche Wiedererinnerung an die früheren Fälle der Vergleichung notwendig wäre."

${ }^{492}$ Mit welchen ähnlichen Gegenständen der betreffende Gegenstand assoziiert ist und unter welchen Typus er begriffen wird, hängt in großem Maße von ,hintergründlichen Gewohnheiten“ des Subjekts ab. Ein Urwohner der afrikanischen Wüste könnte, wie in dem Film The Gods must be crazy, eine Cola-Flasche mit Hämmern assoziieren und sie als ein Werkzeug, aber keine Flasche typisieren.
} 
wissen wir im voraus, wie sein Gebiß aussehen wird - nicht individuell bestimmt, sondern eben typisch, sofern wir an ,,dergleichen“ Tieren, ,Hunden“, schon längst und oft erfahren haben, daß sie dergleichen wie ein ,Gebiß“, und ein typisch derartiges haben. ${ }^{493}$

Die Hund-Spezies, oder genauer, die Vor-Spezies des Hundes wird in diesem durch assoziative Ähnlichkeitsdeckung erweckten Bekanntheitscharakter des Gegenstandes (vor-)konstituiert. ${ }^{494}$ Aber um die Spezies ,offiziell“ zu konstituieren und thematisieren, braucht das Ich noch seinen Blick auf den typischen Bekanntheitscharakter zu werfen ${ }^{495}$ und ihn zu „hypostasieren“, d.i. ihn begrifflich zu bearbeiten ${ }^{496}$ und ggf. darüber zu urteilen, wie „Das Tier gehört zu der Spezies Hund“, „Die Spezies Hund kommt diesem Tier zu“" usw. 497

Der Einsatz von begrifflichen Fähigkeiten des Ich heißt bei Husserl noch nicht, dass die betreffenden Allgemeinheiten bzw. Spezies thematisiert werden. Die Urteile (1),,die Kugel ist rot“" und (2),,Rot kommt der Kugel zu“" sind für Husserl wesentlich verschieden, obwohl die begriffliche Fähigkeit, einen Gegenstand unter die Rot-Spezies einzuordnen, in beiden Fällen involviert ist. Denn in (1) bezieht sich „rot“ thematisch auf die Kugel (oder genauer, das Rotmoment der Kugel), während „Rot“ in (2) sich thematisch auf die Allgemeinheit bzw. Spezies Rot bezieht. ${ }^{498}$ Husserl:

Sollen solche Urteile [= Urteile über Allgemeinheiten - Anm. d. Verf.] möglich werden, so setzt das natürlich voraus, daß die Allgemeinheiten, unter die Gegenstände in ihnen begriffen werden, nicht nur passiv vorkonstituiert sind in der Weise ... daß also bloß der Gegenstand mit einem Charakter der Bekanntheit vor uns steht, wobei das Typisch-allgemeine ... als solches

\footnotetext{
${ }^{493}$ Erfahrung und Urteil, S.399.

${ }^{494}$ Ebd., S.382-383, S386. In unserem Beispiel von Hund-Spezies wird nur betrachtet, wie die im Alltag augenfälligtypischen Merkmale von Hunden die Hund-Spezies und somit als Stereotypen (im Sinne von Putnam) die Bedeutung (im Sinne von Putnam) des Terms „Hund“ zum Teil bestimmen. Aber „Hund“ als ein Term von natürlicher Art lässt sich auch wissenschaftlich (in Biologie) definieren; und es kann sein, dass die wissenschaftliche Definition eines Terms von natürlicher Art mit manchen mit ihm verbundenen Stereotypen in Konflikt kommt, z.B. der Term „Wal “. Dementsprechend unterscheidet Husserl (im EU, [\$83b]) zwischen außerwesentlichen Typen (Spezies), die sich durch im Alltag augenfällig-typische Merkmale mehr oder weniger bestimmen lassen, und wesentlichen Typen (Spezies), die eher durch wissenschaftliche Forschung zu charakterisieren sind. Dazu vgl. auch Hilary Putnam, ,,The meaning of 'meaning' “, in: Mind, Language and Reality (Vol.2), Cambridge University Press 1975, S.215ff.

${ }^{495}$ Erfahrung und Urteil, S.391

496 Vorausgesetzt, dass das Ich im seinem Repertoire von Begriffen über den Hund-Begriff verfügt.

${ }^{497}$ Erfahrung und Urteil, S.240-241, 390, 392-393.

${ }^{498}$ Hua XIX/1: Logische Untersuchungen, S.53; Erfahrung und Urteil, S.390.
} 
unthematisch bleibt; vielmehr muß die Allgemeinheit selbst als solche erfaßt sein. 499

Außerdem versucht Husserl in Erfahrung und Urteil zu beschreiben, wie sich die verschiedenartigen Allgemeinheiten (z.B. empirische vs. apriorische Allgemeinheiten, substantivische vs. adjektivische Allgemeinheiten usw. $)^{500}$ durch die Leistungen der Spontaneität konstituieren. Eine ausführliche Diskussion darüber würde von unserem Thema abschweifen, denn wir interessieren uns hier hauptsächlich für die in Stufe der Rezepvitität vollzogenen syntaktischen Leistungen der Erfahrung. Hier genügt es, auf Folgendes hinzuweisen: Husserl zufolge sei die Konstitution der Allgemeinheiten der Schlüssel für das Subjekt, von den niederstufigen Urteilsformen wie „S ist $\mathrm{p}^{\text {“ }}$ in die von ihnen abgewandelten, aber höherstufigen Urteilsformen, nämlich Allgemeinheitsurteilsformen wie ,[Ein S] ist p“, ,,[Alle S] sind p“ vorwärtszugehen. ${ }^{501}$ Auf diese Weise glaubt Husserl, eine Genealogie ${ }^{502}$ bzw. einen Stammbaum der Urteile, durch die Rezeptivität und Spontaneität hindurchgehend, von den niederstufigen bis zu höherstufigen Urteilsformen, zeichnen zu können.

Der Einfachheit halber haben wir bisher die Ursprungsanalysen auf die einfachste Urteilsform „S ist p“ beschränkt. In Erfahrung und Urteil beschäftigt sich Husserl auch mit den Ursprungsstellen von anderen, syntaktisch komplizierteren Urteilsformen. Um einen Überblick über die (vor-)syntaktischen Operationen/Leistungen der Erfahrung zu gewinnen, wollen wir noch einige von ihnen, die für Husserl die ,wichtigsten“ “503 sind, skizzieren.

\section{§ 62. Ursprungsanalysen für kompliziertere Urteilsformen}

\section{a) Ursprungsanalysen für die Urteilsform ,,S ist p und q und $r$...6}

In der Explikation wird das Erkenntnisinteresse normalerweise nicht schon

\footnotetext{
${ }^{499}$ Erfharung und Urteil, S.382-383.

${ }^{500}$ Ebd., III. Abschnitt, Kap.I und II.

${ }^{501}$ Ebd., S.382, 390.

${ }^{502}$ Ebd., S.1, 9, 37, 50-51.

${ }^{503}$ Der Titel des Kapitels (I. Kaptel des II. Abschnitts), wo Husserl diese (vor-)syntaktischen Leistungen betrachtet, heißt „Die allgemeinen Strukturen der Prädikation und die Genesis der wichtigsten kategorialen Formen“.
} 
dadurch befriedigt, dass das Ich nur eine einzige Bestimmung p des Gegenstands $\mathrm{S}$ feststellt, sondern es wird von p aus weiter zu q, r, s usw. fortlaufen. In diesem Prozess sind q, r, s nicht für sich, sondern als zum Substratgegenstand S gehörige Betimmungen erfasst. Mit Husserls eigenen Worten:

Das Substrat S [bleibt] im Griff, und im Ergreifen der Explikate bereichert es sich fortlaufend um $\mathrm{p}, \mathrm{q}, \mathrm{r}$, wenn jedes einzelne nicht nur für sich ergriffen ... wobei sie auch untereinander als dem $\mathrm{S}$ zugehörige passiv in eine synthetische Überschiebung treten. ${ }^{504}$

Das heißt, die Bestimmungen stehen mit dem Substrat $\mathrm{S}$ jeweils in einer explikativen Deckung, wobei die Formbildung von Substrat-Bestimmungen, die ich durch die Formel „,S - p-q-r ... “ repräsentieren möchte, stattfindet. Um die dabei stattfindende Sinnesbereicherung von S prädikativ zu dokumentieren, erzeugt das Ich ein Urteil der Form , $\mathrm{S}$ ist $\mathrm{p}$ und $\mathrm{q}$ und $\mathrm{r}$ “ oder,, $\mathrm{S}$ ist $\mathrm{p}$ und dasselbe $\mathrm{S}$ ist $\mathrm{q}$ und dasselbe $\mathrm{S}$ ist $\mathrm{r}$ “. 505

Stillschweigend haben wir angenommen, dass die Aufmerksamkeit des Ich auf die Bestimmungen p, q, r, s usw. gleichmäßig verteilt wird - dabei sind die Bestimmungen für das Erkenntnisinteresse gleich wichtig. Doch es ist auch möglich, dass in diesem Explikationsprozess nur einige Bestimmungen wichtig sind, während die anderen das Ich weniger oder gar nicht interessieren. Husserl zufolge kann diese ungleichmäßige Verteilung des Erkenntnisinteresses den Ursprung der Attribution erklären.

\section{b) Ursprungsanalysen für Attribution enthaltende Urteilsformen}

Das Ich kann auch im explikativen Prozess vom S die Bestimmung p nur nebenbei berühren und das Hauptinteresse auf die Bestimmung q legen. Mit Husserls eigenen Worten, das q ,ist vorzugsweise in den Blick genommen“. Freilich wird hier, wie in anderen Fällen der Explikation, der Substratgegenstand S als Hauptthema im Griff beibehalten; und die Bestimmungen $\mathrm{p}$ und $\mathrm{q}$ stehen auch jeweils in explikativer Deckung mit dem S, aber mit verschiedenen Auffälligkeitscharakteren. Die dabei

\footnotetext{
${ }^{504}$ Erfahrung und Urteil, S.256.

${ }^{505}$ Es ist zu bemerken, dass Husserl zwischen den beiden Urteilsformen hinsichtlich der Erfassungsschritte eine substile Differenz macht, die in [§51a] und [§51c] von Erfahrung und Urteil beschrieben wird.
} 
stattfindende (vor-)syntaktische Formbildung möchte ich durch die Formel ,,(Sq)_p“ repräsentieren. Der Unterstrich „_ “ "deutet darauf hin, dass die Bestimmung p das Ich weniger interessiert.

Das entsprechende Ergebnis auf der prädikativen Stufe ist also nicht ein einfaches ,S ist p und q“, sondern „,der aktive Vollzug der Synthesis $S$ ist $q$ wird den Charakter des Hauptsatzes annehmen und der der Synthesis $S$ ist $p$ den des Nebensatzes“. ${ }^{506}$ Ein solches Urteil wird durch die Satzform „,S, welches $p$ ist, ist q“ ausgedrückt.

Es kann auch sein, dass das S schon in einer früheren Explikation als p bestimmt und diese Kenntnisnahme als Besitz beibehalten wird. In einer neuen Explikation steht das $\mathrm{S}$ von vornherein als ein als p Bekanntes in explikativer Deckung mit q. Aufgrund dieser Deckungweise vollzieht das Ich das Urteil der Form „Sp ist q“ (z.B. die rote Kugel ist groß).

Hier hat Husserl eine subtile Differenz zwischen ,,S, welches $p$ ist, ist q“ und ,,Sp ist q" gemacht. Beide enthalten attributive Komponente, in der Form von Relativsatz oder Adjektiv. Nach Husserl wird im ersteren Fall ein Übergang von S zu p noch in gewissem Maße spontan vollzogen, obwohl nur im Modus von „Nebenbei“; im letzteren Fall wird das S aufgrund vorheriger Prädikation direkt als p erfasst, „das S wird sogleich als p genommen und spontan nur der Übergang zum q vollzogen. “507

Außerdem hat Husserl auch auf die Möglichkeit von Attribution hingewiesen, die auf der Bestimmungsseite haftet. „Wir urteilen $\mathrm{S}$ ist p; es werde nun ein thematisch bestimmmendes Interesse für p erweckt, und dieses bestimmt sich als $a$, während doch das Interesse für das S festgehalten und übergeordnet bleibt. “ ${ }^{508}$ Hier geht das Erkenntnisinteresse in die Bestimmung $\mathrm{p}$ hinein, d.h. es substantiviert p. Husserl betont, dass im Rahmen der ganzen Explikation das substantivierte p nur als untergeordnetes Substrat angesehen werden kann, da ihm das S als Hauptthema der Explikation

\footnotetext{
${ }^{506} E U$, S.271: „In der prädikativen Sphäre wird dann dementsprechend nicht ein einfach fortlaufendes Bestimmen der Form „,S ist $\mathrm{p}$ und q" statthaben, sondern der aktive Vollzug der Synthesis S ist q wird den Charakter des Hauptsatzes annehmen und der der Synhesis S ist $\mathrm{p}$ den des Nebensatzes ... Ausdrücke, die auch hier wieder, wie klar ersichtlich, primär nichts Sprachliches bezeichnen, sondern die dem sprachlichen Ausdruck Bedeutung gebende Weise kategorialer Synthesis, die in der sprachlichen Hypotaxe ihren Ausdruck finden kann, aber nicht muß ... “ ${ }^{507}$ Ebd., S.274.

${ }^{508}$ Ebd., S.275.
} 
übergeordnet bleibt. Die dabei stattfindende (vor-)syntaktische Formbildung kann man durch die Formel „S-(p- $a)^{\text {“ }}$ repräsentieren. Auf der prädikativen Stufe wird das

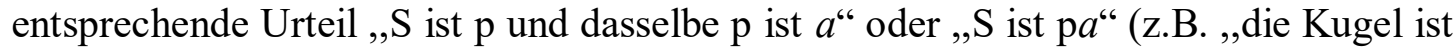
dunkel-rot") erzeugt. In der Sprache wird solche Attribution zweiter Stufe oft durch Adverbien gekennzeichnet.

\section{c) Der Unterschied zwischen „Ist“"-Urteil und „Hat"-Urteil}

Bisher haben wir bei der Diskussion über explizierende Betrachtung die Form des „Ist“-Urteils wie „S ist p“ bevorzugt. Husserl bemerkt, dass es sich in der Sprache bei „Ist“-Urteilen im hier relevanten Sinne um unselbständige Bestimmungen (bzw. Momente) des Substratgegenstandes handelt; wenn man einen selbständigen Teil $\mathrm{t}$ dem S zusprechen will, verwendet man normalerweise die Form des „Hat“-Urteils wie „S hat $t^{\text {" }}$ (,das Haus hat ein Fenster"). Husserl zufolge ist das „Hat"Urteil ,gleichursprünglich“509 mit dem „Ist“-Urteil. Alles, was nach dem in [§62a] und [§62b] Ausgeführten für das bevorzugte „Ist“"-Urteil gilt, gilt mutatis mutandis auch für das „Hat“-Urteil. Z.B. wird ein ursprünglichstes Urteil der Form,,S hat $t_{1}$ und $t_{2}$ und $t_{3} \ldots$.. auch dirket aufgrund einer Explikation, die die (selbständigen) Teile $t_{1}, t_{2}, t_{3} u s w$. in Sonderfassungen betrachtet, vollzogen.

Vielleicht hätte Husserl auch die Urteilsformen von „SV(O)“ (z.B. ,Der Hund schwimmt“ und „Die Katze hetzt die Ratte“), bei denen es sich um eine ,,verbale“ Bestimmung handelt, erwähnen und diskutieren sollen, ob diese Urteilsformen mit dem „Ist“- und „Hat“-Urteil gleichursprünglich sind; oder kann man die Urteilsform „SV“ (,der Hund schwimmt“) auf ein „Ist“-Urteil als kanonische Form (,der Hund ist schwimmend“) reduzieren und die Form „SVO“, die eigentlich eine beziehende Urteilsform und im folgenden Paragraph zu besprechen ist, auf eine kanonische Form wie „S ist (V-end O)“ („die Katze ist [die Ratte hetzend]“) zurückführen? Es ist unklar, wie Husserl diese Frage beantworten würde; denn in

\footnotetext{
${ }^{509}$ Ebd., S.262: „Im Unterschied vom Ist-Urteil tritt aber im Hat-Urteil nicht nur ein einziger selbständiger Gegenstand in der Kernform der Substantivität auf, nämlich das Subjekt, sondern noch ein zweiter auf der Prädikatseite als Objekt. Genetisch gesehen ist das „Hat“-Urteil, sofern es sich auf selbständige Teile eines Substrates bezeiht, gleichursprünglich mit dem „Ist“"Urteil.“"
} 
Erfahrung und Urteil wird die Frage nicht behandelt.

\section{d) Ursprungsanalysen für beziehende Urteilsformen}

Die explizierende Betrachtung, die die inneren Bestimmungen eines Gegenstandes enthüllt, ist eine Exploration in den Innenhorizont ${ }^{510}$ des Gegenstandes. Das Ich kann aber auch in den Außenhorizont des Gegenstandes hineingehen: es macht die „umgebenden“ Gegenstände mit thematisch und betrachtet den thematischen Gegenstand in Beziehung zu ihnen. ${ }^{511}$ Dabei handelt es sich um die Betrachtung von relativen Bestimmungen. Husserl:

Es erwachsen dabei im Gegensatz zu seinen inneren Bestimmungen, Explikaten, die relativen Bestimmungen, die auslegen, was er in bezug auf andere Gegenstände ist: der Bleistift liegt neben dem Tintenfaß, er ist länger als der Federhalter usw. ${ }^{512}$

Die rezeptive Stufe, in der das Ich den Außenhorizont des Gegenstandes exploriert, ist bei Husserl als beziehende Betrachtung (im Vergleich zu explizierender Betrachtung) bezeichnet. In dem Fall, wo die beiden in einer Beziehung stehenden Gegenstände im Wahrnehmungsfeld gegeben sind, ist schon eine noematische Einheit von beiden auf der Stufe der Passivität konstituiert. ${ }^{513}$ Husserl zufolge bildet diese passiv-konstituierte Einheit die Grundlage für die erst in der Rezeptivität stattfindende, beziehende Betrachtung und dann letztlich für den erst in prädikativer Stufe (vor-)kostituierten Sachverhalt, daher wird sie als fundamentum relationis ${ }^{514}$ bezeichnet.

Bei beziehender Betrachtung wirft das Ich den Blick auf ein Glied der Einheit und dann auf ein anderes, während das Ersterfasste noch im Griff behalten wird. Dabei ,hat eine synthetische Überschiebung der beiden Erfasstheiten, des im Griff behaltenen

\footnotetext{
${ }^{510}$ Vgl. [\$41] der vorliegenden Arbeit. Mehr über den Horizontsbegriff vgl. David Woodruff Smith and Ronald McIntyre, Husserl and Intentionality, S.227 ff.

${ }^{511}$ Erfahrung und Urteil, S.115.

512 Ebd., S.115-116.

${ }^{513}$ Ebd., S.178-180. Streng genommen gilt das auch für die explizierende Betrachtung: Bevor die Explikation statthat, ist schon eine Einheit des Gegenstandes und der Bestimmung in der Passivität konstituiert. In gewissem Sinne ist die Explikation eine in der Rezeptivität stattfindende Bearbeitung einer in Passivität erzeugten Einheit. Nach Süßbauer ist die Einheit, die in der Passivität vorgegeben und in der Rezeptivität bearbeitet wird, eben das, was Husserl in Erfahrung und Urteil als Sachlage bezeichnet. Vgl. Alfons Süßbauer, Intentionalität, Sachverhalt, Noema, S.259, 161-262, 277-279 und [\$66] der vorliegenden Arbeit.

${ }^{514}$ Erfahrung und Urteil, S.178, 288. Vgl. Alfons Süßbauer, Intentionalität, Sachverhalt, Noema, S.283ff.
} 
Hauptthemas (Federhalter) und des bezüglichen Themas (Tisch bezw. Bleistift) statt“" ${ }^{515}$ Diese Überschiebung ist eine bestimmte Deckung zwischen dem Hauptthema und dem bezüglichen Thema z.B. hinsichtlich des räumlichen Verhältnisses (A liegt auf B). Dann findet die Formbildung von ,Substrat A - liegt auf - Substrat B“ statt; das Element „liegt auf“ entspricht der dabei konstituierten relativen Bestimmung. Um die relativen Bestimmungen von den inneren Bestimmungen und somit die beziehende von der explikativen Betrachtung zu unterscheiden, wählt Husserl wieder eine metaphorische Redeweise:

An dem Gegenstand, so sagten wir, erfassen wir die relativen Beschaffenheiten, ebenso wie wir die inneren Explikate an ihm erfassen. Aber die inneren Bestimmungen erfassen wir zugleich als in ihm enthalten, mit ihm in partialer Deckung; die relativen dagegen sind nie in dem Gegenstand, sondern sie erwachsen erst beim Übergang zum relativen Objekt, strecken ihm sozusagen „Fangarme“ entgegen. ${ }^{516}$

Auf der prädikativen Stufe wird das entsprechende Urteil „A liegt auf B“( (z.B. ,,der Federhalter liegt auf dem Tisch“) erzeugt und dabei vor-konstituiert ${ }^{517}$ sich ein Relationssachverhalt als das Endergebnis des „Vorgangs“ von Passivität über Rezeptivität bis zur prädikativen Spontaneität. Der Urteilsakt, der den Sachverhalt (vor-)konstituiert, hat also konstitutionsanalytisch sein Fundament in der Passivität und Rezeptivität. ${ }^{518}$

\section{e) Ursprungsanalysen für Identitätsurteil}

Im Zug der explizierenenden bzw. beziehenden Betrachtung erwachsen Formen von inneren oder relativen Bestimmungen und dann Urteilsformen, die den Substratgegenstand innerlich oder relativ bestimmen. Von diesen bestimmenden

\footnotetext{
515 Ebd., S.176.

${ }^{516}$ Ebd., S.178-179.

517 Streng genommen wird der Sachverhalt konstituiert (ohne ,vor“) erst durch eine ausschließlich in der Spontaneität stattfindende Substantivierung. Nachdem ich z.B. urteilsmäßig festgestellt habe, dass das Wetter gut ist, kann ich weitere Urteile fällen, die alle sich um diesen Wetter-Sachverhalt drehen, wie ,es freut mich, dass das Wetter gut ist“, „,das, dass das Wetter gut ist, ist der Grund dafür, dass wir Fahrrad fahren“" usw. Dabei wird der Wetter-Sachverhalt substantivert und in weiteren Zusammenhängen bestimmt. Darüber vgl. EU, S.283-284.

${ }^{518}$ Erfahrung und Urteil, S.177-178, 288.
} 
Urteilsformen unterscheidet Husserl die Identitätsurteil der Form „A ist identisch mit $\mathrm{B}^{\prime}$.

Im Verlauf der explikativen oder beziehenden Betrachtung eines thematischen Gegenstandes sind wir in der Regel auf ihn gerichtet als etwas Identisches immer neuer Bestimmungen, „ohne dass wir dabei auf seine sich dabei erhaltende Identität selbst gerichtet wären“. Wird etwa das S als p und weiterhin als q usw. bestimmt, so deckt sich automatisch das als $\mathrm{p}$ bestimmte $\mathrm{S}$ mit dem als q bestimmten ,in einer gewissen Selbstverständlichkeit““.519

Aber manchmal kann sich das Bedürfnis einstellen, die Identität des einmal als $\mathrm{p}$ bestimmten $\mathrm{S}$ mit dem das andere Mal als q bestimmten ausdrücklich zu bestätigen, z.B. wenn die beiden Explikationen, in denen das S jeweils als p und q bestimmt wird, eine ganze Weile getrennt sind und das Ich die Frage stellt, „Ist der Gegenstand, welcher $\mathrm{p}$ ist, identisch mit dem, welcher q ist?“ Das Ich muss über die Identitätsdeckung „S-p $=\mathrm{S}-\mathrm{q}^{\text {“ }}$ reflektieren und sie überprüfen; wenn das Ich durch weitere Erfahrungen feststellt, dass die Identitätsdeckung problemlos ist, kann es auf der prädikativen Stufe das Urteil „das Sp ist identisch mit dem Sq“ (z.B. „die rote Kugel ist identisch mit der großen) erzeugen. In diesem Sinne enthalten Identitätsurteile der Form ,a=b“ (im Vergleich $\mathrm{zu}$ der Form ,,a=a“c), mit Frege $\mathrm{zu}$ sprechen, ,,wertvolle Erweiterungen unserer Erkenntnis“"520; denn diese Urteile gelten nicht a priori und sind nach Kant analytisch zu nennen (wie ,a $\mathrm{a}=\mathrm{a}^{“}$ ), sondern sie sind nur durch Erfahrungen zu bestätigen und dann als empirische Erkenntnisse zu bezeichnen.

\section{§ 63. Zusammenfassung des Kapitels}

In diesem Kapitel haben wir, durch die Stufen des Erkenntnisstrebens hindurchgehend, verschiedene Urteilsformen genetisch auf ihre vorprädikativen Ursprünge zurückgeführt. Was uns besonders interessiert, ist, dass eine Serie von prädikativen Operationen/Leistungen entsprechende noetische Vorgänger in der

\footnotetext{
${ }^{519}$ Ebd., S.280.

${ }^{520}$ Gottlob Frege, ,Über Sinn und Bedeutung“, in: Gottlob Frege. Funktion, Begriff, Bedeutung, hrsg. von Günther Patzig, Vandenhoeck \& Ruprecht, Göttingen 2008.
} 
rezeptiven Stufe der Erfahrung haben. Auf noematischer Seite haben eine Familie von Urteilsformen, wie wir im [§62] herausgestellt haben, parallele vor-syntaktische Gebilde in der rezeptiven Stufe der Erfahrung.

Dieser Parallelismus zwischen den syntaktischen Leistungen des Urteilens und den vor-syntaktischen Leistungen der Erfahrung liefert uns den Schlüssel, im folgenden Kapitel die Rede des Grammatikalitätsphänomens von Bedeutungsintentionen (Urteilsakten) auf bedeutungerfüllende Akte zu übertragen. 


\section{KAPITEL}

\section{GRAMMATIKALITÄTSTHESEN IM BEREICH DER \\ BEDEUTUNGERFÜLLENDEN AKTE}

\section{§64. Vor-syntaktische Formen vs. vor-grammatische Formen}

Wir haben erkannt, dass einer Reihe von syntaktischen Urteilsformen parallele vor-syntaktische Formen entsprechen, die Husserl als vorprädikative Ursprungsstelle der betreffenden Urteilsformen erklärt. Ziehen wir die Unterscheidung zwischen syntaktischen Formen (=Funktionsformen) und Kernformen (= Bedeutungskategorien) wieder in Betracht ${ }^{521}$, dann können wir bemerken, dass auch die Kernformen ihre Gegenstücke in der Rezeptivität finden können.

Die Kernform einer Bedeutung entscheidet, welche syntaktischen Formen die betreffende Bedeutung in einer Urteilseinheit übernehmen können. ${ }^{522}$ Z.B. kann die adjektivische Bedeutung (Kernform) ,rot“ in einer Urteilseinheit entweder als Prädikation oder als Attribution (syntaktische Form) fungieren, aber nicht ohne weiteres die Subjekt- oder Objekt-Funktion übernehmen, ${ }^{523}$ sonst würde nur eine sinnlose Bedeutung, die eigentlich gar keine einheitliche Bedeutung ist, generiert werden.

Bei der entsprechenden Formbildung, die in der Rezeptivität stattfindet, bemerkt man ein paralleles Phänomen. Z.B. kann das Rotmoment p eines Gegenstandes S, das bei der Explikation als Bestimmung des $\mathrm{S}$ erfasst wird, für das Erkenntnisstreben entweder im Hauptgewicht (quasi-prädikativ - vgl. [\$59c] der vorliegenden Arbeit) oder nur im Nebenbei (quasi-attributiv - vgl. [§62b]) den noematischen Satz/Sinn des $\mathrm{S}$ bereichern, aber nicht ohne weiteres die Stellung des haupt- oder neben-thematischen Substrats einnehmen, ${ }^{524}$ sonst würde gar keine Sinnesbereicherung in der rezeptiven Stufe erworben werden. Ähnliches gilt auch für das bei der Explikation zunächst als Substrat Erfasste: es kann entweder als ein (der Subjektsform entsprechendes)

\footnotetext{
${ }^{521} \mathrm{Vgl}$. [§13] der vorliegenden Arbeit.

522 Erfahrung und Urteil, S.248.

${ }^{523}$ Dafür muss sie zuerst nominalisiert werden. Vgl. [§13] der vorliegenden Arbeit

${ }^{524}$ Dafür muss die Bestimmung zuerst substantiviert werden. Vgl. Erfahrung und Urteil, S.275, 284.
} 
hauptthematisches Substrat oder ein (der Objektsform entsprechendes) nebenthematisches Substrat, aber nicht ohne weiteres als eine (der prädikativen oder attributiven Form entsprechende) Bestimmung fungieren. Das entsprechende Phänomen auf der prädikativen Stufe ist, dass eine substantivische Bedeutung entweder als Subjekt oder Objekt in einer Urteilseinheit, aber nicht ohne weiteres als Prädikation oder Attribution fungieren kann, sonst würde gar keine einheitliche Urteilsbedeutung erzeugt werden.

Die Urteilsformen, deren Ursprungstelle im obigen Kapitel enthüllt sind, haben also in der Rezeptivität nicht nur Formen, die den in ihnen enthaltenen syntaktischen Formen entsprechen und als vor-syntaktische Formen bezeichnet werden, sondern auch Formen, die den in ihnen enthaltenen Kernformen entsprechen und somit als VorKernformen oder Vor-Bedeutungskategorien oder vor-grammatsiche Formen bezeichnet werden dürfen.

Die Herausarbeitung dieser vor-grammatischen Formen ist zugleich Rechtfertigung für die folgenden Grammatikalitätsthesen.

\section{§65. Das (Vor-)Grammatikalitätsphänomen der noematischen Sätze/Sinne von bedeutungerfüllenden Akten}

Bisher hatten wir bei unseren Analysen über vor-prädikative Erfahrung hauptsächlich die äußere Wahrnehmung im Auge, denn Wahrnehmung hat eine besondere Stellung im Erkenntnisstreben. Doch das oben Ausgeführte (besonders die Enthüllung der vor-grammatischen Formen der Erfahrung) gilt mutatis mutandis für andere Anschauungsarten wie innere Wahrnehmung, Erinnerung, Phantasie usw. ${ }^{525}$ Deshalb brauchen wir uns bei der Rede von vor-grammatischen Formen der Erfahrung nicht auf äußere Wahrnehmung zu beschränken. Da die Anschauungsakte die Klasse der möglichen bedeutungerfüllenden Akte bilden ${ }^{526}$, dürfen wir auch allgemein von vor-grammatischen Formen der bedeutungerfüllenden Akte sprechen.

\footnotetext{
${ }^{525}$ In einem von Husserl erkannten, sehr weiten Sinne vom Erfahrungsbegriff ist der Term „Erfahrung“ mit dem Term ,Anschauung“ sogar umfangsgleich. Vgl. Erfahrung und Urteil, S.21-22.

${ }^{526}$ Vgl. [\$5] der vorliegenden Arbeit.
} 
Die vor-grammatischen Formen, die schon in der Erfahrungsstufe des Erkenntnisstrebens (besonders in der explizierenden bzw. beziehenden Betrachtungsstufe) „erwachsen“, sind zugleich Formen der Stoffe ${ }^{527}$ der dabei bereicherten noematischen Sätze/Sinne ${ }^{528}$. Bei der explizierenden Betrachtung wird z.B. der noematische Satz/Sinn vom S mit der vor-grammatischen substantivischen Form und der noematische Satz/Sinn vom p mit der vor-grammatischen adjektivischen Form umgekleidet; und die Gesamtform des dabei bereicherten noematischen Satzes/Sinnes lässt sich durch $\left[[\mathrm{S}]_{\mathrm{n}^{*-}}[\mathrm{p}]_{\mathrm{adj}^{*}}\right]_{\mathrm{s}} / \mathrm{n}^{*}$ charakterisieren. Das Sternzeichen deutet an, dass die hier gekennezeichneten Formen nicht grammatische Formen im eigentlichen Sinne, sondern vor-grammatische sind. Das Suffix , ,s*/n*“ weist darauf hin, dass die Form des zusammengesetzten Satzes/Sinnes etwas ,elastisch“ ist: er trägt die vor-grammatische Form, die sich in der prädikativen Stufe durch die Form $\left[[\mathrm{S}]_{\mathrm{n}} \text { ist }[\mathrm{p}]_{\mathrm{adj}}\right]_{\mathrm{s}}($,,der Baum ist grün“) oder die Form $\left[[S]_{n}[\mathrm{p}]_{\mathrm{adj}}\right]_{\mathrm{n}}($,,der Baum, der grün ist“) festhalten lässt; das Ich macht die Entscheidung, welche der beiden prädikativ grammatischen Formen dafür auszuwählen ist. ${ }^{529}$ Trotz dieser elastischen Entscheidungsfreiheit ist aber die Komplikation der enthaltenen noematischen Sätze/Sinne dabei beschränkt: der substantivische noematische Satz/Sinn $[\mathrm{S}]_{\mathrm{n}^{*}}$ wird in der explizierenden Betrachtung mit dem adjektivischen noematischen Satz/Sinn $[\mathrm{p}]_{\text {adj* }}$ nur zu einem umfassenden noematischen Satz/Sinn zusammengesetzt werden, der prädikativ entweder durch einen propositionalen oder substantivischen Urteilssatz/Urteilssinn 530 festzuhalten ist; variiert man die Form nach Belieben, dann bekommt man nur eine Sukzession, aber keine Einheit von noematischen Sätzen/Sinnen der Erfahrung (z.B. zwei thematische Substrate: zuerst $[\mathrm{S}]_{\mathrm{n}^{*}}$, dann $\left.[\mathrm{p}]_{\mathrm{n}^{*}}\right)$. Gleiches gilt auch für die anderen vor-syntaktischen und somit vor-grammatischen Formen, die wir im letzten Kapitel (besonders im [§62]) betrachteten.

Nach dem oben Ausgeführten können wir feststellen:

(1) Einerseits unterstehen die noematischen Sätze/Sinne von

\footnotetext{
${ }^{527} \mathrm{Vgl}$. [§13] der vorliegenden Arbeit.

${ }^{528}$ Vgl. [\$59c] der vorliegenden Arbeit.

${ }^{529} \mathrm{Vgl}$. [§62b] der vorliegenden Arbeit.

${ }^{530}$ Urteil in dem im [§20] erwähnten erweiterten Sinne, wobei substantivische Bedeutung auch als Urteil zählt.
} 
bedeutungerfüllenden Akten verschiedenen vor-grammatischen Formen (=VorBedeutungskategorien), die wir, in Anlehnung an [§47], als vorgrammatischnoematische Kategorien bezeichnen können.

(2) Andererseits schreiben diese vorgrammatisch-noematischen Kategorien Komplikationsgesetze vor, die bestimmen, welche noematische Sätze/Sinne (von bedeutungerfüllenden Akten) mit welchen noematischen Sätzen/Sinnen (von bedeutungerfüllenden Akten) sich zu einem einheitlichen Satz/Sinn von welcher vorgrammatisch-noematischen Kategorie zusammenschließen können.

Wir können (die Grammatikalitätsthese der noematischen Sätze/Sinne von bedeutungerfüllenden Akten) wie folgt einführen:

(Die Grammatikalitätsthese der noematischen Sätze/Sinne von bedeutungerfüllenden Akten)

Die noematischen Sätze/Sinne von bedeutungerfüllenden Akten sind gewissen durch vorgrammatisch-noematische Kategorien bestimmten vorgrammatischen Gesetzen unterworfen. Das bezeichnen wir als das VorGrammatikalitätsphänomen (bezüglich) der noematischen Sätze/Sinne von bedeutungerfüllenden Akten.

In VI. LU erwähnt Husserl „das intuitive Gegenstück der rein logisch grammatischen Gesetzmäßigkeit"“531, wobei er auch schreibt:

Der reinen Formenlehre der Bedeutungen entspricht eine Formenlehre der Anschauungen, in welcher die primitiven Typen von einfachen und komplexen Anschauungen durch intuitive Generalisation als möglich aufgezeigt und die Gesetzmäßigkeiten ihrer sukzessiven Komplikation zu immer neuen und komplizierteren Anschauungen bestimmt werden müßten. ${ }^{532}$

Wir können dieses ,intuitive Gegenstück der rein logisch grammatischen Gesetzmäßigkeit“" durch unsere Rede des Vor-Grammatikalitätsphänomens der noematischen Sätze/Sinne von bedeutungerfüllenden Akten erklären; die im Zitat konzipierte Formenlehre der Anschauungen soll eben dieses Vor-

\footnotetext{
${ }^{531}$ Hua XIX/2: Logische Untersuchungen, S.710.

${ }^{532}$ Ebd., S.711.
} 
Grammatikalitätsphänomen untersuchen.

\section{§ 66. Das (Vor-)Grammatikalitätsphänomen des bestimmbaren $X$ von bedeutungerfüllenden Akten}

Ein anderes wichtiges noematiches Moment von bedeutungerfüllenden Akten ist das bestimmbare X. Ich nehme an, ${ }^{533}$ dass die in [\$42] aufgestellte (Spezifikation des bestimmbaren $X$ der Wahrnehmung) sich auf alle bedeutungerfüllenden Akte mutatis mutandis übertragen lässt:

(Spezifikation des bestimmbaren X des bedeutungerfüllenden Aktes) $)^{534}$

In jedem bedeutungerfüllenden Akt wird ein X konstituiert, das entweder:

(i) mit dem wirklichen Gegenstand, der so ist, wie er in dem

bedeutungerfüllenden Akt intendiert ist, zusammenfällt, oder

(ii) es verhielte sich so, wie in (i) beschrieben, wenn der bedeutungerfüllende

Akt veridisch wäre.

Ein bedeutungerfüllender Akt könnte in seinem Verlauf von niederen zu höheren Stufen verschiedene, aber miteinander zusammenhängende Gegenstände als das bestimmbare X konstituieren. Z.B. wird bei der schlichten Erfassung einer veridischen Wahrnehmung ein Baum als individueller Erfahrungsgegenstand konstituiert, in der explizierenden Betrachtung wird aber noch seine Farbe konstituiert - aber beide, der Baum und seine Farbe, sind nicht zusammenhangslos, sondern sie stehen im SubstratBestimmung-Verhältnis und somit bilden eine gegenständliche Einheit, die Husserl auch als Sachlage ${ }^{535}$ bezeichnet. Diese Sachlage ist für Husserl das, „was einem solchen Sachverhalt [z.B. der Baum ist grün - Anm. d. Verf.] in der Rezeptivität entspricht“،. Die Sachlage ist also der Vorgänger des erst in prädikativen Stufe konstituierten Sachverhalts, der das bestimmbare $\mathrm{X}$ des Urteilens ist ${ }^{536}$; auf der prädikativen Stufe kann das Subjekt dieselbe Sachlage auf verschiedene Weisen bearbeiten: es kann urteilen, ,,der Baum ist grün“ oder ,,die grüne Farbe kommt dem

\footnotetext{
${ }^{533}$ Die Annahme lässt sich wie folgt begründen: Auch andere Anschauungsakte, die nicht Wahrnehmungen sind, enthalten das noematische X; im Rahmen der transzendentalen Reduktion wollen wir eine existenzneutrale Spezifikation des X auch für diese anderen Anschauungsakte angeben; diese Anforderung erfüllt die folgende Spezifikation des bestimmbaren $X$ des bedeutungerfüllenden Aktes.

${ }^{534}$ Kann man auch bei nicht-setzenden Akten wie Phantasie von Veridizität sprechen? Wenn man diese Spezifikation auch auf nicht-setzende Akte anwenden will, dann braucht man die Rede von Veridizität zu erweitern, man könnte z.B. festsetzen, dass ein Phantasieakt veridisch ist, wenn sein setzendes Gegenstück veridisch ist. Oder man beschränkt diese Spezifikation einfach auf setzende Akte.

${ }^{535}$ Erfahrung und Urteil, S.285.

${ }^{536}$ Vgl. [§43a] der vorliegenden Arbeit.
} 
Baum zu“. Man kann nicht ausschließen, dass die Sachlage auch als das bestimmbare $\mathrm{X}$ der Wahrnehmung (insbesondere in der höheren rezeptiven Stufe) angesehen werden kann. ${ }^{537}$ Wir haben hier zwei Kandidaten für das bestimmbare $\mathrm{X}$, den Substratgegenstand und die den Substratgegenstand ,enthaltende“ Sachlage; die Frage, welcher der beiden für das $\mathrm{X}$ eines veridischen bedeutungerfüllenden Aktes besser geeignet ist, lasse ich hier offen. Für beide Kandidaten gelten die folgenden Feststellungen, die zu den im [§48] aufgestellten etwas parallel sind:

(1) Einerseits sind noematische Sätze/Sinne von bedeutungerfüllenden Akten, wie oben erläutert, gewissen durch vorgrammatisch-noematische Kategorien bestimmten vor-grammatischen Gesetzen unterworfen. Es gibt also kein Noema oder noematisches Feld $^{538}$ (von bedeutungerfüllenden Akten), das einen ungrammatischen Satz/Sinn als Moment enthält. (2) Andererseits ist das X (von bedeutungerfüllenden Akten) ein unselbständiges Moment des Noema oder noematischen Feldes. ${ }^{539}$ (1) und (2) zufolge kann das bestimmbare X (von bedeutungerfüllenden Akten) nur in einem Noema oder noematischen Feld, dessen noematischer Satz/Sinn vor-grammatisch wohlgeformt ist, konstituiert werden. In diesem Sinne können wir sagen, dass auch die Konstitution des bestimmbaren $\mathrm{X}$ von bedeutungerfüllenden Akten gewissen durch vorgrammatischnoematische Kategorien bestimmten Gesetzen unterworfen ist. Wir können also die Rede vom Vor-Grammatikalitätsphänomen (bezüglich) des bestimmbaren $X$ von bedeutungerfüllenden Akten einführen:

(Die Grammatikalitätsthese des bestimmbaren X von bedeutungerfüllenden Akten)

Die Konstitution des bestimmbaren $\mathrm{X}$ von bedeutungerfüllenden Akten ist gewissen durch vorgrammatisch-noematische Kategorien bestimmten vorgrammatischen Gesetzen unterworfen, wobei das bestimmbare $\mathrm{X}$ die (Spezifikation des bestimmbaren X des bedeutungerfüllenden Aktes) erfüllt.

Das bezeichnen wir als das Vor-Grammatikalitätsphänomen (bezüglich) des bestimmbaren X von bedeutungerfüllenden Akten.

Da sowohl der noematische Satz/Sinn als auch das bestimmbare X von bedeutungerfüllenden Akten zum Noema oder noematischen Feld angehören, könnte

\footnotetext{
${ }^{537}$ Z.B. sieht Süßbauer die Sachlage als „gegenständliches Korrelat“ der explizierenden Betrachtung. Vgl. Alfons Süßbauer, Intentionalität, Sachverhalt, Noema, S.262, 278.

${ }_{538} \mathrm{Vgl}$. [\$44c] der vorliegenden Arbeit.

539 Aber natürlich kann ein und dasselbe $\mathrm{X}$ in verschiedenen Noemata als ein unselbständiges Moment auftreten. Vgl. [§40] der vorliegenden Arbeit.
} 
man beide Thesen vom Vor-Grammatikalitätsphänomen unter der folgenden These I zusammenfassen und die Rede des noematischen Vor-Grammatikalitätsphänomens von bedeutungerfüllenden Akten einführen:

These I - (Die Grammatikalitätsthese des noematischen Feldes von bedeutungerfüllenden Akten)

Sowohl die noematischen Sätze/Sinne als auch die Konstitution des bestimmbaren $\mathrm{X}$ von bedeutungerfüllenden Akten sind gewissen durch vorgrammatisch-noematische Kategorien bestimmten vor-grammatischen

Gesetzen unterworfen. Das bezeichnen wir als das noematische VorGrammatikalitätsphänomen von bedeutungerfüllenden Akten.

Auf diese Weise haben wir das Vor-Grammatikalitätsphänomen von bedeutungerfüllenden Akten noematisch charakterisiert. In Anlehnung an [\$47] wollen wir noch seine noetische Hinsicht betrachten.

\section{§ 67. Das noetische (Vor-)Grammatikalitätsphänomen von bedeutungerfüllenden Akten}

Im [§47] haben wir, dem Prinzip des noetisch-noematischen Parallelismus folgend, ein paralleles Verhältnis zwischen noematischen und noetischen Komplikationsweisen, strukturelle Isomorphie genannt, im Anwendungsbereich von Bedeutungsintentionen abgeleitet. Meines Erachtens besteht auch im Bereich von bedeutungerfüllenden Akten eine strukturelle Isomorphie zwischen noematischen und noetischen Komplikationsweisen ${ }^{540}$ :

(1) Dem zusammengesetzten noematischen Satz/Sinn eines bedeutungerfüllenden Aktes entspricht auf noetischer Seite der Gesamtakt, und den Sätzen/Sinnen, aus denen der Gesamt-Satz/Sinn des Gesamtaktes besteht, entsprechen auf noetischer Seite Teilakte, aus denen der Gesamtakt besteht. Z.B. hat eine Wahrnehmung, die den Substratgegenstand S nach seinen Bestimmungen p, q, r usw. auf explizierende Weise betrachtet, auf noematischer Seite den entsprechenden Gesamt-Satz/Sinn $\left[[S]_{n^{*}}-[p\right.$

\footnotetext{
${ }^{540}$ Unter dem Gesichtspunkt der Erkenntnisgenesis, wie schon im [§55] erörtert, sind bedeutungerfüllende Akte fundierend für die entsprechenden Bedeutungsintentionen. In diesem Sinne können wir sagen, dass das (Vor-)Grammatikalitätsphänomen von bedeutungerfüllenden Akten grundlegender ist als das von Bedeutungsintentionen.
} 


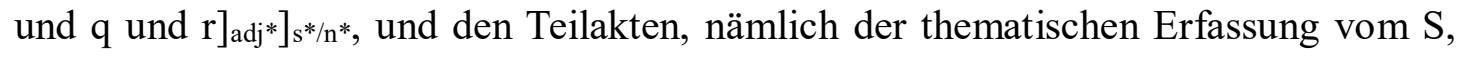
den Sondererfassungen von $\mathrm{p}, \mathrm{q}, \mathrm{q}$ usw. entsprechen jeweils die Teil-Sätze/Sinne von $[\mathrm{S}]_{\mathrm{n} *},[\mathrm{p}]_{\mathrm{adj}} *,[\mathrm{q}]_{\mathrm{adj}},[\mathrm{r}]_{\mathrm{adj}}$ usw.

(2) Die Teil-Sätze/Sinne ([S $]_{n}$, $[\mathrm{p}]_{\mathrm{adj}}$, $[\mathrm{q}]_{\mathrm{adj}}$, $[\mathrm{r}]_{\mathrm{adj}}$ ( usw.) unterstehen gewissen vorgrammatisch-noematischen Kategorien, die bestimmen, auf welche Weise die TeilSätze/Sinne sich zu einem einheitlichen Satz/Sinn von welcher vorgrammatischnoematischen Kategorie zusammenschließen; dadurch wird auf noetischer Seite die Komplikationsweise der entsprechenden Teilakte determiniert: Dem Gesamt-Satz/Sinn

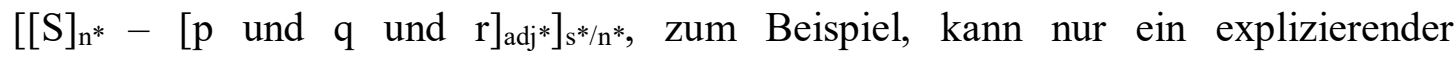
Wahrnehmungsakt entsprechen, der aus den Sonderakten von S, p, q, r durch explikative noetische Deckung besteht.

Daher können wir die Rede des noetischen Vor-Grammatikalitätsphänomens von bedeutungerfüllenden Akten einführen:

These II - (Die Grammatikalitätsthese der Noesis von bedeutungerfüllenden Akten)

Die Noesen von bedeutungerfüllenden Akten sind gewissen durch vorgrammatisch-noematische Kategorien bestimmten vor-grammatischen Gesetzen unterworfen. Das bezeichnen wir als das noetische VorGrammatikalitätsphänomen von bedeutungerfüllenden Akten.

\section{$\S$ 68. Zusammenfassung des Kapitels}

Ziel des Abschnitts war, wie schon erwähnt ${ }^{541}$, die Rede vom Grammatikalitätsphänomen auf die Sphäre von bedeutungerfüllenden Akten systematisch zu erweitern. Dieses Ziel haben wir erreicht: (1) Wir haben erörtert, welche Überlegungen Husserl zu den sogenannten Ursprungsanalysen des Urteils und darunter $\mathrm{zu}$ den Erfahrungsanalysen geführt haben (I.Kapitel); (2) Die Erfahrungsanalysen haben gezeigt, dass schon auf der Erfahrungsstufe des Erkenntnisstrebens vor-syntaktische sowie vor-grammatische Formbildungen

\footnotetext{
${ }^{541} \mathrm{Vgl}$. [§46], [§50] der vorliegenden Arbeit.
} 
stattfinden (II. Kapitel); (3) Aufgrund der dabei herausgearbeiteten vor-grammatischen Formen wird im vorliegenden Kapitel die These I gerechtfertigt eingeführt; dem Prinzip des noetisch-noematischen Parallelismus folgend, haben wir These II abgeleitet.

Terminologisch können wir die Thesen I und II unter dem Gesamttitel (die Grammatikalitätsthese von bedeutungerfüllenden Akten) zusammenfassen und somit die allgemeinere Rede vom noetisch-noematischen Vor-Grammatikalitätsphänomen von bedeutungerfüllenden Akten einführen.

In paralleler Anlehnung an [§49] lässt sich der Zusammenhang der hier aufgestellten Thesen durch die folgende Graphik illustrieren:

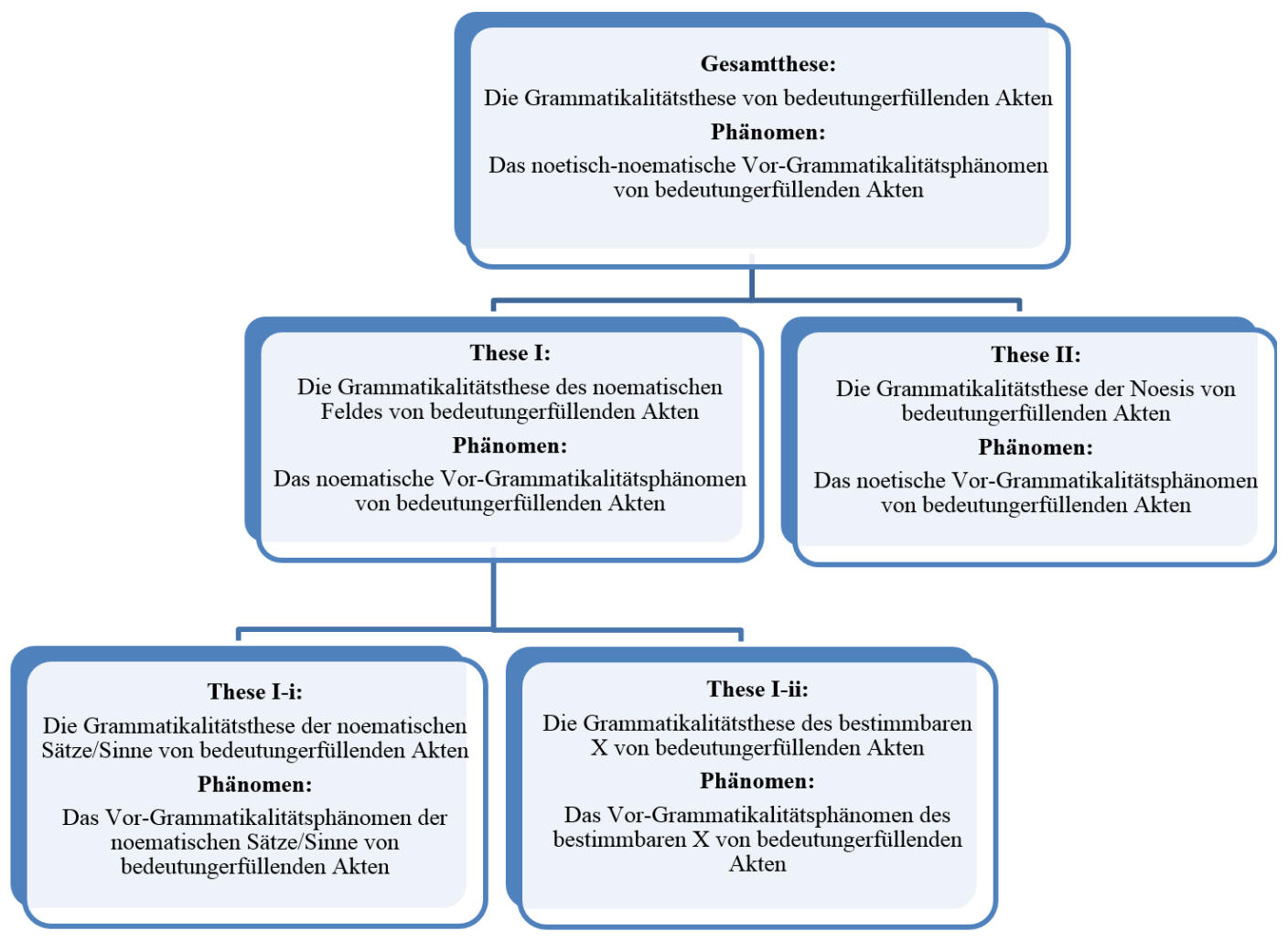

Sorgfältige Leser können leicht bemerken, dass die hier aufgestellten Grammatikalitätsthesen mit den vom [§49] parallelisieren. 


\section{SCHLUSSWORT}

Das im Titel der vorliegenden Arbeit angekündigte Thema - das Grammatikalitätsphänomen - haben wir systematisch studiert: (1) Im I. Abschnitt wurde die Rede des Grammatikaliätsphänomens durch zwei Grammatikalitätsthesen (im [§26]) eingeführt. (2) Im II. Abschnitt sind wir in die transzendentale Phänomenologie eingetreten, um die Grammatikalitätsthesen durch noetischnoematische Konstitutionsanalysen zu reinterpretieren. Dabei haben Wir die im I. Abschnitt eingeführte Rede vom Grammatikalitätsphänomen durch die Gesamtthese die Grammatikalitätsthese von Bedeutungsintentionen - und ihre Teilthesen (im [§49]) re-charakterisiert und erweitert. (3) Im III. Abschnitt sind wir in Husserls Ursprungsanalysen des Urteils und darunter speziell in die Erfahrungsanalysen eingetreten. Besonders durch die Analysen, die die rezeptive Stufe der Erfahrung innerhalb des Erkenntnisstrebens untersuchen, haben wir das (noetisch-noematische) Vor-Grammatikalitätsphänomen von bedeutungerfüllenden Akten herausgearbeitet. (4) Da Bedeutungsintentionen und bedeutungerfüllende Akte zusammen die Klasse von objektivierenden Akten ${ }^{542}$ ausmachen, haben wir also durch die unter (2) und (3) zusammengefassten Untersuchungen das Grammatikalitätsphänomen bezüglich der Klasse von objektivierenden Akten herausgearbeitet. (5) Da die nicht-objektivierenden Akte auf objektivierenden Akten als Grundlage beruhen, ${ }^{543}$ liegt es nahe, die Rede vom Grammatikalitätsphänomen wiederum auf nicht-objektivierende Akte auszuweiten. In diesem Sinne können wir vom Grammatikalitätsphänomen von intentionalen Akten überhaupt mit Recht zu sprechen. ${ }^{544}$

Die vorliegende Arbeit kann eine etwas triviale Meinung phänomenologisch verständlich machen: Die Weise, in der wir über die Welt sprechen, reflektiert (in formaler Hinsicht) die Weise, in der wir die Welt denken und sehen. In diesem Sinne kann die vorliegende Arbeit hoffentlich für den, der sich für den Zusammenhang zwischen Sprechen, Denken und Anschauen interessiert, interessant sein.

\footnotetext{
${ }^{542} \mathrm{Vgl}$. [§5] der vorliegenden Arbeit.

${ }^{543} \mathrm{Vgl}$. [§4] der vorliegenden Arbeit.

${ }^{544}$ Vgl. [\$46] der vorliegenden Arbeit.
} 


\section{APPENDIX \\ Die syntaktische Konnexität von Ajdukiewicz}

Der polnische Logiker Kazimierz Ajdukiewicz ist von Husserls Begriff der Bedeutungskategorie inspiriert und möchte eine Symbolik vorschlagen, durch die die syntaktische Konnexität (= das Wohlgeformtsein) eines gegebenen Ausdrucks „,kalkülmässig definiert und untersucht werden kann “. ${ }^{545}$ Im vorliegenden Appendix möchte ich diese Symbolik kurz darstellen. Dabei zeigt sich auch die interdisziplinäre Leistungsfähigkeit von Husserls Idee der ,reinen Grammatik“: Logiker versuchen, diese programmatische Idee zu verwirklichen und präzisieren.

Husserls Unterscheidung von selbständiger/unselbständiger Bedeutung entsprechend unterscheidet Ajdukiewicz zwischen Grundkategorien und FunktorenKategorien. Grundkategorien sind für Ajdukiewicz Sätze oder Namen, während Funktoren-Kategorien andere Ausdrucksarten sind. In ähnlicher Weise sind bei Husserl selbständige Bedeutungen, wie schon erörtert, Propositionen oder nominale Bedeutungen, während unselbständige Bedeutungen anderen Bedeutungskategorien angehören. ${ }^{546}$ Der Unterschied der beiden Philosophen besteht darin, dass der Term Bedeutungskategorie bei Husserl Arten von Bedeutungen, bei Ajdukiewicz eher Arten von Ausdrücken bezeichnet.

Ajdukiewicz entwirft eine Notation, in der jede gegebene Bedeutungskategorie mit einem Index markiert wird. Indices für die Grundkategorien: die Bedeutungskategorie von Namen ist mit dem Index „n“, und die Bedeutungskategorie von Sätzen mit dem Index ,,s“ gekennzeichnet. Den Ausdrücken, welche zu FunktorenKategorien gehören, will Ajdukiewicz einen Index in Bruchform anschließen: Z.B. wird das Wort „und“, das mit zwei Namen als Argumenten verbunden werden kann (wie in ,Sokrates und Platon“) mit dem Index in Bruchform, $\frac{\mathrm{n}}{\mathrm{n} \cdot \mathrm{n}}$ “ als ein zweistelliger namenbildender Operator markiert. Der Nenner des „Bruchs“ deutet darauf hin, mit welchen Worten von welchen Bedeutungskategorien als Argumenten der Funktor-

\footnotetext{
${ }^{545}$ Kazimierz Ajdukiewicz, ,Die syntaktische Konnexität“, in: Studia Philosophica 1, 1935, S.2.

${ }^{546}$ Vgl. [§8] der vorliegenden Arbeit.
} 
Ausdruck gesättigt werden kann (hier mit zwei Namen), während der Zähler die Bedeutungskategorie des daraus zusammengesetzten Wortes kennzeichnet (hier die Kategorie Name). Weitere Beispiele für Indices von Bedeutungskategorien:

Sub. - „Sokrates“, „Platon“: n

Satz - ,Sokrates läuft“: s

Namenbildende Konjunktion - ,und“‘: $\frac{\mathrm{n}}{\mathrm{n} \cdot \mathrm{n}}$

Satzbildende Konjunktion - ,und“" $\frac{\mathrm{s}}{\mathrm{s} \cdot \mathrm{s}}$

v. - ,laufen“: $\frac{\mathrm{s}}{\mathrm{n}}$; , ,hetzen“: $\frac{\mathrm{s}}{\mathrm{n} \cdot \mathrm{n}}$

adv. - ,schnell“": $\frac{\frac{s}{\mathrm{~s}}}{\frac{\mathrm{s}}{\mathrm{n}}}$

adj.(attributiv) - , ,stark“: $\frac{\mathrm{n}}{\mathrm{n}}$

adj. (prädikativ) - ,ist stark“: $\frac{s}{n}$

usw.

Ajdukiewicz zufolge können wir durch diese Index-Notation kakülmäßig darüber urteilen, ob zwei gegebene Ausdrücke sich zu einem umfassenderen sinnvollen Ausdruck zusammenschließen können. Die Methode ist ähnlich wie die Rechnung von Bruchzahlen. Um z.B. über die syntaktische Konnexität zwischen „Sokrates“ und dem attributiven ,stark“ zu entscheiden, können wir die Indices der beiden wie folgt hinschreiben und ,kalkulieren“:

$$
\begin{array}{cccc}
\text { stark } & \text { Sokrates } & =\text { Name }\left[\text { der }^{547} \text { starke Sokrates }\right] \\
\frac{\mathrm{n}}{\mathrm{n}} & & \mathrm{n} & =\mathrm{n}
\end{array}
$$

Hier lässt sich der Nenner $\left(, n^{\circ}\right)$ des Index von ,,stark“ $\left(,, \frac{n}{n}\right.$ “ $)$ durch den Index von „Sokrates“ („,n“) kürzen, d.i. die Leerstelle des Funktor-Ausdrucks „,stark“ lässt sich durch einen Namen als Argument erfüllen, damit ein umfassenderer Name mit Index ,n“ sich als Produkt ergibt.

Ganz anders verhält es sich bei ,und“ und dem prädikativen Adjektiv ,,ist stark“:

\footnotetext{
${ }^{547}$ Der Einfachheit halber sehen wir hier von dem Index des Artikels hier ab.
} 


$$
\begin{aligned}
& \text { und } \cdot \text { ist stark }=\text { sinnlos } \\
& \frac{\mathrm{n}}{\mathrm{n} \cdot \mathrm{n}} \cdot \frac{\mathrm{s}}{\mathrm{n}}=\text { kein Index }
\end{aligned}
$$

Hier kann weder der Nenner $\left(, n \cdot n^{\prime \prime}\right)$ des ,,und“-Index durch den Index von ,ist $\operatorname{stark}^{\prime}\left(,, \frac{\mathrm{s}}{\mathrm{n}}\right.$ “) noch der Nenner $\left(, \mathrm{n}^{\circ}\right)$ des Index von ,,ist stark“ durch den Index von „,und“ (, $\frac{\mathrm{n}}{\mathrm{n} \cdot \mathrm{n}}$ “) gekürzt werden. Das heißt, die Leerstelle eines der beiden FunktorAusdrucks kann nicht durch den anderen als Argument erfüllt werden. Die Wortverbindung von ,und ist stark“ ist keine sinnvolle Verbindung, oder mit Ajdukiewicz' eignen Worten, sie ist keine syntaktische Konnexität.

Ein anderes Beispiel von „Sokrates läuft schnell““

Sokrates $\cdot$ laufen $\cdot$ schnell $=$ Satz [Sokrates läuft schnell]

$$
\mathrm{n} \quad \cdot\left(\frac{\mathrm{s}}{\mathrm{n}} \cdot \frac{\frac{\mathrm{s}}{\mathrm{s}}}{\frac{\mathrm{s}}{\mathrm{n}}}\right)=\mathrm{n} \cdot \frac{\mathrm{s}}{\mathrm{n}}=\mathrm{s}
$$

Hier lässt sich zuerst der Nenner $\left(,, \frac{s}{n}\right.$ ““ $)$ des Index von „,schnell““ $\left(, \frac{\frac{s}{n}}{\frac{s}{n}}\right.$ “‘ durch den Index von „laufen“ $\left(, \frac{\mathrm{s}}{\mathrm{n}}\right.$ “‘ $)$ kürzen und ergibt den Index von „,schnell laufen“ $\left(,, \frac{\mathrm{s}}{\mathrm{n}}\right.$ “ $)$, der wiederum mit dem Index von „Sokrates“ (,,n“) zum ,,s“ multipliziert wird. ${ }^{548}$ Das Ergebnis ist, wie das Produkt „, “ zeigt, ein sinvoller Satz-Ausdruck.

Es ist hervorzuheben, dass hier nur eine minimale, noch durch andere Regeln $\mathrm{zu}$ ergänzende Notation skizziert wird. Eine ausreichende Entwicklung der von Ajdukiewicz konzipierten Notation ist aber kein Thema der vorliegenden Arbeit. Die Notation ist hier erwähnt als eine Möglichkeit, Husserls programmatische Idee der „reinen Grammatik“ zu verwirklichen und präzisieren.

\footnotetext{
${ }^{548}$ Wir setzen voraus, dass wir in der Notation schon Regeln, die die Reihenfolge der „Multiplikation“ genau so bestimmen, wie es hier der Fall ist, festgesetzt haben.
} 


\section{LITERATUR}

Ajdukiewicz, Kazimierz (1935): Die syntaktische Konnexität, in: Studia Philosophica 1 (1935).

Barber, Michael D. (2008): Holism and Horizon: Husserl and McDowell on Nonconceptual Content, in: Husserl Studies 24 (2008).

Bell, David (1990): Husserl, London/New York 1990.

Benoist, Jocelyn (2008): Grammatik und Intentionalität, in: Mayer (Hrsg.) (2008).

Bernet, R., Kern, I., Marbach, E. (1989): Edmund Husserl. Darstellung seines Denkens, Hamburg 1989.

Bernet, Rudolf (1979): Bedeutung und intentionales Bewußtsein. Husserls Begriff des Bedeutungsphänomens, in: Phänomenologische Untersuchungen 8 (1979).

Bernet, Rudolf (1988): Perception, Categorial Intuition and Truth in Husserl's Sixth 'Logical Investigation', in: Sallis J. C., Moneta G., Taminiaux J. (eds), The Collegium Phaenomenologicum. The First Ten Years, Dordrecht/Boston/London 1988.

Bernet, Rudolf (1990): Husserls Begriff des Noema, in: Ijsseling, Samuel (Hrsg.), Husserl-Ausgabe und Husserl-Forschung, Dordrecht/Boston/London 1990.

Bernet, Rudolf (2008): Intention und Erfüllung, Evidenz und Wahrheit, in: Mayer (Hrsg.) (2008).

Beyer, Christian (1996): Von Bolzano zu Husserl. Eine Untersuchung über den Ursprung der phänomenologischen Bedeutugnslehre, Dordreccht/Boston/London 1996.

Beyer, Chrisitan (1997): Ideen zu einer reinen Phänomenologie der empirischen Bedeutung, in: Phänomenologische Forschungen Neue Folge 2/2 (1997).

Beyer, Christian (2000): Intentionalität Und Referenz. Eine sprachanalytische Studie zu Husserls transzendentaler Phänomenologie, Paderborn 2000.

Beyer, Christian (unveröffentlicht). Analytische Philosophie und husserlsche Phänomenologie.

Beyer, Christian (2001): A Neo-Husserlian Theory of Speaker's Reference, in: Erkenntnis 54 (2001).

Beyer, Christian (2003): Edmund Husserl, in: Stanford Encyclopedia of Philosophy, https://plato.stanford.edu/index.html.

Beyer, Christian (2004): Fiktionale Rede, in: Textor, M. und Siebel, M. (Hrsg.), Semantik und Ontologie. Beiträge zur philosophischen Untersuchung, Frankfurt/Lancaster 2004. 
Beyer, Christian (2013): Husserl's Transcendental Phenomenology Considered in the Light of (Recent) Epistemology, in: Mou, B. und Tieszen, R. (Hrsg.), Constructive Engagement of Analytic and Continental Approaches in Philosophy, Leiden 2013.

Biceaga, Victor (2010): The Concept of Passivity in Husserl's Phenomenology, Dordrecht/Heidelberg/London/New York 2010.

Bonjour, Laurence: Can Empirical Knowledge Have a Foundation?, in: American Philosophical Quarterly 15 (1978).

Brough, John Barnett (1991): Translator's Introduction, in: On the Phenomenology of the Consciousness of Internal Time (1893-1917), Dordrecht/Boston/London 1991.

Carnie, Andrew (2013): Syntax. A Generative Introduction, West Sussex 2013.

Centrone, Stefania (Hrsg.) (2016): Versuche über Husserl, Hamburg 2016.

Donnellan, Keith S. (1966): Reference and Definite Descriptions, in: The Philosophical Review 75 (1966).

Doyon, Maxime (2011): Husserl and McDowell on the Role of Concepts in Perception, in: The New Yearbook for Phenomenology and Phenomenological Philosophy XI (2011).

Dreyfus, Hubert (Hrsg.) (1982): Husserl, Intentionality, and Cognitive Science, Cambridge Mass./London 1982.

Dreyfus, Hubert (1982): Husserl's Perceptual Noema, in: Dreyfus (Hrsg.) (1982).

Drummond, J. und Embree, L. (Hrsg.) (1992): The Phenomenology of the Noema, Dordrecht 1992.

Drummond, John J. (1975): Husserl on the Ways to the Performance of the Reduction, in: Man and World 8 (1975).

Drummond, John J. (1990): Husserlian Intentionality and Non-foundational Realism, Dordrecht 1990.

Drummond, John J. (1992): An Abstract Consideration: De-Ontologizing the Noema, in: Drummond, John J. und Embree, Lester (Hrsg.) (1992).

Drummond, John J. (2008): Historical Dictionary of Husserl's Philosophy, Lanham Md. 2008.

Dummett, Michael (1996): Origions of Analytical Philosophy, London/New Delhi/New York/Sydney 2014.

Edie, J. M. (1977): Husserl's Conception of "The Grammatical" and Contemporary Linguistics, in: Mohanty, J. N. (Hrsg.), Readings on Edmund Husserl's Logical Investigations, The Hague 1977.

Embree, L. et al. (Hrsg.) (1997): Encyclopedia of Phenomenology, Dordrecht 1997. 
Fink, Eugen (1957): Operative Begriffe in Husserls Phänomenologie, in: Zeitschrift für philosophische Forschung 11.

Føllesdal, Dagfinn (1969): Husserl's Notion of Noema, in: The Journal of Philosophy 66 (1969).

Føllesdal, Dagfinn (1982): Husserl's Theory of Perception, in: Dreyfus (Hrsg.) (1982).

Føllesdal, Dagfinn (1990): Noema and Meaning in Husserl, in: Philosophy and Phenomenological Research 50 (1990).

Føllesdal, Dagfinn (1990): Husserl's Idealism, in: Stamm, Marcelo (Hrsg.), Philosophie in synthetischer Hinsicht, Stuttgart 1998.

Frege, Gottlob (1969): Nachgelassene Schriften, hrsg. von Hermes, H., Kambartel, F., and Kaulbach, F., Hamburg 1969.

Frege, Gottlob (1891): Brief an Husserl 24.05.1891, in: Gabriel, Gottfried et al. (Hrsg.), Gottlob Frege. Wissenschaftlicher Briefwechsel, Hamburg 1976.

Frege, Gottlob (1892): Über Sinn und Bedeutung, in: Patzig, Günther (Hrsg.), Gottlob Frege. Funktion, Begriff, Bedeutung, Göttingen 2008.

Frege, Gottlob (1906): Brief an Husserl 09.12.1906, in: Gabriel et al. (Hrsg.) (1976).

Gander, Hans-Helmuth (Hrsg.) (2010): Husserl-Lexikon, Darmstadt 2010.

Gettier, Edmund L. (1963): Is Justified True Belief Knowledge?, Ditzingen 2019.

Gurwitsch, Aron, übersetzt von Fröhlich, W. D. (1975): Das Bewußtseinsfeld, Berlin 1975.

Heffernan, George (1989): Isagoge in die phänomenologische Apophantik, Dordrecht/Boston/London 1989.

Holmes, Richard H. (1975): An Explication of Husserl's Theory of the Noema, in: Research in Phenomenology 5 (1975).

Husserl, Edmund (1939): Erfahrung und Urteil, hrsg. von Landgrebe, Ludwig, Hamburg 1948.

Husserl, Edmund: Cartesianische Meditationen und Pariser Vorträge, hrsg. von Strasser, S., Husserliana I, Den Haag 1950.

Husserl, Edmund: Die Idee der Phänomenologie, hrsg. von Biemel, W., Husserliana II, Den Haag 1950.

Husserl, Edmund: Ideen zu einer reinen Phänomenologie und phänomenologischen Philosophie. Erstes Buch. Allgemeine Einführung in die reine Phänomenologie, hrsg. von Schuhmann, K., Husserliana III/1, Den Haag 1976.

Husserl, Edmund: Die Krisis der europäischen Wissenschaften und die transzendentale Phänomenologie, hrsg. von Biemel, W., Husserliana VI, Den Haag 1954.

Husserl, Edmund: Analysen zur passiven Synthesis, hrsg. von Fleischer, M., 
Husserliana XI. Den Haag 1966.

Husserl, Edmund: Formale und transzendentale Logik, hrsg. von Janssen, P., Husserliana XVII, Den Haag 1974.

Husserl, Edmund: Aufsätze und Rezensionen (1890-1910), hrsg. von Rang, B., Husserliana XXII, Den Haag 1979.

Husserl, Edmund: Logische Untersuchungen. Erster Band. Prolegomena zur reinen Logik, hrsg. von Holenstein, E., Husserliana XVIII, Den Haag 1975.

Husserl, Edmund: Logische Untersuchungen. Zweiter Band. Untersuchungen zur Phänomenologie und Theorie der Erkenntnis, hrsg. von Panzer, U., Husserliana XIX/1 und XIX/2, Den Haag 1984.

Husserl, Edmund: Vorlesungen über Bedeutungslehre. Sommersemester 1908, hrsg. von Panzer, U., Husserliana XXVI, Den Haag 1987.

Kern, Iso (1962): Die drei Wege zur transzendental-phänomenologischen Reduktion in der Philosophie Edmund Husserls, in: Tijdschrift voor Filosofie, 24 (1962).

King, J. C. (2017): The Metaphysics of Propositions, in: Oxford Handbooks Online, https://doi.org/10.1093/oxfordhb/9780199935314.013.26.

Kjosavik, F., Beyer, C. und Fricke, C. (Hrsg.) (2019): Husserl's Phenomenology of Intersubjectivity, London/New York 2019

Kosowski, Łukasz (2010): Noema and Thinkability. An Essay on Husserl's Theory of Intentionality, Heusenstamm 2010.

Kripke, Saul (1977): Speaker's Reference and Semantic Reference, in: Midwest Studies in Philosophy II (1977).

Küng, Guido (1973): Husserl on Pictures and Intentional Objects, in: The Review of Metaphysics 26 (1973).

Künne, Wolfgang (1982a): Indexikalität, Sinn und propositionaler Gehalt, in: Grazer Philosophische Sudien 18 (1982).

Künne, Wolfgang (1982b): Criteria of Abstractness. The Ontologies of Husserl, Frege and Strawson against the Background of Classical Metaphysics, in: Smith (Hrsg.) (1982).

Künne, Wolfgang (1992): Bolzanos blühender Baum. Plädoyer für eine nichtepistemische Wahrheitsauffassung, in: Realismus und Antirealismus, Frankfurt am Main 1992.

Künne, Wolfgang (2013): Intentionalität: Bolzano und Husserl, in: Centrone (Hrsg.) (2016).

Larrabee, Mary Jeanne (1986): The noema in Husserl's phenomenology, in: Husserl 
Studies 3 (1986).

Lohmar, Dieter (1990): Wo lag der Fehler der kategorialen Repräsentation? Zu Sinn und Reichweite einer Selbstkritik Husserls, in: Husserl Studies 7 (1990).

Lohmar, Dieter (1998): Erfahrung und Kategoriales Denken, Dordrecht 1998.

Lohmar, Dieter (2000a): Warum braucht die Logik eine Theorie der Erfahrung, in: Wiegand, O. K. et al. (Hrsg.), Phenomenology on Kant, German Idealism, Hermeneutics and Logic, Dordrecht 2000.

Lohmar, Dieter (2000b): Edmund Husserls „Formale und transzendentale Logik“, Darmstadt 2000.

Lohmar, Dieter (2008): Kategoriale Anschauung, in: Mayer (Hrsg.) (2008).

Lohmar, Dieter (2017): Genetische Phänomenologie, in: Luft, S. und Wehrle, M. (Hrsg.), Husserl-Handbuch. Leben-Werk-Wirkung, Stuttgart 2017.

Lyons, William (1995): Approaches to Intentinoality, Oxford 1995.

Mayer, V. (Hrsg.) (2008): Edmund Husserl. Logische Untersuchungen, Berlin 2008.

Mayer, V. und Erhard, C (2008): Die Bedeutung objektivierender Akte, in: Mayer (Hrsg.) (2008).

Mayer, V. und Erhard, C. (Hrsg.) (2011): Die Aktualität Husserls, Freiburg/München 2011.

McDowell, John (1994): Mind and World, Cambridge Mass./London 1994.

McIntyre, Ronald (1987): Husserl and Frege, in: The Journal of Philosophy 84, (1987).

Mohanty, J.N. (Hrsg.) (1977): Readings on Edmund Husserl's Logical Investigations, The Hague 1977.

Mohanty, J. N. (1976): Edmund Husserl's Theory of Meaning, The Hague 1976.

Mohanty, J. N. (1981): Intentionality and Noema, in: The Journal of Philosophy 78 (1981).

Mohanty, J. N. (1982): Husserl and Frege, Bloomington 1982.

Mooney, Timothy (2010): Understanding and Simple Seeing in Husserl, in: Husserl Studies 26 (2010).

Nenon, Thomas (1997): Two Models of Foundation in the Logical Investigations, in: Hopkins, B. C. (Hrsg.), Husserl in Contemporary Context. Prospects and Projects for Phenomenology, Dordrecht 1997.

Patzig, Günther (2001): Kritische Bemerkungen zu Husserls Thesen über das Verhältnis von Wahrheit und Evidenz, in: Cramer, K. und Beyer, C. (Hrsg.), Edmund Husserl 1859-2009. Beiträge aus Anlass der 150. Wiederkehr des Geburtstages des Philosophen, Berlin/Boston 2001.

Putnam, Hilary (1975): The Meaning of ,meaning", in: Mind, Language and Reality, 
Cambridge 1975.

Putnam, Hilary (1987): The Many Faces of Realism, Chicago 1987.

Reinach, Aldolf (1911): Zur Theorie des negativen Urteils, in: Münchener Philosophische Abhandlungen. Festschrift für Theodor Lipps, Leipzig 1911.

Searle, John (1982): What is an Intentional State? , in:_Dreyfus (Hrsg.) (1982).

Searle, John (1983): Intentionality, Cambridge 1983.

Smith B. und Smith D. W. (Hrsg.) (1995): The Cambridge Companion to Husserl, Cambridge 1995.

Smith, A.D. (2003): Husserl and the Cartesian Meditations, London/New York 2003.

Smith, A.D. (2006): Husserl and Externalism, in: Synthese 160 (2008).

Smith, Barry (Hrsg.) (1982): Parts and Moments, München/Wien 1982.

Smith, Barry (1982): Introduction to Adolf Reinach. On the Theory of the Negative Judgement, in: Smith (Hrsg.) (1982).

Smith, D. W. und McIntyre, R. (1982): Husserl and Intentionality, Dordrecht/Boston/Lancaster 1982.

Smith, D. W. und McIntyre, R. (1975): Husserl's Identification of Meaning and Noema, in: The Monist 59 (1975).

Smith, D.W. (1979): The Case of the Exploding Perception, in: Synthese 41(2) (1979).

Smith, D.W. (2007): Husserl, London/New York 2007.

Sokolowski, Robert (1970): The Formation of Husserl's Concept of Constitution, The Hague 1970.

Sokolowski, Robert (1974): Husserlian Meditations. How Words Present Things, Evanston 1974.

Sokolowski, Robert (1984): Intentional Analysis and the Noema, in: Dialectica 38 (1984).

Sokolowski, Robert (1987): Husserl and Frege, in: The Journal of Philosophy 84 (1987).

Sokolowski, Robert (2000): Introduction to Phenomenology, Cambridge 2000.

Staiti, Andrea (Hrsg.) (2015): Commentary on Husserl's “Ideas I”, Berlin 2015.

Steinbock A. J. (1998): Husserl's Static and Genetic Phenomenology: Traslator's Introduction to two Essays, in: Continental Philosophy Review 31 (1998).

Ströker, Elisabeth (1984): Intentionalität und Konstitution. Wandlungen des Intentionlitätskonzepts in der Philosophie Husserls, in: Dialectica, 38 (1984).

Ströker, Elisabeth (1987): Husserls transzendentale Phänomenologie, Frankfurt am Main 1987.

Süßbauer, Alfons (1995): Intentionalität, Sachverhalt, Noema, Freiburg/München 1995. 
Tugendhat, Ernst (1965/66): Der Wahrheitsbegriff bei Husserl und Heidegger, Berlin 1970.

Tugendhat, Ernst (1976): Vorlesungen zur Einführung in die sprach-analytische Philosophie, Frankfurt am Main 1976.

Twardowski, Kasimir (1894): Zur Lehre vom Inhalt und Gegenstand der Vorstellungen, München/Wien 1982.

Van Maziik, Corijn (2014): Kant, Husserl, Mcdowell: The Non-conceptual in Experience, in: Diametros 41 (2014).

Welton, Donn (1987): Frege and Husserl on Sense, in: The Journal of Philosophy 84 (1987).

Welton, Donn (2000): The Other Husserl, Bloomington/Indianapolis 2000.

Williford, K. (2013): Husserl's Hyletic Data and Phenomenal Consciousness, in: Phenomenology and the Cognitive Sciences 12(3) (2013).

Zahavi, Dan (2003): Husserls Phenomenology, Stanford 2003.

Zahavi, Dan (2017): Husserl's Legacy, Oxford 2017. 


\section{Rechtsverbindliche Erklärung}

Hiermit versichere ich an Eides statt, dass ich die eingereichte Dissertation mit dem Titel „Das Grammatikalitätsphänomen in Husserls Phänomenologie“ selbständig und ohne unerlaubte Hilfe verfasst habe. Anderer als der von mir angegebenen Hilfsmittel und Schriften habe ich mich nicht bedient. Alle wörtlich oder sinngemäß den Schriften anderer Autorinnen oder Autoren entnommenen Stellen habe ich kenntlich gamacht. Die Abhandlung ist noch nicht veröffentlicht worden und noch nicht Gegenstand eines Promotionsverfahrens gewesen.

Ich versichere zudem, dass die digitale Version dieser Dissertation mit der schriftlichen wissenschaftlichen Abhandlung übereinstimmt.

Göttingen, 06. September 2020

Jiahao Wu 